\title{
Hadronization and Solvable Models of Renormdynamics of QCD
}

\author{
Nugzar Makhaldiani* \\ JINR \\ E-mail: mnvdjinr.ru
}

Independently radiating valence quarks and corresponding negative binomial distribution presents phenomenologically preferable mechanism of hadronization in multiparticle production processes. Main properties of the renormdynamics, corresponding motion equations and their solutions are considered on the examples of QCD and other field theory models.

Talk presented at the XXII International Baldin Seminar on High Energy Physics Problems "Relativistic Nuclear Physics and Quantum Chromodynamics"

September 15 to 20, 2014 in Dubna, Russia.

\footnotetext{
* Speaker.
} 
Wife raised the small child

To witness his last breath:

"Look son, and lern

How men go to their rest

And think on vengeance

From your mother's breast!"

(Lermontov, Ballad)

Mikhail Lermontov was born on October 15 (October 3 in old style) in 1814 in Moscow.

In 1837 the poet was exiled to the Caucasus for his poem "Poet's Death".

Lermontov's poems "Demon" "Mtsyri" his great novel "A Hero of Our Time" and

his play "Masquerade" are masterpieces of Russian literature.

On July 15th, 1841 the poet was killed. He was not even 27 at that time.

\section{New Physics}

We say that we find New Physics when either we find a phenomenon which is forbidden by SM in principal - this is the qualitative level of New physics - or we find significant deviation between precision calculations in SM of an observable quantity and corresponding experimental value.

In 1900, the British physicist Lord Kelvin is said to have pronounced: "There is nothing new to be discovered in physics now. All that remains is more and more precise measurement." Within three decades, quantum mechanics and Einstein's theory of relativity had revolutionized the field. Today, no physicist would dare assert that our physical knowledge of the universe is near completion. To the contrary, each new discovery seems to unlock a Pandora's box of even bigger, even deeper physics questions.

\section{Renormdynamics}

Renordynamics unifies

Different renormgroups in one society.

Quantum field theory (QFT) and Fractal calculus (FC) provide Universal language of fundamental physics (see e.g. [Makhaldiani, 201]). In QFT existence of a given theory means, that we can control its behavior at some scales (short or large distances) by renormalization theory [Collins, 1984]. If the theory exists, than we want to solve it, which means to determine what happens on other (large or short) scales. This is the problem (and content) of Renormdynamics. The result of the Renormdynamics, the solution of its discrete or continual motion equations, is the effective QFT on a given scale (different from the initial one).

We can invent scale variable $\lambda$ and consider QFT on $D+1+1$ dimensional space-time-scale. For the scale variable $\lambda \in(0,1]$ it is natural to consider $q$-discretization, $0<q<1, \lambda_{n}=q^{n}, n=$ $0,1,2, \ldots$ and $p$ - adic, nonarchimedian metric, with $q^{-1}=p$ - prime integer number. 
The field variable $\varphi(x, t, \lambda)$ is complex function of the real, $\mathrm{x}, \mathrm{t}$, and $\mathrm{p}$ - adic, $\lambda$, variables. The solution of the UV renormdynamic problem means, to find evolution from finite to small scales with respect to the scale time $\tau=\ln \lambda / \lambda_{0} \in(-\infty, 0)$. Solution of the IR renormdynamic problem means to find evolution from finite to the large scales, $\tau=\ln \lambda / \lambda_{0} \in(0, \infty)$. This evolution is determined by Renormdynamic motion equations with respect to the scale-time. In multiplicatively renormalization case $\varphi(x, t, \lambda)=Z\left(\lambda, \lambda_{0}\right) \varphi\left(x, t, \lambda_{0}\right)$, where $Z\left(\lambda, \lambda_{0}\right)$ is real function of $\mathrm{p}$-adic variable $\lambda$. As a concrete model, we take a relativistic scalar field model with lagrangian

$$
L=\frac{1}{2} \partial_{\mu} \varphi \partial^{\mu} \varphi-\frac{m^{2}}{2} \varphi^{2}-\frac{g}{n} \varphi^{n}, \mu=0,1, \ldots, D-1
$$

The mass dimension of the coupling constant is

$$
[g]=d_{g}=D-n \frac{D-2}{2}=D+n-\frac{n D}{2} .
$$

In the case

$$
\begin{aligned}
& n=\frac{2 D}{D-2}=2+\frac{4}{D-2}=2+\varepsilon(D) \\
& D=\frac{2 n}{n-2}=2+\frac{4}{n-2}=2+\varepsilon(n)
\end{aligned}
$$

the coupling constant $\mathrm{g}$ is dimensionless, and the model is renormalizable.

We take the euklidean form of the QFT which unifies quantum and statistical physics problems. In the case of the QFT, we can return (in)to minkowsky space by transformation: $p_{D}=i p_{0}, x_{D}=$ $-i x_{0}$.

The main objects of the theory are Green functions - correlation functions - correlators,

$$
\begin{aligned}
& G_{m}\left(x_{1}, x_{2}, \ldots, x_{m}\right)=<\varphi\left(x_{1}\right) \varphi\left(x_{2}\right) \ldots \varphi\left(x_{m}\right)> \\
& =Z_{0}^{-1} \int d \varphi(x) \varphi\left(x_{1}\right) \varphi\left(x_{2}\right) \ldots \varphi\left(x_{m}\right) e^{-S(\varphi)}
\end{aligned}
$$

where $d \varphi$ is an invariant measure,

$$
d(\varphi+a)=d \varphi
$$

For gaussian actions,

$$
S=S_{2}=\frac{1}{2} \int d x d y \varphi(x) A(x, y) \varphi(y)=\varphi \cdot A \cdot \varphi
$$

the QFT is solvable,

$$
\begin{aligned}
& G_{m}\left(x_{1}, \ldots, x_{m}\right)=\left.\frac{\delta^{m}}{\delta J\left(x_{1}\right) \ldots J\left(x_{m}\right)} \ln Z_{J}\right|_{J=0}, \\
& Z_{J}=\int d \varphi e^{-S_{2}+J \cdot \varphi}=\exp \left(\frac{1}{2} \int d x d y J(x) A^{-1}(x, y) J(y)\right) \\
& =\exp \left(\frac{1}{2} J \cdot A^{-1} \cdot J\right)
\end{aligned}
$$

This solution is based on the solution of the linear motion equations with sources

$$
\int A(x, y) \varphi(y) d y=J(x)
$$

Nontrivial problem is to calculate correlators for non gaussian QFT. This case, in the perturbative theory or beyond, the canonical-classical dimensions of fields of particles and constants, receive (non)perturbative 'anomalous' corrections and become fractal objects. 


\subsection{Effective action}

Generating functional for connected correlators is

$$
F(J)=\ln Z_{J}, \frac{\delta F(J)}{\delta J(x)}=\frac{1}{Z_{J}} \frac{\delta Z_{J}}{\delta J(x)} \equiv<\varphi(x)>_{J} \equiv \varphi(x)-
$$

is observable value of the field, generated by source $J$. We have

$$
\left.\frac{\delta}{\delta J}(F(J)-J \cdot \varphi)\right|_{\varphi=c o n s t}=0,
$$

so

$$
\begin{aligned}
& J \cdot \varphi-F(J)=S_{q}(\varphi)=S(\varphi)+R(\varphi) \\
& =\sum_{n \geq 1} \frac{1}{n !} \int d x_{1} d x_{2} \ldots d x_{n} \Gamma_{n}\left(x_{1}, x_{2}, \ldots, x_{n}\right) \varphi\left(x_{1}\right) \varphi\left(x_{2}\right) \ldots \varphi\left(x_{n}\right), \\
& \frac{\delta S_{q}}{\delta \varphi(x)}=J(x) ; \frac{\delta^{2} S_{q}}{\delta \varphi\left(x_{1}\right) \delta \varphi\left(x_{2}\right)}=\frac{\delta J\left(x_{2}\right)}{\delta \varphi\left(x_{1}\right)}=\frac{\delta J\left(x_{1}\right)}{\delta \varphi\left(x_{2}\right)}=\Gamma_{2}\left(x_{1}, x_{2}\right)
\end{aligned}
$$

$R(\phi)$ - is quantum corrections to the classical action.

The connected part of the two point correlator - propagator, is

$$
\begin{aligned}
& <\varphi\left(x_{1}\right) \varphi\left(x_{2}\right)>_{c}=<\varphi\left(x_{1}\right) \varphi\left(x_{2}\right)>-<\varphi\left(x_{1}\right)><\varphi\left(x_{2}\right)> \\
& =\frac{1}{Z(J)} \frac{\delta^{2} Z(J)}{\delta J\left(x_{1}\right) \delta J\left(x_{2}\right)}-\frac{1}{Z(J)} \frac{\delta Z(J)}{\delta J\left(x_{1}\right)} \frac{1}{Z(J)} \frac{\delta Z(J)}{\delta J\left(x_{2}\right)}=\Gamma_{2}\left(x_{1}, x_{2}\right)
\end{aligned}
$$

\subsection{One loop approximation and zeta-function regularization}

When the classical motion equation has a solution $\varphi_{c}$, we can consider the quantum corrections in the harmonic or one-loop approximation

$$
S_{q}\left(\varphi_{c}+\chi\right)=S\left(\varphi_{c}\right)+\chi S^{(2)}\left(\varphi_{c}\right) \chi+\ldots
$$

In the case of the action

$$
\begin{aligned}
& S(\varphi)=\int d^{D} x\left(\frac{1}{2} \varphi\left(\Delta+m^{2}\right) \varphi+U(\varphi)\right) \\
& \chi S^{(2)}\left(\varphi_{c}\right) \chi=\int d x^{D}\left(\frac{1}{2} \chi\left(\Delta+m^{2}+U^{\prime \prime}\left(\varphi_{c}\right)\right) \chi\right), \\
& F\left(\varphi_{c}\right)=-S\left(\varphi_{c}\right)+\frac{1}{2} \operatorname{tr} \ln A, A=\left(\Delta+m^{2}+U^{\prime \prime}\left(\varphi_{c}\right)\right), \\
& \operatorname{det} A=\left(\prod a_{n}=e^{\Sigma_{n} \ln a_{n}}\right)=e^{t r \ln A}
\end{aligned}
$$

In the zeta-function regularization format,

$$
\begin{aligned}
& \zeta_{A}(s)=\sum_{n} \lambda_{n}^{-s}=t r A^{-s} \\
& \operatorname{tr} \ln A=-\zeta_{A}^{\prime}(0)
\end{aligned}
$$




\subsection{Convergence of Perturbation Theory Series}

Perturbation theory series (PTS) have the following qualitative form

$$
\begin{aligned}
& f(g)=f_{0}+f_{1} g+\ldots+f_{n} g^{n}+\ldots, f_{n}=n ! P(n) \\
& f(x)=\sum_{n \geq 0} P(n) n ! x^{n}=P(\delta) \Gamma(1+\delta) \frac{1}{1-x}, \delta=x \frac{d}{d x}
\end{aligned}
$$

So, we reduce previous series to the standard geometric progression series. This series is convergent for $|x|<1$ or for $|x|_{p}=p^{-k}<1, x=p^{k} a / b, k \geq 1$. With proper nomalization of the expansion parametre, the coefficients of the series are rational numbers and if experimental data indicates for some prime value for $g$, e.g. in QED

$$
g=\frac{e^{2}}{4 \pi}=\frac{1}{137.0 \ldots}
$$

then we can take corresponding prime number and consider p-adic convergence of the series. In the case of QED, we have

$$
f(g)=\sum f_{n} p^{-n}, f_{n}=n ! P(n), p=137,|f|_{p} \leq \sum\left|f_{n}\right|_{p} p^{n}
$$

In the Yukawa theory of strong interactions (see e.g. [Bogoliubov, Shirkov, 1959]), we take $g=13$,

$$
\begin{aligned}
& f(g)=\sum f_{n} p^{n}, f_{n}=n ! P(n), p=13, \\
& |f|_{p} \leq \sum\left|f_{n}\right|_{p} p^{-n}<\frac{1}{1-p^{-1}}
\end{aligned}
$$

So, the series is convergent. If the limit is rational number, we consider it as an observable value of the corresponding physical quantity.

Note also, that the inverse coupling expansions, e.g. in lattice(gauge) theories,

$$
f(\beta)=\sum r_{n} \beta^{n},
$$

are also p-adically convergent for $\beta=p^{k}$. We can take the following scenery. We fix coupling constants and masses, e.g in QED or QCD, in low order perturbative expansions. Than put the models on lattice and calculate observable quantities as inverse coupling expansions, e.g.

$$
\begin{aligned}
& f(\alpha)=\sum r_{n} \alpha^{-n}, \\
& \alpha_{Q E D}(0)=1 / 137 ; \alpha_{Q C D}\left(m_{Z}\right)=0.11 \ldots=1 / 3^{2}
\end{aligned}
$$

In the period between 1935 and 1965, many attempts at formulating a theory of the strong interaction based on elementary fields for baryons and mesons were made. In particular, uncountable $\mathrm{PhD}$ theses were written, based on local interactions of the Yukawa type, using perturbation theory to analyze them, despite the fact that the coupling constants invariably turned out to be numerically large. Absolutely nothing worked even half way.

In MSSM (see [Kazakov, 2004]) coupling constants unifies at $\alpha_{u}^{-1}=26.3 \pm 1.9 \pm 1$. So,

$$
23.4<\alpha_{u}^{-1}<29.2
$$


Question: how many primes are in this interval?

$$
24,25,26,27,28,29
$$

Only one!

Proposal: take the value $\alpha_{u}^{-1}=29.0 \ldots$ which will be two orders of magnitude more precise prediction and find the consequences for the $S M$ scale observables.

Let us make more explicit the formal representation of ([2.16)

$$
\begin{aligned}
& f(x)=\sum_{n \geq 0} P(n) n ! x^{n}=P(\delta) \Gamma(1+\delta) \frac{1}{1-x}, \\
& =P(\delta) \int_{0}^{\infty} d t e^{-t} t^{\delta} \frac{1}{1-x}=P(\delta) \int_{0}^{\infty} d t \frac{e^{-t}}{1+(-x) t}, \delta=x \frac{d}{d x}
\end{aligned}
$$

This integral is well defined for negative values of $x$. The Mathematica answer for the corresponding integral is

$$
I(x)=\int_{0}^{\infty} d t \frac{e^{-t}}{1+x t}=e^{1 / x} \Gamma(0,1 / x) / x, \operatorname{Im}(x) \neq 0, \operatorname{Re}(x) \geq 0,
$$

where $\Gamma(a, z)$ is the incomplete gamma function

$$
\Gamma(a, z)=\int_{z}^{\infty} d t t^{a-1} e^{-t}
$$

For $x=0.001, I(x)=0.999$

Note that, $y=I(x)$ is the formal solution of the following differential equation

$$
x^{2} y^{\prime}+(x+1) y=1, y=\sum_{n \geq 0}(-1)^{n} n ! x^{n}
$$

Indeed,

$$
\sum_{n \geq 0}(-1)^{n} n ! n x^{n+1}+\sum_{n \geq 0}(-1)^{n} n ! x^{n+1}+1-\sum_{n \geq 0}(-1)^{n}(n+1) ! x^{n+1}=1
$$

There are qualitative difference between positive and negative values of $x$ for the integral and series representations of $I(x)$. In the differential equation on signs of $x$ qualitatively depends only coefficient $(x+1)$, which has zero for negative $x$.

\section{The Goldberger-Treiman Relation and the Pion-Nucleon Coupling Constant}

The Goldberger-Treiman relation (GTR) [Goldberger, Treiman, 1958] plays an important role in theoretical hadronic and nuclear physics. GTR relates the Meson-Nucleon coupling constants to the axial-vector coupling constant in $\beta$-decay:

$$
g_{\pi N} f_{\pi}=g_{A} m_{N}
$$

where $m_{N}$ is the nucleon mass, $g_{A}$ is the axial-vector coupling constant in nucleon $\beta$-decay at vanishing momentum transfer, $f_{\pi}$ is the $\pi$ decay constant and $g_{\pi N}$ is the $\pi-N$ coupling constant. 
Since the days when the Goldberger-Treiman relation was discovered, the value of $g_{A}$ has increased considerably. Also, $f_{\pi}$ decreased a little, on account of radiative corrections. The main source of uncertainty is $g_{\pi N}$.

If we take

$$
\alpha_{\pi N}=\frac{g_{\pi N}^{2}}{4 \pi}=13 \Rightarrow g_{\pi N}=12.78
$$

the proton mass $m_{p}=938 \mathrm{MeV}$ and $f_{\pi}=93 \mathrm{MeV}$, from (B.]), we find

$$
g_{A}=\frac{f_{\pi} g_{\pi N}}{m_{N}}=\frac{93 \times \sqrt{52 \pi}}{938}=1.2672
$$

which is in agreement with contemporary experimental value $g_{A}=1.2695(29)$

In an old version of the unified theory [Heisenberg 1966], for the $\alpha_{\pi N}$ the following value were found

$$
\alpha_{\pi N}=4 \pi\left(1-\frac{m_{\pi}^{2}}{3 m_{p}^{2}}\right)=12.5
$$

Determination of $g_{\pi N}$ from $N N, N \bar{N}$ and $\pi N$ data by the Nijmegen group [Rentmeester et al, 1999] gave the following value

$$
\begin{aligned}
& g_{\pi N}=13.05 \pm .08, \Delta=1-\frac{g_{A} m_{N}}{g_{\pi N} f_{\pi}}=.014 \pm .009, \\
& 13.39<\alpha_{\pi N}<13.72
\end{aligned}
$$

This value is consistent with assumption $g_{\pi N}=13 \Rightarrow \alpha_{\pi N}=13.45$

Due to the smallness of the $\mathrm{u}$ and $\mathrm{d}$ quark masses, $\Delta$ is necessarily very small, and its determination requires a very precise knowledge of the $g_{\pi N}$ coupling $\left(g_{A}\right.$ and $f_{\pi}$ are already known to enough precision, leaving most of the uncertainty in the determination of $\Delta$ to the uncertainty in $\left.g_{\pi N}\right)$.

In the dimensionless ratio, $f_{\pi} / m_{p}=93 / 938$, the numerate $93=3 \times 31=N_{c} / \alpha_{l u}, \alpha_{l u}^{-1}=$ $31, N_{c}=3$

\subsection{Vector meson dominance}

Following the pion, the rho is the most prominent meson. Vector mesons play an important role when considering the interaction of hadrons with electromagnetic fields. In the vector meson dominance model the hadrons couple to photons exclusively through intermediate vector mesons. The equality of the $\rho$ meson self-coupling $g$ and the coupling to nucleons $g_{\rho N}$ and pions $g_{\rho \pi}$, the universality of the $\rho$ meson coupling, plays an important role in vector meson dominance [Sakurai, 1969] and is a consequence of the existence of a consistent EFT with $\rho$ mesons, pions, and nucleons. Indeed, one can rewrite the Lagrangian of [Weinberg, 1968] in terms of renormalized fields and couplings, thereby introducing the basic Lagrangian

$$
\begin{aligned}
L_{R}= & \bar{N}(i \gamma \partial-M) N-\frac{1}{2} \pi\left(\partial^{2}+m^{2}\right) \pi-\frac{1}{4}\left(\partial_{\mu} \rho_{v}^{a}-\partial_{v} \rho_{\mu}^{a}\right)^{2}+\frac{1}{2} M_{\rho}^{2} \rho^{2} \\
& +g \bar{N} \gamma^{u} t_{a} N \rho_{\mu}^{a}+g_{\pi \rho} \varepsilon^{a b c} \pi^{a} \partial^{\mu} \pi^{b} \rho_{\mu}^{c}-g\left(\rho_{\mu} \times \rho_{v}\right) \cdot \partial^{\mu} \rho^{v}-\frac{g^{2}}{4}\left(\rho_{\mu} \times \rho_{v}\right)^{2}
\end{aligned}
$$

Requiring that the results are UV finite introduces relations between the couplings of the theory [Djukanovic et al, 2004], $g_{\pi \rho}=g$. The coupling $g$ is directly related to the width of the $\rho$ meson. 


\subsection{Pion-nucleon coupling constant}

In the previous $\pi \rho N$ model of pion-nucleon interaction [Di Giacomo, Paffuti, Rossi, 1992]

$$
L_{\pi N}=g\left(\bar{N} \gamma^{\mu} t_{a} N+\varepsilon^{a b c} \pi^{b} \partial^{\mu} \pi^{c}\right) \rho_{\mu}^{a}
$$

pion interacts with nucleon through the exchange of the vector meson $\rho\left(m_{\rho}=750 \mathrm{MeV}, \mathrm{T}=1\right)$, the amplitude of $\rho^{0} \rightarrow \pi^{+} \pi^{-}$decay is

$$
M=g \varepsilon^{\mu}\left(k_{\pi^{-}}-k_{\pi^{+}}\right) \mu,
$$

the decay width is

$$
\Gamma=\frac{1}{2 m_{\rho}}|M|^{2}\left(1-\frac{4 m_{\pi}^{2}}{m_{\rho}^{2}}\right)^{\frac{1}{2}} \frac{1}{8 \pi}=\frac{g^{2}}{48 \pi} m_{\rho}\left(1-\frac{4 m_{\pi}^{2}}{m_{\rho}^{2}}\right)^{\frac{3}{2}}
$$

and for fine structure coupling constant we have

$$
\alpha_{\pi \rho N}=\frac{g^{2}}{4 \pi}=\frac{\Gamma}{m_{\rho}} \frac{12}{\left(1-\frac{4 m_{\pi}^{2}}{m_{\rho}^{2}}\right)^{\frac{3}{2}}}=\frac{12 .}{5\left(1-\frac{4 \times 14^{2}}{75^{2}}\right)^{\frac{3}{2}}}=3.006=3.0 .
$$

for $\Gamma=\Gamma_{\rho \pi \pi}=150 \mathrm{MeV}, m_{\pi}=140 \mathrm{MeV}, m_{\rho}=750 \mathrm{MeV}$. So, in this strong coupling model the expansion parameter is a prime number, $\alpha_{g}=3$.

\subsection{Kawarabayashi-Suzuki- Riazuddin-Fayyazuddin relation and rho meson mass}

The KSRF relation [Kawarabayashi, Suzuki, 1966], [Riazuddin, Fayyazuddin, 1966],

$$
g_{\pi \rho}=\frac{M_{\rho}^{2}}{2 g f_{\pi}^{2}},
$$

when $g=g_{\pi \rho}$ reduce to the following

$$
\alpha_{\pi \rho N}=\frac{M_{\rho}^{2}}{8 \pi f_{\pi}^{2}}=3
$$

Than

$$
M_{\rho}=2 \sqrt{6 \pi} f_{\pi}=799 \mathrm{MeV}, f_{\pi}=92 \mathrm{MeV}
$$

If we take the experimental value of the mass, we obtain

$$
M_{\rho}=775.5 \pm 0.4, f_{\pi}=\frac{M_{\rho}}{2 \sqrt{6 \pi}}=89.4
$$

If we take experimental values of $M_{\rho}$ and $f_{\pi}$, for $\alpha_{\pi \rho N}$ we find

$$
\alpha_{\pi \rho N}=\frac{M_{\rho}^{2}}{8 \pi f_{\pi}^{2}}=2.83
$$




\section{Neutral pion to two photon decay}

After integrating out all heavy and trapped particles, we would expect the effective Lagrangian for

$$
\pi^{0} \rightarrow 2 \gamma
$$

to be given by the unique gauge and Lorentz-invariant term with no more than two derivatives:

$$
L_{\pi \gamma \gamma}=g \pi^{0} \varepsilon^{\mu \nu \rho \sigma} F_{\mu \nu} F_{\rho \sigma}
$$

where $g$ is an unknown constant with the mass dimension $m^{-1}$.

The rate for $\pi^{0} \rightarrow 2 \gamma$ is

$$
\Gamma\left(\pi^{0} \rightarrow 2 \gamma\right)=\frac{g^{2} m_{\pi}^{3}}{\pi}
$$

One might naively expect $g$ to be of order

$$
g=\frac{a^{2}}{F_{\pi}}, a=\frac{e}{4 \pi}
$$

where $F_{\pi}=190 \mathrm{MeV}$ is used as a typical strong interaction mass scale.

In 1949, using the pre-QCD theory of pions and nuclons with interaction lagrangian

$$
L_{\pi N N}=i g_{\pi N} \pi^{a} \bar{N} 2 t^{a} \gamma_{5} N
$$

Steinberger calculated the contribution to $g$ from triangle graphs with a single proton loop

$$
g=\frac{e^{2} g_{\pi N}}{32 \pi^{2} m_{N}}=a^{2} \frac{g_{\pi N}}{2 m_{N}}, a=\frac{e}{4 \pi} .
$$

From Goldberger-Treiman relation we have

$$
\frac{g_{\pi N}}{2 m_{N}}=\frac{g_{A}}{2 f_{\pi}}
$$

so,

$$
g=\frac{a^{2}}{F_{\pi}} g_{A}, g_{A}=1.25, F_{\pi}=2 f_{\pi}=184 M e V
$$

Using

$$
\begin{gathered}
g=\frac{a^{2}}{F_{\pi}}, a=\frac{e}{4 \pi}, F_{\pi}=190 \mathrm{MeV},\left(f_{\pi}=190 / 2=95\right) \\
\Gamma\left(\pi^{0} \rightarrow 2 \gamma\right)=\frac{g^{2} m_{\pi}^{3}}{\pi}=\frac{a^{4} m_{\pi}^{3}}{\pi F_{\pi}^{2}}=\frac{\alpha^{2} m_{\pi}^{3}}{16 \pi^{3} F_{\pi}^{2}}=1.0 \times 10^{16} \mathrm{~s}^{-1}
\end{gathered}
$$

The observed rate is

$$
\Gamma\left(\pi^{0} \rightarrow 2 \gamma\right)_{\exp }=(1.19 \pm 0.08) \times 10^{16} s^{-1},
$$

which is in good agreement with the estimation. The observed rate in $\mathrm{KeV}^{\prime} \mathrm{s}$ is

$$
\Gamma\left(\pi^{0} \rightarrow 2 \gamma\right)_{\text {exp }}=(7.7 \pm 0.4) \times 10^{-3} \mathrm{KeV},
$$




\section{Renormdynamics of QCD}

It is sixty years since Yang and Mills (1954) performed their pioneering work on gauge theories. In the standard model of particle physics, the strong force is described by the theory of quantum chromodynamics (QCD). At ordinary temperatures or densities this force just confines the quarks into composite particles (hadrons) of size around $10^{-15} \mathrm{~m}=1$ femtometer $=1 \mathrm{fm}$ (corresponding to the QCD energy scale $\Lambda_{Q C D}=200 \mathrm{MeV}$ ) and its effects are not noticeable at longer distances. However, when the temperature reaches the QCD energy scale (T of order $10^{12}$ kelvins) or the density rises to the point where the average inter-quark separation is less than $1 \mathrm{fm}$ (quark chemical potential $\mu$ around $400 \mathrm{MeV}$ ), the hadrons are melted into their constituent quarks, and the strong interaction becomes the dominant feature of the physics. Such phases are called quark matter or QCD matter or Gluquar. The strength of the color force makes the properties of quark matter unlike gas or plasma, instead leading to a state of matter more reminiscent of a liquid. At high densities, quark matter is a Fermi liquid, but is predicted to exhibit color superconductivity at high densities and temperatures below $10^{12} \mathrm{~K}$.

QCD is the theory of the strong interactions with, as only inputs, one mass parameter for each quark species and the value of the QCD coupling constant at some energy or momentum scale in some renormalization scheme. This last free parameter of the theory can be fixed by $\Lambda_{Q C D}$, the energy scale used as the typical boundary condition for the integration of the Renormdynamic (RD) equation for the strong coupling constant. This is the parameter which expresses the scale of strong interactions, the only parameter in the limit of massless quarks. While the evolution of the coupling with the momentum scale is determined by the quantum corrections induced by the renormalization of the bare coupling and can be computed in perturbation theory, the strength itself of the interaction, given at any scale by the value of the renormalized coupling at this scale, or equivalently by $\Lambda_{Q C D}$, is one of the above mentioned parameters of the theory and has to be taken from experiment.

The RD equations play an important role in our understanding of Quantum Chromodynamics and the strong interactions. The beta function and the quarks mass anomalous dimension are among the most prominent objects for QCD RD equations. The calculation of the one-loop $\beta$-function in QCD has lead to the discovery of asymptotic freedom in this model and to the establishment of QCD as the theory of strong interactions [t Hooft, 1972, Gross, Wilczek, 1973, Politzer, 1973].

The MS-scheme ["t Hooft, 1973] belongs to the class of massless schemes where the $\beta$-function does not depend on masses of the theory and the first two coefficients of the $\beta$-function are schemeindependent.

The Lagrangian of QCD with massive quarks in the covariant gauge is

$$
\begin{aligned}
& L=-\frac{1}{4} F_{\mu v}^{a} F^{a \mu v}+\bar{q}_{n}\left(i \gamma D-m_{n}\right) q_{n} \\
& -\frac{1}{2 \xi}(\partial A)^{2}+\partial^{\mu} \bar{c}^{a}\left(\partial_{\mu} c^{a}+g f^{a b c} A_{\mu}^{b} c^{c}\right) \\
& F_{\mu \nu}^{a}=\partial_{\mu} A_{v}^{a}-\partial_{v} A_{\mu}^{a}+g f^{a b c} A_{\mu}^{b} A_{v}^{c},\left(D_{\mu}\right)_{k l}=\delta_{k l} \partial_{\mu}-i g t_{k l}^{a} A_{\mu}^{a},
\end{aligned}
$$

$A_{\mu}^{a}, a=1, \ldots, N_{c}^{2}-1$ are gluon; $q_{n}, n=1, \ldots, n_{f}$ are quark; $c^{a}$ are ghost fields; $\xi$ is gauge parameter; $t^{a}$ are generators of fundamental representation and $f^{a b c}$ are structure constants of the Lie 
algebra $\left[t^{a}, t^{b}\right]=i f^{a b c} t^{c}$, we consider an arbitrary compact semi-simple Lie group G. For QCD, $G=S U\left(N_{c}\right), N_{c}=3$.

The RD equation for the coupling constant is

$$
\begin{aligned}
& \dot{a}=\beta(a)=\beta_{2} a^{2}+\beta_{3} a^{3}+\beta_{4} a^{4}+\beta_{5} a^{5}+O\left(a^{6}\right), \\
& a=\frac{\alpha_{s}}{4 \pi}=\left(\frac{g}{4 \pi}\right)^{2}, \int_{a_{0}}^{a} \frac{d a}{\beta(a)}=t-t_{0}=\ln \frac{\mu^{2}}{\mu_{0}^{2}},
\end{aligned}
$$

$\mu$ is the 't Hooft unit of mass, the renormalization point in the MS-scheme.

To calculate the $\beta$-function we need to calculate the renormalization constant $Z$ of the coupling constant, $a_{b}=Z a$, where $a_{b}$ is the bare (unrenormalized) charge. The expression of the $\beta$-function can be obtained in the following way

$$
\begin{aligned}
& 0=d\left(a_{b} \mu^{2 \varepsilon}\right) / d t=\mu^{2 \varepsilon}\left(\varepsilon Z a+\frac{\partial(Z a)}{\partial a} \frac{d a}{d t}\right) \\
& \Rightarrow \frac{d a}{d t}=\beta(a, \varepsilon)=\frac{-\varepsilon Z a}{\frac{\partial(Z a)}{\partial a}}=-\varepsilon a+\beta(a), \beta(a)=a \frac{d}{d a}\left(a Z_{1}\right)
\end{aligned}
$$

where

$$
\beta(a, \varepsilon)=\frac{D-4}{2} a+\beta(a)
$$

is $D$-dimensional $\beta$-function and $Z_{1}$ is the residue of the first pole in $\varepsilon$ expansion

$$
Z(a, \varepsilon)=1+Z_{1} \varepsilon^{-1}+\ldots+Z_{n} \varepsilon^{-n}+\ldots
$$

Since $Z$ does not depend explicitly on $\mu$, the $\beta$-function is the same in all MS-like schemes, i.e. within the class of renormalization schemes which differ by the shift of the parameter $\mu$. Note that, presentation of $Z$ in the form of expansion (5.5) is formal. If we take $\varepsilon=1 / p$ we can give the expansion p-adic sense. So, we will have renormalization factors $Z$ as analitic functions of p-adic argument.

For quark anomalous dimension, $\mathrm{RD}$ equation is

$$
\begin{aligned}
& \dot{b}=\gamma(a)=\gamma_{1} a+\gamma_{2} a^{2}+\gamma_{3} a^{3}+\gamma_{4} a^{4}+O\left(a^{5}\right), \\
& b(t)=b_{0}+\int_{t_{0}}^{t} d t \gamma(a(t))=b_{0}+\int_{a_{0}}^{a} d a \gamma(a) / \beta(a) .
\end{aligned}
$$

To calculate the quark mass anomalous dimension $\gamma(g)$ we need to calculate the renormalization constant $Z_{m}$ of the quark mass $m_{b}=Z_{m} m, m_{b}$ is the bare (unrenormalized) quark mass. Than we find the function $\gamma(g)$ in the following way

$$
\begin{aligned}
& 0=\dot{m}_{b}=\dot{Z}_{m} m+Z_{m} \dot{m}=Z_{m} m\left(\left(\ln Z_{m}\right)^{\cdot}+(\ln m)^{\circ}\right) \\
& \Rightarrow \gamma(a)=-\frac{d \ln Z_{m}}{d t}=\dot{b}=-\frac{d \ln Z_{m}}{d a} \frac{d a}{d t}=-\frac{d \ln Z_{m}}{d a}(-\varepsilon a+\beta(a)) \\
& =a \frac{d Z_{m 1}}{d a}, b=-\ln Z_{m}=\ln \frac{m}{m_{b}},
\end{aligned}
$$

where RD equation in $D$-dimension is

$$
\dot{a}=-\varepsilon a+\beta(a)=\beta_{1} a+\beta_{2} a^{2}+\ldots
$$


and $Z_{m 1}$ is the coefficient of the first pole in the $\varepsilon$-expantion of the $Z_{m}$ in $M S$-scheme

$$
Z_{m}(\varepsilon, g)=1+Z_{m 1}(g) \varepsilon^{-1}+Z_{m 2}(g) \varepsilon^{-2}+\ldots
$$

Since $Z_{m}$ does not depend explicitly on $\mu$ and $m$, the $\gamma_{m}$-function is the same in all MS-like schemes.

\subsection{Reparametrization and General Method of Solution of the RD Equation}

RD equation,

$$
\dot{a}=\beta_{1} a+\beta_{2} a^{2}+\ldots
$$

can be reparametrized,

$$
\begin{aligned}
& a(t)=f(A(t))=A+f_{2} A^{2}+\ldots+f_{n} A^{n}+\ldots=\sum_{n \geq 1} f_{n} A^{n}, \\
& \dot{A}=b_{1} A+b_{2} A^{2}+\ldots=\sum_{n \geq 1} b_{n} A^{n}, \\
& \dot{a}=\dot{A} f^{\prime}(A)=\left(b_{1} A+b_{2} A^{2}+\ldots\right)\left(1+2 f_{2} A+\ldots+n f_{n} A^{n-1}+\ldots\right) \\
& =\beta_{1}\left(A+f_{2} A^{2}+\ldots+f_{n} A^{n}+\ldots\right)+\beta_{2}\left(A^{2}+2 f_{2} A^{3}+\ldots\right)+\ldots \\
& +\beta_{n}\left(A^{n}+n f_{2} A^{n+1}+\ldots\right)+\ldots \\
& =\beta_{1} A+\left(\beta_{2}+\beta_{1} f_{2}\right) A^{2}+\left(\beta_{3}+2 \beta_{2} f_{2}+\beta_{1} f_{3}\right) A^{3}+ \\
& \quad \ldots+\left(\beta_{n}+(n-1) \beta_{n-1} f_{2}+\ldots+\beta_{1} f_{n}\right) A^{n}+\ldots \\
& =\sum_{n, n_{1}, n_{2} \geq 1} A^{n} b_{n_{1}} n_{2} f_{n_{2}} \delta_{n, n_{1}+n_{2}-1} \\
& \quad{ } \quad{ }_{n} \quad A^{n} \beta_{m} f_{1}^{m_{1}} \ldots f_{k}^{m_{k}} f\left(n, m, m_{1}, \ldots, m_{k}\right), \\
& \quad \sum_{n, m \geq 1 ; m_{1}, \ldots, m_{k} \geq 0} \\
& f\left(n, m, m_{1}, \ldots, m_{k}\right)=\frac{m !}{m_{1} ! \ldots m_{k} !} \delta_{n, m_{1}+2 m_{2}+\ldots+k m_{k}} \boldsymbol{\delta}_{m, m_{1}+m_{2}+\ldots+m_{k},}, \\
& b_{1}=\beta_{1}, b_{2}=\beta_{2}+f_{2} \beta_{1}-2 f_{2} b_{1}=\beta_{2}-f_{2} \beta_{1}, \\
& b_{3}=\beta_{3}+2 f_{2} \beta_{2}+f_{3} \beta_{1}-2 f_{2} b_{2}-3 f_{3} b_{1}=\beta_{3}+2\left(f_{2}^{2}-f_{3}\right) \beta_{1}, \\
& b_{4}=\beta_{4}+3 f_{2} \beta_{3}+f_{2}^{2} \beta_{2}+2 f_{3} \beta_{2}-3 f_{4} b_{1}-3 f_{3} b_{2}-2 f_{2} b_{3}, \ldots \\
& b_{n}=\beta_{n}+\ldots+\beta_{1} f_{n}-2 f_{2} b_{n-1}-\ldots-n f_{n} b_{1}, \ldots
\end{aligned}
$$

so, by reparametrization, beyond the critical dimension $\left(\beta_{1} \neq 0\right)$ we can change any coefficient but $\beta_{1}$.

We can fix any higher coefficient with zero value, if we take

$$
f_{2}=\frac{\beta_{2}}{\beta_{1}}, f_{3}=\frac{\beta_{3}}{2 \beta_{1}}+f_{2}^{2}, \ldots, f_{n}=\frac{\beta_{n}+\ldots}{(n-1) \beta_{1}}, \ldots
$$

In the critical dimension of space-time, $\beta_{1}=0$, and we can change by reparametrization any coefficient but $\beta_{2}$ and $\beta_{3}$.

From the relations ([.]3)), in the critical dimenshion $\left(\beta_{1}=0\right)$, we find that, we can define the minimal form of the RD equation

$$
\dot{A}=\beta_{2} A^{2}+\beta_{3} A^{3}
$$


We can solve (5.15) as implicit function,

$$
u^{\beta_{3} / \beta_{2}} e^{-u}=c e^{\beta_{2} t}, u=\frac{1}{A}+\frac{\beta_{3}}{\beta_{2}}
$$

then, as in the noncritical case, explicit solution will be given by reparametrization representation (5.) [Makhaldiani, 2013].

If we know somehow the coefficients $\beta_{n}$, e.g. for first several exact and for others asymptotic values (see e.g. [Kazakov, Shirkov, 1980]) than we can construct reparametrization function (5.CI) and find the dynamics of the running coupling constant. This is similar to the action-angular canonical transformation of the analytic mechanics (see e.g. [Faddeev, Takhtajan, 1990]).

Statement: The reparametrization series for $a$ is p-adically convergent, when $\beta_{n}$ and $A$ are rational numbers.

Let us take the the anomalous dimension of some quantity

$$
\gamma(a)=\gamma_{1} a+\gamma_{2} a^{2}+\gamma_{3} a^{3}+\ldots
$$

and make reparametrization

$$
\begin{gathered}
a=f(A)=A+f_{2} A^{2}+f_{3} A^{3}+\ldots \\
\gamma(a)=\gamma_{1}\left(A+f_{2} A^{2}+f_{3} A^{3}+\ldots\right)+\gamma_{2}\left(A^{2}+2 f_{2} A^{3}+\ldots\right)+\gamma_{3}\left(A^{3}+\ldots\right)+\ldots \\
=\Gamma_{1} A+\Gamma_{2} A^{2}+\Gamma_{3} A^{3}+\ldots \\
\Gamma_{1}=\gamma_{1}, \Gamma_{2}=\gamma_{2}+\gamma_{1} f_{2}, \Gamma_{3}=\gamma_{3}+2 \gamma_{2} f_{2}+\gamma_{1} f_{3}, \ldots
\end{gathered}
$$

When $\gamma_{1} \neq 0$, we can take $\Gamma_{n}=0, n \geq 2$, if we define $f_{n}$ as

$$
f_{2}=-\frac{\gamma_{2}}{\gamma_{1}}, f_{3}=-\frac{\gamma_{3}+2 \gamma_{2} f_{2}}{\gamma_{1}}=-\frac{\gamma_{3}-2 \gamma_{2}^{2} / \gamma_{1}}{\gamma_{1}}, \ldots
$$

So, we get the exact value for the anomalous dimension

$$
\gamma(A)=\gamma_{1} A=\gamma_{1} f^{-1}(a)=\gamma_{1}\left(a+\gamma_{2} / \gamma_{1} a^{2}+\gamma_{3} / \gamma_{1} a^{3}+\ldots:\right)
$$

\subsection{Parton Model, Valence Quarks and $\alpha_{s}=2$}

The parton distribution functions (PDFs) of the proton are of fundamental importance for modern particle physics. They describe our current knowledge about the internal structure and symmetries of this basic building block of matter and represent an important baseline for nuclear structure and deconfinement studies, they also enter in the theoretical description of all hadron collider experiments, precision determinations of Standard Model parameters and new physics searches, in particular those at the energy frontier of the Large Hadron Collider (LHC), as only partonic, but not hadronic cross sections are calculable in perturbative QCD.

While it has been well established in the perturbative regime at high energies, QCD still lacks a comprehensive solution at low and intermediate energies, even 40 years after its invention. In order to deal with the wealth of non-perturbative phenomena, various approaches are followed with limited validity and applicability. This is especially also true for lattice QCD, various functional 
methods, or chiral perturbation theory, to name only a few. In neither one of these approaches the full dynamical content of QCD can yet be included. Basically, the difficulties are associated with a relativistically covariant treatment of confinement and the spontaneous breaking of chiral symmetry, the latter being a well-established property of QCD at low and intermediate energies. As a result, most hadron reactions, like resonance excitations, strong and electroweak decays etc., are nowadays only amenable to models of QCD. Most famous is the constituent-quark model (CQM, 1964), which essentially relies on a limited number of effective degrees of freedom with the aim of encoding the essential features of low- and intermediate-energy QCD.

The CQM has a long history, and it has made important contributions to the understanding of many hadron properties, think only of the fact that the systematization of hadrons in the standard particle-data base follows the valence-quark picture. Namely the $Q$ dependence of the nucleon form factor corresponds to three-constituent picture of the nucleon and is well described by the simple equation [Brodsky, Farrar,1973], [Matveev, Muradyan,Tavkhelidze, 1973]

$$
F\left(Q^{2}\right) \sim\left(Q^{2}\right)^{-2}
$$

It was noted [Doloshin, Ter-Martyrosian, 1984] that parton densities given by the following solution

$$
\begin{aligned}
& M_{2}\left(Q^{2}\right)=\frac{3}{25}+\frac{2}{3} \omega^{32 / 81}+\frac{16}{75} \omega^{50 / 81} \\
& \bar{M}_{2}\left(Q^{2}\right)=M_{2}^{s}\left(Q^{2}\right)=\frac{3}{25}-\frac{1}{3} \omega^{32 / 81}+\frac{16}{75} \omega^{50 / 81} \\
& M_{2}^{G}\left(Q^{2}\right)=\frac{16}{25}\left(1-\omega^{50 / 81}\right), \\
& \omega=\frac{\alpha_{s}\left(Q^{2}\right)}{\alpha_{s}\left(m^{2}\right)}, Q^{2} \in(5,20) G e V^{2}, b=9, \alpha_{s}\left(Q^{2}\right) \simeq 0.2
\end{aligned}
$$

of the Altarelli-Parisi equation

$$
\begin{aligned}
& \dot{M}=A M, M^{T}=\left(M_{2}, \bar{M}_{2}, M_{2}^{s}, M_{2}^{G}\right), \\
& M_{2}=\int_{0}^{1} d x x(u(x)+d(x)), \bar{M}_{2}=\int_{0}^{1} d x x(\bar{u}(x)+\bar{d}(x)), \\
& M_{2}^{s}=\int_{0}^{1} d x x(s(x)+\bar{s}(x)), M_{2}^{G}=\int_{0}^{1} d x x G(x), \dot{M}=Q^{2} \frac{d M}{d Q^{2}} \\
& A=-a\left(Q^{2}\right)\left(\begin{array}{cccc}
32 / 9 & 0 & 0 & -2 / 3 \\
0 & 32 / 9 & 0 & -2 / 3 \\
0 & 0 & 32 / 9 & -2 / 3 \\
-32 / 9 & -32 / 9 & -32 / 9 & 2
\end{array}\right), a=\left(\frac{g}{4 \pi}\right)^{2}
\end{aligned}
$$

with the following "valence quark" initial condition at a scale $m$

$$
M_{2}\left(m^{2}\right)=1, \bar{M}_{2}=M_{2}^{s}=M_{2}^{G}\left(m^{2}\right)=0, \alpha_{s}\left(m^{2}\right)=2
$$

gives the experimental values

$$
M_{2}=0.44, \bar{M}_{2}=M_{2}^{s}=0.04, M_{2}^{G}=0.48
$$


Indeed. The APE has the following form

$$
\begin{aligned}
& \dot{x}_{1}=a\left(k x_{1}-b x_{4}\right), \\
& \dot{x}_{2}=a\left(k x_{2}-b x_{4}\right), \\
& \dot{x}_{3}=a\left(k x_{3}-b x_{4}\right), \\
& \dot{x}_{4}=-a k\left(x_{1}+x_{2}+x_{3}\right)+a c x_{4}, \\
& k=32 / 9, b=2 / 3, c=2
\end{aligned}
$$

One of the integral of motion of this system is

$$
H=x_{1}+x_{2}+x_{3}+x_{4}=1
$$

Indeed,

$$
\dot{H}=a(c-3 b)=0 .
$$

The physical meaning of the integral is the statement that the momentum of the nucleon is equal to the sum of the constituent quark and gluon momenta. Now, the equation for $x_{4}=x$, using the integral o motion, we reduce to

$$
\begin{aligned}
& \dot{x}=a((k+c) x-k) \Rightarrow \int \frac{d x}{(k+c) x-k}=a d t=-\frac{d a}{\beta_{1} a} \\
& \Rightarrow x_{4}\left(Q^{2}\right)=\frac{k}{k+c}+\left(x_{40}-\frac{k}{k+c}\right) \omega^{(k+c) / \beta_{1}}, t=\ln \frac{Q^{2}}{M^{2}}, \omega=\frac{\alpha_{s}\left(Q^{2}\right)}{\alpha_{s}\left(M^{2}\right)} \\
& x_{1}+x_{2}+x_{3}=3 \bar{x}=\frac{c}{k+c}+\left(3 \bar{x}_{0}-\frac{c}{a+c}\right) \omega^{(k+c) / \beta_{1}}, 3 \bar{x}_{0}+x_{40}=1
\end{aligned}
$$

Then,

$$
\begin{aligned}
& x_{1}-x_{2}=\left(x_{10}-x_{20}\right) \omega^{k / \beta_{1}} \rightarrow 0, x_{1}-x_{3}=\left(x_{10}-x_{30}\right) \omega^{k / \beta_{1}} \rightarrow 0, \\
& x_{1}, x_{2}, x_{3} \rightarrow \bar{x}=\frac{c}{3(k+c)}=\frac{3}{25}, x_{4} \rightarrow \frac{k}{k+c}=\frac{16}{25}, Q^{2} \gg M^{2}
\end{aligned}
$$

The solution of the system is

$$
\begin{aligned}
& x_{n}=\bar{x}+c_{n} \omega^{k / \beta_{1}}+d_{n} \omega^{(k+c) / \beta_{1}}, n=1,2,3, \\
& x_{4}\left(Q^{2}\right)=\frac{k}{k+c}+\left(x_{40}-\frac{k}{k+c}\right) \omega^{(k+c) / \beta_{1}}, \\
& d_{1}=d_{2}=d_{3}=d, c_{1}+c_{2}+c_{3}=0
\end{aligned}
$$

For the VQM,

$$
\begin{aligned}
& x_{10}=1 \Rightarrow \bar{x}+c_{1}+d=1, \\
& x_{20}=x_{30}=0 \Rightarrow c_{2}=c_{3}=c \Rightarrow x_{2}=x_{3}, c=-c_{1} / 2, d=1-\bar{x}-c_{1}, \bar{x}+c+d=0, \\
& c_{1}=\frac{2}{3}, \bar{x}=\frac{3}{25}, d=1-\frac{3}{25}-\frac{2}{3}=\frac{16}{75}, \frac{k}{\beta_{1}}=\frac{32}{81}, \frac{k+c}{\beta_{1}}=\frac{50}{81}, \beta_{1}=9, x_{40}=0
\end{aligned}
$$

So, for valence quark model (VQCD), $\alpha_{s}\left(m^{2}\right)=2$. We have seen, that for $\pi \rho N$ model $\alpha_{\pi \rho N}=$ 3, and for $\pi N$ model $\alpha_{\pi N}=13$. It is nice that $\alpha_{s}^{2}+\alpha_{\pi \rho N}^{2}=\alpha_{\pi N}$. This relation can be seen, e.g., by considering pion propagator in the low energy $\pi N$ model and in superposition of higher energy VQCD and $\pi \rho N$ models.

Note that to $\alpha_{s}=2$ corresponds

$$
g=\sqrt{4 \pi \alpha_{s}}=5.013=5+
$$




\subsection{Renormdynamic functions (RDF)}

We will call RDF functions $g_{n}=f_{n}(t)$, which are solutions of the RD motion equations

$$
\dot{g}_{n}=\beta_{n}(g), 1 \leq n \leq N .
$$

In the simplest case of one coupling constant, the function $g=f(t)$, is constant $g=g_{c}$ when $\beta\left(g_{c}\right)=0$, or is invertible (monotone). Indeed,

$$
\dot{g}=f^{\prime}(t)=f^{\prime}\left(f^{-1}(g)\right)=\beta(g) .
$$

Each monotone interval ends by UV and IR fixed points and describes corresponding phase of the system.

Note that, the simplest case of the classical dynamics, the Hamiltonian system with one degree of freedom, is already two dimensional, so we have not an analog of one charge renormdynamics. Then the regular Hamiltonian systems of the classical mechanics are defined on the even dimensional phase space, so there is not an analog of the three dimensional renormdynamics for the coupling constants of the SM.

The fixed points of renormdynamics belong to the set of zeros of the polynomial system of equations $\beta_{n}(g)=0,1 \leq n \leq N$, in the perturbative renormdynamics. Describing qualitative and numerical properties of the set, finding the number and multiplicity, evaluation and finding the numerical values of the zeros is the task of contemporary algebraic and computational geometry [Cox, Little, O'Shea 1998].

\subsection{Nonperturbative renormdynamic functions}

Based on real experiments and computer simulations, quantum gauge theory in four dimensions is believed to have a mass gap. This is one of the most fundamental facts that makes the Universe the way it is.

In the lattice (gauge) theory approach to the renormdynamics (see, e.g. [Makhaldiani, 1986]), coupling constant dynamics were calculated for $S U$ (3) Yang-Mills model [Bogolubsky et al, 2009]. The result is in agreement with perturbative calculations at small scales; at an intermediate scale the coupling constant reaches its $\operatorname{maximum}\left(\alpha_{s} \simeq 2\right)$; than decrease. So, at the maximum, we may have nontrivial zero of the $\beta$-function, which corresponds to the conformal invariance of the gluodynamics at this point. Beyond this point we have another phase, strong coupling phase with decreasing coupling constant similar (identical?!) to the abelian (monopole?) theory.

Note that, in the case of the two coupling constants,

$$
\begin{aligned}
& \dot{g}_{1}=\beta_{1}\left(g_{1}, g_{2}\right), \\
& \dot{g}_{2}=\beta_{2}\left(g_{1}, g_{2}\right),
\end{aligned}
$$

we can reformulate RD as

$$
\begin{aligned}
& g_{1} \equiv g ; g_{2}=f_{2}(t) \equiv \tau, \\
& \frac{d g_{1}}{d g_{2}}=\frac{d g}{d \tau} \equiv \dot{g}=\beta(g, \tau)=\frac{\beta_{1}(g, \tau)}{\beta_{2}(g, \tau)}
\end{aligned}
$$


and RDF must fulfil corresponding restrictions. E.g. if

$$
g_{1}=f_{1}(t)=g=f(\tau)=f\left(f_{2}(t)\right), g_{2}=f_{2}(t)=\tau
$$

So, if we approximate the form of the curve near maximum as

$$
a(t)=a_{c}-b\left|t-t_{c}\right|^{n}
$$

for the $\beta$-function we obtain

$$
\dot{a}=\beta(a, t)=\operatorname{sign}\left(t_{c}-t\right) \operatorname{bn}\left(\frac{a_{c}-a}{b}\right)^{\frac{n-1}{n}} .
$$

Of course this is not usual $\beta$-function, function of $a$ only. It depends also on $t$. For $t>t_{c}$ we have perturbative phase. For $n>1, \beta\left(a_{c}, t\right)=0$. Explicit dependence on time variable in one coupling case indicates on implicit two coupling case.

\subsection{Nonperturbative Renormdynamics, AdS/CFT Duality}

The AdS/CFT duality provides a gravity description in a $(d+1)$-dimensional AdS spacetime in terms of a flat d-dimensional conformally-invariant quantum field theory defined at the AdS asymptotic boundary [Maldacena, 1999], [Gubser,Klebanov,Polyakov, 1998], [Witten, 1998]. Thus, in principle, one can compute physical observables in a strongly coupled gauge theory in terms of a classical gravity theory. The $\beta$-function for the nonperturbative effective coupling obtained from the LF holographic mapping in a positive dilaton modified AdS background is [Brodsky, de Tèramond, Deur, 2010]

$$
\begin{aligned}
& \beta\left(\alpha_{A d S}\right)=\frac{d \alpha_{A d S}}{\ln Q^{2}}=-\frac{Q^{2}}{4 k^{2}} \alpha_{A d S}\left(Q^{2}\right) \\
& =\alpha_{A d S}\left(Q^{2}\right) \ln \frac{\alpha_{A d S}\left(Q^{2}\right)}{\alpha(0)} \leq 0
\end{aligned}
$$

where the physical QCD running coupling in its nonperturbative domain is

$$
\alpha_{A d S}\left(Q^{2}\right)=\alpha(0) e^{-Q^{2} / 4 k^{2}}
$$

So, this renormdynamics of QCD interpolates between IR fixed point $\alpha(0)$, which we take as $\alpha(0)=2$, and UV fixed point $\alpha(\infty)=0$.

For the QCD running coupling [Diakonov, 2003]

$$
\alpha\left(q^{2}\right)=\frac{4 \pi}{9 \ln \left(\frac{q^{2}+m_{g}^{2}}{\Lambda^{2}}\right)}
$$

where $m_{g}=0.88 \mathrm{GeV}, \Lambda=0.28 \mathrm{GeV}$, the $\beta$-function of renormdynamics is

$$
\begin{aligned}
& \beta\left(q^{2}\right)=-\frac{\alpha^{2}}{k}\left(1-c \exp \left(-\frac{k}{\alpha}\right)\right)=-\frac{\alpha^{2}}{k}+\frac{c \alpha^{2}}{k} \exp \left(-\frac{k}{\alpha}\right), \\
& k=\frac{4 \pi}{9}=1.40, c=\frac{m_{g}^{2}}{\Lambda^{2}}=(3.143)^{2}=9.88
\end{aligned}
$$


for nontrivial (IR) fixed point we have

$$
\alpha_{I R}=\frac{k}{\ln c}=0.61
$$

For $\alpha(0)=2$, we predict the gluon mass as

$$
m_{g}=\Lambda e^{\frac{k}{2 \alpha(0)}}=1.42 \Lambda=m_{N} / 3, \Lambda=220 \mathrm{MeV} .
$$

The ghost-gluon interaction in Landau gauge has been determined either from DSEs

[Zwanziger, 2002], [Lerche,von Smekal, 2002], or the Exact Renormalization Group Equations (ERGEs) [Pawlowski et al, 2004], [Fischer,Gies, 2004] and yield an IR fixed point

$$
\begin{aligned}
& \alpha(0)=\frac{2 \pi}{3 N_{c}} \frac{\Gamma(3-2 k) \Gamma(3+k) \Gamma(1+k)}{\Gamma(2-k)^{2} \Gamma(2 k)}=\frac{8.9115}{N_{c}}=2.970, \\
& N_{c}=3, k=(93-\sqrt{1201}) / 98=0.5954
\end{aligned}
$$

Note that, from this formula for $k=0.6036$ we have $\alpha(0)=3$ and for $k=0.36$ we have $\alpha(0)=2$.

\subsection{Scale and Conformal Symmetries and Zeros of the Renormdynamic $\beta$-functions}

In a renormalizable or superrenormalizable field theory one can use different mass-independent renormalization schemes. Each scheme will give a different definition of the renormalized coupling constant $g$ and the corresponding Gell- Mann-Low (GML) $\beta$ - functions that appears in the renormalization-group equations.

The main motion equation of the renormdynamics

$$
\dot{a}=\beta_{a}
$$

has fixed points $a_{c}$ in the zeros of the $\beta_{a}=\beta\left(a_{c}\right)=0$. At these points corresponding field theory is scale and conformal symmetric. By reparametrization $a=f(A)$,

$$
f^{\prime}(A) \dot{A}=\beta_{a}(f(A)) \Rightarrow \dot{A}=\beta_{A}(A)=\beta_{a}(f(A)) / f^{\prime}(A),
$$

we can change the form of the motion equation and particulary we can take the minimal form of the $\beta$ - functions depending only on the reparametrization invariant coefficients, e.g. for QCD in critical $d=4$ dimensions

$$
\dot{a}=\beta_{2} a^{2}+\beta_{3} a^{2},
$$

This case, we have the trivial zero $a_{c}=0$, corresponding to the scale and conformal symmetry of QCD at small scales (Higher energies). There are an opinion that at low energy we have another, the nontrivial fixed point. Personally my believe is that the fixed point is $\alpha_{s}(M)=2$ at the valence quark scale $M \sim 300 \mathrm{MeV}$. But it is obvious that the minimal form of the QCD renormdynamics (5.5]) has not the finite nontrivial fixed point! How I can talk about the fixed point?

Thing is that, the original (complete, physical, if you like) $\beta$ - function and the minimal one are connected as

$$
\beta_{a}=f^{\prime}(A) \beta_{A}
$$


so, when the minimal $\beta$ - function has not the nontrivial fixed point-zero, that fixed point is given by critical point of the reparametrization function, $f(A), f^{\prime}\left(A_{c}\right)=0$. Then, when the minimal $\beta$ function has not the nontrivial zero, but we know somehow the fixed point, we can consider by corresponding reparametrization a next to the minimal forms of the $\beta$-function which will have the nontrivial fixed point.

If we do not know the value of the nontrivial fixed point, we can find its approximation value from the zeros of the reparametrization function $f(A)$, which reduce known approximation value of the $\beta$ - function to the minimal one.

For monotonic function $a=f(A), f^{\prime}(A) \neq 0$ and we can define another time-parameter

$$
d \tau=d t /\left|f^{\prime}(A(t))\right|
$$

The renormdynamic properties of Quantum Chromodynamics were the reason of acceptance of this theory as the theory of strong interactions. The central role played by the QCD $\beta$-function, calculated at the one- ["t Hooft, 1972], [Gross, Wilczek, 1973], [Politzer, 1973], two-[Caswell, 1974], [एones, 1974], [Egorian,Tarasov, 1979], three-[Tarasov,Vladimirov,Zharkov,1980], [Larin,Vermaseren,1993] and finally at the four-loop- van Ritbergen,Vermaseren,Larin, 1997] level, cannot be overestimated in this respect.

The minimal form of the QCD renormdynamics $(\mathrm{RD})$ is

$$
\begin{aligned}
& \dot{x}=-b_{2} x^{2}-b_{3} x^{3}, \\
& b_{2}=11-\frac{2}{3} n, b_{3}=2\left(51-\frac{19}{3} n\right), x=\frac{\alpha_{s}}{4 \pi}=\left(\frac{g}{4 \pi}\right)^{2},
\end{aligned}
$$

where $n$ is the number of the light quarks, e.g. $n=3$ for energy scales less then the mass of the $c$-quark, $m_{c} \simeq 1 \mathrm{GeV}$ but higher than the mass of $s-$ quark, $m_{s} \simeq 100 \mathrm{MeV}$.

\subsection{Two TeV Scale Unification of the Standard Model Coupling Constants}

According to the LEP and Tevatron data, the standard model coupling constants at the Z-boson mass scale take the values (see, e.g. [Kazakov, 2004])

$$
\begin{aligned}
& \alpha_{1}\left(m_{Z}\right)=0.017, \alpha_{1}\left(m_{Z}\right)^{-1}=58.8 \\
& \alpha_{2}\left(m_{Z}\right)=0.034, \alpha_{2}\left(m_{Z}\right)^{-1}=29.4 \\
& \alpha_{3}\left(m_{Z}\right)=0.118, \alpha_{3}\left(m_{Z}\right)^{-1}=8.47 \\
& m_{Z}=91.1875 \mathrm{GeV}
\end{aligned}
$$

Note that $\alpha_{2}\left(m_{Z}\right)=2 \alpha_{1}\left(m_{Z}\right)$.

Our aim is to consider RD equation in critical dimension for weak interaction part of the $\mathrm{SM}\left(\varepsilon_{2}=0\right)$; RD equations for the electromagnetic and strong interaction parts beyond critical dimension $\left(\varepsilon_{1}, \varepsilon_{3} \neq 0\right)$; reach unification (equality) of the three couplings at the $\mathrm{TeV}$ scale in the point $\alpha_{u}^{-1}=31.0$

The solution of the one loop RD equation beyond critical dimension

$$
\begin{aligned}
& \dot{a}=-\varepsilon a+k a^{2}, \\
& a=\frac{\alpha}{4 \pi}=\left(\frac{g}{4 \pi}\right)^{2}, t=\ln \frac{Q^{2}}{m_{Z}^{2}},
\end{aligned}
$$


is

$$
\begin{aligned}
& a_{n}(t)^{-1}=\frac{k_{n}}{\varepsilon}+c_{n} e^{\varepsilon_{n} t}, n=1,3 \\
& c_{n}=a_{n}\left(m_{Z}\right)^{-1}-\frac{k_{n}}{\varepsilon_{n}}, \\
& k_{n}=\left(\frac{41}{10},-7\right) .
\end{aligned}
$$

The solution of the RD equation in critical dimension

$$
\dot{a}_{2}=k_{2} a_{2}^{2}, k_{2}=-\frac{19}{6}
$$

is

$$
a_{2}^{-1}(t)=a_{2}^{-1}\left(m_{Z}\right)+k_{2} t
$$

From the last expression, having unification value, $\alpha_{2}^{-1}\left(t_{u}\right)=\alpha_{u}^{-1}=31.0$ we define the unification scale

$$
\begin{aligned}
& t_{u}=\left(a_{2}^{-1}\left(t_{u}\right)-a_{2}^{-1}\left(m_{Z}\right)\right) / k_{2} \\
& =4 \pi \times 1.6 \times \frac{6}{19}=6.35, \\
& Q_{u}=23.9 m_{Z}=2182 \mathrm{GeV}, \\
& m_{Z}=91.2 \mathrm{GeV}
\end{aligned}
$$

Solution of the RD equation beyond the critical dimension for electrodynamic constant,

$$
\dot{a}=-\varepsilon a+b a^{2}, b=\frac{41}{10},
$$

is

$$
a^{-1}(t)=\frac{b}{\varepsilon}+\left(a^{-1}\left(m_{Z}\right)-\frac{b}{\varepsilon}\right) e^{\varepsilon t}
$$

The condition of the unification

$$
\left(b \varepsilon^{-1}-a^{-1}\left(t_{u}\right)\right)=\left(b \varepsilon^{-1}-a^{-1}\left(m_{Z}\right)\right) e^{\varepsilon t_{u}}
$$

defines the value $\varepsilon_{1}=-0.093$ Unification takes place in dimension $d=4-2 \varepsilon_{1}=4.186$

For the strong coupling constant beyond the critical dimension,

$$
\dot{a}=-\varepsilon a-b a^{2}, b=7,
$$

the solution is

$$
a^{-1}(t)=-\frac{b}{\varepsilon}+\left(\frac{b}{\varepsilon}+a^{-1}\left(m_{Z}\right)\right) e^{t \varepsilon}
$$

the unification condition

$$
\left(b \varepsilon^{-1}+a^{-1}\left(t_{u}\right)\right)=\left(b \varepsilon^{-1}+a^{-1}\left(m_{Z}\right)\right) e^{\varepsilon t_{u}}
$$


defines $\varepsilon=0.168$ Unification takes place in the dimension $d=4-2 \varepsilon=3.66$

Let us consider unification at the point $\alpha^{-1}\left(t_{u}\right)=29.0$, the low energy unification,

$$
\begin{aligned}
& t_{u l}=\left(\alpha_{2}^{-1}\left(t_{u l}\right)-a_{2}^{-1}\left(m_{Z}\right)\right) / k_{2} \\
& =-4 \pi \times 0.4 \times \frac{6}{19}=-1.59 \\
& Q_{u l}=0.45 m_{Z}=41.2 \mathrm{GeV}
\end{aligned}
$$

This maybe good indication on the composite nature of the weak interaction bosons with corresponding constituent valence mass $m \sim 40 \mathrm{GeV}$. Note that, $m_{W}, m_{Z} \sim 2 m, m_{H}=125 \mathrm{GeV} \sim 3 m$.

For electrodynamic case unification condition

$$
\frac{41}{10}-4 \pi 29 \varepsilon=\left(\frac{41}{10}-4 \pi 58.8 \varepsilon\right) e^{-1.59 \varepsilon},
$$

gives the values $\varepsilon_{1}=0.453, d_{e l}=3.09=2.09+1$ dimensional space-time.

For strong coupling constant unification condition

$$
7+4 \pi \varepsilon \times 29=(7+4 \pi \varepsilon \times 8.47) e^{-1.59 \varepsilon}
$$

gives $\varepsilon_{3}=-0.8121, d_{s l}=5.624$

5.8 At what scale $\alpha^{-1}=137$ ?

The low energy value of the QED $\alpha^{-1}=137.036$

Let us find the scale at which $\alpha^{-1}=137$ if

$$
\begin{aligned}
& \alpha^{-1}\left(m_{Z}\right)=\frac{5}{3 \cos ^{2} \theta_{W}} \alpha_{1}^{-1}\left(m_{Z}\right)=128.978 \pm 0.027 \simeq 129 \\
& \sin ^{2} \theta_{W}=0.23146 \pm 0.00017 \simeq 0.2315 \\
& \alpha_{1}^{-1}\left(m_{Z}\right)=58.8
\end{aligned}
$$

${ }^{1}$ Now take one loop RD evolution to the 137 ,

$$
\begin{aligned}
& t_{l}=\left(a_{1}^{-1}\left(t_{l}\right)-a_{1}^{-1}\left(m_{Z}\right)\right) / k_{1} \\
& =-4 \pi \times 8 . \times \frac{10}{41}=-24.5, \\
& Q_{l} \simeq 5 \times 10^{-6} m_{Z} \simeq 5 \times 10^{-4} m_{p} \simeq m_{e}
\end{aligned}
$$

There are the following prime numbers less than 137: 131, 127, 113,..., 37, 31, 29, 23, 19, 17, 13, $11,7,5,3,2$. It is interesting to fined corresponding scales - particles.

For the nearest prime number 131, we have

$$
\begin{aligned}
& t_{l}=\left(a_{1}^{-1}\left(t_{l}\right)-a_{1}^{-1}\left(m_{Z}\right)\right) / k_{1} \\
& =-4 \pi \times 2 . \times \frac{10}{41}=-6.13, \\
& Q_{l} \simeq 4.67 \times 10^{-2} m_{Z} \simeq 4.25 \mathrm{GeV}
\end{aligned}
$$

This is $b$ quarks mass $m_{b}=4.2 \mathrm{GeV}$.

\footnotetext{
${ }^{1}$ By the way, $129=3 \times 43,(3+4)^{3}=343$ is the number of my office :)
} 
The bottom quark or $b$ quark, also known as the beauty quark, is a third-generation quark with a charge of $-1 / 3 e$. Although all quarks are described in a similar way by the quantum chromodynamics, the bottom quark's large bare mass (around $4.2 \mathrm{GeV}$, [Beringer et al, 2012] a bit more than four times the mass of a proton), combined with low values of the CKM matrix elements $V_{u b}$ and $V_{c b}$, gives it a distinctive signature that makes it relatively easy to identify experimentally (using a technique called B-tagging). Because three generations of quark are required for $\mathrm{CP}$ violation, mesons containing the bottom quark are the easiest particles to use to investigate the phenomenon; such experiments are being performed at the BaBar, Belle and LHCb experiments. The bottom quark is also notable because it is a product in almost all top quark decays, and is a frequent decay product for the Higgs boson.

The bottom quark was theorized in 1973 by physicists Makoto Kobayashi and Toshihide Maskawa to explain CP violation. The name "bottom" was introduced in 1975 by Haim Harari. The bottom quark was discovered in 1977 by the Fermilab E288 experiment team led by Leon M. Lederman, when collisions produced bottomonium. Kobayashi and Maskawa won the 2008 Nobel Prize in Physics for their explanation of CP-violation.

The bottom quark can decay into either an up or charm quark via the weak interaction. Both these decays are suppressed by the CKM matrix, making lifetimes of most bottom particles $(\sim$ $\left.10^{-12} s\right)$ somewhat higher than those of charmed particles $\left(\sim 10^{-13} s\right)$, but lower than those of strange particles (from $\sim 10^{-10} s$ to $\sim 10^{-8} s$ ).

To the next to the nearest prime 127 , we obtain

$$
\begin{aligned}
& t_{l}=\left(a_{1}^{-1}\left(t_{l}\right)-a_{1}^{-1}\left(m_{Z}\right)\right) / k_{1} \\
& =4 \pi \times 2 . \times \frac{10}{41}=6.13 \\
& Q_{l} \simeq 1161 m_{Z} \simeq 106 \mathrm{TeV}
\end{aligned}
$$

For $128=2^{7}$ we have

$$
\begin{aligned}
& t_{l}=\left(a_{1}^{-1}\left(t_{l}\right)-a_{1}^{-1}\left(m_{Z}\right)\right) / k_{1} \\
& =4 \pi \times \frac{10}{41}=3.06 \\
& Q_{l} \simeq 10^{1.5} m_{Z} \simeq 3101 \mathrm{GeV} \sim 3 \mathrm{TeV}
\end{aligned}
$$

For 130 we have

$$
\begin{aligned}
& t_{l}=\left(a_{1}^{-1}\left(t_{l}\right)-a_{1}^{-1}\left(m_{Z}\right)\right) / k_{1} \\
& =-4 \pi \times \frac{10}{41}=-3.06 \\
& Q_{l} \simeq 10^{-1.5} m_{Z} \simeq 2.67 \mathrm{GeV} \sim 2 m_{c}, m_{c}=1.275 \pm 0.025 \mathrm{GeV}
\end{aligned}
$$

For $136=2^{3} \times 17$,

$$
\begin{aligned}
& t_{l}=\left(a_{1}^{-1}\left(t_{l}\right)-a_{1}^{-1}\left(m_{Z}\right)\right) / k_{1} \\
& =-4 \pi \times 7 \frac{10}{41}=-21.4548, \\
& Q_{l} \simeq 2.2^{-5} m_{Z} \simeq 2 \mathrm{MeV} \sim m_{u}, m_{u}=2.3^{-0.5}+0.7
\end{aligned}
$$


For $135=3^{3} \times 5$,

$$
\begin{aligned}
& t_{l}=\left(a_{1}^{-1}\left(t_{l}\right)-a_{1}^{-1}\left(m_{Z}\right)\right) / k_{1} \\
& =-4 \pi \times 6 \frac{10}{41}=-18.3898 \\
& Q_{l} \simeq 1.02 \times 10^{-4} m_{Z} \simeq 9.24 \mathrm{MeV} \sim 2 m_{d}, m_{d}=4.8_{+0.7}^{-0.3}
\end{aligned}
$$

For $134=2^{3} \times 67$,

$$
\begin{aligned}
& t_{l}=\left(a_{1}^{-1}\left(t_{l}\right)-a_{1}^{-1}\left(m_{Z}\right)\right) / k_{1} \\
& =-4 \pi \times 5 \frac{10}{41}=-15.3248 \\
& Q_{l} \simeq 4.7 \times 10^{-4} m_{Z} \simeq 42.8 \mathrm{MeV} \sim m_{s} / 2, m_{s}=95 \pm 5 \mathrm{MeV}
\end{aligned}
$$

For $133=2^{4} \times 7$

$$
\begin{aligned}
& t_{l}=\left(a_{1}^{-1}\left(t_{l}\right)-a_{1}^{-1}\left(m_{Z}\right)\right) / k_{1} \\
& =-4 \pi \times 4 \frac{10}{41}=-12.2599, \\
& Q_{l} \simeq 2.18 \times 10^{-3} m_{Z} \simeq 198.1 \mathrm{MeV} \sim 2 m_{s}, m_{s}=95 \pm 5 \mathrm{MeV}
\end{aligned}
$$

For $132=2^{2} \times 3 \times 11$,

$$
\begin{aligned}
& t_{l}=\left(a_{1}^{-1}\left(t_{l}\right)-a_{1}^{-1}\left(m_{Z}\right)\right) / k_{1} \\
& =-4 \pi \times 3 \frac{10}{41}=-9.19491 \\
& Q_{l} \simeq \times 10^{-2} m_{Z} \simeq 0.91705 \mathrm{GeV} \sim m_{N}, m_{p}=938.272046(21) \mathrm{MeV}
\end{aligned}
$$

\section{Fractal Geometry and Calculus (H) and Some Applications}

Every (good) school boy/girl knows what is

$$
\frac{d^{n}}{d x^{n}}=\partial^{n}=(\partial)^{n}
$$

but what is its following extension

$$
\frac{d^{\alpha}}{d x^{\alpha}}=\partial^{\alpha}, \alpha \in \Re ?
$$

\subsection{Euler, ... Liouville, ... Holmgren, ...}

Let us consider the integer derivatives of the monomials

$$
\begin{aligned}
\frac{d^{n}}{d x^{n}} x^{m} & =m(m-1) \ldots(m-(n-1)) x^{m-n}, n \leq m, \\
& =\frac{\Gamma(m+1)}{\Gamma(m+1-n)} x^{m-n} .
\end{aligned}
$$

L.Euler (1707 - 1783) invented the following definition of the fractal derivatives,

$$
\frac{d^{\alpha}}{d x^{\alpha}} x^{\beta}=\frac{\Gamma(\beta+1)}{\Gamma(\beta+1-\alpha)} x^{\beta-\alpha}
$$


J.Liouville (1809-1882) takes exponents as a base functions,

$$
\frac{d^{\alpha}}{d x^{\alpha}} e^{a x}=a^{\alpha} e^{a x}
$$

The following Cauchy formula

$$
I_{0, x}^{n} f=\int_{0}^{x} d x_{n} \int_{0}^{x_{n-1}} d x_{n-2} \cdots \int_{0}^{x_{2}} d x_{1} f\left(x_{1}\right)=\frac{1}{\Gamma(n)} \int_{0}^{x} d y(x-y)^{n-1} f(y)
$$

permits analytic extension from integer $n$ to complex $\alpha$,

$$
I_{0, x}^{\alpha} f=\frac{1}{\Gamma(\alpha)} \int_{0}^{x} d y(x-y)^{\alpha-1} f(y)
$$

J.H. Holmgren invented (in 1863) the following integral transformation,

$$
D_{c, x}^{-\alpha} f=\frac{1}{\Gamma(\alpha)} \int_{c}^{x}|x-t|^{\alpha-1} f(t) d t .
$$

It is easy to show that

$$
\begin{aligned}
D_{c, x}^{-\alpha} x^{m} & =\frac{\Gamma(m+1)}{\Gamma(m+1+\alpha)}\left(x^{m+\alpha}-c^{m+\alpha}\right), \\
D_{c, x}^{-\alpha} e^{a x} & =a^{-\alpha}\left(e^{a x}-e^{a c}\right)
\end{aligned}
$$

so, $c=0$, when $m+\alpha \geq 0$, in Holmgren's definition of the fractal calculus, corresponds to the Euler's definition, and $c=-\infty$, when $a>0$, corresponds to the Liouville's definition.

Holmgren's definition of the fractal calculus reduce to the Euler's definition for finite $c$, and to the Liouvill's definition for $c=\infty$,

$$
\begin{aligned}
& D_{c, x}^{-\alpha} f=D_{0, x}^{-\alpha} f-D_{0, c}^{-\alpha} f, \\
& D_{\infty, x}^{-\alpha} f=D_{-\infty, x}^{-\alpha} f-D_{-\infty, \infty}^{-\alpha} f .
\end{aligned}
$$

We considered the following modification of the $c=0$ case [Makhaldiani, 2003],

$$
\begin{aligned}
D_{0, x}^{-\alpha} f & =\frac{|x|^{\alpha}}{\Gamma(\alpha)} \int_{0}^{1}|1-t|^{\alpha-1} f(x t) d t,=\frac{|x|^{\alpha}}{\Gamma(\alpha)} B(\alpha, \partial x) f(x) \\
& =|x|^{\alpha} \frac{\Gamma(\partial x)}{\Gamma(\alpha+\partial x)} f(x), f(x t)=t^{x \frac{d}{d x}} f(x) .
\end{aligned}
$$

As an example, consider Euler B-function,

$$
B(\alpha, \beta)=\int_{0}^{1} d x|1-x|^{\alpha-1}|x|^{\beta-1}=\Gamma(\alpha) \Gamma(\beta) D_{01}^{-\alpha} D_{0 x}^{1-\beta} 1=\frac{\Gamma(\alpha) \Gamma(\beta)}{\Gamma(\alpha+\beta)}
$$

We can define also FC as

$$
D^{\alpha} f=\left(D^{-\alpha}\right)^{-1} f=\frac{\Gamma(\partial x+\alpha)}{\Gamma(\partial x)}\left(|x|^{-\alpha} f\right), \partial x=\delta+1, \delta=x \partial
$$


For the Liouville's case,

$$
\begin{gathered}
D_{-\infty, x}^{\alpha} f=\left(D_{-\infty, x}\right)^{\alpha} f=\left(\partial_{x}\right)^{\alpha} f \\
\partial_{x}^{-\alpha} f=\frac{1}{\Gamma(\alpha)} \int_{0}^{\infty} d t t^{\alpha-1} e^{-t \partial_{x}} f(x)=\frac{1}{\Gamma(\alpha)} \int_{0}^{\infty} d t t^{\alpha-1} f(x-t) \\
=\frac{1}{\Gamma(\alpha)} \int_{-\infty}^{x} d t(x-t)^{\alpha-1} f(t)=D_{-\infty, x}^{-\alpha} f .
\end{gathered}
$$

The integrals can be calculated as

$$
D^{-n} f=\left(D^{-1}\right)^{n} f
$$

where

$$
D^{-1} f=x \frac{\Gamma(\partial x)}{\Gamma(1+\partial x)} f=x \frac{1}{\partial x} f=x(\partial x)^{-1} f=(\partial)^{-1} f=\int_{0}^{x} d t f(t) .
$$

Let us consider Weierstrass C.T.W. (1815 - 1897) fractal function

$$
f(t)=\sum_{n \geq 0} a^{n} e^{i\left(b^{n} t+\varphi_{n}\right)}, a<1, a b>1 .
$$

For fractals we have no integer derivatives,

$$
f^{(1)}(t)=i \sum(a b)^{n} e^{i\left(b^{n} t+\varphi_{n}\right)}=\infty
$$

but the fractal derivative,

$$
f^{(\alpha)}(t)=\sum\left(a b^{\alpha}\right)^{n} e^{i\left(b^{n} t+\pi \alpha / 2+\varphi_{n}\right)}
$$

when $a b^{\alpha}=a^{\prime}<1$, is another fractal (6.18).

Question: what if $a b=p$ is prime number? Can we define integer derivatives in this case?

\section{2 p-adic numbers}

In mathematics the $\mathrm{p}$-adic number system for any prime number $p$ extends the ordinary arithmetic of the rational numbers in a way different from the extension of the rational number system to the real and complex number systems. The extension is achieved by an alternative interpretation of the concept of "closeness" or absolute value.

\section{$6.3 \mathrm{p}$ - adic fractal calculus}

Definition of the p-adic norm, ||$_{p}$ for raitional numbers $r \in Q$ is

$$
\begin{gathered}
|r|_{p}=p^{-k}, r \neq 0 ; \\
|0|_{p}=0,
\end{gathered}
$$

where $k=\operatorname{ord}_{p}(r)$ is defined from the following representation of the $\mathrm{r}$

$$
r= \pm p^{k} \frac{m}{n}
$$


integers $\mathrm{m}$ and $\mathrm{n}$ do not contain as factor $\mathrm{p}$.

p-adic analog of the fractal calculus (6.8),

$$
D_{x}^{-\alpha} f=\frac{1}{\Gamma_{p}(\alpha)} \int_{Q_{p}}|x-t|_{p}^{\alpha-1} f(t) d t
$$

where $f(x)$ is a complex function of the $\mathrm{p}$-adic variable $\mathrm{x}$, with $\mathrm{p}$-adic $\Gamma$-function

$$
\Gamma_{p}(\alpha)=\int_{Q_{p}} d t|t|_{p}^{\alpha-1} \chi(t)=\frac{1-p^{\alpha-1}}{1-p^{-\alpha}},
$$

was considered by V.S. Vladimirov [Dladimirov, 1988].

The following modification of p-adic FC is given in [Makhaldiani, 2003]

$$
\begin{aligned}
D_{x}^{-\alpha} f & =\frac{|x|_{p}^{\alpha}}{\Gamma_{p}(\alpha)} \int_{Q_{p}}|1-t|_{p}^{\alpha-1} f(x t) d t \\
& =|x|_{p}^{\alpha} \frac{\Gamma_{p}(\partial|x|)}{\Gamma_{p}(\alpha+\partial|x|)} f(x) .
\end{aligned}
$$

Last expression is applicable for functions of the type $f(x)=f(|x|)$. E.g. the renormalization constants $Z(\lambda)$ of the QFT maybe this type of functions. For a functions of the form

$$
f(x)=\sum a_{n}|x|_{p}^{n}
$$

we have

$$
D_{x}^{-\alpha} f=\sum a_{n} \frac{\Gamma_{p}(n+1)}{\Gamma_{p}(n+1+\alpha)}|x|_{p}^{n+\alpha}
$$

\subsection{Field theory applications of FC}

Let us consider the following action

$$
S=\frac{1}{2} \int_{Q_{v}} d x \Phi(x) D_{x}^{\alpha} \Phi, v=1,2,3,5, \ldots, 29, \ldots, 137, \ldots
$$

$Q_{1}$ is real number field, $Q_{p}, \mathrm{p}$ - prime, are p-adic number fields. In the momentum representation

$$
\begin{aligned}
& S=\frac{1}{2} \int_{Q_{v}} d u \tilde{\Phi}(-u)|u|_{v}^{\alpha} \tilde{\Phi}(u), \Phi(x)=\int_{Q_{v}} d u \chi_{v}(u x) \tilde{\Phi}(u), \\
& D^{-\alpha} \chi_{v}(u x)=|u|_{v}^{-\alpha} \chi_{v}(u x) .
\end{aligned}
$$

The statistical sum of the corresponding quantum theory is

$$
Z_{v}=\int d \Phi e^{-\frac{1}{2} \int \Phi D^{\alpha} \Phi}=\operatorname{det}^{-1 / 2} D^{\alpha}=\left(\prod_{u}|u|_{v}\right)^{-\alpha / 2} .
$$




\subsection{Adeles and Cosmological constant}

The ring of integral adeles $A_{Z}$ is the product of the real numbers $R$ and of all the rings of p-adic integers:

$$
A_{Z}=R \prod_{p=2,3,5, \ldots} Z_{p}=\prod_{p=1,2,3,5, \ldots} Z_{p}, Z_{1} \equiv R
$$

The ring of (rational) adeles $A_{Q}$ is the tensor product

$$
A_{Q}=Q \otimes_{Z} A_{Z}
$$

The ring of (rational) adeles can also be defined as the restricted product

$$
A_{Q}=R \prod_{p}^{\prime} Q_{p}
$$

of all the real numbers and the p-adic completions $Q_{p}$, or in other words as the restricted product of all completions of the rationals. In this case the restricted product means that for an adele $a=\left(a_{1}, a_{2}, a_{3}, a_{5},\right)$ all but a finite number of the $a_{p}$ are p-adic integers.

The group of invertible elements of the adele ring is the idele group. As a locally compact abelian group, the adeles have a nontrivial translation invariant measure. Similarly, the group of ideles has a nontrivial translation invariant measure.

Adels $a \in A$ are constructed by real $a_{1} \in Q_{1}$ and p-adic $a_{p} \in Q_{p}$ numbers (see e.g. [Gelfand et al, 1966])

$$
a=\left(a_{1}, a_{2}, a_{3}, a_{5}, \ldots, a_{p}, \ldots\right),
$$

with restriction that $a_{p} \in Z_{p}=\left\{x \in Q_{p},|x|_{p} \leq 1\right\}$ for all but a finite set $\mathrm{F}$ of primes $\mathrm{p}$.

$A$ is a ring with respect to the componentwise addition and multiplication. A prinsipal adel is a sequence $r=(r, r, \ldots, r, \ldots), r \in Q$-rational number.

Norm on adels is defined as

$$
|a|=\prod_{p \geq 1}\left|a_{p}\right|_{p}
$$

Note that the norm on principal adels is trivial: $|r|=1$, when $r \neq 0$ and $|0|=0$.

Let us consider the following action

$$
S=\frac{1}{2} \int_{Q_{v}} d x \Phi(x) D_{x}^{\alpha} \Phi, v=1,2,3,5, \ldots
$$

In the momentum representation

$$
S=\frac{1}{2} \int_{Q_{v}} d u \tilde{\Phi}(-u)|u|_{v}^{\alpha} \tilde{\Phi}(u),
$$

where

$$
\Phi(x)=\int_{Q_{v}} d u \chi_{v}(u x) \tilde{\Phi}(u)
$$




$$
D^{-\alpha} \chi_{v}(u x)=|u|_{v}^{-\alpha} \chi_{v}(u x)
$$

The statistical sum of the corresponding quantum theory is

$$
Z_{v}=\int d \Phi e^{-\frac{1}{2} \int \Phi D^{\alpha} \Phi}=d e t^{-1 / 2} D^{\alpha}=\left(\prod_{u}|u|_{v}\right)^{-\alpha / 2} .
$$

In the adelic generalization of the model (6.36),

$$
\Phi(x)=\prod_{p \geq 1} \Phi_{p}\left(x_{p}\right), \quad d x=\prod_{p \geq 1} d x_{p}, \quad D_{x}^{\alpha}=\sum_{p \geq 1} D_{x_{p}}^{\alpha},
$$

where by $D_{x_{1}}^{\alpha}$ we denote fractal derivative ([0.9), $x_{1}$ is real and ||$_{1}$ is real norm. If

$$
\int d x_{p}\left|\Phi\left(x_{p}\right)\right|^{2}=1
$$

then

$$
\int d x|\Phi(x)|^{2}=1, \quad S=\sum_{p \geq 1} S_{p}
$$

so

$$
Z=\prod_{p \geq 1} Z_{p}=\prod_{p \geq 1}\left(\prod_{u}|u|_{p}\right)^{-\alpha / 2}=\left(\prod_{u} \prod_{p \geq 1}|u|_{p}\right)^{-\alpha / 2}=1, \quad \lambda \sim \ln Z=0
$$

if $u \in Q$.

\subsection{Fractal qalculus}

The basic object of q-calculus is q-derivative

$$
D_{q} f(x)=\frac{f(x)-f(q x)}{(1-q) x}=\frac{1-q^{x \partial}}{(1-q) x} f(x),
$$

where either $0<q<1$ or $1<q<\infty$. In the limit $q \rightarrow 1, D_{q} \rightarrow \partial_{x}$.

Now we define the fractal q-calculus,

$$
\begin{aligned}
& D_{q}^{\alpha} f(x)=\left(D_{q}\right)^{\alpha} f(x) \\
& =((1-q) x)^{-\alpha}\left(f(x)+\sum_{n \geq 1}(-1)^{n} \frac{\alpha(\alpha-1) \ldots(\alpha-n+1)}{n !} f\left(q^{n} x\right)\right) .
\end{aligned}
$$

For the case $\alpha=-1$, we obtain the integral

$$
D_{q}^{-1} f(x)=(1-q) x\left(1-q^{x \partial}\right)^{-1} f(x)=(1-q) x \sum_{n \geq 0} f\left(q^{n} x\right) .
$$

In the case of $1<q<\infty$, we can give a good analytic sense to these expressions for prime numbers $q=p=2,3,5, \ldots, 29, \ldots, 137, \ldots$ This is an algebra-analytic quantization of the q-calculus and corresponding physical models. Note also, that p-adic calculus is the natural tool for the physical models defined on the fractal( space)s like Bete lattice ( or Brua-Tits trees, in mathematical literature).

Note also a symmetric definition of the calculus

$$
D_{q s} f(x)=\frac{f\left(q^{-1} x\right)-f(q x)}{\left(q^{-1}-q\right) x} f(x) .
$$




\subsection{Fractal finite - difference calculus}

Usual finite difference calculus is based on the following (left) derivative operator

$$
D_{-} f(x)=\frac{f(x)-f(x-h)}{h}=\left(\frac{1-e^{-h \partial}}{h}\right) f(x) .
$$

We define corresponding fractal calculus as

$$
D_{-}^{\alpha} f(x)=\left(D_{-}\right)^{\alpha} f(x) .
$$

In the case of $\alpha=-1$, we have usual finite difference sum as regularization of the Riemann integral

$$
D_{-}^{-1} f(x)=h(f(x)+f(x-h)+f(x-2 h)+\ldots) .
$$

(I believe that) the fractal calculus (and geometry) are the proper language for the quantume (field) theories, and discrete versions of the fractal calculus are proper regularizations of the fractal calculus and field theories.

\subsection{Hypergeometric functions}

A hypergeometric series, in the most general sense, is a power series in which the ratio of successive coefficients indexed by $n$ is a rational function of $n$,

$$
f(x)=\sum_{n \geq 0} a_{n} x^{n}, a_{n+1}=R(n) a_{n}, R(n)=\frac{P(\alpha, n)}{Q(\beta, n)}
$$

so

$$
\begin{aligned}
& P(\alpha, \delta) f(x)=Q(\beta, \delta)(f(x)-f(0)) / x \\
& f(x)-f(0)=x R(\delta) f(x), f(x)=(1-x R(\delta))^{-1} f(0), \delta=x \partial_{x}
\end{aligned}
$$

Hypergeometric functions have many particular special functions as special cases, including many elementary functions, the Bessel functions, the incomplete gamma function, the error function, the elliptic integrals and the classical orthogonal polynomials, because the hypergeometric functions are solutions to the hypergeometric differential equation, which is a fairly general second-order ordinary differential equation.

In a generalization given by Eduard Heine ( 1821 - 1881 ), the ratio of successive terms, instead of being a rational function of $\mathrm{n}$, are considered to be a rational function of $q^{n}$

$$
\begin{aligned}
& f(x)=\sum_{n \geq 0} a_{n} x^{n}, a_{n+1}=R\left(q^{n}\right) a_{n}, R(n)=\frac{P\left(\alpha, q^{n}\right)}{Q\left(\beta, q^{n}\right)}, \\
& P\left(\alpha, q^{\delta}\right) f(x)=Q\left(\beta, q^{\delta}\right)(f(x)-f(0)) / x, \\
& f(x)-f(0)=x R\left(q^{\delta}\right) f(x), f(x)=\left(1-x R\left(q^{\delta}\right)\right)^{-1} f(0), \delta=x \partial_{x}
\end{aligned}
$$

Another generalization, the elliptic hypergeometric series, are those series where the ratio of terms is an elliptic function (a doubly periodic meromorphic function) of $n$.

There are a number of new definitions of hypergeometric series, by Aomoto, Gelfand and others; and applications for example to the combinatorics of arranging a number of hyperplanes in complex $\mathrm{N}$-space. 


\subsection{Hypergeometric field theory (HFT)}

Formal solutions for the the hypergeometric functions $(6.52,6.53)$, we put in the fieldtheoretic form,

$$
\begin{aligned}
& f(x)=G(x) f(0), \\
& G(x)=<\psi(x) \phi(0)>=\frac{\delta^{2} \ln Z}{\delta J(x) \delta I(0)}=(1-x R)^{-1}, \\
& Z=\int d \psi d \phi e^{-S+I \phi+J \psi}=e^{I(1-x R)^{-1} J}, \\
& S=\int \psi(1-x R) \phi=\int \psi(Q-x P) \varphi, \phi=Q \varphi .
\end{aligned}
$$

When we invent interaction terms, we obtain nontrivial HFT. In terms of the fundamental fields, $\psi, \varphi$, we have local field model.

\subsection{Lauricella Hypergeometric functions (LFs)}

For LFs (see, e.g. [Miller, 1977]), we find the following formulas [Makhaldiani, 201]

$$
\begin{aligned}
& F_{A}\left(a ; b_{1}, \ldots, b_{n} ; c_{1}, \ldots, c_{n} ; z_{1}, \ldots, z_{n}\right)=\frac{(a)_{\delta_{1}+\ldots+\delta_{n}}\left(b_{1}\right)_{\delta_{1} \ldots(}\left(b_{n}\right)_{\delta_{n}}}{\left(c_{1}\right)_{\delta_{1}} \ldots\left(c_{n}\right)_{\delta_{n}}} e^{z_{1}+\ldots+z_{n}} \\
& =\frac{(a)_{\delta_{1}+\ldots+\delta_{n}}}{\left(a_{1}\right)_{\delta_{1}} \ldots\left(a_{n}\right)_{\delta_{n}}} F\left(a_{1}, b_{1} ; c_{1} ; z_{1}\right) \ldots F\left(a_{n}, b_{n} ; c_{n} ; z_{n}\right) \\
& =T^{-1}(a) F^{n}=\Sigma_{m \geq 0} \frac{(a)_{m_{1}+\ldots+m_{n}}\left(b_{1}\right)_{m_{1}} \ldots\left(b_{n}\right)_{m_{n}}}{\left(c_{1}\right)_{m_{1}} \ldots\left(c_{n}\right)_{m_{n}}} \frac{z_{1}^{m_{1}}}{m_{1} !} \ldots \frac{z_{n}^{m_{n}}}{m_{n} !},\left|z_{1}\right|+\ldots+\left|z_{n}\right|<1 ; \\
& F_{B}\left(a_{1}, \ldots, a_{n} ; b_{1}, \ldots, b_{n} ; c ; z_{1}, \ldots, z_{n}\right)=\frac{\left(a_{1}\right)_{\delta_{1}} \ldots\left(a_{n}\right)_{\delta_{n}}\left(b_{1}\right)_{\delta_{1}} \ldots\left(b_{n}\right)_{\delta_{n}}}{(c)_{\delta_{1}+\ldots+\delta_{n}}} e^{z_{1}+\ldots+z_{n}} \\
& =\frac{\left(c_{1}\right)_{\delta_{1}} \ldots\left(c_{n}\right)_{\delta_{n}}}{(c)_{\delta_{1}+\ldots+\delta_{n}}} F\left(a_{1}, b_{1} ; c_{1} ; z_{1}\right) \ldots F\left(a_{n}, b_{n} ; c_{n} ; z_{n}\right)=T(c) F^{n} \\
& =\Sigma_{m \geq 0} \frac{\left(a_{1}\right)_{m_{1}} \ldots\left(a_{n}\right)_{m_{n}}\left(b_{1}\right)_{m_{1}} \ldots\left(b_{n}\right)_{m_{n}}}{(c)_{m_{1}+\ldots+m_{n}}} \frac{z_{1}^{m_{1}}}{m_{1} !} \ldots \frac{z_{n}^{m_{n}}}{m_{n} !},\left|z_{1}\right|<1, \ldots,\left|z_{n}\right|<1 \text {; } \\
& F_{C}\left(a ; b ; c_{1}, \ldots, c_{n} ; z_{1}, \ldots, z_{n}\right)=\frac{(a)_{\delta_{1}+\ldots+\delta_{n}}(b)_{\delta_{1}+\ldots+\delta_{n}}}{\left(c_{1}\right)_{\delta_{1}} \ldots\left(c_{n}\right)_{\delta_{n}}} e^{z_{1}+\ldots+z_{n}} \\
& =\frac{(a)_{\delta_{1}+\ldots+\delta_{n}}(b)_{\delta_{1}+\ldots+\delta_{n}}}{\left(a_{1}\right)_{\delta_{1}} \ldots\left(a_{n}\right)_{\delta_{n}}\left(b_{1}\right)_{\delta_{1} \ldots\left(b_{n}\right)_{\delta_{n}}}} F\left(a_{1}, b_{1} ; c_{1} ; z_{1}\right) \ldots F\left(a_{n}, b_{n} ; c_{n} ; z_{n}\right) \\
& =T^{-1}(a) T^{-1}(b) F^{n}=T^{-1}(b) F_{A} \\
& =\Sigma_{m \geq 0} \frac{(a)_{m_{1}+\ldots+m_{n}}(b)_{m_{1}+\ldots+m_{n}}}{\left(c_{1}\right)_{m_{1}} \ldots\left(c_{n}\right)_{m_{n}}} \frac{z_{1}^{m_{1}}}{m_{1} !} \ldots \frac{z_{n}^{m_{n}}}{m_{n} !},\left|z_{1}\right|^{1 / 2}+\ldots+\left|z_{n}\right|^{1 / 2}<1 ; \\
& F_{D}\left(a ; b_{1}, \ldots, b_{n} ; c ; z_{1}, \ldots, z_{n}\right)=\frac{(a)_{\delta_{1}+\ldots+\delta_{n}}\left(b_{1}\right)_{\delta_{1}} \ldots\left(b_{n}\right)_{\delta_{n}}}{(c)_{\delta_{1}+\ldots \delta_{n}}} e^{z_{1}+\ldots+z_{n}} \\
& =\frac{(a)_{\delta_{1}+\ldots+\delta_{n}}\left(c_{1}\right)_{\delta_{1} \ldots\left(c_{n}\right)_{\delta_{n}}}}{\left(a_{1}\right)_{\delta_{1} \ldots\left(a_{n}\right)_{\delta_{n}}(c){ }_{\delta_{1}+\ldots \delta_{n}}}} F\left(a_{1}, b_{1} ; c_{1} ; z_{1}\right) \ldots F\left(a_{n}, b_{n} ; c_{n} ; z_{n}\right) \\
& =T^{-1}(a) T(c) F^{n}=T(c) F_{A}=T^{-1}(a) F_{B} \\
& =\Sigma_{m \geq 0} \frac{(a)_{m_{1}+\ldots+m_{n}}\left(b_{1}\right)_{m_{1}} \ldots\left(b_{n}\right)_{m_{n}}}{\left(c_{1}\right)_{m_{1}} \ldots\left(c_{n}\right)_{m_{n}}} \frac{z_{1}^{m_{1}}}{m_{1} !} \ldots \frac{z_{n}^{m_{n}}}{m_{n} !},\left|z_{1}\right|<1, \ldots,\left|z_{n}\right|<1 \text {. }
\end{aligned}
$$




\subsection{Lomidze $B_{n}$ function (LBn)}

In the paper ([Lomidze, 1994]) the following formula were proposed

$$
\begin{aligned}
& B_{n}\left(r_{0}, r_{1}, \ldots, r_{n}\right)=\operatorname{det}\left[x_{j}^{i-1} \int_{x_{j-1 / x_{j}}}^{1} u^{i-1}(1-u)^{r_{j}-1} \prod_{k=0, k \neq j}^{n}\left(\frac{x_{j} u-x_{k}}{x_{j}-x_{k}}\right)^{r_{k}-1} \operatorname{du}\right] / \operatorname{det}\left[x_{j}^{i-1}\right] \\
& =\frac{\Gamma\left(r_{0}\right) \Gamma\left(r_{1}\right) \ldots \Gamma\left(r_{n}\right)}{\Gamma\left(r_{o}+r_{1}+\ldots+r_{n}\right)}, 0=x_{0}<x_{1}<x_{2}<\ldots<x_{n}, n \geq 1
\end{aligned}
$$

Let us put the formula in the following factorized form

$$
\begin{aligned}
& L B_{n}(x, r) \equiv \operatorname{det}\left[x_{j}^{i-1} \int_{x_{j-1 / x_{j}}}^{1} d u u^{i+r_{0}-2}(1-u)^{r_{j}-1} \prod_{k=1, k \neq j}^{n}\left(\frac{x_{j} u-x_{k}}{x_{j}-x_{k}}\right)^{r_{k}-1}\right] \\
& =\operatorname{det} V_{n}(x) B_{n}(r), V_{n}(x)=\left[x_{j}^{i-1}\right], B_{n}(r)=\frac{\Gamma\left(r_{0}\right) \Gamma\left(r_{1}\right) \ldots \Gamma\left(r_{n}\right)}{\Gamma\left(r_{0}+r_{1}+\ldots+r_{n}\right)}
\end{aligned}
$$

Now, it is enough to proof this formula for general values of $x_{i}$ and particular values of $r_{i}$, e.g., $r_{i}=1$, and for general values of $r_{i}$ and particular values of $x_{i}$, e.g. $x_{i}=p^{i}, 1 \leq i \leq n$. In the case of $r_{i}=1$, right hand side of the formula is equal to the Vandermonde determinant divided by $n$ ! The left hand side is the determinant of the matrix with elements $A_{i j}=x_{j}^{i-1}\left(1-\left(x_{j-1} / x_{j}\right)^{i}\right) / i$

When we calculate determinant of this matrix, from the row $\mathrm{i}$, we factorize $1 / i, 2 \leq i \leq n$ which gives the $1 / n$ ! the rest matrix we calculate transforming the matrix to the form of the Vandermonde matrix.

This is the half way of the proof. Let us take the concrete values of $x_{i}=p^{i}, 1 \leq i \leq n$, where $p$ is positive integer and general complex values for $r_{i}, 0 \leq i \leq n$, and calculate both sides of the equality. For Vandermonde determinant we find for high values of $p$ the following asymptotic

$$
\operatorname{det} V=p^{N}, N=\sum_{k=2}^{n} k(k-1)=\frac{n\left(n^{2}-1\right)}{3}
$$

The matrix elements are

$$
\begin{aligned}
& B_{i j}=x_{j}^{i-1} \int_{x_{j-1 / x_{j}}}^{1} u^{i+r_{0}-2}(1-u)^{r_{j}-1} \prod_{k=1, k \neq j}^{n}\left(\frac{x_{j} u-x_{k}}{x_{j}-x_{k}}\right)^{r_{k}-1} d u \\
& =x_{j}^{i-1} \prod_{1 \leq k<j}\left(\frac{x_{j}}{x_{j}-x_{k}}\right)^{r_{k}-1} \prod_{j<k \leq n}\left(\frac{x_{k}}{x_{k}-x_{j}}\right)^{r_{k}-1} \int_{x_{j-1 / x_{j}}}^{1} u^{i+r_{0}-2}(1-u)^{r_{j}-1} \\
& \cdot \prod_{1 \leq k<j}\left(u-x_{k} / x_{j}\right)^{r_{k}-1} \prod_{j<k \leq n}\left(1-x_{j} / x_{k} u\right)^{r_{k}-1} d u \\
& =p^{(i-1) j} \int_{0}^{1} u^{i+r_{0}-2+\sum_{k=1}^{j-1}\left(r_{k}-1\right)}(1-u)^{r_{j}-1} d u \\
& =p^{(i-1) j} B\left(i+\sum_{k=0}^{j-1}\left(r_{k}-1\right), r_{j}\right)
\end{aligned}
$$

For $n=2$ we have

$$
\begin{aligned}
& B_{11}=\int_{0}^{1} u^{r_{0}-1}(1-u)^{r_{1}-1} d u=\frac{\Gamma\left(r_{0}\right) \Gamma\left(r_{1}\right)}{\Gamma\left(r_{0}+r_{1}\right)}, \\
& B_{22}=p^{2} \int_{0}^{1} u^{r_{0}+r_{1}-1}(1-u)^{r_{2}-1} d u=\frac{\Gamma\left(r_{0}+r_{1}\right) \Gamma\left(r_{2}\right)}{\Gamma\left(r_{0}+r_{1}+r_{2}\right)},
\end{aligned}
$$




$$
L B_{2} / V_{2}=B_{11} B_{22} / p^{2}=\frac{\Gamma\left(r_{0}\right) \Gamma\left(r_{1}\right) \Gamma\left(r_{2}\right)}{\Gamma\left(r_{0}+r_{1}+r_{2}\right)}
$$

For $n=3$,

$$
\begin{aligned}
& B_{11}=\int_{0}^{1} u^{r_{0}-1}(1-u)^{r_{1}-1}=\frac{\Gamma\left(r_{0}\right) \Gamma\left(r_{1}\right)}{\Gamma\left(r_{0}+r_{1}\right)}=B\left(r_{0}, r_{1}\right), \\
& B_{22}=p^{2} \int_{0}^{1} u^{r_{0}+r_{1}-1}(1-u)^{r_{2}-1}=p^{2} \frac{\Gamma\left(r_{0}+r_{1}\right) \Gamma\left(r_{2}\right)}{\Gamma\left(r_{0}+r_{1}+r_{2}\right)}, \\
& B_{33}=p^{6} \int_{0}^{1} u^{r_{0}+r_{1}+r_{2}-1}(1-u)^{r_{3}-1}=p^{6} \frac{\Gamma\left(r_{0}+r_{1}+r_{2}\right) \Gamma\left(r_{3}\right)}{\Gamma\left(r_{0}+r_{1}+r_{2}+r_{3}\right)} \\
& L B_{3} / V_{3}=B_{11} B_{22} B_{33} / p^{8}=\frac{\Gamma\left(r_{0}\right) \Gamma\left(r_{1}\right) \Gamma\left(r_{2}\right) \Gamma\left(r_{3}\right)}{\Gamma\left(r_{0}+r_{1}+r_{2}+r_{3}\right)}
\end{aligned}
$$

Now it is obvious the last step of the proof [Makhaldiani, 201]]

$$
\begin{aligned}
& L B_{n}(x, r)=\operatorname{det} V_{n}(x) B\left(r_{0}, r_{1}\right) \ldots B\left(r_{0}+r_{1}+\ldots+r_{n-1}, r_{n}\right) \\
& =\operatorname{det} V_{n}(x) B_{n}(r) \\
& V_{n}(x)=\left[x_{j}^{i-1}\right], B_{n}(r)=\frac{\Gamma\left(r_{0}\right) \Gamma\left(r_{1}\right) . . \Gamma\left(r_{n}\right)}{\Gamma\left(r_{0}+r_{1}+\ldots+r_{n}\right)}
\end{aligned}
$$

Note that this proof is based on the factorization assumption $(6.58)$. The proof without this assumption given by I.R.Lomidze is given in [Lomidze, Makhaldiani, 2012].

\subsection{2 $B_{n}$-functions}

We define $B_{n}(a)$-functions as

$$
\begin{aligned}
& B_{n}(a)=\int d^{n} x x_{1}^{a_{1}-1} \ldots x_{n}^{a_{n}-1} \delta\left(1-x_{1}-\ldots x_{n}\right) \\
& =\frac{\Gamma\left(a_{1}\right) \ldots \Gamma\left(a_{n}\right)}{\Gamma\left(a_{1}+\ldots a_{n}\right)}
\end{aligned}
$$

and $b_{n}(a)=a_{1} \ldots a_{n} B_{n}(a)$

$$
\begin{aligned}
& b_{n}(a)=\int d^{n} x\left(x_{1}^{a_{1}}\right)^{\prime} \ldots\left(x_{n}^{a_{n}}\right)^{\prime} \delta\left(1-x_{1}-\ldots x_{n}\right) \\
& =\frac{\Gamma\left(1+a_{1}\right) \ldots \Gamma\left(1+a_{n}\right)}{\Gamma\left(a_{1}+\ldots a_{n}\right)}
\end{aligned}
$$

\subsection{String theory applications}

For (symmetrized, 4-tachyon) Veneziano amplitude we have (see, e.g. [Kaku, 2000])

$$
\begin{aligned}
& B_{s}(\alpha, \beta)=B(\alpha, \beta)+B(\beta, \gamma)+B(\gamma, \alpha)=\int_{-\infty}^{\infty} d x|1-x|^{\alpha-1}|x|^{\beta-1}, \\
& \alpha+\beta+\gamma=1
\end{aligned}
$$

For the p-adic Veneziano amplitude we take

$$
B_{p}(\alpha, \beta)=\int_{Q_{p}} d x|1-x|_{p}^{\alpha-1}|x|_{p}^{\beta-1}=\frac{\Gamma_{p}(\alpha) \Gamma_{p}(\beta)}{\Gamma_{p}(\alpha+\beta)}
$$


Now we obtain the N-tachyon amplitude using fractal calculus. We consider the dynamics of particle given by multicomponent generalization of the action (6.36), $\Phi \rightarrow x^{\mu}$.

For the closed trajectory of the particle passing through $N$ points, we have

$$
\begin{aligned}
& A\left(x_{1}, x_{2}, \ldots, x_{N}\right)=\int d t \int d t_{1} \ldots \int d t_{N} \delta\left(t-\Sigma t_{n}\right) \\
& v\left(x_{1}, t_{1} ; x_{2}, t_{2}\right) v\left(x_{2}, t_{2} ; x_{3}, t_{3}\right) \ldots v\left(x_{N}, t_{N} ; x_{1}, t_{1}\right) \\
& =\int d x(t) \Pi\left(\int d t_{n} \delta\left(x^{\mu}\left(t_{n}\right)-x_{n}^{\mu}\right)\right) \exp (-S[x(t)]) \\
& =\int \Pi\left(d k_{n}^{\mu} \chi\left(k_{n} x_{n}\right)\right) \tilde{A}(k),
\end{aligned}
$$

where

$$
\begin{aligned}
& \tilde{A}(k)=\int d x V\left(k_{1}\right) V\left(k_{2}\right) \ldots V\left(k_{N}\right) \exp (-S) \\
& V\left(k_{n}\right)=\int d t \chi\left(-k_{n} x(t)\right)
\end{aligned}
$$

is vertex function.

Motion equation

$$
D^{\alpha} x^{\mu}-i \Sigma k_{n}^{\mu} \delta\left(t-t_{n}\right)=0
$$

in the momentum representation

$$
|u|^{\alpha} \tilde{x}^{\mu}(u)-i \Sigma_{n} k_{n}^{\mu} \chi\left(-u t_{n}\right)=0
$$

have the solution

$$
\tilde{x}^{\mu}(u)=i \Sigma k_{n}^{\mu} \frac{\chi\left(-u t_{n}\right)}{|u|^{\alpha}}, u \neq 0
$$

the constraint

$$
\Sigma_{n} k_{n}=0
$$

and the zero $\bmod \tilde{x}_{n}^{\mu}(0)$, which is arbitrary. Integration in (6.68) with respect to this zero mod gives the constraint (6.73)). On the solution of the equation (6.70)

$$
x^{\mu}(t)=i D_{t}^{-\alpha} \Sigma_{n} k_{n}^{\mu} \delta\left(t-t_{n}\right)=\frac{i}{\Gamma(\alpha)} \Sigma_{n} k_{n}^{\mu}\left|t-t_{n}\right|^{\alpha-1},
$$

the action (6.36) takes value

$$
\begin{aligned}
& S=-\frac{1}{\Gamma(\alpha)} \Sigma_{n<m} k_{n} k_{m}\left|t_{n}-t_{m}\right|^{\alpha-1}, \\
& \tilde{A}(k)=\int \Pi_{n=1}^{N} d t_{n} \exp (-S)
\end{aligned}
$$

In the limit, $\alpha \rightarrow 1$, for $p$-adic case we obtain

$$
x^{\mu}(t)=-i \frac{p-1}{p \ln p} \Sigma_{n} k_{n}^{\mu} \ln \left|t-t_{n}\right|,
$$




$$
\begin{aligned}
& S[x(t)]=\frac{p-1}{p \ln p} \Sigma_{n<m} k_{n} k_{m} \ln \left|t_{n}-t_{m}\right|, \\
& \tilde{A}(k)=\int \Pi_{n=1}^{N} d t_{n} \Pi_{n<m}\left|t_{n}-t_{m}\right|^{\frac{p-1}{p \ln } k_{n} k_{m}} .
\end{aligned}
$$

Now in the limit $p=q^{-1} \rightarrow 1$ we obtain the proper expressions of the real case

$$
\begin{aligned}
& x^{\mu}(t)=-i \Sigma_{n} k_{n}^{\mu} \ln \left|t-t_{n}\right|, \\
& S[x(t)]=\sum_{n<m} k_{n} k_{m} \ln \left|t_{n}-t_{m}\right|, \\
& \tilde{A}(k)=\int \Pi_{n=1}^{N} d t_{n} \Pi_{n<m}\left|t_{n}-t_{m}\right|_{n} k_{m} .
\end{aligned}
$$

By fractal calculus and vector generalization of the model (6.36), fundamental string amplitudes were obtained in [Makhaldiani, 1988].

\section{Supersymmetric and Adelic mechanisms of Taming of the Cosmological constant problem}

The cosmological constant problem is one of the most serious paradoxes in modern particle physics and cosmology [Weinberg, 1989]. Some astronomical observations indicate that the cosmological constant is many orders of magnitude smaller than estimated in modern theoretical elementary particles physics.

In his attempt, [Einstein, 1917] to apply the general relativity to the whole universe, A. Einstein invented a new term involving a free parameter $\lambda$, the cosmological constant (CC),

$$
R_{\mu v}-\frac{1}{2} R g_{\mu v}=\lambda g_{\mu v}-8 \pi G T_{\mu \nu} .
$$

With this modification he finds a static solution for the universe filled with dust of zero pressure and mass density

$$
\rho=\frac{\lambda}{8 \pi G}
$$

The geometry of the universe was that of a sphere $S_{3}$ with proper circumference $2 \pi r$, where

$$
r=\lambda^{-1 / 2}
$$

so the mass of the universe was

$$
\begin{gathered}
M=2 \pi^{2} r^{3} \rho=\frac{\pi}{4} G^{-1} \lambda^{-1 / 2} \\
\sim r(? !) .
\end{gathered}
$$

It is interesting to describe hadrons with a similar geometric picture corresponding to the low energy QCD.

Any contributions to the energy density of the vacuum acts just like CC. By Lorentz invariance, in the vacuum,

$$
<T_{\mu \nu}>=-<\rho>g_{\mu \nu}
$$


so

$$
\lambda_{e f f}=\lambda+8 \pi G<\rho>
$$

or the total vacuum energy density

$$
\rho_{V}=<\rho>+\frac{\lambda}{8 \pi G}=\frac{\lambda_{e f f}}{8 \pi G} .
$$

The experimental upper bound on $\lambda_{e f f}$ or $\rho_{V}$ is provided by measurements of cosmological redshifts as a function of distance. From the present expansion rate of the universes

$$
\frac{d \ln R}{d t} \equiv H_{0}=100 h \frac{k m}{\sec M p c}, h=0.7 \pm 0.07
$$

we have

$$
H_{0}^{-1}=(1 \div 2) \times 10^{10} y e,\left|\lambda_{e f f}\right| \leq H_{0}^{2},\left|\rho_{V}\right| \leq 10^{-29} \mathrm{~g} / \mathrm{cm}^{2} \simeq 10^{-47} \mathrm{GeV}^{4}
$$

The quantum oscillator with hamiltonian

$$
H=\frac{1}{2} P^{2}+\frac{1}{2} \omega^{2} x^{2}
$$

has the energy spectrum

$$
E_{n}=\hbar \omega(n+1 / 2), n=0,1,2, \ldots
$$

with the lowest, vacuum, value $E_{0}=\hbar \omega / 2$. Normal modes of a quantum field of mass $\mathrm{m}$ are oscillators with frequencies $\omega(k)=\sqrt{k^{2}+m^{2}}$. Summing the zero-point energies of all normal modes of the field up to a wave number cut-off $\Lambda>>m$ yields a vacuum energy density

$$
<\rho>=\int_{0}^{\Lambda} \frac{4 \pi k^{2} d k}{(2 \pi)^{3}} \frac{1}{2} \sqrt{k^{2}+m^{2}} \simeq \frac{\Lambda^{4}}{16 \pi^{2}} .
$$

If we take $\Lambda=(8 \pi G)^{-1 / 2}$, then

$$
<\rho>\simeq 2^{-10} \pi^{-4} G^{-2}=2 \times 10^{71} \mathrm{GeV}^{4} .
$$

We saw that

$$
\left|<\rho>+\frac{\lambda}{8 \pi G}\right| \leq 10^{-47} \mathrm{GeV}^{4} \simeq\left(10^{-3} e V\right)^{4}
$$

so the two terms must cancel to better than 100 decimal places! If we take $\Lambda_{Q C D},\langle\rho\rangle \simeq$ $10^{-6} \mathrm{GeV}^{4} \simeq(300 \mathrm{MeV})^{4}$, the two terms must cancel better to than 40 decimal places. Since the cosmological upper bound on $\left\langle\rho_{\text {eff }}\right\rangle$ is vastly less than any value expected from particle theory, theorists assumed that (for some unknown reason) this quantity is zero. 


\subsection{Supersymmetric mechanism of solution to the CC problem}

A minimal realization of the algebra of supersymmetry

$$
\begin{aligned}
& \left\{Q, Q^{+}\right\}=H, \\
& \{Q, Q\}=\left\{Q^{+}, Q^{+}\right\}=0,
\end{aligned}
$$

is given by a point particle in one dimension, [Witten, 1998]

$$
\begin{aligned}
& Q=a(-i P+W), \\
& Q^{+}=a^{+}(i P+W),
\end{aligned}
$$

where $P=-i \partial / \partial x$, the superpotential $W(x)$ is any function of $\mathrm{x}$, and spinor operators $a$ and $a^{+}$ obey the anticommuting relations

$$
\begin{aligned}
& \left\{a, a^{+}\right\}=1, \\
& a^{2}=\left(a^{+}\right)^{2}=0 .
\end{aligned}
$$

There is a following representation of operators $a, a^{+}$and $\sigma$ by the Pauli spin matrices

$$
\begin{gathered}
a=\frac{\sigma_{1}-i \sigma_{2}}{2}=\left(\begin{array}{ll}
0 & 0 \\
1 & 0
\end{array}\right), \\
a^{+}=\frac{\sigma_{1}+i \sigma_{2}}{2}=\left(\begin{array}{ll}
0 & 1 \\
0 & 0
\end{array}\right), \\
\sigma=\sigma_{3}=\left(\begin{array}{cc}
1 & 0 \\
0 & -1
\end{array}\right) .
\end{gathered}
$$

From formulae (प.15) and (प.16) then we have

$$
H=P^{2}+W^{2}+\sigma W_{x}
$$

The simplest nontrivial case of the superpotential $W=\omega x$ corresponds to the supersimmetric oscillator with Hamiltonian

$$
H=H_{B}+H_{F}, H_{B}=P^{2}+\omega^{2} x^{2}, H_{F}=\omega \sigma
$$

wave function

$$
\psi=\psi_{B} \psi_{F}
$$

and spectrum

$$
\begin{aligned}
& H_{B} \psi_{B n}=\omega(2 n+1) \psi_{B n}, \\
H_{F} \psi_{+}= & \omega \psi_{+}, H_{F} \psi_{-}=-\omega \psi_{-} .
\end{aligned}
$$

The ground state energies of the bosonic and fermionic parts are

$$
E_{B 0}=\omega, E_{F 0}=-\omega,
$$


so the vacuum energy of the supersymmetric oscillator is

$$
<0|H| 0>=E_{0}=E_{B 0}+E_{F 0}=0, \mid 0>=\psi_{B 0} \psi_{F 0} .
$$

Note that the spectrum of this supersymmetric oscillator coincides with the natural numbers, $\mathrm{n}=0,1,2, \ldots$ If you like, we can say that this is number-theoretic motivation of the sypersymmetric models.

Let us see on this toy - solution of the CC problem from the quantum statistical viewpoint. The statistical sum of the supersymmetric oscillator is

$$
Z(\beta)=Z_{B} Z_{F}
$$

where

$$
\begin{array}{r}
Z_{B}=\sum_{n} e^{-\beta E_{B n}}=e^{-\beta \omega}+e^{-\beta \omega(2+1)}+\ldots \\
Z_{F}=\sum_{n} e^{-\beta E_{F n}}=e^{\beta \omega}+e^{-\beta \omega} .
\end{array}
$$

In the low temperature limit,

$$
Z(\beta)=1+O\left(e^{-\beta 2 \omega}\right) \rightarrow 1, \quad \beta=T^{-1},
$$

so CC

$$
\lambda \sim \ln Z \rightarrow 0
$$

In the case of the adelic solution to the $\mathrm{CC}$ problem we will have,

$$
\begin{gathered}
Z(\beta)=\prod_{p \geq 1} Z_{p}=Z_{1} Z_{2} Z_{3} Z_{5} \ldots \\
Z_{1} \equiv Z_{B}, Z_{F} \div Z_{2} Z_{3} Z_{5} \ldots(? !)
\end{gathered}
$$

\subsection{Dyons and Two TeV Unification}

In nonrelativistic approximation the force between two dyons with electric and magnetic charges $\mathfrak{g}_{n}=\left(e_{n}, g_{n}\right), n=1,2$ is

$$
F=\frac{\mathfrak{g}_{1} \mathfrak{g}_{2} \mathbf{r}+\mathfrak{g}_{1} \times \mathfrak{g}_{2} \mathbf{v} \times \mathbf{r}}{4 \pi r^{3}}
$$

where $\mathfrak{g}_{1} \mathfrak{g}_{2}=e_{1} e_{2}+g_{1} g_{2}, \mathfrak{g}_{1} \times \mathfrak{g}_{2}=e_{1} g_{2}-e_{2} g_{1}, \mathbf{r}=\mathbf{r}_{2}-\mathbf{r}_{1}, \mathbf{v}=\mathbf{v}_{2}-\mathbf{v}_{1}, c=1$. Note that, this force depends on the invariant dual combinations of charges: the combination

$$
\left(e_{1}-i g_{1}\right)\left(e_{2}+i g_{2}\right)=e_{1} e_{2}+g_{1} g_{2}+i\left(e_{1} g_{2}-e_{2} g_{1}\right)
$$


is invariant with respect to the continual global dual transformations

$$
e^{i \alpha}(e+i g)=e^{\prime}+i g^{\prime}
$$

From Dirac's quantization of charge

$$
e g=2 \pi \hbar c n, n= \pm 1, \pm 2, \ldots,
$$

we have

$$
g=\frac{e}{2 \alpha} \hbar c n
$$

In the natural system of units, $c=\hbar=1$, and $n=1$, elementary magnetic charge has the value

$$
\begin{aligned}
& g=68.5 e, \\
& \alpha_{g}=\frac{g^{2}}{4 \pi}=\left(\frac{1}{2 \alpha}\right)^{2} \alpha=\frac{1}{4 \alpha}=\frac{137}{4}=34.25
\end{aligned}
$$

The mass of the monopole we can estimate if we suppose that the classical radius of the monopole is not more than electron's one

$$
\begin{aligned}
& m_{e}=\frac{\alpha}{e r_{e}}, m_{g}=\frac{\alpha_{g}}{e r_{g}} \\
& r_{g} \leq r_{e} \Rightarrow m_{g} \geq \frac{\alpha_{g}}{\alpha_{e}} m_{e}=\frac{m_{e}}{4 \alpha^{2}}=4692.25 m_{e} \simeq 2398 \mathrm{MeV} \simeq 2.4 \mathrm{TeV}
\end{aligned}
$$

So, the Two-TeV unification takes place at the monopole scale.

\subsection{Unification of the Two Abelian Couplings}

At the critical point we may have unification of the two abelian couplings, weak-electromagnetic and strong-monopole couplings. According to the Dirac quantization rule, for the electron- $e$ and monopole- $g$ charges we have

$$
e g=2 \pi n, n= \pm 1, \pm 2, \ldots
$$

so, at the selfdual, critical, point, we have prediction:

$$
\alpha_{e}=\alpha_{g}=\frac{n}{2}, n=1,2, \ldots
$$

The minimal-fundamental value of the unified coupling constant is $\alpha=1 / 2$.

Schwinger constructed a quantum field theory of magnetic and electric charges which is relativistically invariant in consequence of the charge quantization condition $e g / \hbar c=4 \pi n, n$ integer, [Schwinger, 1966]. This is more restrictive than Dirac's condition, which would also allow halfintegral values.

Now the minimal value at the unification point is 1 . The next value is 2 . These two values of coupling constant are connected as UV and IR fixed points of one monotone RD interval. 


\subsection{Nonperturbative analytic methods}

Generating functional of the correlators

$$
\begin{aligned}
& Z(g, J)=\int d \varphi e^{-S[\varphi, g]+J \cdot \varphi}=\sum_{n \geq 0} g^{n} Z_{n}(J), \\
& Z_{n}(J)=\oint \frac{d g Z(g, J)}{2 \pi i g^{n+1}}=\oint \frac{d \phi}{2 \pi} e^{-S[\varphi, g]-(i \phi+\rho) n+J \cdot \varphi}, g=e^{\rho+i \phi}
\end{aligned}
$$

Higher $\mathrm{n}$ behavior of the expansion can be defined by stationary-point solutions of the following equations

$$
\begin{aligned}
& \frac{\delta S}{\delta \varphi(x)}=J(x) \\
& g \frac{\partial S}{\partial g}=-n
\end{aligned}
$$

In our model field theory

$$
\begin{aligned}
& S=\int d^{D} x\left(\frac{1}{2} \varphi \Delta \varphi+\frac{g}{N} \varphi^{N}\right), \triangle=-\partial^{2}, \\
& \triangle \varphi+g \varphi^{N-1}=J, \\
& \int d^{D} x \varphi^{N}=-\frac{n N}{g}
\end{aligned}
$$

\section{Sum rules}

Much of the hadronic dynamics at low energies is determined by nucleon and meson exchanges while the intermediate energy region is dominated by various resonances. Chiral symmetry is an important physical constraint that governs the meson-nucleon interactions at low energies and unitarity is an essential property at intermediate energies. A natural link between the low and intermediate energy regions is provided by various sum rules.

\subsection{Gerasimov, Drell-Hearn sum rules}

The Gerasimov, Drell-Hearn (GDH) sum rule [Gerasimov, 1965], [Drenl, Hearn, 1966]

$$
\frac{\pi^{2} \alpha}{m^{2} s} k^{2}=\int_{-\infty}^{\infty} d t[\sigma(t, 1+s)-\sigma(t, 1-s)]
$$

relates the anomalous magnetic moment $k$ of a particle with spin $s$ and mass $m$ to the integral of the difference of polarized total photo-absorption cross-sections $\sigma(t, 1 \pm s)$ for total helicity $(1 \pm s)$ and energy $\omega=\omega_{0} e^{t}$. The anomalous magnetic moment is $k=(g-2) s$, i.e., the deviation of the gyromagnetic ratio $g$ from its natural value of 2 for any spin [Ferrara, Porrati, Telegdi, 1992]. In the case of the proton, e.g., $s=1 / 2, k=1.79$

It is interesting also to consider high values of $s$ for high spin nuclei, magnetics, string and extended particle models. Note that, for $s=0$, both sides of the relation is zero.

Let us present the integral from right-hand side as

$$
\int_{-\infty}^{\infty} d t \sum_{n=-s+1}^{s} A(t, n)=\sum_{n=-s+1}^{s} \int_{-\infty}^{\infty} d t A(t, n)=\sum_{n=-s+1}^{s} a(n)
$$




$$
A(t, n)=\sigma(t, 1+n)-\sigma(t, n), a(n)=\int_{-\infty}^{\infty} d t A(t, n)
$$

One of the solutions of the GDH relation considered as an integral equation is

$$
\begin{aligned}
& a(n)=a=\frac{\pi^{2} \alpha}{2 m^{2}}(g-2)^{2}, \\
& b(n+1)=b(n)+a, b(n)=\int_{-\infty}^{\infty} d t \sigma(t, n)
\end{aligned}
$$

\subsection{Adler-Weisberger sum rule}

The Adler-Weisberger sum rule (AWS) [Adler, 1965], [Weisberger, 1966], [Adler, Dashen, 1965], [Treiman, Witten, Jackiw, Zumino, 1985], [Weinberg, 1996] relates integrals over the total cross section to the scattering amplitude calculated at a subthreshold kinematic point

$$
\begin{gathered}
1-g_{A}^{-2}=\frac{2 m_{N}^{2}}{\pi g_{\pi N}^{2}} \int_{0}^{\infty} d t\left[\sigma^{\pi^{+} N}(t)-\sigma^{\pi^{-} N}(t)\right] \\
=\sin ^{2} \theta=(3 / 5)^{2}
\end{gathered}
$$

where $\left.\sigma^{\pi^{ \pm}} N(t)\right)$ are the pion-nucleon cross sections at the energy $\omega=m_{\pi} e^{t}$. For the value of the axial coupling constant $g_{A}=5 / 4=1 / \cos \theta$, on the right-hand side of the AWS, we will have $\sin ^{2} \theta, \sin \theta=3 / 5, \theta=0.6435011087932845 * 180 / P i=36.86989764584403 \simeq 37^{\circ}$

Note that, we can define the pion decay constant from the Goldberger-Treiman relation

$$
f_{\pi}=\frac{g_{A} m_{N}}{g_{\pi N}}
$$

and put the AWS in the form

$$
g_{A}^{2}=1+\frac{2 f_{\pi}^{2}}{\pi} \int_{0}^{\infty} d t\left[\sigma^{\pi^{+} N}(t)-\sigma^{\pi^{-} N}(t)\right]
$$

\subsection{Bjorken Sum Rule}

The Bjorken sum rule [Bjorken,1966] has been of central importance for studying the spin structure of the nucleon. Accounting for finite $Q^{2}$ corrections to the sum rule, it reads:

$$
\begin{aligned}
& \int_{0}^{1}\left(g_{p 1}-g_{n 1}\right) d x=\frac{g_{A}}{6}\left(1-\frac{\alpha_{s}}{\pi}-3.58\left(\frac{\alpha_{s}}{\pi}\right)^{2}-20.21\left(\frac{\alpha_{s}}{\pi}\right)^{3}+\ldots\right) \\
& +\sum_{k \geq 1} \frac{\mu_{k}}{Q^{2 k}}
\end{aligned}
$$

where the $\mu_{k}$ are higher twist terms. We take the following valence quark parametrization

$$
\int_{0}^{1}\left(g_{p 1}-g_{n 1}\right) d x=\frac{g_{A}}{6}\left(1-\frac{\alpha_{V}}{2}\right), \alpha_{V}=\frac{2 \alpha_{s}}{\pi}+\ldots
$$

The Bjorken sum rule is related to a more general sum rule, the generalized Gerasimov-DrellHearn (GDH) sum rule [Gerasimov, 1965], [Drell, Hearn, 1966], [Anselmino,Lffe,Leader, 1989], [1,Osborne,2001]:

$$
\int_{0}^{1}\left(g_{p 1}-g_{n 1}\right) d x=\frac{Q^{2}}{(4 \pi)^{2} \alpha}\left(G D H_{p}\left(Q^{2}\right)-G D H_{n}\left(Q^{2}\right)\right)
$$


Since the generalized GDH sum is, in principle, calculable at any $Q^{2}$, it can be used to study the transition from the partonic to hadronic degrees of freedom of the strong force. The Bjorken sum is the flavor non-singlet part of the GDH sum. This leads to simplifications that may help in linking the $(\chi \mathrm{PT})$ validity domain to the pQCD validity domain [Burkert,200]]. Hence the Bjorken sum appears as a key quantity to study the hadron-parton transition.

\subsection{Renormdynamics of space dimension}

Let us consider the Coulomb problem

$$
\Delta \varphi=e \delta^{D}(x), \varphi(r)=\frac{e r^{(2-D)}}{(2-D) \Omega_{D}}, \Omega_{D}=\frac{2 \pi^{\frac{D}{2}}}{\Gamma\left(\frac{D}{2}\right)}
$$

For corresponding action $S$,

$$
S=\int d^{D} x\left(\frac{1}{2}(\nabla \varphi)^{2}+\rho \varphi\right)=\int d^{D} x \frac{1}{2}(\nabla \varphi)^{2}+e \varphi(0), \rho(x)=e \delta^{D}(x)
$$

For radially symmetric case

$$
S=\int d r r^{D-1} \Omega_{D} \frac{1}{2}\left(\varphi^{\prime}\right)^{2}+e \varphi(0)
$$

the problem reduce to the one dimensional problem and the dimension $D$ enter as external (control) parameter. Motion equation for $\varphi$ is

$$
\varphi^{\prime \prime}+\frac{D-1}{r} \varphi^{\prime}=\rho, \rho=e \delta^{D}(x)
$$

with usual solution

$$
\varphi(r, D)=-\frac{e}{(D-2) \Omega_{D} r^{D-2}}
$$

Note that, for this solution $\varphi(0)$ is divergent. The following regularized version of the point charge problem,

$$
\int d^{D} x \rho(x)=e \Omega_{D} \int d r r^{D-1} \rho(r)=e \int d r \delta\left(r-r_{0}\right)=e, \rho(r)=e \frac{\delta\left(r-r_{0}\right)}{\Omega_{D} r^{D-1}}
$$

is consistent.

Let us calculate the value of action on the solution and the regularized source

$$
\begin{aligned}
& S=\int_{0}^{r_{0}} \Omega_{D} r^{D-1} \frac{1}{2}\left(\varphi^{\prime}\right)^{2}+\int_{r_{0}}^{\infty} \Omega_{D} r^{D-1} \frac{1}{2}\left(\varphi^{\prime}\right)^{2}+e \varphi\left(r_{0}\right) \\
& =\left.\frac{1}{2} \frac{e^{2}}{(2-D) \Omega_{D}} r^{2-D}\right|_{0} ^{r_{0}}+\left.\frac{1}{2} \frac{e^{2}}{(2-D) \Omega_{D}} r^{2-D}\right|_{r_{0}} ^{\infty}+e \varphi\left(r_{0}\right)
\end{aligned}
$$

This expression has finite, or physical, value

$$
S_{e}=\frac{1}{2} \frac{e^{2}}{(2-D) \Omega_{D}} r_{0}^{2-D}
$$


when $D<2, r<r_{0}$ and $D>2, r>r_{0}$. So, we have a good motivation of the evolution of the dimension with $r$. Then we can take the fundamental value for action $S_{e}=\hbar=1$, that restricts the values of $r_{0}$ and $D$, for $r<r_{0}$. Note that, we have extra bonus, the potentials corresponding to $D<2$ are confining.

If we ask optimal property of the action not only with respect of the field $\varphi$, but also with respect to the dimension $D$, we obtain the following system of equations

$$
\begin{aligned}
& \frac{\partial}{\partial D}\left(r^{D-1} \Omega_{D}\right)=0 \Rightarrow r^{D-1} \Omega_{D}(r)=f(r), \\
& \frac{\partial}{\partial r}\left(r^{D-1} \Omega_{D} \frac{\partial}{\partial r} \varphi\right)=r^{D-1} \Omega_{D} \rho(r) \Rightarrow \frac{\partial^{2}}{\partial r^{2}} \varphi+\left(\frac{D-1}{r}+\left(\ln \Omega_{D}\right)_{r}\right) \frac{\partial}{\partial r} \varphi=\rho(r)
\end{aligned}
$$

where we suppose that the function $D(r)$ pass through the point $D(a)=3$. If we insert this constraint into the action, we find

$$
S=4 \pi \int d r a^{D(r)-1} \frac{1}{2}\left(\varphi^{\prime}\right)^{2}+e \varphi(0)
$$

with corresponding motion equation for $\varphi$

$$
\begin{aligned}
& \left(a^{D(r)} \varphi^{\prime}\right)^{\prime}=0 \Rightarrow \varphi(r)=\int a^{-D(r)} d r+\text { const } \\
& S=2 \pi / a \varphi(r)+e \varphi(0)
\end{aligned}
$$

The following regularized version of the point charge problem,

$$
\int d^{D} x \rho(x)=e \Omega_{D} \int d r r^{D-1} \rho(r)=e \int d r \delta\left(r-r_{0}\right)=e
$$

is consistent with the requirement

$$
r^{D-1} \Omega_{D}=1
$$

The standard motion equation and its solution are

$$
\begin{aligned}
& \frac{d}{d r}\left(r^{D-1} \frac{d \varphi}{d r}\right)=r^{D-1} \rho(r), \\
& \varphi(r)=\frac{e r^{(2-D)}}{(D-2) \Omega_{D}}, \rho(x)=e \delta^{D}(x), \Omega_{D} \int d r r^{D-1} \rho(r)=e \int d r \delta\left(r-r_{0}\right)=e
\end{aligned}
$$

If we ask optimal property of the action with respect to the dimension $D$, we find that

$$
x^{D-1} \Omega_{D}=4 \pi, D(1)=4 \pi
$$

where we assume that the function $D(x)$ pass through the point $D(1)=3$. So, it is ease to find radius $x$ as a function of the dimension

$$
x(D)=\left(\frac{\Omega_{D}}{4 \pi}\right)^{\frac{1}{1-D}}=\left(\frac{4 \pi}{\Omega_{D}}\right)^{\frac{1}{D-1}}=\left(\frac{2 \Gamma\left(\frac{D}{2}\right)}{\pi^{\frac{D-2}{2}}}\right)^{\frac{1}{D-1}}, \Omega_{D}=\frac{2 \pi^{\frac{D}{2}}}{\Gamma\left(\frac{D}{2}\right)}, x(3)=1 .
$$

By plot of this function we will see also its inverse function, $D(r)$. 


\subsection{Feynman integrals}

The problem of evaluating Lorentz-covariant Feynman integrals over loop momenta originated in the early days of perturbative quantum field theory. When quantum-field amplitudes are constructed within perturbation theory, multiloop Feynman integrals appear. They are integrals over so-called loop momenta. Feynman has invented their graph-theoretical interpretation. Feynman integrals are usually complicated objects even in a one-loop approximation, so that the number of loops equal to two is already considered big.

In Feynman representation, for a product of two propagators raised to general powers, one writes down the following relation:

$$
\begin{aligned}
& \left(m_{1}^{2}-p_{1}^{2}\right)^{-a_{1}}\left(m_{2}^{2}-p_{2}^{2}\right)^{-a_{2}}=\frac{\Gamma\left(a_{1}+a_{2}\right)}{\Gamma\left(a_{1}\right) \Gamma\left(a_{2}\right)} \int_{0}^{1} \frac{d x_{1} d x_{2} x_{1}^{a_{1}-1} x_{2}^{a_{2}-1} \delta\left(x_{1}+x_{2}-1\right)}{\left(x_{1}\left(m_{1}^{2}-p_{1}^{2}\right)+x_{2}\left(m_{2}^{2}-p_{2}^{2}\right)\right)^{a_{1}+a_{2}}} \\
& =\frac{\Gamma\left(a_{1}+a_{2}\right)}{\Gamma\left(a_{1}\right) \Gamma\left(a_{2}\right)} \int_{0}^{1} d x_{1} d x_{2} x_{1}^{a_{1}+\delta_{1}-1} x_{2}^{a_{2}+\delta_{2}-1}\left(b_{1}+b_{2}\right)^{-a_{1}-a_{2}} \\
& =\frac{\left(\delta_{1}\right)_{a_{1}}\left(\delta_{2}\right)_{a_{2}}}{\left(\delta_{1}+\delta_{2}\right)_{a_{1}+a_{2}}}\left(b_{1}+b_{2}\right)^{-a_{1}-a_{2}}, b_{n}=m_{n}^{2}-p_{n}^{2}, \delta_{n}=b_{n} \partial_{b_{n}}, n=1,2 \\
& (a)_{b}=\frac{\Gamma(a+b)}{\Gamma(b)}
\end{aligned}
$$

We evaluate $L$-loop Feynman integrals in $D=4-2 e$ dimensions with $N$ internal lines with momenta $q_{n}$ and masses $m_{n}$, and $E$ external legs with momenta $p_{e}$ :

$$
\begin{aligned}
& I_{L}=\int \frac{d^{D} k_{1} \ldots d^{D} k_{L}}{\left(q_{1}^{2}-m_{1}^{2}\right)^{a_{1}} \ldots\left(q_{L}^{2}-m_{L}^{2}\right)^{a_{N}}}=K \int d x_{1} x_{1}^{a_{1}-1} \ldots d x_{N} x_{N}^{a_{N}-1} \delta\left(1-x_{1}-\ldots-x_{N}\right) M^{-a} \\
& q_{n}^{2}=\left(\alpha_{n l} k_{l}-\beta_{n e}\right)^{2}, M=x_{n}\left(q_{n}^{2}-m_{n}^{2}\right)=k A k-2 B k=k_{n} A_{n m} k_{m}-2 k_{n} B_{n} \\
& l=1, \ldots, L, e=1, \ldots, E, a=a_{1}+\ldots+a_{N}
\end{aligned}
$$

\subsection{Dynamics and Renormdynamics}

We have seen that the quantitative values and qualitative content of the given field theory depends on the scale (parameter, e.g. $\mu$-renormalization point, $g=g(\mu), A=A(\mu)$ ). In QCD e.g. the effective action have the following form

$$
S(\mu)=\frac{1}{g^{2}(\mu)} \int d^{D} x \mathscr{L}(A(\mu)),
$$

so variation with respect the change of scale gives

$$
\delta S=-2 \frac{\beta(g)}{g^{3}} S+\frac{1}{g^{2}} \int d^{D} x \frac{\delta \mathscr{L}}{\delta A} \delta A
$$

and the following two statements are equivalent,

$$
\delta S=0, \beta(g)=0 \Leftrightarrow \delta S=0, \frac{\delta \mathscr{L}}{\delta A}=0
$$

So, from renorminvariance of the effective action, $\delta S=0$, follows that at the conformal symmetric points, fixed points of $\mathrm{RD},(\beta(g)=0)$, the motion equations for fields are satisfied. The solutions of the motion equations are selfsimilar, their are generally fractals. In string theory, the connection between conformal invariance of the effective theory on the parametric world sheet and the motion equations of the fields on the embedding space is well known [Green,Schwarz,Witten,1987]. 


\section{Multiparticle Production and QCD Renormdynamics}

It is sixty years since Yang and Mills (1954) performed their pioneering work on gauge theories, and we have in our hands a good candidate for a theory of the strong interactions based, precisely, on a non-Abelian gauge theory, QCD.

We considered the main properties of the renormdynamics, corresponding motion equations and their solutions on the examples of QCD and other field theory models.

According to the high energy limit of QCD, inelastic interactions are dominated by the multiRegge final states. The appropriate gauge-invariant objects, which simultaneously incorporate the transverse momentum degrees of freedom, are Reggeized gluons, quarks and antiquarks.

\subsection{Renormdynamics of Observable Quantities in High Energy Physics}

With the advent of any new hadron accelerator the quantities first studied are charged particle multiplicities. The multiparticle production can be described by the probability distribution $P_{n}$ which is a superposition of some unknown distribution of sources $F$, and the Poisson distribution describing particle emission from one source. This is a typical situation in many microscopic models of multiparticle production.

Independently radiating valence quarks and corresponding negative binomial distribution presents phenomenologically preferable mechanism of hadronization in multiparticle production processes.

\subsection{Multiparticle production}

Let us consider $l$-particle semi-inclusive distribution

$$
\begin{aligned}
& F_{l}(n, q)=\frac{d^{l} \sigma_{n}}{\bar{d} q_{1} \ldots \bar{d} q_{l}}=\frac{1}{n !} \int \prod_{i=1}^{n} \bar{d} q_{i}^{\prime} \delta\left(p_{1}+p_{2}-\Sigma_{i=1}^{l} q_{i}-\Sigma_{i=1}^{n} q_{i}^{\prime}\right) \\
& \left.\cdot \mid M_{n+l+2}\left(p_{1}, p_{2}, q_{1}, \ldots, q_{l}, q_{1}^{\prime}, \ldots, q_{n}^{\prime} ; g(\mu), m(\mu)\right), \mu\right)\left.\right|^{2}, \\
& \bar{d} p \equiv \frac{d^{3} p}{E(p)}, E(p)=\sqrt{p^{2}+m^{2}} .
\end{aligned}
$$

From the renormdynamic equation

$$
D M_{n+l+2}=\frac{\gamma}{2}(n+l+2) M_{n+l+2}
$$

we obtain

$$
\begin{aligned}
& D F_{l}(n, q)=\gamma(n+l+2) F_{l}(n, q), \\
& D F_{l}(q)=\gamma(<n>+l+2) F_{l}(q), \\
& D<n^{k}(q)>=\gamma\left(<n^{k+1}(q)>-<n^{k}(q)><n(q)>\right), \\
& D C_{k}=\gamma<n(q)>\left(C_{k+1}-C_{k}\left(1+k\left(C_{2}-1\right)\right)\right) \\
& F_{l}(q) \equiv \frac{d^{l} \sigma}{\bar{d} q_{1} \ldots \bar{d} q_{l}}=\sum_{n} \frac{d^{l} \sigma_{n}}{\bar{d} q_{1} \ldots \bar{d} q_{l}},<n^{k}(q)>=\frac{\sum_{n} n^{k} d^{l} \sigma_{n} / \bar{d} q^{l}}{\sum_{n} d^{l} \sigma_{n} / \bar{d} q^{l}} \\
& C_{k}=\frac{<n^{k}(q)>}{<n(q)>^{k}}
\end{aligned}
$$




\subsection{Scaling relations for multi particle cross sections}

From dimensional considerations, the following combination of cross sections [Koba et al, 1972] must be universal function

$$
<n>\frac{\sigma_{n}}{\sigma}=\Psi\left(\frac{n}{<n>}\right) .
$$

Corresponding relation for the inclusive cross sections is [Matveev et al, 1976].

$$
<n(p)>\frac{d \sigma_{n}}{\bar{d} p} / \frac{d \sigma}{\bar{d} p}=\Psi\left(\frac{n}{<n(p)>}\right) .
$$

Indeed, let us define $n$-dimension of observables [Makhaldiani, 1980]

$$
[n]=1,\left[\sigma_{n}\right]=-1, \sigma=\Sigma_{n} \sigma_{n},[\sigma]=0,[<n>]=1 .
$$

The following expression does not depend on any dimensional quantities and must have a corresponding universal form

$$
P_{n}=<n>\frac{\sigma_{n}}{\sigma}=\Psi\left(\frac{n}{<n>}\right) .
$$

For any discrete variable $n$, if the change of summation on the integration is good approximation, we can invent corresponding dimension and use dimensional counting.

Let us find an explicit form of the universal functions using renormdynamic equations. From the definition of the moments we have

$$
C_{k}=\int_{0}^{\infty} d x x^{k} \Psi(x)
$$

so they are universal parameters,

$$
\begin{aligned}
& D C_{k}=0 \Rightarrow C_{k+1}=\left(1+k\left(C_{2}-1\right)\right) C_{k} \Rightarrow \\
& C_{k}=\left(1+(k-1)\left(C_{2}-1\right)\right) \ldots\left(1+2\left(C_{2}-1\right)\right) C_{2} .
\end{aligned}
$$

Now we can invert momentum transform and find (see [Makhaldiani, 1980]) universal functions [Ernst, Schmit, 1976], [Darbaidze et al, 1978].

$$
\begin{aligned}
& \Psi(z)=\frac{1}{2 \pi i} \int_{-i \infty}^{+i \infty} d n z^{-n-1} C_{n}=\frac{c^{c}}{\Gamma(c)} z^{c-1} e^{-c z}, \\
& C_{2}=1+\frac{1}{c}
\end{aligned}
$$

The value of the parameter $c$ can be measured from the dispersion low,

$$
\begin{aligned}
& D=\sqrt{\left\langle n^{2}>-<n\right\rangle^{2}}=\sqrt{C_{2}-1}<n>=A<n>, \\
& A=\frac{1}{\sqrt{c}} \simeq 0.6, c=2.8 ; \\
& (c=3, A=0.58)
\end{aligned}
$$

which is in accordance with $n$-dimension counting. 


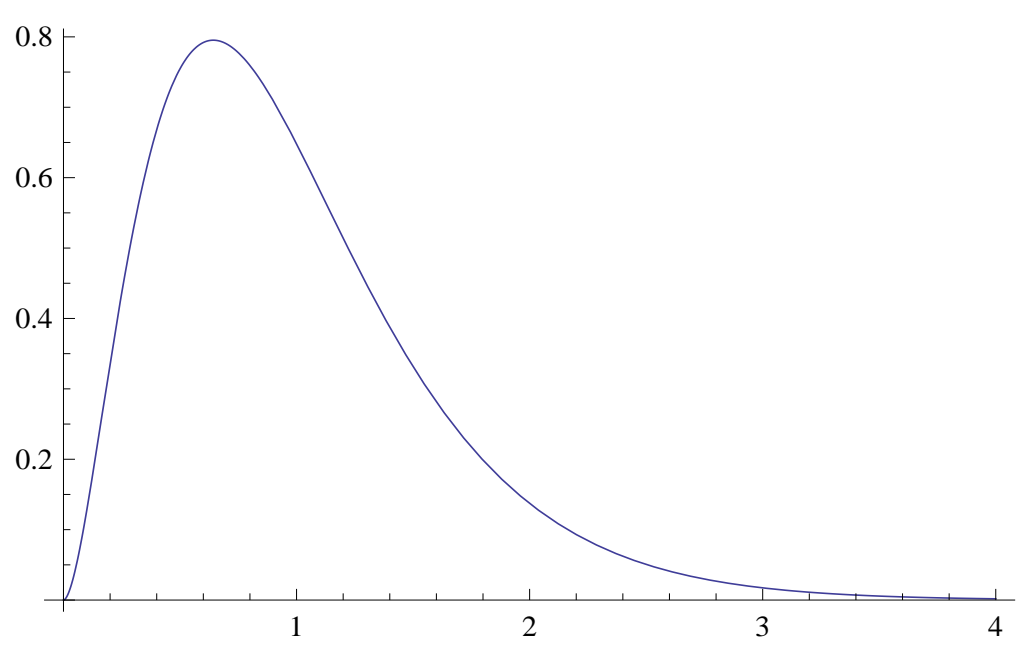

Figure 1: KNO distribution, $\Psi(z)$, with $c=2.8$

\section{4 $1 /<n>$ correction to the scaling function}

We can calculate also $1 /<n>$ correction to the scaling function

$$
\begin{aligned}
& <n>\frac{\sigma_{n}}{\sigma}=\Psi=\Psi_{0}\left(\frac{n}{<n>}\right)+\frac{1}{<n>} \Psi_{1}\left(\frac{n}{<n>}\right), \\
& C_{k}=C_{k}^{0}+\frac{1}{<n>} C_{k}^{1}, \\
& C_{k}^{0}=\int_{0}^{\infty} d x x^{k} \Psi_{0}(x), C_{k}^{1}=\int_{0}^{\infty} d x x^{k} \Psi_{1}(x), \\
& \Psi_{1}(z)=\frac{1}{2 \pi i} \int_{-i \infty}^{+i \infty} d n z^{-n-1} C_{n}^{1}=\frac{C_{2}^{1} c^{2}}{2}\left(z-2+\frac{c-1}{c z}\right) \Psi_{0}
\end{aligned}
$$

\subsection{Characteristic function for KNO}

The characteristic function we define as

$$
\Phi(t)=\int_{0}^{\infty} d x e^{t x} \Psi(x)=(1-t / c)^{-c}, \operatorname{Re}(t)<c
$$

For the moments of the distribution, we have

$$
\Phi^{(k)}(0)=C_{k}=(-c)(-c-1) \ldots(-c-k+1)(-1 / c)^{k}=\frac{\Gamma(c+k)}{\Gamma(c) c^{k}}
$$

Note that it is an infinitely divisible characteristic function, i.e.

$$
\Phi(t)=\left(\Phi_{n}(t)\right)^{n}, \Phi_{n}(t)=(1-t / c)^{-c / n}
$$

If we calculate observable(mean) value of $x$, we find

$$
\begin{aligned}
& <x>=\Phi^{\prime}(0)=n \Phi(0)_{n}{ }^{\prime}=n<x>_{n}, \\
& <x>_{n}=\frac{<x>}{n}
\end{aligned}
$$

For the second moment and dispersion, we have

$$
<x^{2}>=\Phi^{(2)}(0)=n<x^{2}>_{n}+n(n-1)<x>_{n}^{2},
$$




$$
\begin{aligned}
& \left.\mathrm{D}^{2}=<x^{2}>-<x\right\rangle^{2}=n\left(<x^{2}>_{n}-<x>_{n}^{2}\right)=n \mathrm{D}_{n}^{2} \\
& \mathrm{D}_{n}^{2}=\frac{\mathrm{D}^{2}}{n}=\frac{\mathrm{D}^{2}}{<x>}<x>_{n}
\end{aligned}
$$

In a sense, any Hamiltonian quantum (and classical) system can be described by infinitely divisible distributions, because in the functional integral formulation, we use the following step

$$
U(t)=e^{-i t H}=\left(e^{-i \frac{t}{N} H}\right)^{N}
$$

In the case of scalar field theory

$$
\begin{aligned}
& L(\varphi)=\frac{1}{2} \partial_{\mu} \varphi \partial^{\mu} \varphi-\frac{m^{2}}{2} \varphi^{2}-\frac{g}{n} \varphi^{n} \\
& =g^{\frac{2}{2-n}}\left(\frac{1}{2} \partial_{\mu} \phi \partial^{\mu} \phi-\frac{m^{2}}{2} \phi^{2}-\frac{1}{n} \phi^{n}\right)
\end{aligned}
$$

so, to the constituent field $\phi_{N}$ corresponds higher value of the coupling constant,

$$
g_{N}=g N^{\frac{n-2}{2}}
$$

For weak nonlinearity, $n=2+2 \varepsilon, d=2 / \varepsilon+2, g_{N}=g\left(1+\varepsilon \ln N+O\left(\varepsilon^{2}\right)\right)$

\section{Negative binomial distribution}

Negative binomial distribution (NBD) is defined as

$$
P(n)=\frac{\Gamma(n+r)}{n ! \Gamma(r)} p^{n}(1-p)^{r}, \sum_{n \geq 0} P(n)=1,
$$

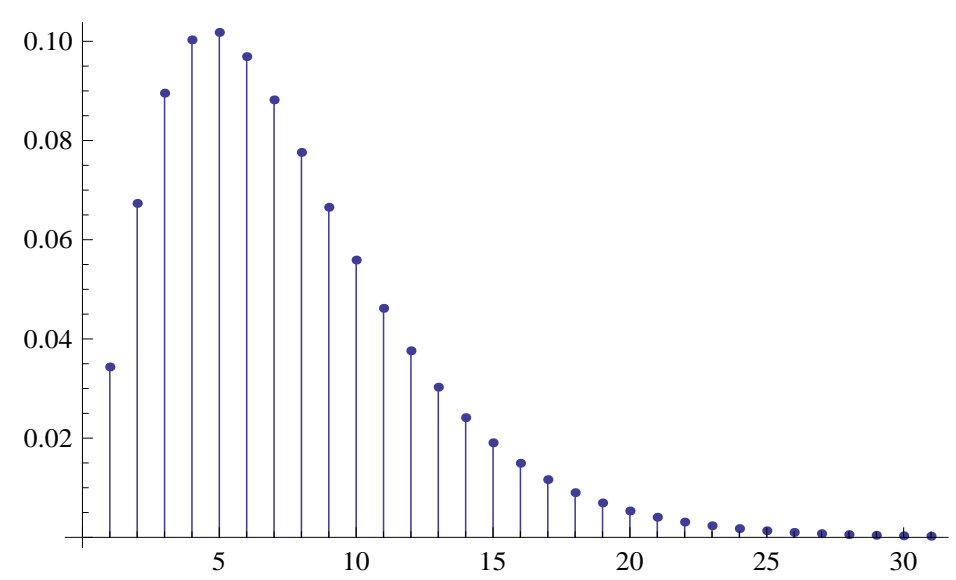

Figure 2: $P(n), r=2.8, p=0.3,<n>=6$

NBD provides a very good parametrization for multiplicity distributions in $e^{+} e^{-}$annihilation; in deep inelastic lepton scattering; in proton-proton collisions; in proton-nucleus scattering.

Hadronic collisions at high energies (LHC) lead to charged multiplicity distributions whose shapes are well fitted by a single NBD in fixed intervals of central (pseudo)rapidity $\eta$ [ALICE, 2010].

It is interesting to understand how NBD fits such a different reactions? 


\subsection{NBD and KNO scaling}

Let us consider NBD for normed topological cross sections

$$
\begin{aligned}
& \frac{\sigma_{n}}{\sigma}=P(n)=\frac{\Gamma(n+k)}{\Gamma(n+1) \Gamma(k)}\left(\frac{k}{<n>}\right)^{k}\left(1+\frac{k}{<n>}\right)^{-(n+k)} \\
& =\frac{\Gamma(n+k)}{\Gamma(n+1) \Gamma(k)}\left(1+\frac{k}{<n>}\right)^{-n}\left(1+\frac{<n>}{k}\right)^{-k} \\
& =\frac{\Gamma(n+k)}{\Gamma(n+1) \Gamma(k)}\left(\frac{<n>}{<n>+k}\right)^{n}\left(\frac{k}{k+<n>}\right)^{k}, \\
& =\frac{\Gamma(k+n)}{\Gamma(k) n !} \frac{\left(\frac{k}{<n>}\right)^{k}}{\left(1+\frac{k}{<n>}\right)^{k+n}}, \\
& r=k>0, p=\frac{<n>}{<n>+k} .
\end{aligned}
$$

The generating function for NBD is

$$
\begin{aligned}
& \left.F(h)=\left(1+\frac{<n>}{k}(1-h)\right)^{-k}=\left(1+\frac{<n>}{k}\right)^{-k}(1-a h)\right)^{-k}, \\
& a=p=\frac{<n>}{<n>+k} .
\end{aligned}
$$

Indeed,

$$
\begin{aligned}
& (1-a h))^{-k}=\frac{1}{\Gamma(k)} \int_{0}^{\infty} d t t^{k-1} e^{-t(1-a h)} \\
& =\frac{1}{\Gamma(k)} \int_{0}^{\infty} d t t^{k-1} e^{-t} \sum_{0}^{\infty} \frac{(t a h)^{n}}{n !} \\
& =\sum_{0}^{\infty} \frac{\Gamma(n+k) a^{n}}{\Gamma(k) n !} h^{n}, \\
& P(n)=\left(1+\frac{<n>}{k}\right)^{-k} \frac{\Gamma(n+k)}{\Gamma(k) n !}\left(\frac{<n>}{<n>+k}\right)^{n} \\
& =\frac{k^{k} \Gamma(n+k)}{\Gamma(k) \Gamma(n+1)}(<n>+k)^{-(n+k)}<n>^{n} \\
& =\frac{\Gamma(n+k)}{\Gamma(n+1) \Gamma(k)}\left(\frac{k}{<n>}\right)^{k}\left(1+\frac{k}{<n>}\right)^{-(n+k)}
\end{aligned}
$$

The Bose-Einstein distribution is a special case of NBD with $k=1$.

If $k$ is negative, the NBD becomes a positive binomial distribution, narrower than Poisson (corresponding to negative correlations).

For negative (integer) values of $k=-N$, we have Binomial GF

$$
\begin{aligned}
& F_{b d}=\left(1+\frac{<n>}{N}(h-1)\right)^{N}=(a+b h)^{N}, a=1-\frac{<n>}{N}, b=\frac{<n>}{N}, \\
& P_{b d}(n)=C_{N}^{n}\left(\frac{<n>}{N}\right)^{n}\left(1-\frac{<n>}{N}\right)^{N-n}
\end{aligned}
$$

(In a sense) we have a (quantum) spectrum for the parameter $k$, which contains any (positive) real values and (with finite number of states) the negative integer values, $(0 \leq n \leq N)$

From the generating function we have

$$
<n^{2}>=\left.\left(\frac{h d}{d h}\right)^{2} F(h)\right|_{h=1}=\frac{k+1}{k}<n>^{2}+<n>,
$$


for dispersion we obtain

$$
\begin{aligned}
& D=\sqrt{<n^{2}>-<n>^{2}}=\frac{1}{\sqrt{k}}<n>\left(1+\frac{k}{<n>}\right)^{1 / 2} \\
& =\frac{1}{\sqrt{k}}<n>+\frac{\sqrt{k}}{2}+O(1 /<n>),
\end{aligned}
$$

So, the dispersion low for KNO and NBD distributions are the same, with $c=k$, for high values of the mean multiplicity.

The factorial moments of NBD,

$$
F_{m}=\left.\left(\frac{d}{d h}\right)^{m} F(h)\right|_{h=1}=\frac{<n(n-1) \ldots(n-m+1)>}{<n>^{m}}=\frac{\Gamma(m+k)}{\Gamma(m) k^{m}},
$$

and usual normalized moments of KNO (Q.14) coincides.

\subsection{Fractal factorial and cumulant moments}

Using fractal calculus (see e.g. [Makhaldiani, 2003]),

$$
\begin{aligned}
D_{0, x}^{-\alpha} f & =\frac{|x|^{\alpha}}{\Gamma(\alpha)} \int_{0}^{1}|1-t|^{\alpha-1} f(x t) d t,=\frac{|x|^{\alpha}}{\Gamma(\alpha)} B(\alpha, \partial x) f(x) \\
& =|x|^{\alpha} \frac{\Gamma(\partial x)}{\Gamma(\alpha+\partial x)} f(x), f(x t)=t^{x \frac{d}{d x}} f(x) .
\end{aligned}
$$

we can define factorial and cumulant moments for any complex indexes,

$$
\begin{aligned}
& F_{-q}=<n>\left.^{q} D_{0, x}^{-q} G_{N B D}(x)\right|_{x=0}=\frac{k^{q} \Gamma(k-q)}{\Gamma(k)}, \\
& K_{-q}=<n>\left.^{q} D_{0, x}^{-q} \ln G_{N B D}(x)\right|_{x=0}=k^{q+1} \Gamma(-q), \\
& H_{-q}=\frac{\Gamma(k+1) \Gamma(-q)}{\Gamma(k-q)}
\end{aligned}
$$

\subsection{The KNO as asymptotic NBD}

Let us show that NBD is a discrete distribution corresponding to the KNO scaling,

$$
\lim _{<n>\rightarrow \infty}<n>\left.P_{n}\right|_{\frac{n}{<n>}=z}=\Psi(z)
$$

Indeed, using the following asymptotic formula

$$
\Gamma(x+1)=x^{x} e^{-x} \sqrt{2 \pi x}\left(1+\frac{1}{12 x}+O\left(x^{-2}\right)\right),
$$

we find

$$
\begin{aligned}
& <n>P_{n}=<n>\frac{(n+k-1)^{n+k-1} e^{-(n+k-1)}}{\Gamma(k) n^{n} e^{-n}} \frac{k^{k}}{n^{k}}<n>z^{k} e^{-k \frac{n+k}{<n>}} \\
& =\frac{k^{k}}{\Gamma(k)} z^{k-1} e^{-k z}+O(1 /<n>)
\end{aligned}
$$


We can calculate also $1 /<n>$ correction term to the KNO from the NBD. The answer is

$$
\Psi=\frac{k^{k}}{\Gamma(k)} z^{k-1} e^{-k z}\left(1+\frac{k^{2}}{2}\left(z-2+\frac{k-1}{k z}\right) \frac{1}{<n>}\right)
$$

This form coincides with the corrected KNO (QD2) for $c=k$ and $C_{2}^{1}=1$.

We have seen that KNO characteristic function (2.13) and NBD GF ([0.3]) have almost same form. This relation become in coincidence if

$$
c=k, t=(h-1) \frac{<n>}{k}
$$

Now the definition of the characteristic function (2.13]) can be read as

$$
\int_{0}^{\infty} e^{-<n>z(1-h)} \Psi(z) d z=\left(1+\frac{<n>}{k}(1-h)\right)^{-k}
$$

which means that Poisson GF weighted by KNO distribution gives NBD GF. Because of this, the NBD is the gamma-Poisson (mixture) distribution. This is the exact and universal picture of hadronization in multiparticle production processes.

\subsection{NBD, Poisson and Gauss distributions}

Fore high values of $x_{2}=k$ the NBD distribution reduces to the Poisson distribution

$$
\begin{aligned}
& F\left(x_{1}, x_{2}, h\right)=\left(1+\frac{x_{1}}{x_{2}}(1-h)\right)^{-x_{2}} \Rightarrow e^{-x_{1}(1-h)}=e^{-<n>} e^{h<n>} \\
& =\sum P(n) h^{n}, \\
& P(n)=e^{-<n>} \frac{<n>^{n}}{n !}
\end{aligned}
$$

For the Poisson distribution

$$
\begin{aligned}
& \left.\frac{d^{2} F(h)}{d h^{2}}\right|_{h=1}=<n(n-1)>=<n>^{2}, \\
& D^{2}=<n^{2}>-<n>^{2}=<n>.
\end{aligned}
$$

In the case of NBD, we had the following dispersion low

$$
D^{2}=\frac{1}{k}<n>^{2}+<n>
$$

which coincides with the previous expression for high values of $k$. Poisson GF belongs to the class of the infinitely divisible distributions,

$$
F(h,<n>)=(F(h,<n>/ k))^{k}
$$

For high values of $\langle n>$, the Poisson distribution reduces to the Gauss distribution

$$
P(n)=e^{-<n>} \frac{<n>^{n}}{n !} \Rightarrow \frac{1}{\sqrt{2 \pi<n>}} \exp \left(-\frac{(n-<n>)^{2}}{2<n>}\right)
$$

For high values of $k$ in the integral relation (10.16), in the KNO function dominates the value $z_{c}=1$ and both sides of the relation reduce to the Poisson GF. 


\subsection{Multiplicative properties of KNO and NBD and corresponding motion equations}

A Bose-Einstein, or geometrical, distribution is a thermal distribution for single state systems. An useful property of the negative binomial distribution with parameters

$$
<n>, k
$$

is that it is (also) the distribution of a sum of $k$ independent random variables drawn from a BoseEinstein distribution with mean $\langle n>/ k$,

$$
\begin{aligned}
& P_{n}=\frac{1}{<n>+1}\left(\frac{<n>}{<n>+1}\right)^{n} \\
& =\left(e^{\beta \hbar \omega / 2}-e^{-\beta \hbar \omega / 2}\right) e^{-\beta \hbar \omega(n+1 / 2)}, T=\frac{\hbar \omega}{\ln \frac{<n>+1}{<n>}} \\
& \sum_{n \geq 0} P_{n}=1, \sum n P_{n}=<n>=\frac{1}{e^{\beta \hbar \omega-1}}, T \simeq \hbar \omega<n>,<n>\gg 1, \\
& P(x)=\sum_{n} x^{n} P_{n}=(1+<n>(1-x))^{-1} .
\end{aligned}
$$

This is easily seen from the generating function in ([0.3), remembering that the generating function of a sum of independent random variables is the product of their generating functions.

Indeed, for

$$
n=n_{1}+n_{2}+\ldots+n_{k}
$$

with $n_{i}$ independent of each other, the probability distribution of $n$ is

$$
\begin{aligned}
& P_{n}=\sum_{n_{1}, \ldots, n_{k}} \delta\left(n-\sum n_{i}\right) p_{n_{1}} \ldots p_{n_{k}}, \\
& P(x)=\sum_{n} x^{n} P_{n}=p(x)^{k}
\end{aligned}
$$

This has a consequence that an incoherent superposition of $\mathrm{N}$ emitters that have a negative binomial distribution with parameters $k,<n>$ produces a negative binomial distribution with parameters $N k, N<n>$.

So, for the GF of NBD we have $(\mathrm{N}=2)$

$$
F(k,<n>) F(k,<n>)=F(2 k, 2<n>)
$$

And more general formula $(\mathrm{N}=\mathrm{m})$ is

$$
F(k,<n>)^{m}=F(m k, m<n>)
$$

We can put this equation in the closed nonlocal form

$$
Q_{q} F=F^{q},
$$

where

$$
Q_{q}=q^{D}, D=\frac{k d}{d k}+\frac{<n>d}{d<n>}=\frac{x_{1} d}{d x_{1}}+\frac{x_{2} d}{d x_{2}}
$$


Note that temperature defined in (10.22) gives an estimation of the Glukvar temperature when it radiates hadrons. If we take $\hbar \omega=100 \mathrm{MeV}$, to $T \simeq T_{c} \simeq 200 \mathrm{MeV}$ corresponds $<n>\simeq 1.5$ If we take $\hbar \omega=10 \mathrm{MeV}$, to $T \simeq T_{c} \simeq 200 \mathrm{MeV}$ corresponds $\langle n>\simeq 20$. A singular behavior of $\langle n>$ may indicate corresponding phase transition and temperature. At that point we estimate characteristic quantum $\hbar \omega$.

We see that universality of NBD in hadron-production is similar to the universality of black body radiation.

\section{Multiparticle production stochastic dynamics}

Let us imagine space-time development of the the multiparticle process and try to describe it by some (phenomenological) dynamical equation. We start to find the equation for the Poisson distribution and than naturally extend them for the NBD case.

Let us define an integer valued variable $n(t)$ as a number of events (produced particles) at the time $t, n(0)=0$. The probability of event $n(t), P(t, n)$, is defined from the following motion equation

$$
\begin{aligned}
& P_{t} \equiv \frac{\partial P(t, n)}{\partial t}=r(P(t, n-1)-P(t, n)), n \geq 1 \\
& \left.P_{t}(t, 0)\right)=-r P(t, 0) \\
& P(t, n)=0, n<0
\end{aligned}
$$

so

$$
\begin{aligned}
& P(t, 0) \equiv P_{0}(t)=e^{-r t} \\
& P(t, n)=Q(t, n) P_{0}(t) \\
& Q_{t}(t, n)=r Q(t, n-1), Q(t, 0)=1
\end{aligned}
$$

To solve the equation for $Q$, we invent its generating function

$$
F(t, h)=\sum_{n \geq 0} h^{n} Q(t, n)
$$

and solve corresponding equation

$$
F_{t}=r h F, F(t, h)=e^{r t h}=\sum h^{n} \frac{(r t)^{n}}{n !}, Q(t, n)=\frac{(r t)^{n}}{n !},
$$

so

$$
P(t, n)=e^{-r t} \frac{(r t)^{n}}{n !}
$$

is the Poisson distribution.

If we compare this distribution with ([0.2]), we identify $\langle n\rangle=r t$, as if we have a free particle motion with velocity $r$ and the distance is the mean multiplicity. This way we have a connection between $\mathrm{n}$-dimension of the multiplicity and the usual dimension of trajectory. 
As the equation gives right solution, its generalization may give more general distribution, so we will generalize the equation $(\mathbb{\amalg}$.

$$
\begin{aligned}
& P_{t}(t, n)=r\left(e^{-\partial_{n}}-1\right) P(t, n) \\
& =\sum_{k \geq 1} D_{k} \partial^{k} P(t, n), D_{k}=(-1)^{k} \frac{r}{k !},
\end{aligned}
$$

where the $D_{k}, k \geq 1$, are generalized diffusion coefficients.

\subsection{Fractal dimension of the multiparticle production trajectories}

For other values of the coefficients, we will have other distributions. For mean square deviation of the trajectory we have

$$
<(x-\bar{x})^{2}>=<x^{2}>-<x>^{2} \equiv D(x)^{2} \sim t^{2 / d_{f}},
$$

where $d_{f}$ is fractal dimension. For smooth classical trajectory of particles we have $d_{f}=1$; for free stochastic, Brownian, trajectory, all diffusion coefficients are zero but $D_{2}$, we have $d_{f}=2$. In the case of Poisson process we have,

$$
D(n)^{2}=<n^{2}>-<n>^{2} \sim t, d_{f}=2 .
$$

In the case of the NBD and KNO distributions

$$
D(n)^{2} \sim t^{2}, d_{f}=1 .
$$

As we have seen, rasing $k$, KNO reduce to the Poisson, so we have a dimensional (phase) transition from the phase with dimension 1 to the phase with dimension 2. It is interesting, if somehow this phase transition is connected to the other phase transitions in strong interaction processes. For the Poisson distribution GF is solution of the following equation,

$$
\dot{F}=-r(1-h) F,
$$

For the NBD corresponding equation is

$$
\dot{F}=\frac{-r(1-h)}{1+\frac{r t}{k}(1-h)} F=-R(t) F, R(t)=\frac{r(1-h)}{1+\frac{r t}{k}(1-h)} .
$$

If we change the time variable as $t=T^{d_{f}}$, we reduce the dispersion low from general fractal to the NBD like case. Corresponding transformation for the evolution equation is

$$
F_{T}=-d_{f} T^{d_{f}-1} R\left(T^{d_{F}}\right) F,
$$

we ask that this equation coincides with NBD motion equation, and define rate function $R(T)$

$$
d_{f} T^{d_{f}-1} R\left(T^{d_{F}}\right)=\frac{r(1-h)}{1+\frac{r T}{k}(1-h)}
$$

The following equation defines a production processes with fractal dimension $d_{F}$

$$
F_{t}=-R(t) F, R(t)=\frac{r(1-h)}{d_{F} t^{\frac{d_{F}-1}{d_{F}}}\left(1+\frac{r 1^{1 / d_{F}}}{k}(1-h)\right)}
$$




\section{Dynamical Formulation of $\mathrm{z}$ - Scaling}

Motion equations of physics (applied mathematics in general) connect different observable quantities and reduce the number of independently measurable quantities. More fundamental equation contains less number of independent quantities. When (before) we solve the equations, we invent dimensionless invariant variables, than one solution can describe all of the class of phenomena.

In the $\mathrm{z}$ - Scaling $(\mathrm{zS})$ approach to the inclusive multiparticle distributions (MPD) (see, e.g. [Tokarev, Zborovsky, 2007]]), different inclusive distributions depending on the variables $x_{1}, \ldots x_{n}$, are described by universal function $\Psi(z)$ of fractal variable $z$,

$$
z=x_{1}^{-\alpha_{1}} \ldots x_{n}^{-\alpha_{n}} .
$$

It is interesting to find a dynamical system which generates this distributions and describes corresponding MPD.

We can find a good function if we know its derivative. Let us consider the following RD like equation

$$
\begin{aligned}
& z \frac{d}{d z} \Psi=V(\Psi), \\
& \int_{\Psi\left(z_{0}\right)}^{\Psi(z)} \frac{d x}{V(x)}=\ln \frac{z}{z_{0}}
\end{aligned}
$$

\subsection{Formal motivation (foundation) of the RD motion equation for $\Psi$}

As a dimensionless physical quantity $\Psi(z)$ may depend only on the running coupling constant $g(\tau), \tau=\ln z / z_{0}$

$$
\begin{aligned}
& z \frac{d}{d z} \Psi=\dot{\Psi}=\frac{d \Psi}{d g} \beta(g)=U(g)=U\left(f^{-1}(\Psi)\right)=V(\Psi) \\
& \Psi(\tau)=f(g(\tau)), g=f^{-1}(\Psi(\tau))
\end{aligned}
$$

\subsection{Realistic solution for $\Psi$}

According to the paper [Tokarev, Zborovsky, 2007], for high values of $z, \Psi(z) \sim z^{-\beta}$; for small $z, \Psi(z) \sim$ const.

So, for high $z$,

$$
z \frac{d}{d z} \Psi=V(\Psi(z))=-\beta \Psi(z)
$$

for smaller values of $z, \Psi(z)$ rise and we expect nonlinear terms in $V(\Psi)$,

$$
V(\Psi)=-\beta \Psi+\gamma \Psi^{2} .
$$

With this function, we can solve the equation for $\Psi$ and find

$$
\Psi(z)=\frac{1}{\frac{\gamma}{\beta}+c z^{\beta}} .
$$




\subsection{More general solution for $\Psi$}

Let us consider more general potential $V$

$$
z \frac{d}{d z} \Psi=V(\Psi)=-\beta \Psi(z)+\gamma \Psi(z)^{1+n}
$$

Corresponding solution for $\Psi$ is

$$
\Psi(z)=\frac{1}{\left(\frac{\gamma}{\beta}+c z^{n \beta}\right)^{\frac{1}{n}}}
$$

More general solution contains three parameters and may better describe the data of inclusive distributions.

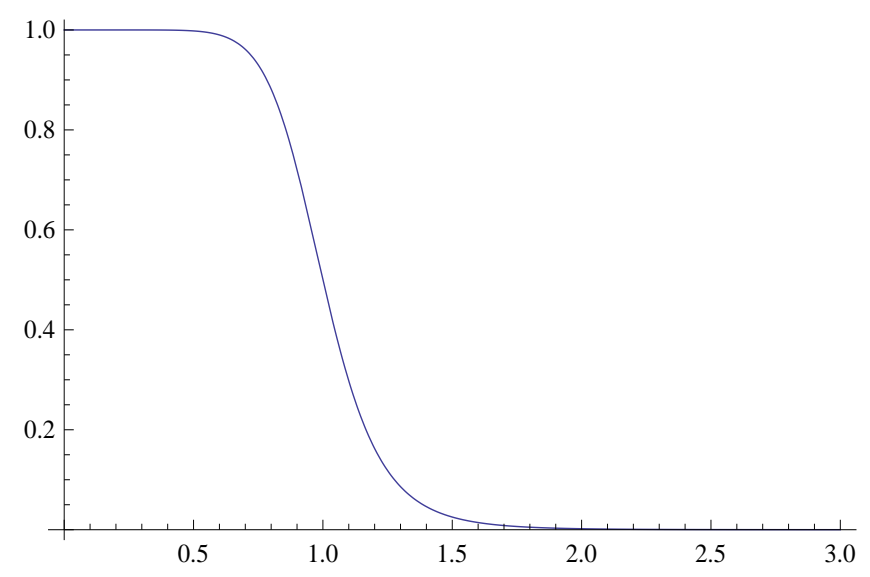

Figure 3: z-scaling distribution $\Psi(z, 9,9,1,1)$

In the case of $n=1$ we reproduce the previous solution.

Another "natural" case is $n=1 / \beta$,

$$
\Psi(z)=\frac{1}{\left(\frac{\gamma}{\beta}+c z\right)^{\beta}}
$$

In this case, we can absorb (interpret) the combined parameter by shift and scaling

$$
z \rightarrow \frac{1}{c}\left(z-\frac{\gamma}{\beta}\right)
$$

Another interesting point of view is to predict the value of $\beta$

$$
\beta=\frac{1}{n}=0.5 ; 0.33 ; 0.25 ; 0.2 ; \ldots, n=2,3,4,5, \ldots
$$

For experimentally suggested value $\beta \simeq 9, n=0.11$

The three parameter function is restricted by the normalization condition

$$
\int_{0}^{\infty} \Psi(z) d z=1
$$




$$
B\left(\frac{\beta-1}{\beta n}, \frac{1}{\beta n}\right)=\left(\frac{\beta}{\gamma}\right)^{\frac{\beta-1}{\beta n}} \frac{\beta n}{c^{\beta n}},
$$

so remains only two free parameter. When $\beta n=1$, we have

$$
c=(\beta-1)\left(\frac{\beta}{\gamma}\right)^{\beta-1}
$$

If $\beta n=1$ and $\beta=\gamma$, than $c=\beta-1$.

In general

$$
c^{\beta n}=\left(\frac{\beta}{\gamma}\right)^{\frac{\beta-1}{\beta n}} \frac{\beta n}{B\left(\frac{\beta-1}{\beta n}, \frac{1}{\beta n}\right)}
$$

\subsection{Space-time dimension inside hadrons and nuclei}

The dimension of the space(-time) is the model dependent concept. E.g. for the fundamental bosonic string model (in flat space-time) the dimension is 26; for superstring model the dimension is 10 [Kaku, 2000].

Let us imagine that we have some action-functional formulation with the fundamental motion equation

$$
z \frac{d}{d z} \Psi=V(\Psi(z))=V(\Psi)=-\beta \Psi+\gamma \Psi^{1+n}
$$

Than, the corresponding Lagrangian contains the following mass and interaction parts

$$
-\frac{\beta}{2} \Psi^{2}+\frac{\gamma}{2+n} \Psi^{2+n}
$$

The action gives renormalizable (effective quantum field theory) model when

$$
d+2=\frac{2 N}{N-2}=\frac{2(2+n)}{n}=2+\frac{4}{n}=2+4 \beta,
$$

so, measuring the parameter $\beta$ inside hadronic and nuclear matters, we find corresponding (fractal) dimension.

\subsection{Another action formulation of the Fundamental equation}

From fundamental equation we obtain

$$
\begin{aligned}
& \left(z \frac{d}{d z}\right)^{2} \Psi \equiv \ddot{\Psi}=V^{\prime}(\Psi) V(\Psi)=\frac{1}{2}\left(V^{2}\right)^{\prime} \\
& =\beta^{2} \Psi-\beta \gamma(n+2) \Psi^{n+1}+\gamma^{2}(n+1) \Psi^{2 n+1}
\end{aligned}
$$

Corresponding action Lagrangian is

$$
\begin{aligned}
& L=\frac{1}{2} \dot{\Psi}^{2}+U(\Psi), U=\frac{1}{2} V^{2}=\frac{1}{2} \Psi^{2}\left(\beta-\gamma \Psi^{n}\right)^{2} \\
& =\frac{\beta^{2}}{2} \Psi^{2}-\beta \gamma \Psi^{2+n}+\frac{\gamma^{2}}{2} \Psi^{2+2 n}
\end{aligned}
$$


This potential, $-U$, has two maximums, when $V=0$, and minimum, when $V^{\prime}=0$, at $\Psi=0$ and $\Psi=(\beta / \gamma)^{1 / n}$, and $\Psi=(\beta /(n+1) \gamma)^{1 / n}$, correspondingly.

We define time-space-scale field $\Psi(t, x, \eta)$, where $\eta=\ln z$ - is scale coordinate variable, with corresponding action functional

$$
A=\int d t d^{d} x d \eta\left(\frac{1}{2} g^{a b} \partial_{a} \Psi \partial_{b} \Psi+U(\Psi)\right)
$$

The renormalization constraint for this action is

$$
N=2+2 n=\frac{2(2+d)}{2+d-2}=2+\frac{4}{d}, d n=2, d=2 / n=2 \beta .
$$

So we have two models for spase-time dimension, (12.17) and (12.21),

$$
d_{1}=4 \beta ; d_{2}=2 \beta
$$

The coordinate $\eta$ characterise (multiparticle production) physical process at the (external) spacetime point $(\mathrm{x}, \mathrm{t})$. The dimension of the space-time inside hadrons and nuclei, where multiparticle production takes place is

$$
d+1=1+2 \beta
$$

Note that this formula reminds the dimension of the spin s state, $d_{s}=2 s+1$. If we take $\beta(=s)=$ $0 ; 1 / 2 ; 1 ; 3 / 2 ; 2 ; \ldots$ We will have $d+1=1 ; 2 ; 3 ; 4 ; 5 ; \ldots$

\section{Zeros of the Riemann zeta function}

The Riemann zeta function $\zeta(s)$ is defined for complex $s=\sigma+i t$ and $\sigma>1$ by the expansion

$$
\begin{aligned}
& \zeta(s)=\sum_{n \geq 1} n^{-s}, \operatorname{Re} s>1, \\
& =\left.\delta_{x}^{-s} \frac{x}{1-x}\right|_{x \rightarrow 1}=\left.\frac{1}{\Gamma(s)} \int_{0}^{\infty} t^{s-1} e^{-\delta_{x} t} \frac{x}{1-x}\right|_{x \rightarrow 1}=\left.\frac{1}{\Gamma(s)} \int_{0}^{\infty} t^{s-1} e^{t \partial_{\tau}} \frac{1}{e^{\tau}-1}\right|_{\tau \rightarrow 0} \\
& =\frac{1}{\Gamma(s)} \int_{0}^{\infty} \frac{t^{s-1} d t}{e^{t}-1}, x=e^{-\tau} .
\end{aligned}
$$

All complex zeros, $s=\alpha+i \beta$, of $\zeta(\sigma+i t)$ function lie in the critical stripe $0<\sigma<1$, symmetrically with respect to the real axe and critical line $\sigma=1 / 2$. So it is enough to investigate zeros with $\alpha \leq 1 / 2$ and $\beta>0$. These zeros are of three type, with small, intermediate and big ordinates.

\subsection{Riemann hypothesis}

The Riemann hypothesis [Titchmarsh, 1986] states that the (non-trivial) complex zeros of $\zeta(s)$ lie on the critical line $\sigma=1 / 2$.

At the beginning of the XX century Polya and Hilbert made a conjecture that the imaginary part of the Riemann zeros could be the oscillation frequencies of a physical system ( $\zeta$ - (mem)brane).

After the advent of Quantum Mechanics, the Polya-Hilbert conjecture was formulated as the existence of a self-adjoint operator whose spectrum contains the imaginary part of the Riemann zeros.

The Riemann hypothesis (RH) is a central problem in Pure Mathematics due to its connection with Number theory and other branches of Mathematics and Physics. 


\subsection{The functional equation for zeta function}

The functional equation is (see e.g. [Titchmarsh, 1986])

$$
\zeta(1-s)=\frac{2 \Gamma(s)}{(2 \pi)^{s}} \cos \left(\frac{\pi s}{2}\right) \zeta(s)
$$

From this equation we see the real (trivial) zeros of zeta function:

$$
\zeta(-2 n)=0, n=1,2, \ldots
$$

Also, at $\mathrm{s}=1$, zeta has pole with reside 1 .

From Field theory and statistical physics point of view, the functional equation $(\mathbb{L} 3.2)$ is duality relation, with self dual (or critical) line in the complex plane, at $s=1 / 2+i \beta$,

$$
\zeta\left(\frac{1}{2}-i \beta\right)=\frac{2 \Gamma(s)}{(2 \pi)^{s}} \cos \left(\frac{\pi s}{2}\right) \zeta\left(\frac{1}{2}+i \beta\right),
$$

we see that complex zeros lie symmetrically with respect to the real axe.

On the critical line, (nontrivial) zeros of zeta corresponds to the infinite value of the free energy,

$$
F=-T \ln \zeta
$$

At the point with $\beta=14.134725 \ldots$ is located the first zero. In the interval $10<\beta<100$, zeta has 29 zeros. The first few million zeros have been computed and all lie on the critical line. It has been proved that uncountably many zeros lie on critical line.

The first relation of zeta function with prime numbers is given by the following formula,

$$
\zeta(s)=\prod_{p}\left(1-p^{-s}\right)^{-1}, \operatorname{Re} s>1 .
$$

Another formula, which can be used on critical line, is

$$
\begin{aligned}
& \zeta(s)=\left(1-2^{1-s}\right)^{-1} \sum_{n \geq 1}(-1)^{n+1} n^{-s}, \text { Re } s>0 \\
& =\left.\frac{e^{i \pi\left(\delta_{x}+1\right)}}{\left(1-2^{1-s}\right) \delta_{x}^{s}} \frac{x}{1-x}\right|_{x \rightarrow 1}=\left.\frac{1}{1-2^{1-s}} \frac{1}{\Gamma(s)} \int_{0}^{\infty} d t t^{s-1} e^{i \pi} e^{(i \pi-t) \delta_{x}} \frac{1}{x^{-1}-1}\right|_{x \rightarrow 1} \\
& =\left.\frac{1}{1-2^{1-s}} \frac{1}{\Gamma(s)} \int_{0}^{\infty} d t t^{s-1} e^{(t-i \pi) \partial_{\tau}} \frac{e^{i \pi}}{e^{\tau}-1}\right|_{\tau \rightarrow 0}=\frac{1}{1-2^{1-s}} \frac{1}{\Gamma(s)} \int_{0}^{\infty} \frac{t^{s-1} d t}{e^{t}+1}, \\
& \int_{0}^{\infty} \frac{t^{s-1} d t}{e^{t}+1}=\int_{0}^{\infty} d t t^{s-1} e^{-t} \sum_{n \geq 0}(-1)^{n} e^{-n t}=\Gamma(s) \sum_{n \geq 1}(-1)^{n+1} n^{-s}
\end{aligned}
$$

\subsection{From Qlike to zeta equations}

Let us consider the values $q=n, n=1,2,3, \ldots$ and take sum of the corresponding equations (25.139), we find

$$
\zeta(-D) F=\frac{F}{1-F}
$$


In the case of the NBD we know the solutions of this equation.

Now we invent a Hamiltonian $H$ with spectrum corresponding to the set of nontrivial zeros of the zeta function, in correspondence with Riemann hypothesis,

$$
\begin{aligned}
& -D_{n}=\frac{n}{2}+i H_{n}, H_{n}=i\left(\frac{n}{2}+D_{n}\right), \\
& D_{n}=x_{1} \partial_{1}+x_{2} \partial_{2}+\ldots+x_{n} \partial_{n}, H_{n}^{+}=H_{n}=\sum_{m=1}^{n} H_{1}\left(x_{m}\right), \\
& H_{1}=i\left(\frac{1}{2}+x \partial_{x}\right)=-\frac{1}{2}(x \hat{p}+\hat{p} x), \hat{p}=-i \partial_{x}
\end{aligned}
$$

The Hamiltonian $H=H_{n}$ is hermitian, its spectrum is real. The case $n=1$ corresponds to the Riemann hypothesis.

The case $n=2$, corresponds to NBD,

$$
\begin{aligned}
& \zeta\left(1+i H_{2}\right) F=\frac{F}{1-F},\left.\zeta\left(1+i H_{2}\right)\right|_{F}=\frac{1}{1-F}, \\
& F\left(x_{1}, x_{2} ; h\right)=\left(1+\frac{x_{1}}{x_{2}}(1-h)\right)^{-x_{2}}
\end{aligned}
$$

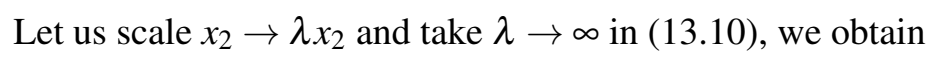

$$
\begin{aligned}
& \zeta\left(\frac{1}{2}+i H_{1}(x)\right) e^{-(1-h) x}=\frac{1}{e^{(1-h) x}-1}, \\
& \frac{1}{\zeta\left(\frac{1}{2}+i H(x)\right)} \frac{1}{e^{\varepsilon x}-1}=e^{-\varepsilon x}, \\
& H(x)=i\left(\frac{1}{2}+x \partial_{x}\right)=-\frac{1}{2}(x \hat{p}+\hat{p} x), H^{+}=H, \varepsilon=1-h .
\end{aligned}
$$

Now we scale $x \rightarrow x y$, multiply the equation by $y^{s-1}$ and integrate

$$
\begin{aligned}
& \frac{1}{\zeta\left(\frac{1}{2}+i H(x)\right)} \int_{0}^{\infty} d y \frac{y^{s-1}}{e^{\varepsilon x y}-1}=\int_{0}^{\infty} d y e^{-\varepsilon x y} y^{s-1}=\frac{1}{(\varepsilon x)^{s}} \Gamma(s), \\
& \frac{1}{\zeta\left(\frac{1}{2}+i H(x)\right)} \int_{0}^{\infty} d y \frac{y^{s-1}}{e^{\varepsilon x y}-1}=\frac{1}{\zeta\left(\frac{1}{2}+i H(x)\right)} x^{-s} \varepsilon^{-s} \Gamma(s) \zeta(s),
\end{aligned}
$$

so

$$
\begin{aligned}
& \zeta\left(\frac{1}{2}+i H(x)\right) x^{-s}=\zeta(s) x^{-s} \Rightarrow H(x) \psi_{E}=E \psi_{E}, \\
& \psi_{E}=c x^{-s}, s=\frac{1}{2}+i E
\end{aligned}
$$

we have correct way and can return to the previous step $(\mathbb{L} 3 . \mathbb{}$ ) and take the following transformation

$$
\begin{aligned}
& \frac{1}{e^{\varepsilon x y}-1}=\frac{1}{2 \pi} \int_{-\infty+i c}^{\infty+i c} d E x^{-i E-1 / 2} \varphi(E, \varepsilon y), \\
& \varphi(E, \varepsilon y)=\int_{0}^{\infty} d x \frac{x^{i E-\frac{1}{2}}}{e^{\varepsilon x y}-1}=\frac{\Gamma\left(\frac{1}{2}+i E\right)}{(\varepsilon y)^{i E+1 / 2}} \zeta\left(\frac{1}{2}+i E\right), \\
& \frac{1}{2 \pi} \int_{-\infty+i c}^{\infty+i c} d E x^{-i E-1 / 2} \varphi(E, \varepsilon y) \frac{1}{\zeta(1 / 2+i E)}=e^{-\varepsilon x y}
\end{aligned}
$$


If we take the following formula

$$
\zeta(s)=\frac{1}{\Gamma(s)} \int_{0}^{\infty} \frac{t^{s-1} d t}{e^{t}-1},
$$

which says that $\zeta$ function is the Mellin transformation, we can find

$$
\Gamma\left(1+i H_{2}\right) \frac{F}{1-F}=\int_{0}^{\infty} \frac{d t / t}{e^{t}-1} F^{1 / t}
$$

or

$$
\begin{aligned}
& \Gamma\left(1+i H_{2}\right) \Phi=\int_{0}^{\infty} \frac{d t / t}{e^{t}-1}\left(\frac{\Phi}{1+\Phi}\right)^{1 / t} \\
& \Phi=\frac{F}{1-F}=\frac{1}{\left(1+\frac{x_{1}}{x_{2}}(1-h)\right)^{x_{2}}-1}
\end{aligned}
$$

We can obtain also the following equation with argument of $\zeta_{N}$ on critical axis

$$
\begin{aligned}
& \zeta_{N}\left(\frac{1}{2}+i H_{1}\left(x_{2}\right)\right) F\left(x_{1}, x_{2}, h\right)=\sum_{n=1}^{N} \frac{1}{\left(1+\frac{x_{1}}{n x_{2}}(1-h)\right)^{n x_{2}}} \\
& =\sum_{n=1}^{N} F\left(x_{1}, n x_{2}, h\right) \\
& \zeta_{N}\left(\frac{1}{2}+i H_{1}\left(x_{2}\right)\right) F\left(\lambda x_{1}, x_{2}, h\right)=\sum_{n=1}^{N} \frac{1}{\left(1+\frac{\lambda x_{1}}{n x_{2}}(1-h)\right)^{n x_{2}}} \\
& =\sum_{n=1}^{N} F\left(\lambda x_{1}, n x_{2}, h\right) \simeq N e^{-\lambda(1-h) x_{1}}, N \gg 1 .
\end{aligned}
$$

Let us calculate next therm in the $1 / \lambda$ expansion in the ([13.10)

$$
\begin{aligned}
& F\left(x_{1}, \lambda x_{2}, h\right)=\left(1+\frac{\varepsilon x_{1}}{\lambda x_{2}}\right)^{-\lambda x_{2}}=e^{-\lambda x_{2} \ln \left(1+\varepsilon \frac{x_{1}}{\lambda x_{2}}\right)} \\
& =e^{-\varepsilon x_{1}} e^{\frac{\left(\varepsilon x_{1}\right)^{2}}{2 \lambda x_{2}}+O\left(\lambda^{-2}\right)}=e^{-\varepsilon x_{1}}\left(1+\frac{\left(\varepsilon x_{1}\right)^{2}}{2 \lambda x_{2}}+O\left(\lambda^{-2}\right)\right), \\
& \left(F^{-1}-1\right)^{-1}=\left(e^{\lambda x_{2} \ln \left(1+\varepsilon \frac{x_{1}}{\lambda x_{2}}\right)}-1\right)^{-1} \\
& =\frac{1}{e^{\varepsilon x_{1}}-1}\left(1+\frac{e^{\varepsilon x_{1}}}{e^{\varepsilon x_{1}}-1} \frac{\left(\varepsilon x_{1}\right)^{2}}{2 \lambda x_{2}}+O\left(\lambda^{-2}\right)\right)
\end{aligned}
$$

The zero order term, $\lambda^{0}$ we already considered. The next, $\lambda^{-1}$ order term gives the following relations

$$
\begin{aligned}
& \zeta\left(-\delta_{1}-\delta_{2}\right) \frac{x_{1}^{2}}{x_{2}} e^{-\varepsilon x_{1}}=\frac{1}{x_{2}} \zeta\left(1-\delta_{1}\right) x_{1}^{2} e^{-\varepsilon x_{1}}=\frac{x_{1}^{2} e^{\varepsilon x_{1}}}{x_{2}\left(e^{\varepsilon x_{1}}-1\right)^{2}}, \\
& \zeta(1-\delta) x^{2} e^{-\varepsilon x}=\frac{x^{2} e^{\varepsilon x}}{\left(e^{\varepsilon x}-1\right)^{2}}=x^{2} e^{-\varepsilon x}+O\left(e^{-2 \varepsilon x}\right) \\
& \zeta(1-\delta) \Psi=E \Psi+O\left(e^{-2 \varepsilon x}\right), \Psi=x^{2} e^{-\varepsilon x}, E=1 .
\end{aligned}
$$

There have been a number of approaches to understanding the Riemann hypothesis based on physics (for a comprehensive list see [Watkins]) 
According to the idea of Berry and Keating, [Berry, Keating, 1997] the real solutions $E_{n}$ of

$$
\zeta\left(\frac{1}{2}+i E_{n}\right)=0,
$$

are energy levels, eigenvalues of a quantum Hermitian operator (the Riemann operator) associated with the one-dimensional classical hyperbolic Hamiltonian

$$
H_{c}=x p
$$

where $x$ and $p$ are the conjugate coordinate and momentum.

They suggest a quantization condition generating Riemann zeros. This Hamiltonian breaks time-reversal invariance since $(x, p) \rightarrow(x,-p) \Rightarrow H \rightarrow-H$. The classical Hamiltonian $H=x p$ of linear dilation, i.e. multiplication in $x$ and contraction in $p$, gives the Hamiltonian equations:

$$
\begin{aligned}
& \dot{x}=x, \\
& \dot{p}=-p,
\end{aligned}
$$

with the solution

$$
\begin{aligned}
& x(t)=x_{0} e^{t}, \\
& p(t)=p_{0} e^{-t}
\end{aligned}
$$

for any nonzero $E=x_{0} p_{0}=x(t) p(t)$ is hyperbola in phase space.

The system is quantized by considering the dilation operator in the $\mathrm{x}$ space

$$
H=\frac{1}{2}(x p+p x)=-i \hbar\left(\frac{1}{2}+x \partial_{x}\right),
$$

which is the simplest formally Hermitian operator corresponding to the classical Hamiltonian. The eigenvalue equation

$$
H \psi_{E}=E \psi_{E},
$$

is satisfied by the eigenfunctions

$$
\psi_{E}(x)=c x^{-\frac{1}{2}+\frac{i}{\hbar} E},
$$

where the complex constant $c$ is arbitrary, since the solutions are not square-integrable. To the normalization

$$
\int_{0}^{\infty} d x \psi_{E}(x)^{*} \psi_{E^{\prime}}(x)=\delta\left(E-E^{\prime}\right)
$$

corresponds $c=1 / \sqrt{2 \pi}$.

We have seen that

$$
\begin{aligned}
& \zeta\left(\frac{1}{2}+i H\right) e^{-\varepsilon x}=\frac{1}{e^{\varepsilon x}-1}, \\
& H=-i\left(\frac{1}{2}+x \partial_{x}\right)=x^{1 / 2} p x^{1 / 2}, p=-i \partial_{x},
\end{aligned}
$$


than

$$
\begin{aligned}
& e^{-\varepsilon x}=\int d E x^{-1 / 2+i E} \varphi(E, \varepsilon), \\
& \varphi(E, \varepsilon)=\frac{1}{2 \pi} \int_{0}^{\infty} d x x^{-1 / 2-i E} e^{-\varepsilon x}=\frac{\varepsilon^{-1 / 2+i E}}{2 \pi} \Gamma(1 / 2-i E) ; \\
& \zeta\left(\frac{1}{2}+i E\right) \varphi(E, \varepsilon)=\frac{1}{2 \pi} \int_{0}^{\infty} d x \frac{x^{-1 / 2-i E}}{e^{\varepsilon x}-1} \\
& =\frac{\varepsilon^{-1 / 2+i E}}{2 \pi} \Gamma(1 / 2-i E) \zeta\left(\frac{1}{2}-i E\right) .
\end{aligned}
$$

\subsection{Some calculations with zeta function values}

From the equation ([1].]1) we have

$$
\begin{aligned}
& \zeta\left(\frac{1}{2}+i H_{1}(x)\right) e^{-\varepsilon x}=\frac{1}{e^{\varepsilon x}-1}, H_{1}=i\left(\frac{1}{2}+x \partial_{x}\right) \\
& \zeta\left(-x \partial_{x}\right)\left(1-\varepsilon x+\frac{(\varepsilon x)^{2}}{2}+\ldots\right)=\frac{1}{\varepsilon x}\left(1-\left(\frac{\varepsilon x}{2}+\frac{(\varepsilon x)^{2}}{6}+\ldots\right)+\right. \\
& \left.+\left(\frac{\varepsilon x}{2}+\ldots\right)^{2}+\ldots\right)
\end{aligned}
$$

so

$$
\zeta(0)=-\frac{1}{2}, \zeta(-1)=-\frac{1}{12}, \ldots
$$

Note that, a little calculation shows that, the $(\varepsilon x)^{2}$ terms cancels on the r.h.s, in accordance with $\zeta(-2)=0$.

More curious question concerns with the term $1 / \varepsilon x$ on the r.h.s. To it corresponds the term with actual infinitesimal coefficient on the 1.h.s.

$$
\frac{1}{\zeta(1)} \frac{1}{\varepsilon x}
$$

in the spirit of the nonstandard analysis (see, e.g. [Davis, 1977]), we can imagine that such a terms always present but on the r.h.s we may not note them.

For other values of zeta function we will use the following expansion

$$
\begin{aligned}
& \frac{1}{e^{x}-1}=\frac{1}{x+\frac{x^{2}}{2}+\frac{x^{3}}{3 !}+\ldots}=\frac{1}{x}-\frac{1}{2}+\sum_{k \geq 1} \frac{B_{2 k} x^{2 k-1}}{(2 k) !} \\
& B_{2}=\frac{1}{6}, B_{4}=-\frac{1}{30}, B_{6}=\frac{1}{42}, \ldots
\end{aligned}
$$

and obtain

$$
\zeta(1-2 n)=-\frac{B_{2 n}}{2 n}, n \geq 1
$$

\section{Hamiltonization of the General Dynamical Systems}

Let us consider a general dynamical system described by the following system of the ordinary differential equations [Arnold, 1978]

$$
\dot{x}_{n}=v_{n}(x), 1 \leq n \leq N,
$$


$\dot{x}_{n}$ stands for the total derivative with respect to the parameter $\mathrm{t}$.

When the number of the degrees of freedom is even, and

$$
v_{n}(x)=\varepsilon_{n m} \frac{\partial H_{0}}{\partial x_{m}}, 1 \leq n, m \leq 2 M,
$$

the system (ㅍ․ㄱ) is Hamiltonian one and can be put in the form

$$
\dot{x}_{n}=\left\{x_{n}, H_{0}\right\}_{0},
$$

where the Poisson bracket is defined as

$$
\{A, B\}_{0}=\varepsilon_{n m} \frac{\partial A}{\partial x_{n}} \frac{\partial B}{\partial x_{m}}=A \frac{\overleftarrow{\partial}}{\partial x_{n}} \varepsilon_{n m} \frac{\vec{\partial}}{\partial x_{m}} B
$$

and summation rule under repeated indices has been used.

Let us consider the following Lagrangian

$$
L=\left(\dot{x}_{n}-v_{n}(x)\right) \psi_{n}
$$

and the corresponding equations of motion

$$
\dot{x}_{n}=v_{n}(x), \dot{\psi}_{n}=-\frac{\partial v_{m}}{\partial x_{n}} \psi_{m} .
$$

The system (114.6) extends the general system (114.01) by linear equation for the variables $\psi$. The extended system can be put in the Hamiltonian form [Makhaldiani, Voskresenskaya, 1997]

$$
\dot{x}_{n}=\left\{x_{n}, H_{1}\right\}_{1}, \dot{\psi}_{n}=\left\{\psi_{n}, H_{1}\right\}_{1},
$$

where first level (order) Hamiltonian is

$$
H_{1}=v_{n}(x) \psi_{n}
$$

and (first level) bracket is defined as

$$
\{A, B\}_{1}=A\left(\frac{\overleftarrow{\partial}}{\partial x_{n}} \frac{\vec{\partial}}{\partial \psi_{n}}-\frac{\overleftarrow{\partial}}{\partial \psi_{n}} \frac{\vec{\partial}}{\partial x_{n}}\right) B
$$

Note that when the Grassmann grading [Berezin, 1987] of the conjugated variables $x_{n}$ and $\psi_{n}$ are different, the bracket (14.9) is known as Buttin bracket[Buttin, 1996].

In the Faddeev-Jackiw formalism [Faddeev, Jackiw, 1988] for the Hamiltonian treatment of systems defined by first-order Lagrangians, i.e. by a Lagrangian of the form

$$
L=f_{n}(x) \dot{x}_{n}-H(x),
$$

motion equations

$$
f_{m n} \dot{x}_{n}=\frac{\partial H}{\partial x_{m}},
$$


for the regular structure function $f_{m n}$, can be put in the explicit hamiltonian (Poisson; Dirac) form

$$
\dot{x}_{n}=f_{n m}^{-1} \frac{\partial H}{\partial x_{m}}=\left\{x_{n}, x_{m}\right\} \frac{\partial H}{\partial x_{m}}=\left\{x_{n}, H\right\}
$$

where the fundamental Poisson (Dirac) bracket is

$$
\left\{x_{n}, x_{m}\right\}=f_{n m}^{-1}, f_{m n}=\partial_{m} f_{n}-\partial_{n} f_{m} .
$$

The system ([14.6) is an important example of the first order regular hamiltonian systems. Indeed, in the new variables,

$$
y_{n}^{1}=x_{n}, y_{n}^{2}=\psi_{n}
$$

lagrangian ([4.5) takes the following first order form

$$
\begin{aligned}
& L=\left(\dot{x}_{n}-v_{n}(x)\right) \psi_{n} \Rightarrow \frac{1}{2}\left(\dot{x}_{n} \psi_{n}-\dot{\psi}_{n} x_{n}\right)-v_{n}(x) \psi_{n} \\
& =\frac{1}{2} y_{n}^{a} \varepsilon^{a b} \dot{y}_{n}^{b}-H(y) \\
& =f_{n}^{a}(y) \dot{y}_{n}^{a}-H(y), f_{n}^{a}=\frac{1}{2} y_{n}^{b} \varepsilon^{b a}, H=v_{n}\left(y^{1}\right) y_{n}^{2} \\
& f_{n m}^{a b}=\frac{\partial f_{m}^{b}}{\partial y_{n}^{a}}-\frac{\partial f_{n}^{a}}{\partial y_{m}^{b}}=\varepsilon^{a b} \delta_{n m}
\end{aligned}
$$

corresponding motion equations and the fundamental Poisson bracket are

$$
\dot{y}_{n}^{a}=\varepsilon_{a b} \delta_{n m} \frac{\partial H}{\partial y_{m}^{b}}=\left\{y_{n}^{a}, H\right\},\left\{y_{n}^{a}, y_{m}^{b}\right\}=\varepsilon_{a b} \delta_{n m} .
$$

To the canonical quantization of this system corresponds

$$
\left[\hat{y}_{n}^{a}, \hat{y}_{m}^{b}\right]=i \hbar \varepsilon_{a b} \delta_{n m}, \hat{y}_{n}^{1}=y_{n}^{1}, \hat{y}_{n}^{2}=-i \hbar \frac{\partial}{\partial y_{n}^{1}}
$$

In this quantum theory, classical part, motion equations for $y_{n}^{1}$, remain classical.

\section{Hamiltonization of the fractal dynamical systems}

Let us consider the following fractal generalization of the usual finite dimensional dynamical systems

$$
{ }_{0} D_{t}^{-\alpha} x_{n}=f_{n}(x), 1 \leq n \leq N
$$

The Hamiltonian extension of the dynamical system we construct from the following action functional

$$
\begin{aligned}
& S=\int_{0}^{T} d t \psi_{n}(t)\left({ }_{0} D_{t}^{-\alpha} x_{n}-f_{n}(x)\right)=\int_{0}^{T} d t \psi_{n}(t)\left({ }_{0} D_{t}^{-\alpha} x_{n}\right)-H \\
& =\int_{0}^{T} d t x_{n}(t)\left({ }_{t} D_{T}^{-\alpha} \psi_{n}\right)-H=\left(\int_{0}^{T} d t x_{n}(t)\right)\left({ }_{0} D_{T}^{-\alpha} \psi_{n}\right)-\int_{0}^{T} d t x_{n}(t)\left({ }_{0} D_{t}^{-\alpha} \psi_{n}\right)-H,
\end{aligned}
$$




$$
{ }_{t} D_{T}^{-\alpha}={ }_{0} D_{T}^{-\alpha}-{ }_{0} D_{t}^{-\alpha},{ }_{0} D_{t}^{-\alpha} f=\frac{1}{\Gamma(\alpha)} \int_{0}^{t}|t-\tau|^{\alpha-1} f(\tau)
$$

and complementary linear equation

$$
{ }_{t} D_{T}^{-\alpha} \psi_{n}=\frac{\partial f_{m}}{\partial x^{n}} \psi_{m}
$$

and, when

$$
{ }_{0} D_{T}^{-\alpha} \psi_{n}=0 \Rightarrow \frac{\partial f_{m}}{\partial x^{n}} \psi_{m}(0)
$$

we obtain

$$
{ }_{0} D_{t}^{-\alpha} \psi_{n}=-\frac{\partial f_{m}}{\partial x^{n}} \psi_{m}
$$

Note that for classical Hamiltonization we have the following boundary conditions

$$
\psi(0) x(0)=\psi(T) x(T)
$$

\subsection{Supersymmetric Classical and Quantum Dynamics}

Supersymmetric classical and quantum mechanics (SQM) appear after supersymmetric field theories, [Berezin, 1987], [Witten, 1998]]. At its veryroots the conceptual foundations of quantum theory involve notions of discreteness and uncertainty. Schroedinger and Heisenberg, respectively, gave two distinct but equivalent formulations: the configuration space approach which deals with wave functions and the phase space approach which focuses on the role of observables. Dirac noticed a connection between commutators and classical Poisson brackets and it was chiefly who gave the commutator form of the Poisson bracket in quantum mechanics on the basis of Bohr's correspondence principle. In field theory supersymmetry was invented bay Gol'fand and Likhtam [Gol'fand, Likhtman, 197], in string theory supersymmetry was invented by Ramond and Neveu and Schwarz [Ramond, 1971], [Neveu,Schwarz,1971] ]. Subsequent to these works various models embedding SUSY were proposed within a field-theoretic framework. The most notable one was the work of Wess and Zumino [Wess,Zumino, 1974] who defined a set of supergauge transformation in four space-time dimensions and pointed out their relevance to the Lagrangian free-field theory. It has been found that SUSY field theories prove to be the least divergent in comparison with the usual quantum field theories. From a particle physics point of view, some of the major motivations for the study of SUSY are: (i) it provides a convenient platform for unifying matter and force, (ii) it reduces the divergence of quantum gravity, and (iii) it gives an answer to the so-called "hierarchy problem" in grand unified theories.

\subsection{Reduction of the Higher Order Dynamical System}

Note that the procedure of reduction of the higher order dynamical system, e.g. second order Euler-Lagrange motion equations, to the first order dynamical systems, in the case to the Hamiltonian motion equations, can be continued using fractal calculus. E.g. first order system can be reduced to the half order one,

$$
\begin{aligned}
& D^{1 / 2} q=\psi, \\
& D^{1 / 2} \psi=p \Leftrightarrow \dot{q}=p .
\end{aligned}
$$




\subsection{Dynamics and Superdynamics}

Hamiltonian mechanics

$$
\begin{aligned}
& \dot{x}=p \\
& \dot{p}=-V_{x}=x f
\end{aligned}
$$

when $f=k=$ constant, we have the harmonic oscillator case. The Hamiltonian dynamics is the first order reduction of the second order Lagrangian dynamics. The superdynamics is a following $1 / 2$ reduction of the Hamiltonian dynamics in the superspace $(t, \theta)$

$$
\begin{aligned}
& \partial_{t}^{1 / 2}=\partial_{\theta}+\theta \partial_{t},\left(\partial_{t}^{1 / 2}\right)^{2}=\partial_{t}, x(t, \theta)=x_{0}(t)+\theta x_{1}(t) \\
& \partial_{t}^{1 / 2} x=y, \\
& \partial_{t}^{1 / 2} y=u=p, \\
& \partial_{t}^{1 / 2} u=v, \\
& \partial_{t}^{1 / 2} v=f,
\end{aligned}
$$

or, in the matrix form

$$
\partial_{t}^{1 / 2} \psi=A \psi, \psi^{T}=(x, y, u, v), A=\left(\begin{array}{llll}
0 & 1 & 0 & 0 \\
0 & 0 & 1 & 0 \\
0 & 0 & 0 & 1 \\
f & 0 & 0 & 0
\end{array}\right), \operatorname{tr}(A)=0, \operatorname{det}(A)=-f .
$$

In the component form the motion equations and step by step there solutions are

$$
\begin{aligned}
& \text { 8. } \dot{x}_{0}=y_{1}=u_{0}=c_{3}+\int d t f \\
& \text { 3. } \dot{y}_{0}=u_{1}=c_{1} \Rightarrow y_{0}=c_{1} t+c_{2} \\
& \text { 6. } \dot{u}_{0}=v_{1}=f \Rightarrow u_{0}(t)=c_{3}+\int d t f, \\
& \text { 1. } \dot{v}_{0}=0 \Rightarrow v_{0}=c_{1}=\text { const } \\
& \text { 4. } x_{1}=y_{0}=c_{1} t+c_{2}, \\
& \text { 7. } y_{1}=u_{0}, \\
& \text { 2. } u_{1}=v_{0}=c_{1}, \\
& \text { 5. } v_{1}=f .
\end{aligned}
$$

\subsection{Renormdynamics and Superdynamics}

In the dynamical systems of the mechanics the simplest and most fundamental case corresponds to the the system with one dynamical degrees of freedom and two dimensional phase space $(x, p), N=2$. In renormdynamics the symplest and most important case correspondes to the one dimensional phase space, $N=1$. Corresponding examples are given by one charge renormdynamics of QED and QCD,

$$
\dot{x}=f(x)
$$

with the solution in the following implicit form

$$
\int_{x_{0}}^{x(t)} \frac{d x}{f(x)}=t-t_{0}
$$




\subsection{Super-Fractal Dynamics}

Let us consider fractal superdynamics on the superspace $(t, \theta)$

$$
\begin{aligned}
& D x=f(x), D=\partial_{\theta}+\theta \partial_{t}, D^{2}=\partial_{t}, x(t, \theta)=x_{0}(t)+\theta x_{1}(t), \\
& x_{1}+\theta \dot{x}_{0}=f_{0}\left(x_{0}\right)+\theta\left(f_{0}^{\prime}\left(x_{0}\right) x_{1}+f_{1}\left(x_{0}\right)\right), \\
& x_{1}=f_{0}\left(x_{0}\right), \\
& \dot{x}_{0}=f_{0}^{\prime}\left(x_{0}\right) x_{1}+f_{1}\left(x_{0}\right)=f_{0}^{\prime}\left(x_{0}\right) f_{0}\left(x_{0}\right)+f_{1}\left(x_{0}\right)=H_{0}^{\prime}+f_{1}=\varphi\left(x_{0}\right), H_{0}=\frac{1}{2} f_{0}^{2} \\
& \int_{x_{0}}^{x(t)} \frac{d x}{\varphi(x)}=t-t_{0}
\end{aligned}
$$

Note that, if we take, $f_{1}=0$, we will have gradient flow with potential function $H_{0}$

\subsection{Hamiltonian extension of the SFD}

We start from the following action

$$
\begin{aligned}
& S=\int d t d \theta p(t, \theta)(D x-f(x))=\int d t\left(p_{0}(t)\left(\dot{x}_{0}-f_{0}^{\prime}\left(x_{0}\right) x_{1}-f_{1}\left(x_{0}\right)\right)+p_{1}(t)\left(x_{1}-f_{0}\left(x_{0}\right)\right)\right), \\
& p=p_{0}+\theta p_{1}, x=x_{0}+\theta x_{1}, f(x)=f_{0}(x)+\theta f_{1}(x)=f_{0}\left(x_{0}\right)+\theta f_{0}^{\prime}\left(x_{0}\right) x_{1}+\theta f_{1}\left(x_{0}\right)
\end{aligned}
$$

Variations with respect to the $p_{1}$ and $p_{0}$ give the previous equations of motion. Variations with respect to the $x_{1}$ and $x_{0}$ give the following complementary motion equations

$$
\begin{aligned}
& p_{1}=f_{0}^{\prime}\left(x_{0}\right) p_{0}, \\
& \dot{p}_{0}=-f_{0}^{\prime \prime}\left(x_{0}\right) x_{1} p_{0}-f_{1}^{\prime}\left(x_{0}\right)-p_{1} f_{0}^{\prime}\left(x_{0}\right) \\
& =-f_{0}^{\prime \prime} f_{0} p_{0}-f_{1}^{\prime}\left(x_{0}\right)-f_{0}^{\prime 2} p_{0}=-H_{0}^{\prime \prime} p_{0}-f_{1}^{\prime}\left(x_{0}\right)
\end{aligned}
$$

In the case of the gradient flow: $f_{1}=0 \Rightarrow f_{1}^{\prime}=0$ and for $f_{0}=a x+b$ we have the following solution

$$
\begin{aligned}
& p_{0}(t)=A e^{-a^{2} t}, p_{1}=a p_{0}, \\
& x_{0}=B e^{a^{2} t}-b / a, x_{0} \geq b / a, \\
& x_{0}=-B e^{a^{2} t}-b / a, x_{0} \leq b / a, \\
& x_{1}=a x_{0}+b
\end{aligned}
$$

For general solution we have

$$
\begin{aligned}
& p_{0}(t)=A \exp \left(-\int_{0}^{t} d \tau H_{0}^{\prime \prime}\left(x_{0}(\tau)\right)\right)=A \exp \left(-\int_{x_{0}(0)}^{x_{0}(t)} d x H_{0}^{\prime \prime} / H_{0}^{\prime}\right) \\
& =A H_{0}^{\prime}\left(x_{0}(0)\right) / H_{0}^{\prime}\left(x_{0}(t)\right)
\end{aligned}
$$

\subsection{Hamiltonization of the Newton's Method}

Newton's classical method of solution of the equation $f(x)=0$ is based on the iterative process

$$
x_{n+1}=x_{n}-\varphi\left(x_{n}\right), \varphi\left(x_{n}\right)=\frac{f\left(x_{n}\right)}{f^{\prime}\left(x_{n}\right)}
$$

when it converges, $x_{n} \rightarrow x_{c}, f\left(x_{c}\right)=0$. The Hamiltonian extension of this dynamical system is given by the following Lagrangian

$$
L=\sum_{n} \psi_{n}\left(x_{n+1}-x_{n}+\varphi\left(x_{n}\right)\right)
$$


and the motion equation for $\psi_{n}$

$$
\begin{aligned}
& \psi_{n-1}=\psi_{n}\left(1+\varphi^{\prime}\left(x_{n}\right)\right), \\
& \psi_{n+1}=\left(1+\varphi^{\prime}\left(x_{n+1}\right)\right)^{-1} \psi_{n}
\end{aligned}
$$

when $x_{n} \rightarrow x_{c}, \psi_{n} \rightarrow \psi_{c}, \varphi^{\prime}\left(x_{n}\right) \rightarrow 0$.

\subsection{Stability of the states of dynamical systems}

If we have a solution $x_{n}=x_{0 n}$ (a state) of the following system of motion equations (of the corresponding dynamical system)

$$
\dot{x}_{n}=f_{n}(x), 1 \leq n \leq N
$$

we can consider the question of stability of the solution, the existence of the solutions of the type $x_{n}=x_{0 n}+g_{n}$, for small values of $g_{n}$. If there are solutions with rising $g_{n}$, of the corresponding motion equations

$$
\begin{aligned}
& \dot{g}_{n}=\beta_{n}(g), \beta_{n}(g)=f_{n}\left(x_{0}+g\right)-f_{n}\left(x_{0}\right) \\
& =\beta_{1 n m} g_{m}+\beta_{2 n m k} g_{m} g_{k}+\ldots, \beta_{k n \ldots m}=f^{(n \ldots m)}\left(x_{0}\right)
\end{aligned}
$$

we say that the solution $x_{0 n}$ is not stable.

The linear approximation, we transform into diagonal form,

$$
\begin{aligned}
& \dot{g}_{n}=\beta_{1 n m} g_{m}, h_{n}=A_{n m} g_{m}, \\
& \dot{h}_{n}=\lambda_{n} h_{n}, \lambda_{n} \delta_{n m}=\left(A \beta_{1} A^{-1}\right)_{n m},
\end{aligned}
$$

if all of the $\lambda_{n}$ are purely imaginary $\lambda_{n}=i \omega_{n}$, we have stable solution (in the linear approximation): small deviations remain small. If real parts of all $\lambda_{n}$ are negative, we have asymptotic stability: deviations decrease. If some $\lambda_{n}$ are zero, we have undefined case. In regular case, when the matrix $\beta_{1}$ has inverse, by reparametrization trick we can construct the formal solution of the nonlinear equation for $g_{n}$, and try to investigate its convergence properties.

\section{Nambu Dynamics}

Nabu - Babylonian God of Wisdom and Writing.

The Hamiltonian mechanics (HM) is in the fundamentals of mathematical description of the physical theories [Faddeev, Takhtajan, 1990]. But HM is in a sense blind; e.g., it does not make a difference between two opposites: the ergodic Hamiltonian systems (with just one integral of motion) [Sinai, 1993] and (super)integrable Hamiltonian systems (with maximal number of the integrals of motion).

Nabu mechanics (NM) [Nambu, 1973, Whittaker, 1927] is a proper generalization of the HM, which makes the difference between dynamical systems with different numbers of integrals of motion explicit (see, e.g. Makhaldiani, 2007]). 
In the canonical formulation, the equations of motion of a physical system are defined via a Poisson bracket and a Hamiltonian, [Arnold, 1978]. In Nambu's formulation, the Poisson bracket is replaced by the Nambu bracket with $n+1, n \geq 1$, slots. For $n=1$, we have the canonical formalism with one Hamiltonian. For $n \geq 2$, we have Nambu-Poisson formalism, with $n$ Hamiltonians, [Nambu, 1973], [Whittaker, 1927].

\subsection{Nambu Dynamics, System of Three Vortexes}

The system of $N$ vortexes can be described by the following system of differential equations, [Aref, 1983, Meleshko,Konstantinov, 1993]

$$
\dot{z}_{n}=i \sum_{m \neq n}^{N} \frac{\gamma_{m}}{z_{n}^{*}-z_{m}^{*}}, \quad 1 \leq n \leq N,
$$

where $z_{n}=x_{n}+i y_{n}$ are complex coordinate of the centre of n-th vortex, for $N=3$, and the quantities

$$
\begin{aligned}
& u_{1}=\ln \left|z_{2}-z_{3}\right|^{2}, \\
& u_{2}=\ln \left|z_{3}-z_{1}\right|^{2}, \\
& u_{3}=\ln \left|z_{1}-z_{2}\right|^{2}
\end{aligned}
$$

reduce to the following system

$$
\begin{aligned}
& \dot{u}_{1}=\gamma_{1}\left(e^{u_{2}}-e^{u_{3}}\right), \\
& \dot{u}_{2}=\gamma_{2}\left(e^{u_{3}}-e^{u_{1}}\right), \\
& \dot{u}_{3}=\gamma_{3}\left(e^{u_{1}}-e^{u_{2}}\right),
\end{aligned}
$$

The system ([16.3) has two integrals of motion

$$
H_{1}=\sum_{i=1}^{3} \frac{e^{u_{i}}}{\gamma_{i}}, H_{2}=\sum_{i=1}^{3} \frac{u_{i}}{\gamma_{i}}
$$

and can be presented in the Nambu-Poisson form, [Makhaldiani, 1997,2]

$$
\dot{u}_{i}=\omega_{i j k} \frac{\partial H_{1}}{\partial u_{j}} \frac{\partial H_{2}}{\partial u_{k}}=\left\{x_{i}, H_{1}, H_{2}\right\}=\omega_{i j k} \frac{e^{u_{j}}}{\gamma_{j}} \frac{1}{\gamma_{k}},
$$

where

$$
\omega_{i j k}=\varepsilon_{i j k} \rho, \rho=\gamma_{1} \gamma_{2} \gamma_{3}
$$

and the Nambu-Poisson bracket of the functions $A, B, C$ on the three-dimensional phase space is

$$
\{A, B, C\}=\omega_{i j k} \frac{\partial A}{\partial u_{i}} \frac{\partial B}{\partial u_{j}} \frac{\partial C}{\partial u_{k}}
$$

This system is superintegrable: for $N=3$ degrees of freedom, we have maximal number of the integrals of motion $N-1=2$. 


\subsection{Toward the Finite Unified Field Theory}

The reduction of the dimensionless couplings in GUTs is achieved by searching for RD integrals of motion-renormdynamic invariant (RDI) relations among them holding beyond the unification scale. Finiteness results from the fact that there exist RDI relations among dimensional couplings that guarantee the vanishing of all beta-functions in certain GUTs even to all orders. In this case the number of the independent motion integrals $\mathrm{N}$ is equal to the number of the coupling constants. Note that in superintegrable dynamical systems the number of the integrals is $\leq N-1$, so the RD of the finite field theories is trivial, coupling constants do not run, they have fixed values, the renormdynamics is more than superintegrable, it is hyperintegrable. Developments in the soft supersymmetry breaking sector of GUTs and FUTs lead to exact RDI relations, i.e. reduction of couplings, in this dimensionful sector of the theory, too. Based on the above theoretical framework phenomenologically consistent FUTs have been constructed. The main goal expected from a unified description of interactions by the particle physics community is to understand the present day large number of free parameters of the SM in terms of a few fundamental ones. In other words, to achieve reduction of couplings at a more fundamental level.

\subsection{Nambu Dynamics, Extended Quantum Mechanics}

As an example of the infinite dimensional Nambu-Poisson dynamics, let me conside the following extension of Schrödinger quantum mechanics [Makhaldiani, 2000]

$$
\begin{aligned}
& i V_{t}=\Delta V-\frac{V^{2}}{2}, \\
& i \psi_{t}=-\Delta \psi+V \psi
\end{aligned}
$$

An interesting solution to the equation for the potential (116.5) is

$$
V=\frac{4(4-d)}{r^{2}}
$$

where $d$ is the dimension of the spase. In the case of $d=1$, we have the potential of conformal quantum mechanics.

The variational formulation of the extended quantum theory, is given by the following Lagrangian

$$
L=\left(i V_{t}-\Delta V+\frac{1}{2} V^{2}\right) \psi
$$

The momentum variables are

$$
P_{v}=\frac{\partial L}{\partial V_{t}}=i \psi, P_{\psi}=0
$$

As Hamiltonians of the Nambu-theoretic formulation, we take the following integrals of motion

$$
\begin{aligned}
& H_{1}=\int d^{d} x\left(\Delta V-\frac{1}{2} V^{2}\right) \psi, \\
& H_{2}=\int d^{d} x\left(P_{v}-i \psi\right),
\end{aligned}
$$




$$
H_{3}=\int d^{d} x P_{\psi}
$$

We invent unifying vector notation, $\phi=\left(\phi_{1}, \phi_{2}, \phi_{3}, \phi_{4}\right)=\left(\psi, P_{\psi}, V, P_{v}\right)$. Then it may be verified that the equations of the extended quantum theory can be put in the following Nambu-theoretic form

$$
\phi_{t}(x)=\left\{\phi(x), H_{1}, H_{2}, H_{3}\right\}
$$

where the bracket is defined as

$$
\begin{aligned}
& \left\{A_{1}, A_{2}, A_{3}, A_{4}\right\}=i \varepsilon_{i j k l} \int \frac{\delta A_{1}}{\delta \phi_{i}(y)} \frac{\delta A_{2}}{\delta \phi_{j}(y)} \frac{\delta A_{3}}{\delta \phi_{k}(y)} \frac{\delta A_{4}}{\delta \phi_{l}(y)} d y \\
& =i \int \frac{\delta\left(A_{1}, A_{2}, A_{3}, A_{4}\right)}{\delta\left(\phi_{1}(y), \phi_{2}(y), \phi_{3}(y), \phi_{4}(y)\right)} d y=i \operatorname{det}\left(\frac{\delta A_{k}}{\delta \phi_{l}}\right) .
\end{aligned}
$$

\subsection{Nambu Dynamics, $M$ theory}

The basic building blocks of $\mathrm{M}$ theory are membranes and M5-branes. Membranes are fundamental objects carrying electric charges with respect to the 3-form $C$-field, and $M 5$-branes are magnetic solitons. The Nambu-Poisson 3-algebras appear as gauge symmetries of superconformal Chern-Simons nonabelian theories in $2+1$ dimensions with the maximum allowed number of $N=8$ linear supersymmetries.

The Bagger and Lambert [Bagger, Lambert, 2007] and, Gustavsson [Gustavsson, 2007] (BLG) model is based on a 3-algebra,

$$
\left[T^{a}, T^{b}, T^{c}\right]=f_{d}^{a b c} T^{d}
$$

where $T^{a}$, are generators and $f_{a b c d}$ is a fully anti-symmetric tensor. Given this algebra, a maximally supersymmetric Chern-Simons lagrangian is:

$$
\begin{aligned}
L= & L_{C S}+L_{\text {matter }}, \\
L_{C S} & =\frac{1}{2} \varepsilon^{\mu v \lambda}\left(f_{a b c d} A_{\mu}^{a b} \partial_{v} A_{\lambda}^{c d}+\frac{2}{3} f_{c d a g} f_{e f b}^{g} A_{\mu}^{a b} A_{v}^{c d} A_{\lambda}^{e f}\right), \\
& L_{\text {matter }}=\frac{1}{2} B_{\mu}^{I a} B_{a}^{\mu I}-B_{\mu}^{I a} D^{\mu} X_{a}^{I} \\
& +\frac{i}{2} \bar{\psi}^{a} \Gamma^{\mu} D_{\mu} \psi_{a}+\frac{i}{4} \bar{\psi}^{b} \Gamma_{I J} x_{c}^{I} x_{d}^{J} \psi_{a} f^{a b c d} \\
& -\frac{1}{12} \operatorname{tr}\left(\left[X^{I}, X^{J}, X^{K}\right]\left[X^{I}, X^{J}, X^{K}\right]\right), I=1,2, \ldots, 8
\end{aligned}
$$

where $A_{\mu}^{a b}$ is gauge boson, $\psi^{a}$ and $X^{I}=X_{a}^{I} T^{a}$ matter fields. If $a=1,2,3,4$, then we can obtain an $S O(4)$ gauge symmetry by choosing $f_{a b c d}=f \varepsilon_{a b c d}, f$ being a constant. It turns out to be the only case that gives a gauge theory with manifest unitarity and $N=8$ supersymmetry.

The action has the first order form so we can use the formalism of the first section. The motion equations for the gauge fields

$$
f_{a b c d}^{n m} \dot{A}_{m}^{c d}(t, x)=\frac{\delta H}{\delta A_{n}^{a b}(t, x)}, f_{a b c d}^{n m}=\varepsilon^{n m} f_{a b c d}
$$


take canonical form

$$
\begin{aligned}
& \dot{A}_{n}^{a b}=f_{n m}^{a b c d} \frac{\delta H}{\delta A_{m}^{c d}}=\left\{A_{n}^{a b}, A_{m}^{c d}\right\} \frac{\delta H}{\delta A_{m}^{c d}}=\left\{A_{n}^{a b}, H\right\} \\
& \left\{A_{n}^{a b}(t, x), A_{m}^{c d}(t, y)\right\}=\varepsilon_{n m} f^{a b c d} \delta^{(2)}(x-y)
\end{aligned}
$$

\subsection{Nambu-Poisson Dynamics of an Extended Particle With Spin in an Accelerator}

The quasi-classical description of the motion of a relativistic (nonradiating) point particle with spin in accelerators and storage rings includes the equations of orbit motion

$$
\begin{aligned}
& \dot{x}_{n}=f_{n}(x), f_{n}(x)=\varepsilon_{n m} \partial_{m} H, n, m=1,2, \ldots, 6 ; \\
& x_{n}=q_{n}, x_{n+3}=p_{n}, \varepsilon_{n, n+3}=1, n=1,2,3 \\
& H=e \Phi+c \sqrt{\wp^{2}+m^{2} c^{2}}, \wp_{n}=p_{n}-\frac{e}{c} A_{n}
\end{aligned}
$$

and Thomas-BMT equations [Tomas, 1927, Bargmann, Michel,Telegdi, 1959] of classical spin motion

$$
\begin{gathered}
\dot{s}_{n}=\varepsilon_{n m k} \Omega_{m} s_{k}=\left\{H_{1}, H_{2}, s_{n}\right\}, H_{1}=\Omega \cdot s, H_{2}=s^{2}, \\
\{A, B, C\}=\varepsilon_{n m k} \partial_{n} A \partial_{m} B \partial_{k} C \\
\Omega_{n}=\frac{-e}{m \gamma c}\left((1+k \gamma) B_{n}-k \frac{(B \cdot \wp) \wp_{n}}{m^{2} c^{2}(1+\gamma)}\right. \\
\left.+\frac{1+k(1+\gamma)}{m c(1+\gamma)} \varepsilon_{n m k} E_{m} \wp_{k}\right)
\end{gathered}
$$

where, parameters $e$ and $m$ are the charge and the rest mass of the particle, $c$ is the velocity of light, $k=(g-2) / 2$ quantifies the anomalous spin $g$ factor, $\gamma$ is the Lorentz factor, $p_{n}$ are components of the kinetic momentum vector, $E_{n}$ and $B_{n}$ are the electric and magnetic fields, and $A_{n}$ and $\Phi$ are the vector and scalar potentials;

$$
\begin{aligned}
& B_{n}=\varepsilon_{n m k} \partial_{m} A_{k}, E_{n}=-\partial_{n} \Phi-\frac{1}{c} \dot{A}_{n}, \\
& \gamma=\frac{H-e \Phi}{m c^{2}}=\sqrt{1+\frac{\wp^{2}}{m^{2} c^{2}}}
\end{aligned}
$$

The spin motion equations we put in the Nambu-Poisson form. Hamiltonization of this dynamical system according to the general approach of the previous sections we will put in the ground of the optimal control theory of the accelerator.

\subsection{Hamiltonian Extension of the Spinning Particle Dynamics}

The general method of Hamiltonization of the dynamical systems we can use also in the spinning particle case. Let us invent unified configuration space $q=(x, p, s), x_{n}=q_{n}, p_{n}=q_{n+3}, s_{n}=$ $q_{n+6}, n=1,2,3$; extended phase space, $\left(q_{n}, \psi_{n}\right)$ and hamiltonian

$$
H=H(q, \psi)=v_{n} \psi_{n}, n=1,2, \ldots 9 ;
$$


motion equations

$$
\begin{aligned}
& \dot{q}_{n}=v_{n}(q), \\
& \dot{\psi}_{n}=-\frac{\partial v_{m}}{\partial q_{n}} \psi_{m}
\end{aligned}
$$

where the velocities $v_{n}$ depends on external fields as in previous section as control parameters which can be determined according to the optimal control criterium.

\subsection{Nonrelativistic charged point particle with radiation friction}

Nonrelativistic motion equation of the point charged particle including effect of the radiation self-friction is [Ginzburg, 1979]

$$
m \frac{d^{2} x_{n}}{d t^{2}}=F_{n}+\frac{2 e^{2}}{3 c^{3}} \frac{d^{3} x_{n}}{d t^{3}}, n=1,2,3
$$

where the second therm in r.h.s is the $\mathrm{f}(\mathrm{r})$ iction force and vector $\mathrm{F}$ is external force. Not that if we invent an effective mass and charge as $m_{\star}=2 / 3 m$ and $e_{\star}=2 / 3 e$ the equation receives an universal form

$$
m_{\star} \frac{d^{2} x}{d t^{2}}=F_{\star}+\frac{e_{\star}^{2}}{c^{3}} \frac{d^{3} x_{n}}{d t^{3}}, F_{\star}=\frac{2}{3} F
$$

When the external force is electromagnetic-the Lorentz force,

$$
F=e\left(E+\frac{1}{c}[V \times B]\right)
$$

and invention of the effective charge is consistent, $F_{\star}(e)=F\left(e_{\star}\right)$.

\section{Discrete Dynamical Systems and Quanputers}

Computers are physical devices and their behavior is determined by physical laws. The Quantum Computations [Benenti, Casati, Strini, 2004, Nielsen, Chuang, 2000], Quantum Computing, Quanputing [Makhaldiani, 2007.2], is a new interdisciplinary field of research, which benefits from the contributions of physicists, computer scientists, mathematicians, chemists and engineers.

Contemporary digital computer and its logical elements can be considered as a spatial type of discrete dynamical systems [Makhaldiani, 200]]

$$
S_{n}(k+1)=\Phi_{n}(S(k))
$$

where

$$
S_{n}(k), \quad 1 \leq n \leq N(k),
$$

is the state vector of the system at the discrete time step $k$. Vector $S$ may describe the state and $\Phi$ transition rule of some Cellular Automata [Toffoli, Margolus, 1987]. The systems of the type (II7. I) appears in applied mathematics as an explicit finite difference scheme approximation of the equations of the physics [Samarskii, Gulin, 1989]. 
Definition: We assume that the system ([7]) is time-reversible if we can define the reverse dynamical system

$$
S_{n}(k)=\Phi_{n}^{-1}(S(k+1))
$$

In this case the following matrix

$$
M_{n m}=\frac{\partial \Phi_{n}(S(k))}{\partial S_{m}(k)}
$$

is regular, i.e. has an inverse. If the matrix is not regular, this is the case, for example, when $N(k+$ $1) \neq N(k)$, we have an irreversible dynamical system (usual digital computers and/or corresponding irreversible gates).

Let us consider an extension of the dynamical system (ㅍ.]) given by the following action function

$$
A=\sum_{k n} l_{n}(k)\left(S_{n}(k+1)-\Phi_{n}(S(k))\right)
$$

and corresponding motion equations

$$
\begin{aligned}
& S_{n}(k+1)=\Phi_{n}(S(k))=\frac{\partial H}{\partial l_{n}(k)}, \\
& l_{n}(k-1)=l_{m}(k) \frac{\partial \Phi_{m}(S(k))}{\partial S_{n}(k)}=l_{m}(k) M_{m n}(S(k))=\frac{\partial H}{\partial S_{n}(k)},
\end{aligned}
$$

where

$$
H=\sum_{k n} l_{n}(k) \Phi_{n}(S(k))
$$

is discrete Hamiltonian. In the regular case, we put the system ([17.6) in an explicit form

$$
\begin{aligned}
& S_{n}(k+1)=\Phi_{n}(S(k)), \\
& l_{n}(k+1)=l_{m}(k) M_{m n}^{-1}(S(k+1)) .
\end{aligned}
$$

From this system it is obvious that, when the initial value $l_{n}\left(k_{0}\right)$ is given, the evolution of the vector $l(k)$ is defined by evolution of the state vector $S(k)$. The equation of motion for $l_{n}(k)-$ Elenka is linear and has an important property that a linear superpositions of the solutions are also solutions.

Statement: Any time-reversible dynamical system (e.g. a time-reversible computer) can be extended by corresponding linear dynamical system (quantum - like processor) which is controlled by the dynamical system and has a huge computational power, [Makhaldiani, 2001, Makhaldiani, 2002, Makhaldiani, 2007.2, Makhaldiani, 2011.2].

\section{1 (de)Coherence Criterion}

For motion equations ([T.6) in the continual approximation, we have

$$
S_{n}(k+1)=x_{n}\left(t_{k}+\tau\right)=x_{n}\left(t_{k}\right)+\dot{x}_{n}\left(t_{k}\right) \tau+O\left(\tau^{2}\right),
$$




$$
\begin{aligned}
& \dot{x}_{n}\left(t_{k}\right)=v_{n}\left(x\left(t_{k}\right)\right)+O(\tau), t_{k}=k \tau, \\
& v_{n}\left(x\left(t_{k}\right)\right)=\left(\Phi_{n}\left(x\left(t_{k}\right)\right)-x_{n}\left(t_{k}\right)\right) / \tau ; \\
& M_{m n}\left(x\left(t_{k}\right)\right)=\delta_{m n}+\tau \frac{\partial v_{m}\left(x\left(t_{k}\right)\right)}{\partial x_{n}\left(t_{k}\right)} .
\end{aligned}
$$

(de)Coherence criterion: the system is reversible, the linear (quantum, coherent, soul) subsystem exists, when the matrix $M$ is regular,

$$
\operatorname{det} M=1+\tau \sum_{n} \frac{\partial v_{n}}{\partial x_{n}}+O\left(\tau^{2}\right) \neq 0
$$

For the Nambu - Poisson dynamical systems (see e.g. [Makhaldiani, 2007])

$$
\begin{aligned}
& v_{n}(x)=\varepsilon_{n m_{1} m_{2} \ldots m_{p}} \frac{\partial H_{1}}{\partial x_{m_{1}}} \frac{\partial H_{2}}{\partial x_{m_{2}}} \ldots \frac{\partial H_{p}}{\partial x_{m_{p}}}, p=1,2,3, \ldots, N-1, \\
& \sum_{n} \frac{\partial v_{n}}{\partial x_{n}} \equiv d i v v=0 .
\end{aligned}
$$

\section{Construction of the Reversible Discrete Dynamical Systems}

Let me motivate an idea of construction of the reversible dynamical systems by simple example from field theory. There are renormalizable models of scalar field theory of the form (see, e.g. [Makhaldiani, 1980])

$$
L=\frac{1}{2}\left(\partial_{\mu} \varphi \partial^{\mu} \varphi-m^{2} \varphi^{2}\right)-g \varphi^{n}
$$

with the constraint

$$
n=\frac{2 d}{d-2}
$$

where $d$ is dimension of the space-time and $n$ is degree of nonlinearity. It is interesting that if we define $d$ as a function of $n$, we find

$$
d=\frac{2 n}{n-2}
$$

the same function!

Thing is that, the constraint can be put in the symmetric implicit form [Makhaldiani, 1980]

$$
\frac{1}{n}+\frac{1}{d}=\frac{1}{2}
$$

\subsection{Generalization of the Idea}

Now it is natural to consider the following symmetric function

$$
f(y)+f(x)=c
$$

and define its solution

$$
y=f^{-1}(c-f(x)) .
$$


This is the general method, that we will use in the following construction of the reversible dynamical systems. In the simplest case,

$$
f(x)=x
$$

we take

$$
y=S(k+1), x=S(k-1), c=\tilde{\Phi}(S(k))
$$

and define our reversible dynamical system from the following symmetric, implicit form (see also [Toffoli, Margolus, 1987])

$$
S(k+1)+S(k-1)=\tilde{\Phi}(S(k))
$$

explicit form of which is

$$
\begin{aligned}
S(k+1) & =\Phi(S(k), S(k-1)) \\
& =\tilde{\Phi}(S(k))-S(k-1) .
\end{aligned}
$$

This dynamical system defines given state vector by previous two state vectors. We have reversible dynamical system on the time lattice with time steps of two units,

$$
\begin{aligned}
& S(k+2,2)=\Phi(S(k, 2)), \\
& S(k+2,2) \equiv(S(k+2), S(k+1)), \\
& S(k, 2) \equiv(S(k), S(k-1))) .
\end{aligned}
$$

\subsection{Internal, Spin, Degrees of Freedom}

Starting from a general discrete dynamical system, we obtained reversible dynamical system with internal(spin,bit) degrees of freedom

$$
\begin{aligned}
S_{n s}(k+2) & \equiv\left(\begin{array}{c}
S_{n}(k+2) \\
S_{n}(k+1)
\end{array}\right)=\left(\begin{array}{c}
\left.\Phi_{n}(\Phi(S(k))-S(k-1))-S(k)\right) \\
\Phi_{n}(S(k))-S_{n}(k-1)
\end{array}\right) \\
& \equiv \Phi_{n s}(S(k)), \quad s=1,2
\end{aligned}
$$

where

$$
S(k) \equiv\left(S_{n s}(k)\right), S_{n 1}(k) \equiv S_{n}(k), S_{n 2}(k) \equiv S_{n}(k-1)
$$

For the extended system we have the following action

$$
A=\sum_{k n s} l_{n s}(k)\left(S_{n s}(k+2)-\Phi_{n s}(S(k))\right)
$$

and corresponding motion equations

$$
\begin{aligned}
& S_{n s}(k+2)=\Phi_{n s}(S(k))=\frac{\partial H}{\partial l_{n s}(k)}, \\
& l_{n s}(k-2)=l_{m t}(k) \frac{\partial \Phi_{m t}(S(k))}{\partial S_{n s}(k)}
\end{aligned}
$$




$$
=l_{m t}(k) M_{m t n s}(S(k))=\frac{\partial H}{\partial S_{n s}(k)},
$$

By construction, we have the following reversible dynamical system

$$
\begin{aligned}
& S_{n s}(k+2)=\Phi_{n s}(S(k)), \\
& l_{n s}(k+2)=l_{m t}(k) M_{m t n s}^{-1}(S(k+2)),
\end{aligned}
$$

with classical $S_{n s}$ and quantum $l_{n s}($ in the external, background S) string bit dynamics.

\section{3 p-Point Cluster and Higher Spin States Reversible Dynamics, or Put String Dynamics}

We can also consider p-point generalization of the previous structure,

$$
\begin{aligned}
& f_{p}(S(k+p))+f_{p-1}(S(k+p-1))+\ldots+f_{1}(S(k+1)) \\
& +f_{1}(S(k-1))+\ldots+f_{p}(S(k-p))=\tilde{\Phi}(S(k)), \\
& S(k+p)=\Phi(S(k), S(k+p-1), \ldots, S(k-p)) \\
& \equiv f_{p}^{-1}\left(\tilde{\Phi}(S(k))-f_{p-1}(S(k+p-1))-\ldots-f_{p}(S(k-p))\right)
\end{aligned}
$$

and corresponding reversible p-oint cluster dynamical system

$$
\begin{aligned}
& S(k+p, p) \equiv \Phi(S(k, p)), \\
& S(k+p, p) \equiv(S(k+p), S(k+p-1), \ldots, S(k+1)), \\
& S(k, p) \equiv(S(k), S(k-1), \ldots, S(k-p+1)), S(k, 1)=S(k) .
\end{aligned}
$$

So we have general method of construction of the reversible dynamical systems on the time (tame) scale $p$. The method of linear extension of the reversible dynamical systems (see [Makhaldiani, 200]] and previous section) defines corresponding Quanputers,

$$
\begin{aligned}
& S_{n s}(k+p)=\Phi_{n s}(S(k)), \\
& l_{n s}(k+p)=l_{m t}(k) M_{m t n s}^{-1}(S(k+p)),
\end{aligned}
$$

This case the quantum state function $l_{n s}, s=1,2, \ldots p$ will describes the state with spin $(p-$ 1) $/ 2$.

Note that, in this formalism for reversible dynamics minimal value of the spin is $1 / 2$. There is not a place for a scalar dynamics, or the scalar dynamics is not reversible. In the Standard model (SM) of particle physics, [Beringer et al, 2012], all of the fundamental particles, leptons, quarks and gauge bosons have spin. Only scalar particles of the SM are the Higgs bosons. Perhaps the scalar particles are composed systems or quasiparticles like phonon, or Higgs dynamics is not reversible (a mechanism for 'time arrow').

\section{Classically Nonalgorithmic but Quanputally Solvable Problems}

\subsection{Diophantine equations}

Diophantine equations (DE)

$$
D(n)=D\left(n_{1}, \ldots, n_{m}\right)=0,
$$

are those equations that have integer solutions. In particular, they may be polynomials with integer coefficients. 


\subsection{Quantization of DE}

For quantization of DE, (QDE), consider monomials

$$
x^{n} \doteq x_{1}^{n_{1}} \ldots x_{m}^{n_{m}},
$$

than, for solutions of DE

$$
0=D(n) x^{n}=D(\delta) x^{n}=0
$$

where

$$
\begin{aligned}
& D(\boldsymbol{\delta}) \doteq D\left(\delta_{1}, \ldots, \delta_{n}\right), \delta_{k}=x_{k} \partial_{x_{k}}, k=1, \ldots, m, \\
& \delta_{k} x^{n}=n_{k} x^{n}
\end{aligned}
$$

so, existence of the solution of DE is equivalent to existence of the eigenstate-monomials of the QED.

\subsection{Quantum algorithm of solution of the QDE}

For quanputing of the problem we introduce the following Hamiltonian operator,

$$
H(\delta)=D(\delta)^{2}, H(\delta) x^{n}=H(n) x^{n}, H(n) \geq 0 .
$$

Quanputing may algorithmically define if $H(n)=0$ for some $n$, so, may algorithmically solve DE.

\section{How to work on quanputers}

We explain the idea of programming on quanputers on the example of extended quantum mechanics. We extend the motion equation for the potential and corresponding action by introduction of the external source $J$,

$$
\begin{aligned}
& L=\left(i V_{t}-\Delta V+\frac{1}{2} V^{2}-J\right) \psi, \\
& i V_{t}=\Delta V-\frac{1}{2} V^{2}+J, \\
& i \psi_{t}=-\Delta \psi+V \psi .
\end{aligned}
$$

Different sources define different potentials-programs which controls dynamics of the linear part of quanputers, $\psi$. Having an algorithm for quanputer we know corresponding $V(t, x)$. With this potential we calculate corresponding source $J$. Note that $J$ is the source therm in action (Lagrangian) for $\psi$ and enter as a source term in the motion equation of $V$.

\subsection{How to make (simple) programs}

The trivial case $J=0$, corresponds to a vacation of the quanputer. As a next simplest case let us consider $J=m^{2} / 2=$ const. From the motion equation, we obtain $V= \pm m$. So, we have two level quantum system, for the linear, quantum-like, $\psi$-subsystem. Then, we may consider simplest time-dependent source $J=m^{2} e^{i \omega t}$. Note that the source do not define the potential uniquely. 


\subsection{Extended Quantum Dynamics in One Dimensional Space}

In the case of one dimensional space, $D=1$ from the motion equation for the potential

$$
i V_{t}=V_{x x}-\frac{1}{2} V^{2}+J
$$

we obtain

$$
i \bar{\psi}_{t}=\bar{\psi}_{x x}-V \bar{\psi}+j, \bar{\psi}=V_{x}, j=J_{x}
$$

so, when $V$ is the solution for the motion equation for $V, \bar{\psi}=V_{x}$ is the solution for the motion equation for $\bar{\psi}$, corresponding equation for $\psi$,

$$
i \psi_{t}=-\Delta \psi+\bar{V} \psi-\bar{j}
$$

\subsection{Static case}

For the potential, we have

$$
V^{\prime \prime}-\frac{1}{2} V^{2}+J=0
$$

In the case of trivial source, $J=0$, the vanishing in the infinity solution is

$$
V(x)=\frac{12}{x^{2}}
$$

In the case of the constant source,

$$
\int \frac{d V}{\sqrt{V^{3} / 3-2 J V}}=2 \sqrt{3} \int \frac{d V}{\sqrt{4 V^{3}-24 J V}}= \pm x
$$

\section{Transcendental numbers and Hilbert's seventh problem}

In mathematics, a transcendental number is a (possibly complex) number that is not algebraic, i.e. it is not a root of a non-zero polynomial equation with rational coefficients. The most prominent examples of transcendental numbers are $\pi$ and $e$. Though only a few classes of transcendental numbers are known (in part because it can be extremely difficult to show that a given number is transcendental), transcendental numbers are not rare. Indeed, almost all real and complex numbers are transcendental, since the algebraic numbers are countable while the sets of real and complex numbers are both uncountable. All real transcendental numbers are irrational, since all rational numbers are algebraic. The converse is not true: not all irrational numbers are transcendental; e.g., the square root of 2 is irrational but not a transcendental number. The name "transcendental" comes from Leibniz in his 1682 paper where he proved that $\sin (x)$ is not an algebraic function of $x$. Euler was probably the first person to define transcendental numbers in the modern sense. Johann Heinrich Lambert conjectured that $e$ and $\pi$ were both transcendental numbers in his 1761 paper proving the number $\pi$ is irrational. In 1874 , Georg Cantor proved that the algebraic numbers are countable and the real numbers are uncountable. In 1882, Ferdinand von Lindemann published a 
proof that the number $\pi$ is transcendental. He first showed that $e$ to any nonzero algebraic power is transcendental, and since $e^{i \pi}=-1$ is algebraic ( Euler's identity), $i \pi$ and therefore $\pi$ must be transcendental. In 1900, David Hilbert posed an influential question about transcendental numbers, Hilbert's seventh problem: If $a$ is an algebraic number, that is not zero or one, and $b$ is an irrational algebraic number, is $a^{b}$ necessarily transcendental? The affirmative answer was provided in 1934 by the Gelfond-Schneider theorem. This work was extended by Alan Baker in the 1960s in his work on lower bounds for linear forms in any number of logarithms (of algebraic numbers).

Numbers proven to be transcendental includes e.g. the Gelfond's constant $=e^{\pi}$ and Chaitin's constant (since it is a non-computable number) $\Omega$.

Numbers which have yet to be proven to be either transcendental or algebraic includes:

The Euler-Mascheroni constant $\gamma$ (which has not even been proven to be irrational).

Catalan's constant, also not known to be irrational.

Apéry's constant, $\zeta(3)$ (which Apéry proved is irrational)

The Riemann zeta function at other odd integers, $\zeta(5), \zeta(7), \ldots$ (not known to be irrational.)

The Feigenbaum constants, $\delta$ and $\alpha$.

The non-computable numbers are a strict subset of the transcendental numbers.

If a given number $a$ is algebraic, it is a solution of a polynomial equation: $P(a)=0$. In the operator form it is equivalent to the statement: $P(\delta) x^{a}=P(a) x^{a}=0$. For the Hamiltonian of the quantum algorithm we take $H=P(\delta)^{2}$.

\section{Discrete invertible dynamical systems}

Let us consider the stationary Schrodinger equation

$$
\left(-\frac{\hbar^{2}}{2 m} \triangle+V\right) \psi=E \psi \Rightarrow \triangle \psi=a \psi, a=\frac{2 m}{\hbar^{2}}(V-E)
$$

for one dimensional case, corresponding discrete (approximation) equation is

$$
\begin{aligned}
& \psi_{n+1}=V_{n} \psi_{n}-\psi_{n-1}, \\
& V_{n}=2+a_{n}, a_{n}=\frac{2 m}{\hbar^{2}}\left(V\left(x_{n}\right)-E\right), x_{n}=n h, \psi_{n}=\psi\left(x_{n}\right)
\end{aligned}
$$

from which we obtain

$$
\begin{aligned}
& \Phi_{n+1}=A \Phi_{n}, \\
& \Phi_{n+1}=\left(\begin{array}{l}
\psi_{n+1} \\
\psi_{n+2}
\end{array}\right), \Phi_{n}=\left(\begin{array}{c}
\psi_{n-1} \\
\psi_{n}
\end{array}\right), \\
& A=\left(\begin{array}{cc}
-1, & V_{n} \\
-V_{n+1}, & V_{n} V_{n+1}-1
\end{array}\right), \operatorname{det} A=1, A^{-1}=\left(\begin{array}{cc}
V_{n} V_{n+1}-1, & -V_{n} \\
V_{n+1}, & -1
\end{array}\right), \\
& A=\left(\begin{array}{cc}
a & b \\
c & d
\end{array}\right) \\
& A^{-1}=\left(\begin{array}{cc}
d & -b \\
-c & a
\end{array}\right) / \operatorname{det} A
\end{aligned}
$$


Note that, the potential term $V_{n}$ may depend on the wave function $\psi_{n}$, so we can consider also nonlinear invertible dynamical systems.

\section{1 q-Hermitian Polynomials}

q-Hermitian Polynomials (qHp) are defined by the recurrent relation [Gasper, Rahman, 1990]

$$
P_{n+1}=x P_{n}(x)-u_{n} P_{n-1}, u_{n}=1-q^{n}
$$

from which we obtain

$$
\begin{aligned}
& \Phi_{n+1}=A \Phi_{n}, \\
& \Phi_{n+1}=\left(\begin{array}{c}
P_{n+1} \\
P_{n+2}
\end{array}\right), \Phi_{n}=\left(\begin{array}{c}
P_{n-1} \\
P_{n}
\end{array}\right), \\
& A=\left(\begin{array}{cc}
-u_{n}, & x \\
-x u_{n}, x^{2}-u_{n+1}
\end{array}\right), \operatorname{det} A=u_{n} u_{n+1}=(1-q)^{2}[n][n+1], \\
& A^{-1}=\left(\begin{array}{cc}
x^{2}-u_{n+1}, & -x \\
x u_{n}, & -u_{n}
\end{array}\right) / \operatorname{det} A, A=\left(\begin{array}{ll}
a & b \\
c & d
\end{array}\right), A^{-1}=\left(\begin{array}{cc}
d & -b \\
-c & a
\end{array}\right) / \operatorname{det} A
\end{aligned}
$$

Note that, for $q=1, u_{n}=0 \Rightarrow P_{n}=c x^{n}$. For $q=0, u_{n}=1$ and we obtain symmetric invertible dynamical system

$$
P_{n+1}+P_{n-1}=x P_{n}(x)
$$

With the solution

$$
P_{n}=c a^{n}, a+a^{-1}=x \Rightarrow a=\left(x \pm \sqrt{x^{2}-4}\right) / 2
$$

If we are interested for real valued solutions, we have restriction $|x| \geq 0$, we have the minimal value for $|x|$, a fundamental length. For $q=-1, u_{2 k}=0, u_{2 k+1}=2$

\subsection{Fibonacci, Hindu-Arabic numerals and time-reversible discrete dynamical systems}

Fibonacci, recognizing that arithmetic with Hindu-Arabic numerals is simpler and more efficient than with Roman numerals, travelled throughout the Mediterranean world to study under the leading Arab mathematicians of the time. He returned from his travels around 1200, and in 1202, the 32-year-old recorded what he had learned in Liber Abaci (Book of Abacus or Book of Calculation), and thereby popularized Hindu-Arabic numerals in Europe.

In the Fibonacci sequence of numbers, each number is the sum of the previous two numbers,

$$
z_{n+1}=z_{n}+z_{n-1}
$$

from which we obtain

$$
\begin{aligned}
& \Phi_{n+1}=A \Phi_{n}, \\
& \Phi_{n+1}=\left(\begin{array}{c}
z_{n+1} \\
z_{n+2}
\end{array}\right), \Phi_{n}=\left(\begin{array}{c}
z_{n-1} \\
z_{n}
\end{array}\right),
\end{aligned}
$$




$$
\begin{aligned}
& A=\left(\begin{array}{ll}
1 & 1 \\
1 & 2
\end{array}\right), \operatorname{det} A=1, A^{-1}=\left(\begin{array}{cc}
2 & -1 \\
-1 & 1
\end{array}\right), \\
& A=\left(\begin{array}{ll}
a & b \\
c & d
\end{array}\right), \\
& A^{-1}=\left(\begin{array}{cc}
d & -b \\
-c & a
\end{array}\right) / \operatorname{det} A
\end{aligned}
$$

From $(22.8])$ we obtain

$$
q_{n+1}=1+1 / q_{n-1}, q_{n \pm 1}=z_{n \pm 1} / z_{n}, z_{n \pm 1}=q_{n \pm 1} z_{n}
$$

When this iteration process converges: $q_{n} \rightarrow q$,

$$
q=1+1 / q \Rightarrow q_{+}=\frac{\sqrt{5}+1}{2}, q_{-}=\frac{-\sqrt{5}+1}{2}
$$

\subsection{Bessel function (BF)}

BF are the canonical solutions $y(z)$ of Bessel's differential equation

$$
\left(z^{2} \partial_{z}^{2}+z \partial_{z}+\left(z^{2}-\alpha^{2}\right) y(z)=0\right.
$$

for an arbitrary complex number $\alpha$ (the order of the Bessel function). The most important cases are for $\alpha$ an integer or half-integer. Although $\alpha$ and $-\alpha$ produce the same differential equation for real $\alpha$, it is conventional to define different Bessel functions for these two values in such a way that the Bessel functions are mostly smooth functions of $\alpha$. Bessel's equation arises when finding separable solutions to Laplace's equation and the Helmholtz equation in cylindrical or spherical coordinates. Bessel functions are therefore especially important for many problems of wave propagation and static potentials. In solving problems in cylindrical coordinate systems, one obtains Bessel functions of integer order $(\alpha=n)$; in spherical problems, one obtains half-integer orders $(\alpha$ $=\mathrm{n}+1 / 2)$. Bessel functions of the first kind, denoted as $J_{\alpha}(z)$, is possible to define the function by its Taylor series expansion around $\mathrm{z}=0$ :

$$
\begin{aligned}
& J_{\alpha}(z)=\sum_{n \geq 0}(-1)^{n}(n ! \Gamma(n+1+\alpha))^{-1}\left(\frac{z}{2}\right)^{2 n+\alpha} \\
& =(z / 2)^{\alpha}\left(\Gamma\left(\delta_{z} / 2+1\right) \Gamma\left(\delta_{z} / 2+1+\alpha\right)\right)^{-1}\left(1+(z / 2)^{2}\right)^{-1}
\end{aligned}
$$

where $\Gamma(z)$ is the gamma function, a generalization of the factorial function to non-integer values. The Bessel function of the first kind is an entire function if $\alpha$ is an integer. For non-integer $\alpha$, the functions $J_{\alpha}(x)$ and $J_{-\alpha}(x)$ are linearly independent, and are therefore the two solutions of the differential equation. For integer $\alpha, J_{-n}(x)=(-1)^{n} J_{n}$, and the second linearly independent solution is then found to be the Bessel function of the second kind.

\subsection{Bessel's integrals}

Bessel's integrals

$$
J_{n}(z)=\int_{-\pi}^{\pi} \frac{d t}{2 \pi} e^{i(n t-z \sin t)}=\int_{0}^{\pi} \frac{d t}{\pi} \cos (n t-z \sin t)
$$


The definition may be extended to non-integer orders by (for $\operatorname{Re}(\mathrm{x})>0$ )

$$
J_{-\alpha}(z)=\int_{0}^{\pi} \frac{d t}{\pi} \cos (\alpha t-z \sin t)+\frac{\sin \alpha \pi}{\pi} \int_{0}^{\pi} d t e^{-\alpha t-z \sinh (t)}
$$

\subsection{Relation to hypergeometric functions and Laguerre polynomials}

The Bessel functions can be expressed in terms of the generalized hypergeometric series as:

$$
\begin{aligned}
& J_{\alpha}(z)=\frac{\left(\frac{x}{2}\right)^{\alpha}}{\Gamma(\alpha+1)}{ }_{0} F_{1}\left(\alpha+1,-\frac{z^{2}}{2}\right) \\
& =\frac{\left(\frac{x}{2}\right)^{\alpha} e^{-t}}{\Gamma(\alpha+1)} \sum \frac{t^{n}}{n !} \frac{L_{n}^{\alpha}\left(\frac{z^{2}}{4 t}\right)}{\left(\begin{array}{c}
n+\alpha \\
n
\end{array}\right)}
\end{aligned}
$$

\subsection{Spherical Bessel functions}

When solving the Helmholtz equation in spherical coordinates by separation of variables, the radial equation has the form:

$$
\left(z^{2} \partial_{z}^{2}+2 z \partial_{z}+\left(z^{2}-n(n+1)\right)\right) y=0
$$

The two linearly independent solutions to this equation are called the spherical Bessel functions $j_{n}$ and $y_{n}$, and are related to the ordinary Bessel functions $J_{n}$ and $Y_{n}$ by:

$$
\begin{aligned}
j_{n}(z) & =\sqrt{\frac{\pi}{2 z}} J_{n+\frac{1}{2}}(z)=(-1)^{n} z^{n}\left(\frac{d}{z d z}\right)^{n} \frac{\sin z}{z} \\
y_{n}(z) & =\sqrt{\frac{\pi}{2 z}} Y_{n+\frac{1}{2}}(z)=(-1)^{n+1} \sqrt{\frac{\pi}{2 z}} J_{-n-\frac{1}{2}}(z)=(-1)^{n+1} z^{n}\left(\frac{d}{z d z}\right)^{n} \frac{\cos z}{z}
\end{aligned}
$$

RiccatiÜBessel functions $S_{n}, C_{n}$ only slightly differ from spherical Bessel functions:

$$
\begin{aligned}
& S_{n}(z)=x j_{n}(z) \sqrt{\frac{\pi z}{2}} J_{n+\frac{1}{2}}(z) \\
& C_{n}(z)=-z y_{n}(z)=-\sqrt{\frac{\pi z}{2}} Y_{n+\frac{1}{2}}(z)
\end{aligned}
$$

They satisfy the differential equation:

$$
\left(z^{2} \partial_{z}^{2}+\left(z^{2}-n(n+1)\right)\right) y=0
$$

Another way to define the Bessel functions is the Poisson representation formula and the Mehler-Sonine formula:

$$
\begin{aligned}
& J_{\alpha}(z)=a_{\alpha} z^{\alpha} \int_{-1}^{1} d s e^{i z s}\left(1-s^{2}\right)^{\alpha-\frac{1}{2}}=b_{\alpha} z^{-\alpha} \int_{1}^{\infty} \frac{d u \sin (u z)}{\left(u^{2}-1\right)^{\alpha+\frac{1}{2}}} \\
& a_{\alpha}=\left(\sqrt{\pi} 2^{\alpha} \Gamma\left(\alpha+\frac{1}{2}\right)\right)^{-1}, b_{\alpha}=2^{\alpha+1}\left(\sqrt{\pi} \Gamma\left(\frac{1}{2}-\alpha\right)\right)^{-1}, \alpha>-\frac{1}{2}, z \in \mathbb{C}
\end{aligned}
$$




\subsection{Recurrence relations and corresponding time-reversible dynamical system}

The functions $J_{\alpha}(z)$ and $Y_{\alpha}(z)$ satisfy the recurrence relations:

$$
\begin{aligned}
& Z_{\alpha+1}(z)=\frac{2 \alpha}{z} Z_{\alpha}(z)-Z_{\alpha-1}(z), \\
& Z_{\alpha+1}(z)=-2 \partial_{z} Z_{\alpha}(z)+Z_{\alpha-1}(z), Z=J, Y
\end{aligned}
$$

from which we obtain

$$
\begin{aligned}
& \Phi_{\alpha+1}=A \Phi_{\alpha}, \\
& \Phi_{\alpha+1}=\left(\begin{array}{c}
Z_{\alpha+1} \\
Z_{\alpha+2}
\end{array}\right), \Phi_{\alpha}=\left(\begin{array}{c}
Z_{\alpha-1} \\
Z_{\alpha}
\end{array}\right), \\
& A=\left(\begin{array}{cc}
-1 & 2 \alpha / z \\
-2(\alpha+1) / z & 2 \alpha 2(\alpha+1) / z^{2}-1
\end{array}\right), \operatorname{det} A=1, A^{-1}=\left(\begin{array}{cc}
2 \alpha 2(\alpha+1) / z^{2}-1-2 \alpha / z \\
2(\alpha+1) / z & -1
\end{array}\right), \\
& A=\left(\begin{array}{cc}
a & b \\
c & d
\end{array}\right), \\
& A^{-1}=\left(\begin{array}{cc}
d & -b \\
-c & a
\end{array}\right) / \operatorname{det} A
\end{aligned}
$$

One can compute Bessel functions of higher orders (or higher derivatives) given the values at lower orders (or lower derivatives). In particular,

$$
\begin{aligned}
& \eta_{z}^{m}\left(z^{\alpha} Z_{\alpha}(z)\right)=z^{\alpha-m} Z_{\alpha-m}(z), \\
& \eta_{z}^{m}\left(Z^{-\alpha} Z_{\alpha}(z)\right)=(-1)^{m} z^{-\alpha-m} Z_{\alpha+m}(z), \eta_{z}=\frac{d}{z d z}
\end{aligned}
$$

Problem: consider proper generalization for non integer values of $m$.

Modified Bessel functions follow similar relations:

$$
\exp \left(\left(t+t^{-1}\right) x / 2\right)=\sum_{n=-\infty}^{\infty} I_{n}(x) t^{n}
$$

\subsection{Time-reversible dynamical system for Bessel functions}

The recurrence relation for Modified Bessel functions reads

$$
\begin{aligned}
& C_{\alpha+1}(z)=-\frac{2 \alpha}{z} C_{\alpha}+C_{\alpha-1}(z), \\
& C_{\alpha+1}(z)=2 \partial_{z} C_{\alpha}-C_{\alpha-1}(z), C_{\alpha}=I_{\alpha}, \exp (i \pi \alpha) K_{\alpha}
\end{aligned}
$$

from which we obtain

$$
\begin{aligned}
& \Phi_{\alpha+1}=A \Phi_{\alpha}, \\
& \Phi_{\alpha+1}=\left(\begin{array}{c}
C_{\alpha+1} \\
C_{\alpha+2}
\end{array}\right), \Phi_{\alpha}=\left(\begin{array}{c}
C_{\alpha-1} \\
C_{\alpha}
\end{array}\right), \\
& A=\left(\begin{array}{cc}
1 & -2 \alpha / z \\
-2(\alpha+1) / z & 1+2 \alpha 2(\alpha+1) / z^{2}
\end{array}\right), \operatorname{det} A=1, A^{-1}=\left(\begin{array}{cc}
2 \alpha 2(\alpha+1) / z^{2} & 2 \alpha / z \\
2(\alpha+1) / z & 1
\end{array}\right),
\end{aligned}
$$




$$
\begin{aligned}
& A=\left(\begin{array}{ll}
a & b \\
c & d
\end{array}\right), \\
& A^{-1}=\left(\begin{array}{cc}
d & -b \\
-c & a
\end{array}\right) / \operatorname{det} A
\end{aligned}
$$

\section{A Way to the Solution of the Traveling Salesman Problem (TSP) with Quanputing}

The $N P \stackrel{?}{=} P$ problem will be solved if for some $N P$ - complete problem, e.g. TSP, a polynomial algorithm find; or show that there is not such an algorithm; or show that it is impossible to find definite answer to that question.

TSP means to find minimal length path between $N$ fixed points on a surface, which attends any point ones. We consider a system where $N$ points with quenched positions $x_{1}, x_{2}, \ldots, x_{N}$ are independently distributed on a finite domain $D$ with a probability density function $p(x)$. In general, the domain $D$ is multidimensional and the points $x_{n}$ are vectors in the corresponding Euclidean space. Inside the domain $D$ we consider a polymer chain composed of $N$ monomers whose positions are denoted by $y_{1}, y_{2}, \ldots, y_{N}$. Each monomer $y_{n}$ is attached to one of the quenched sites $x_{m}$ and only one monomer can be attached to each site. The state of the polymer is described by a permutation $\sigma \in \Sigma_{N}$ where $\Sigma_{N}$ is the group of permutations of $N$ objecs.

The Hamiltonian for the system is given by

$$
H=\sum_{n=1}^{N} V\left(\left|y_{n}-y_{n-1}\right|\right)
$$

Here $V$ is the interaction between neighboring monomers on the polymer chain. For convenience the chain is taken to be closed, thus we take the periodic boundary condition $x_{0}=x_{N}$. A physical realization of this system is one where the $x_{n}$ are impurities where the monomers of a polymer loop are pinned. In combinatorial optimization, if one takes $V(x)$ to be the norm, or distance, of the vector $x$ then $H(\sigma)$ is the total distance covered by a path which visits each site $x_{n}$ exactly once. The problem of finding $\sigma_{0}$ which minimizes $H(\sigma)$ is known as the traveling salesman problem (TSP) Gutin, Pannen, 2002].

In field theory language to the TSP we correspond the calculation of the following correlator

$$
\begin{aligned}
& G_{2 N}\left(x_{1}, x_{2}, \ldots, x_{N}\right)=Z_{0}^{-1} \int d \varphi(x) \varphi^{2}\left(x_{1}\right) \varphi^{2}\left(x_{2}\right) \ldots \varphi^{2}\left(x_{N}\right) e^{-S(\varphi)} \\
& =\frac{\delta^{2 N} F(J)}{\delta J\left(x_{1}\right)^{2} \ldots \delta J\left(x_{N}\right)^{2}}, F(J)=\ln Z(J), \\
& Z(J)=\int d \varphi e^{-\frac{1}{2} \varphi \cdot A \cdot \varphi+J \cdot \varphi}=e^{\frac{1}{2} J \cdot A^{-1} \cdot J}, A^{-1}(x, y ; m)=e^{-m|x-y|}, \\
& L_{\text {min }}\left(x_{1}, \ldots, x_{N}\right)=-\frac{d}{d m} \ln G_{2 N s}+O\left(e^{-a m}\right) \\
& <A^{-1}>\equiv \frac{1}{\Gamma(s)} \int_{0}^{\infty} d m m^{s-1} A^{-1}(x, y ; m)=\frac{1}{|x-y|^{s}} \\
& =L_{s} A^{-1}(x-y ; s) \\
& k(d) \Delta_{d} L_{s} A^{-1}(x ; s)=\delta^{d}(x) \Rightarrow A(x ; s)=k(d) \Delta_{d} L_{s}, \\
& s=d-2 ; \varphi=\varphi(x, m) .
\end{aligned}
$$


If we take relativistic massive scalar field, then $A=\Delta_{d}+m^{2}$,

$$
A^{-1}(x) \sim|x|^{2-d} e^{-m|x|},
$$

and for $d=2$, we also have the needed behaviour. Note that $G_{2 N}$ is symmetric with respect to its arguments and contains any paths including minimal length one.

\section{The Theory Space (TS) and Fundamental Constants}

Theoretical equations describing the physical world deal with dimensionless quantities and their solutions depend on dimensionless fundamental parameters, like $\alpha^{-1} \simeq 137$. But experiments, from which these theories are extracted and by which they could be tested, involve measurements, i.e. comparisons with standard dimensionful scales. Without standard dimensionful units and hence without certain conventions physics is unthinkable.

According to the high school physics, there are three basic quantities in Nature: Length, Mass and Time. All other quantities, such as electric charge or temperature, occupied a lesser status since they could all be re-expressed in terms of these basic three. As a result, there are three basic units: centimeter $(\mathrm{cm})$, gram $(\mathrm{g})$ and second $(\mathrm{s})$, reflected in the three-letter name "CGS" system (or perhaps meter, kilogram and second in the alternative, but still three-letter, "MKS" system).

In quantum mechanics, there is a minimum quantum of action given by PlanckŠs constant $\hbar$; in special relativity there is a maximum velocity given by the velocity of light $c$; in classical gravity the strength of the force between two objects is determined by Newton's constant of gravitation $G$. In terms of length, time and mass their dimensions are

$$
\begin{aligned}
& {[c]=L T^{-1},} \\
& {[\hbar]=L^{2} T^{-1} M} \\
& {[G]=L^{3} T^{-2} M^{-1}}
\end{aligned}
$$

Max Planck identified a century ago three basic units, the Planck length $l_{p}$, the Planck time $t_{p}$ and Planck mass $m_{p}$ :

$$
\begin{aligned}
& l_{p}=\sqrt{\frac{G \hbar}{c^{3}}}=1.616 \times 10^{-35} \mathrm{~m} \\
& t_{p}=\sqrt{\frac{G \hbar}{c^{5}}}=5.390 \times 10^{-44} \mathrm{~s} \\
& m_{p}=\sqrt{\frac{c \hbar}{G}}=2.177 \times 10^{-8} \mathrm{~kg}
\end{aligned}
$$

Note that, unlike $\hbar$ and $c$, the dimension of $G$ depends on dimension of space-time D:

$$
\begin{aligned}
& F=G \frac{m M}{r^{D-2}}=m a, \Downarrow \\
& {\left[G_{D}\right]=L^{D-1} T^{-2} M^{-1}}
\end{aligned}
$$


so,

$$
\begin{aligned}
& \hbar G_{D}=l_{p D}^{D+1} t_{p D}^{-3}, \\
& c=l_{p D} t_{p D}^{-1}, \Downarrow \\
& l_{p D}^{D-2}=\frac{\hbar G_{D}}{c^{3}}, \\
& t_{p D}^{D-2}=\frac{\hbar G_{D}}{c^{D+1}}, \\
& m_{p D}^{D-2}=\frac{c^{5-D} \hbar^{D-3}}{G_{D}}
\end{aligned}
$$

After compactification to four dimensions,

$$
G_{D}=v G_{4}
$$

where $v$ - the volume of the compactifying manifold has the four-dimensional interpretation as the vacuum expectation value of scalar modulus fields coming from the internal components of the metric tensor, it depends on the choice of vacuum but does not introduce any more fundamental constants into the lagrangian.

Note that in the gravity coupling constant and corresponding unites (24.4), the dimention $D$ can takes also non integer-fractal values.

In the 1870's G.J. Stoney [Stoney, 1881], the physicist who coined the term "electron" and measured the value of elementary charge $e$, introduced as universal units of Nature for $L, T, M$ :

$$
\begin{aligned}
& l_{S}=\frac{e}{c^{2}} \sqrt{G} \\
& t_{S}=\frac{e}{c^{3}} \sqrt{G} \\
& =\frac{l_{S}}{c}, \\
& m_{S}=\frac{e}{\sqrt{G}} \\
& l_{S} m_{S}=\frac{e^{2}}{c^{2}}
\end{aligned}
$$

The expression for $m_{S}$ has been derived by equating the Coulomb and Newton forces,

$$
e^{2}=G m^{2} \Rightarrow m_{S}=\frac{e}{\sqrt{G}}
$$

The expressions for $l_{S}$ and $t_{S}$ has been derived from $m_{S}, c$ and $e$ on dimensional grounds,

$$
\left[\frac{e^{2}}{r^{2}}\right]=[m a]=M L T^{-2} \Rightarrow e^{2}=m_{S} L^{3} T^{-2}=m_{S} l_{S} c^{2} \Rightarrow l_{S}=\frac{e^{2}}{c^{2} m_{S}}=\frac{e \sqrt{G}}{c^{2}}
$$

Note that, we can define the units of Nature from fundamental length- $l$, charge- $e$ and speed of light- $c$

$$
t=l / c, m=\left(\frac{e}{c}\right)^{2} / l, G=\left(\frac{l c^{2}}{e}\right)^{2}
$$


When M. Planck discovered in $1899 h$ he introduced [Planck, 1899] as universal units of Nature for L, T, M:

$$
\begin{aligned}
& m_{P}=\sqrt{\frac{h c}{G}}=\frac{m_{S}}{\sqrt{\alpha}}, \\
& l_{P}=\frac{h}{c m_{P}}=\frac{l_{S}}{\sqrt{\alpha}}=11.7 l_{S}, \\
& t_{P}=\frac{l_{P}}{c}=\frac{t_{S}}{\sqrt{\alpha}}
\end{aligned}
$$

Max Planck invented the system of fundamental unites $c, h, G$ and $k$. G. Gamov, D. Ivanenko and L. Landau [Gamov, Ivanenko, Landau, 1928] considered the system without the parameter $k$, as fundamental one. Bronshtein [Bronshtein, 1933] and Zelmanov [Zelmanov, 1967], developed the idea of the cube of theories. The cube is located along three orthogonal axes marked by $c$ (actually by $1 / c), \hbar, G$. The vertex (000) corresponds to nonrelativistic mechanics, (c00) - to special relativity, $(0 \hbar 0)$ - to non-relativistic quantum mechanics, $(c \hbar 0)$ - to quantum field theory, $(c 0 G)$ - to general relativity, $(c \hbar \mathrm{G})$ - to futuristic quantum gravity and the Theory of Everything, TOE, modern version of which is M-theory. There is a hope that in the framework of TOE the values of dimensionless fundamental parameters will be ultimately calculated. Note that 3-dimensional TS-chG where invented for 3-dimensional space models, d-dimensional theory may need d-dimensional TS, but, as we have seen, when extra dimensions are compactified the TS remain 3-dimensional; Stoney's fundamental constants are more fundamental just because they are less than Planck's constants :)

\subsection{The Practical Meaning of Units}

The meter was defined in 1791 as a 1/40 000000 part of Paris meridian.The gram is the mass of one cubic $\mathrm{cm}$ of water. The $\mathrm{cm}$ and $\mathrm{sec}$ are connected with the size and rotation of the earth. An important step forward was made in the middle of XX century, when the standards of $\mathrm{cm}$ and sec were defined in terms of of wave-length and frequency of a certain atomic line.

Enormously more universal and fundamental are $c$ and $\hbar$ given to us by Nature herself as units of velocity $[v]=[L / T]$ and angular momentum $[J]=[M v L]=\left[M L^{2} / T\right]$ or action $[S]=[E T]=$ $\left[M v^{2} T\right]=\left[M L^{2} / T\right]$.

\subsection{The Fundamental Meaning of $c$}

It is important that $c$ is not only the speed of light in vacuum. What is much more significant is the fact that it is the maximal velocity of any object in Nature, the photon being only one of such objects. The fundamental character of $c$ would not be diminished in a world without photons. The fact that $c$ is the maximal $v$ leads to new phenomena, unknown in newtonian physics and described by relativity. Therefore Nature herself suggests $c$ as fundamental unit of velocity.

$c$ is more fundamental than $\alpha$ because it is the basis of relativity theory which unifies space and time, as well as energy, momentum and mass. 


\subsection{The Fundamental Meaning of $\hbar$}

The quantity $\hbar$ is also fundamental: it is the quantum of the angular momentum $J$ and a natural unit of the action $S$. When $J$ or $S$ are close to $\hbar$, the whole realm of quantum mechanical phenomena appears. Particles with integer $J$ (bosons) tend to be in the same state (i.e. photons in a laser, or Rubidium atoms in a drop of Bose-Einstein condensate). Particles with half-integer $J$ (fermions) obey the Pauli exclusion principle which is so basic for the structure of atoms, atomic nuclei and neutron stars. Symmetry between fermions and bosons, dubbed supersymmetry or SUSY, is badly broken at low energies, but many theorists believe that it is restored near the Planck mass in particular in superstrings and M-theories. It is natural when dealing with quantum mechanical problems to use $\hbar$ as the unit of $J$ and $S$.

\subsection{A Fundamental Meaning of $G$}

The status of $G$ and its derivatives, $m, l, t$, is at present different from that of $c$ and $\hbar$, because the quantum theory of gravity is still under construction. The majority of experts connect their hopes with extra spatial dimensions and superstrings. The characteristic length of a superstring $l_{s}\left(M_{G U T}^{2}\right)=l_{P} / \sqrt{\alpha\left(M_{G U T}^{2}\right)}$. Possible modifications of NewtonŠs potential at sub-millimetre distances demonstrates that the position of $G$ is not as firm as that of $c$ and $\hbar$. If the theory of gravity reduce to more fundamental structures, like old theory of weak interections with its coupling constant $G$ reduce to SM, than gravitation coupling constant become calculable in terms of the fundamental theory. The Newtonian potential around the sun is for non-vanishing $\Lambda$ modified to [Axenides, Floratos, Perivolaropoulos, 2000], [Gibbons, Hawking, 1977]

$$
V(r)=\frac{G M}{r}+\frac{\Lambda c^{2}}{6} r^{2}
$$

where $M$ is the mass of the sun and $r$ the distance from the sun.

\subsection{Temperature and Meaning of Boltzmann's Constant $k$}

Mathematically temperature $T$ is defined as a derivative of internal energy $E$ of a system over its entropy $S$ :

$$
\begin{aligned}
& Z(\beta)=\sum_{n} e^{-\beta E_{n}}=\sum_{E_{n}} N\left(E_{n}\right) e^{-\beta E_{n}}=\sum_{E_{n}} e^{-\beta F_{n}}=e^{-\beta F} \\
& F=E-T S=E-t S_{B}, T=\beta^{-1}=k t, S_{B}=k S \\
& \left(\frac{\partial F}{\partial S}\right)_{T}=0 \Rightarrow T=\frac{d E}{d S}, k=8.69 \times 10^{-5} \mathrm{eV} / K=1.38 \times 10^{-23} \mathrm{~J} / \mathrm{K} .
\end{aligned}
$$

As temperature is an average energy of an ensemble of particles, it is natural to measure it in units of energy. So, the Boltzmann's constant $k$ connects microscopic phenomena to macroscopic one but it is not necessary to have different unit for measuring temperature and corresponding dimensional coefficient $k, T=k t$. We can put $k=1$ and measuring the temperature in energy unites. In this sense, the Boltzmann's Constant $k$ has not the fundamental meaning. 


\subsection{Fundamental Constants}

There are different opinions about the number of fundamental constants [Duff, Okun, Veneziano, 2001]. According to Okun there are three fundamental dimensionful constants in Nature: Planck's constant, $\hbar$; the velocity of light, $c$; and Newton's constant, $G$. According to Veneziano, there are only two: the string length $l_{s}$ and $c$. According to Duff, there are not fundamental constants at all.

\subsection{High-energy theories and Fundamental Constants}

5-dimensional Einstein-Hilbert action

$$
S=\left(12 \pi^{2} G_{5}\right)^{-1} \int d^{5} x \sqrt{-g_{5}} R_{5}
$$

Decomposing 5-dimensional metric as

$$
g_{5}=\left(\begin{array}{cc}
g_{\mu v}+\phi^{2} A_{\mu} A_{v} / M^{2} & \phi A_{\mu} / M \\
\phi A_{v} / M & \phi^{2}
\end{array}\right),
$$

we obtain

$$
S=\left(16 \pi G_{4}\right)^{-1} \int d^{4} x \sqrt{-g_{4}} \phi\left(R_{4}-\frac{\phi^{2}}{4 M^{2}} F^{2}\right)
$$

where the 4-dimensional gravitational constant $G_{4}$ is

$$
G_{4}=G_{5} \frac{3 \pi}{4} / \int d x_{5}
$$

The scalar field couples explicitly to the kinetic term of the vector field and cannot be eliminated by a redefinition of the metric. Such dependencies of the masses and couplings are generic for higherdimensional theories and in particular string theory. It is actually one of the definitive predictions for string theory that there exists a dilaton, that couples directly to matter [Taylor, Veneziano, 1988] and whose vacuum expectation value determines the string coupling constants [Witten, 1984,2].

\subsection{Fundamental Constants from String Theory}

In the Nambu-Goto string model

$$
\frac{S}{\hbar}=\frac{1}{s} \int d(\text { Area }), s=l_{s}^{2}
$$

where $l_{s}$ is the characteristic size of strings. The characteristic length of a superstring

$$
l_{s}\left(M_{G U T}^{2}\right)=\frac{l_{P}}{\sqrt{\alpha\left(M_{G U T}^{2}\right)}}
$$

We have seen, that $\alpha_{G U T}^{-1}$ in MSSM is equal to 29, so, in String Minimal SM (SMSM)

$$
s=\frac{l_{p}^{2}}{\alpha_{G U T}}=\frac{l_{s}^{2}}{\alpha_{G U T} \alpha\left(m_{e}\right)}=29 \times 137 e^{2} G / c^{4}
$$


where $l_{s}$ is Stony's fundamental length,

$$
l_{s}=\frac{l_{p}}{\sqrt{\alpha\left(m_{e}\right)}}, \alpha\left(m_{e}\right)^{-1}=137 .
$$

the parameter $s$ is the one which replace the gravitational constant in old triumvirate of fundamental units $G, c, \hbar \Rightarrow s, c, \hbar$. Important consequence of this statement is that a string theory phenomenon we observe in everyday live as gravitation force.

String theory only needs two fundamental dimensionful constants $c$ and s, i.e. one fundamental unit of speed and one of area. The role of Planck constant plays $s$.

There is, in relativity, a fundamental unit of speed $c$; there is, in $\mathrm{QM}$, a fundamental unit of action $\hbar$; there is, in string theory, a fundamental unit of action - area, $s$.

In string theory we would like to freeze the moduli at values that provide the correct values of the coupling constant and unification scale of grand unified theories (GUTs). For instance, the dilaton and compactification volume $V_{6}$ should be frozen at values such that

$$
\alpha_{G U T} \sim e^{\phi} \sim \frac{m_{s}^{2}}{m_{P}^{2}} \sim g_{s}, m_{G U T}^{2} \sim \alpha_{G U T}^{4 / 3} g_{s}^{-1 / 3} m_{P}^{2}, g_{s}=V_{6} M^{6} e^{\phi}
$$

where $m_{G U T}, m_{s}, m_{P}$ are GUT, string and the Planck scales, $g_{s}$ is the string coupling.

\subsection{Effective Action of String Theory}

The tree-level low-energy effective action of string theory reads:

$$
S=\frac{1}{2} \int d^{4} x \sqrt{-g} e^{-\phi}\left(\lambda_{s}^{-2}\left(R+\partial_{\mu} \phi \partial^{\mu} \phi+H_{\mu v \rho} H^{\mu v \rho}\right)+F_{\mu v} F^{\mu v}\right)
$$

where $H_{\mu \nu \rho}$ is Kalb-Ramond antisymmetric tensor field strength. Couplings are VEVs which, hopefully, become dynamically determined. In particular, a scalar field, the so-called dilaton $\phi$, controls all sorts of couplings, gravitational and gauge alike,

$$
\alpha_{\text {gauge }} \sim e^{\phi} \sim \frac{l_{p}^{2}}{l_{s}^{2}}=G_{N} T, T=\frac{\hbar}{l_{s}^{2}}
$$

where $l_{s}$ is string length, $T$ is string tension.

\subsection{Mathematical Foundation for Fundamental Constants}

In mathematics we have two kind of structures, discrete and continuous one. If a physical quantity has discrete values, it might not have dimension. If the values are continuous - the quantity might have dimension, unit of measure. These structures may depend on scale, e.g. on macroscopic scale condensed state of matter (and time) is well described as continuous medium, so we use dimensional units of length (and time). On the scale of atoms, the matter has discrete structure, so we may count lattice sites and may not use unit of length. If at small (e.g. at Plank) scale space (and/or time) is discrete then we not need an unit of length (time) for measuring, there is the fundamental length and we can just count. 


\section{The Growth Points (GPs)}

\subsection{Toward M-Theory}

Today a mathematical structure that underlies and explains all of the physical world consists of two distinct parts: Relativistic Quantum Field Theory and General Relativity. General Relativity is classical, and has not been successfully quantized (yet) whereas the known consistent quantum field theories do not include gravity.

The fundamental theory might be:

Mathematically consistent;

Experimentally correct;

Predictive;

Unique;

We now think that the various string theories are actually five different perturbative expansions of a single theory known as M-Theory. So this is our current best guess for a fundamental theory of everything.

\subsection{U-geometry}

While Riemannian geometry singles out the spacetime metric, $g_{m n}$, as its only fundamental geometric object, T-duality in string theory or U-duality in M-theory put other form-fields at an equal footing along with the metric. As a consequence, Riemannian geometry appears incapable of manifesting the duality, especially in the formulations of low energy effective actions. Novel differential geometry beyond Riemann is desirable which treats the metric and the form-fields equally as geometric objects, and makes the covariance apparent under not only diffeomorphism but also duality transformations. Despite of recent progress in various limits, eleven-dimensional $M$-theory remains still $M$ ysterious, not to mention its full U-duality group which was conjectured to correspond to a certain Kac-Moody algebra, or an exceptional generalized geometry called $E 11$

\subsection{M-theory and Quanputing}

Whenever two very different areas of theoretical physics are found to share the same mathematics, it frequently leads to new insights on both sides. Knowledge of string theory and M-theory leads to new discoveries about Quantum Information Theory (QIT) and vice-versa [Duff, 2007].

\subsection{Normal Quantum Hall Effect (NQHE)}

The quantization of the Hall conductance has the important property of being incredibly precise. This phenomenon, referred to as "exact quantization", has been shown to be a subtle manifestation of the principle of gauge invariance [Laughlin, 1981]. It has allowed for the definition of a new practical standard for electrical resistance, based on the resistance quantum given by the von Klitzing constant

$$
R_{K}=h / e^{2}=25812.807557(18) \Omega
$$

This is named after Klaus von Klitzing, the discoverer of exact quantization. Since 1990, a fixed conventional value $R_{K-90}$ is used in resistance calibrations worldwide [एzalenchuk, 2010]. 
The NQHE, or integer quantum Hall effect, is a quantum-mechanical version of the Hall effect, observed in two-dimensional electron systems subjected to low temperatures and strong magnetic fields, in which the Hall conductivity $\sigma$ takes on the quantized values

$$
\sigma=\frac{I}{V}=\frac{e^{2}}{h} q=2 q \frac{\alpha}{\hbar}, \hbar=\frac{h}{2 \pi}, \alpha=\frac{e^{2}}{4 \pi}
$$

were $I$ is the channel current, $V$ is the Hall voltage, $e$ is the elementary charge and $h$ is Planck's constant. The $q$ is known as the "filling factor", and can take on either integer, $q=n=1,2,3, \ldots$ or fractional values.

\subsection{Anomalous Quantum Hall Effect (AQHE)}

For the AQHE, or fractional quantum Hall effect, the factor $q$ takes fractional values, $n=1 / 3$, $2 / 3,1 / 5,2 / 5,3 / 5, \ldots$

So, the quantum Hall effect is referred to as the normal (integer) or fractional (anomalous) quantum Hall effect depending on whether $q$ is an integer or fraction respectively. The integer quantum Hall effect is very well understood, and can be simply explained in terms of single-particle orbitals of an electron in a magnetic field. The fractional quantum Hall effect is more complicated, as its existence relies fundamentally on electron-electron interactions. Although the microscopic origins of the fractional quantum Hall effect are unknown, there are several phenomenological approaches that provide accurate approximations. For example the effect can be thought of as an integer quantum Hall effect, not of electrons but of charge-flux composites known as composite fermions. There is also a new concept of the quantum spin Hall effect which is an analogue of the quantum Hall effect, where spin currents flow instead of charge currents.

\subsection{Mesons under magnetic fields}

Strong magnetic fields with strength of $10^{18} \sim 10^{20} G$ (corresponding to $e B(0.1-1.0 \mathrm{GeV})^{2}$ ), can be generated in the laboratory through non-central heavy ion collisions at the Relativistic Heavy Ion Collider (RHIC) and the Large Hadron Collider (LHC). In the surface of magnetars, magnetic fields can reach $10^{14-15} \mathrm{G}$, which is thousand of times larger than that of an average pulsar, and in the inner core of magnetars the magnetic fields could reach as high as $10^{18} \sim 10^{20} \mathrm{G}$. Therefore, it is important to understand the properties of Quantum Chromodynamics (QCD) vacuum and hot/dense quark matter under strong magnetic fields. Progresses have been made in this field includ the Chiral Magnetic Effect (CME), Chiral Vortical Effect (CVE), the Magnetic Catalysis and Inverse Magnetic Catalysis, and the Vacuum Superconductor.

\subsection{The Theory Space (TS)}

Max Planck during years 1897-1899 invented the system of fundamental unites $c, h, G$ and $k$ G. Gamov, D. Ivanenko and L. Landau [Gamov, Ivanenko, Landau, 1928] considered the system without the parameter $k$, as fundamental one. Bronshtein [Bronshtein, 1933] and Zelmanov [Zelmanov, 1967], developed the idea of the cube of theories. The cube is located along three orthogonal axes marked by $c$ (actually by $1 / c$ ), $\hbar, G$. The vertex (000) corresponds to nonrelativistic mechanics, (c00) - to special relativity, $(0 \hbar 0)$ - to non-relativistic quantum mechanics, $(c \hbar 0)$ to quantum field theory, $(c 0 G)$ - to general relativity, $(c \hbar \mathrm{G})$ - to futuristic quantum gravity and 
the Theory of Everything, TOE, modern version of which is M-theory. There is a hope that in the framework of TOE the values of dimensionless fundamental parameters will be ultimately calculated. Note that 3-dimensional TS-chG where invented for 3-dimensional space models, ddimensional theory may need d-dimensional TS.

\subsection{Fractal dynamical systems}

The differential operator in superspace, i.e., the superderivative, and its inverse, are

$$
D=\frac{\partial}{\partial \theta}+\theta \frac{\partial}{\partial t}=\partial_{\theta}+\theta \partial_{t}, D^{-1}=\theta+\partial_{\theta} \partial_{t}^{-1}
$$

were, $\theta$ is a Grassmann odd variable, accordingly the square of $D$ turns out to be ordinary derivative,

$$
D^{2}=\partial_{t}
$$

in other words $D$ can be regarded as a square root of $\partial_{t}$.

\subsection{Inverse Problem of Fructal Calculus (IPFC) with Applications}

The final form of the laws obeyed by the electromagnetic field was found by Maxwell, around 1860 - these laws survived relativity and quantum theory, unharmed. Gauge invariance allows only two free parameters in the Lagrangian of this system: $e, m_{e}$. Moreover, only one of these is dimensionless:

$$
\alpha=\frac{e^{2}}{4 \pi}=1 / 137.035999074(44)
$$

$\mathrm{U}(1)$ symmetry and renormalizability fully determine the properties of the e.m. interaction, except for this number, which so far still remains unexplained.

In 1965, Vanyashin and Terentyev [Vanyashin, Terentyev, 1965] found that the renormalization of the electric charge of a vector field is of opposite sign to the one of the electron. In the language of $\mathrm{SU}(2)$ gauge field theory, their result implies that the $\beta$-function is negative at one loop. The first correct calculation of the $\beta$-function of a nonabelian gauge field theory was carried out by Khriplovich, for the case of SU(2), relevant for the electroweak interaction [Khriplovich, 1969]. He found that $\beta$ is negative and concluded that the interaction becomes weak at short distance. In his $\mathrm{PhD}$ thesis, 't Hooft performed the calculation of the $\beta$-function for an arbitrary gauge group, including the interaction with fermions and Higgs scalars [t Hooft, 1971]. He demonstrated that the theory is renormalizable and confirmed that, unless there are too many fermions or scalars, the $\beta$-function is negative at small coupling. In 1973, Gross and Wilczek [Gross, Wilczek, 1973] and Politzer [Politzer, 1973] discussed the consequences of a negative $\beta$-function and suggested that this might explain Bjorken scaling, which had been observed at SLAC in 1969. They pointed out that QCD predicts specific modifications of the scaling laws. In the meantime, there is strong experimental evidence for these. 


\subsection{Renormdynamics of the spacetime}

Geometry of the spacetime can change with scale. Different approaches to quantum gravity, ranging from asymptotic safety, non-commutative geometry, causal dynamical triangulations, and spin-foams to fractal field theory, Hořava-Lifshitz and superrenormalizable gravity, display a feature known as dimensional flow or dimensional reduction, namely, the change of spacetime dimensionality with the scale ["t Hooft, 1993], [Carlip, 2009], [Calcagni, 2009].

\subsection{Quantum Fields and Gravity}

Quantum Mechanics has been used successfully to describe the physics of the small i.e. from atoms to quarks, their forces and interactions. Einstein's General Theory of Relativity [Weinberg, 1972], [Misner,Thorne,Wheeler,2000], which describes the physics of the very large, from the motion of planets in the solar system to the motion of galaxies in the Universe, that is, the gravitational interaction. General Relativity, or GR for short, is an excellent classical theory, it agrees with the classical tests of GR [Will, 2006] and it provides a beautiful geometric interpretation of gravity. However, GR is not renormalizable. This means among other things that, we cannot compute quantum corrections to the classical results, black hole thermodynamics cannot be understood in statistical terms (we cannot count states) and near the big bang, GR breaks down i.e. quantum effects dominate the evolution of the Universe and perhaps this could explain inflation. To solve these problems, physicist have tried to find a unified theory that can encompass all phenomena in all scales and, in the particular case of the gravitational interaction, a Quantum Theory of Gravity.

\subsection{Reduction of Couplings, Superintegrable Renormdynamics and Finite Quantum Field Theories}

The main goal of a unified description of interactions should be the understanding of the present day free parameters of the Standard Model (SM) in terms of a few fundamental ones, or in other words to achieve reduction of couplings at a more fundamental level. An impressive aspect of the renormdynamic integrals of motion (RIM) is that one can guarantee their validity to all-orders in perturbation theory by studying the uniqueness of the resulting relations at one-loop, as was proven in the early days of the programme of reduction of couplings [Zimmermann, 1985, [Oehme, Zimmermann, 1985]. Even more remarkable is the fact that it is possible to find RIM that guarantee finiteness to all-orders in perturbation theory [Lucchesi,Piguet,Sibold,1988], [Lucchesi,Zoupanos, 1997], [Piguet,Sibold,1986].

\subsection{Nambu - Poisson formulation of Renormdynamics}

In the case of several integrals of motion, $H_{n}, 1 \leq n \leq N$, we can formulate Renormdynamics as Nambu - Poisson dynamics (see e.g. [Makhaldiani, 2007])

$$
\dot{\varphi}(x)=\left[\varphi(x), H_{1}, H_{2}, \ldots, H_{N}\right]
$$

where $\varphi$ is an observable as a function of the coupling constants $x_{m}, 1 \leq m \leq M$.

In the case of Standard model [Weinberg, 1995], we have three coupling constants, $M=3$. 


\subsection{Hamiltonian extension of the Renormdynamics}

The renormdynamic motion equations

$$
\dot{g}_{n}=\beta_{n}(g), 1 \leq n \leq N
$$

can be presented as nonlinear part of a Hamiltonian system with linear part

$$
\dot{\Psi}_{n}=-\frac{\partial \beta_{m}}{\partial g_{n}} \Psi_{m},
$$

Hamiltonian and canonical Poisson bracket as

$$
H=\sum_{n=1}^{N} \beta(g)_{n} \Psi_{n},\left\{g_{n}, \Psi_{m}\right\}=\delta_{n m}
$$

In this extended version, we can define optimal control theory approach [Pontryagin, 1983] to the unified field theories. We can start from the unified value of the coupling constant, e.g. $\alpha^{-1}(M)=29.0 \ldots$ at the scale of unification $M$, put the aim to reach the SM scale with values of the coupling constants measured in experiments, and find optimal threshold corrections to the RD coefficients [Makhaldiani, 2010].

\subsection{Renormdynamic equation for effective action}

For connected vertex functions $\Gamma_{n},([]$.

$$
\begin{aligned}
& \Gamma_{n}\left(x_{1}, x_{2}, \ldots, x_{n} ; g, m, \mu\right)=Z^{n / 2}(\mu) \Gamma_{0 n}\left(x_{1}, x_{2}, \ldots, x_{n} ; g_{0}, m_{0}\right), \\
& \left(D-\frac{n}{2} \gamma\right) \Gamma_{n}(x ; g, m, \mu)=0
\end{aligned}
$$

For effective action $S_{q}$,

$$
\begin{aligned}
& \left(D-\frac{1}{2} \gamma \int d x \phi(x) \frac{\delta}{\delta \phi(x)}\right) S_{q}(\phi)=0 \\
& \left(D-\frac{1}{2} \gamma \phi \frac{\partial}{\partial \phi}\right) V(\phi)=0, V(\phi)=\left.S_{q}(\phi(x))\right|_{\phi(x)=\phi=c o n s t}
\end{aligned}
$$

where $V(\phi)$ is effective potential.

For the effective potential in the RD (conformal) fixed point, $\gamma(g)=\gamma\left(g_{c}\right) \equiv \gamma_{c}$ we have the following wave equation and corresponding (auto model) solution

$$
\begin{aligned}
& \left(\partial_{t}-\frac{\gamma_{c}}{2} \partial_{z}\right) V=0, \\
& V(\phi, \mu)=f(z+v t)=F\left(\frac{\phi}{\mu^{v}}\right), t=\ln \frac{\mu}{\mu_{0}}, z=\ln \frac{\phi}{\phi_{0}}, v=\frac{\gamma_{c}}{2} .
\end{aligned}
$$

\subsection{Renormdynamics of Observables}

In an axiomatic setting it was shown [Zimmermann, 1980] that the S-operator scales with the $\beta$-function

$$
\dot{S}=\beta(a) \partial_{a} S
$$


This can be understood as the renormdynamic equation for the S-operator. There is no contribution by $\gamma$-functions associated with anomalous dimensions of the fields.

If we take the solutions as the following expansion

$$
S=\sum_{n} g^{n} S_{n}
$$

for conformal theories: $\beta(a)=0$, we will have renormdynamic integrals of motion $S_{n}$. If we start from free fields, we will have usual perturation expansion: $n \geq 0$. If we start from nonperturbative, e.g. a soliton-like states, we will have also negative values for $n$.

We find the following exact form for the renormalization constant $Z$, [Makhaldiani, 1988]

$$
\begin{aligned}
& Z(a, \varepsilon)=1+Z_{1} \varepsilon^{-1}+\ldots+Z_{n} \varepsilon^{-n}+\ldots \\
& =1+\int^{a} d a \sum_{n \geq 1}\left(\frac{1}{\varepsilon} \frac{d Z_{1}}{d a}\right)^{n} \\
& =1+\int^{a} d a \frac{\frac{d Z_{1}}{d a}}{\varepsilon-\frac{d Z_{1}}{d a}} \\
& =1+\int^{a} d a \frac{\beta(a)}{\varepsilon a^{2}-\beta(a)} .
\end{aligned}
$$

In critical dimension $\varepsilon=0$, and

$$
\begin{aligned}
& 0 \leq Z(a, 0)=1-a \leq 1 \\
& 0 \leq a \leq 1, a_{b}=a(1-a) \Rightarrow \\
& a_{ \pm}=\frac{1 \pm \sqrt{1-4 a_{b}}}{2}, 0 \leq a_{b} \leq \frac{1}{4}
\end{aligned}
$$

These formal considerations are correct in the nonstandard (non-archimedean) analysis [Davis, 1977].

For any multiplicative renormalized quantity $A, A_{b}=Z(\varepsilon, a) A$,

$$
\begin{aligned}
& \dot{A}=-\gamma(\varepsilon, a) A, \gamma=\dot{Z} / Z, \\
& A=\exp \left(-\int^{a} d a \frac{\gamma(\varepsilon, a)}{-\varepsilon a+\beta(a)}\right),
\end{aligned}
$$

for $A=a, \gamma=\varepsilon-\beta(a) / a$.

\subsection{Dual symmetry}

The solutions for the observable values of the coupling constant $a_{ \pm}$, has the property

$$
a_{+} a_{-}=a_{b}
$$

which is similar with the Dirac's quantization

$$
\begin{aligned}
& \alpha_{e} \alpha_{m}=\frac{1}{4}=(4 \pi)^{2} a_{b}=e_{b}^{2}, e_{b}= \pm \frac{1}{2}, \\
& a_{b}=\frac{n^{2}}{64 \pi^{2}} \leq \frac{1}{4} \Rightarrow|n| \leq 12
\end{aligned}
$$

This condition gives the restriction on the charge, correspondingly, mass, size,..., of the cumulative quasiparticles.

We have a dual symmetry with respect to the change $a_{ \pm} \rightarrow a_{\mp}$, or $a \leftrightarrow Z$. If $a=a_{-}$is not small, we can expend with respect to $a_{+}=a_{b} / a=Z=1-a$. 


\subsection{Discrete renormdynamic interpretation}

If we consider $a_{b}$ as effective coupling constant at the n-th RD iteration, $a=a_{n}$, take $a_{b}=a_{n+1}$, we will have

$$
\begin{aligned}
& a_{n}=a_{n+1}=k a_{n}\left(1-a_{n}\right), \\
& \Delta a_{n}=a_{n+1}-a_{n}=a_{n}\left((k-1)-k a_{n}\right)=\left\{\begin{array}{rr}
>0, & a_{n}<(k-1) / k \\
<0, & a_{n}>(k-1) / k
\end{array}\right\}
\end{aligned}
$$

So, we have two unstable UV (stable IR) fixed points: $a=0$ and $a=1$ and one stable UV (unstable IR) fixed point $a_{s}=(k-1) / k$. When $k=1+\varepsilon, \varepsilon<<1, a_{s}=\varepsilon /(1+\varepsilon)$.

Another example of discrete dynamics without extra parameter $k$ is

$$
\begin{aligned}
& a_{n+1}=\sqrt{a_{n}\left(1-a_{n}\right)}=\gamma\left(a_{n}\right) a_{n} \leq \frac{1}{2}, \gamma\left(a_{n}\right)=\sqrt{a_{n}^{-1}-1} \geq 1, \\
& a_{n} \uparrow \frac{1}{2}
\end{aligned}
$$

\subsection{Renormdynamic functions (RDF)}

We will call RDF functions $g_{n}=f_{n}(t)$, which are solutions of the RD motion equations

$$
\dot{g}_{n}=\beta_{n}(g), 1 \leq n \leq N .
$$

In the simplest case of one coupling constant, the function $g=f(t)$, is constant $g=g_{c}$ when $\beta\left(g_{c}\right)=0$, or is invertible (monotone). Indeed,

$$
\dot{g}=f^{\prime}(t)=f^{\prime}\left(f^{-1}(g)\right)=\beta(g) .
$$

Each monotone interval ends by UV and IR fixed points and describes corresponding phase of the system.

\subsection{Minimal Supersymmetric Standard Model (MSSM) and Higgs Particles}

The LHC has discovered a scalar with mass around $125 \mathrm{GeV}$ which resembles the Standard Model (SM) Higgs boson [Aad et al. ATLAS Collaboration, 2012], [Chatrchyan et al. CMS Collaboration, 2012]. Since the MSSM predicts a light Higgs boson below $130 \mathrm{GeV}$ [MSSMHiggs, 1991-1993], the discovery of such a $125 \mathrm{GeV}$ Higgs boson may be the second hint of low energy supersymmetry (SUSY). First hint was the possibility of unification of the coupling constants, the property beyond the SM.

At an $e^{+} e^{-}$collider, the Higgs-strahlung process $e^{+} e^{-} \Rightarrow Z h$ is the dominant production channel for the Higgs boson, for which the $\mathrm{Zh}$ events can be inclusively detected by tagging a leptonic $\mathrm{Z}$ decay without assuming the Higgs decay mode. For a center-of-mass energy of $240-250 \mathrm{GeV}$ and an integrated luminosity of $500 \mathrm{fb}^{-1}$, an $e^{+} e^{-}$collider can produce about $O\left(10^{5}\right)$ Higgs bosons per year and allow for measuring the Higgs couplings at percent level [Peskin, 2012], [B]ondel et al_2013], which may be able to unravel the SUSY effects in this production.

As a feasible option, the $\gamma \gamma$ collision can be achieved through the backward Compton scattering of laser light against high-energy electrons at a linear $e^{+} e^{-}$collider. At such a $\gamma \gamma$ collision the 
Higgs boson can be singly produced via the loop process $\gamma \gamma \Rightarrow h$. This process is demonstrated to be sensitive to the new charged SUSY particles. So the photon collider will be an ideal place to investigate the anomalous $h \gamma \gamma$ coupling. At the $\gamma \gamma$ collider, the Higgs partial width $\Gamma_{\gamma \gamma}$ can be measured with an accuracy of about $2 \%$. Besides, the CP property of the Higgs boson can be measured using the photon polarizations. The single production of SUSY Higgs bosons through $\gamma \gamma$ fusion has been calculated in [Bae et al.2000-2008] .

\subsection{Dark Matter}

Evidently, about 84 percent of the matter in the universe does not absorb or emit light. "Dark matter," as it is called, cannot be seen directly, and it hasn't yet been detected by indirect means, either. Instead, dark matter's existence and properties are inferred from its gravitational effects on visible matter, radiation and the structure of the universe. This shadowy substance is thought to pervade the outskirts of galaxies, and may be composed of "weakly interacting massive particles," or WIMPs. The mass $m$ of the observable part of the universe is dominated by nucleons: $m=N_{n} m_{n}$. If the mass of the dark matter is dominated by the mass $m_{D}$ of a dark matter particle: $M=N_{D} m_{D}$, than

$$
\frac{M}{m}=\frac{N_{D}}{N_{n}} \frac{m_{D}}{m_{n}}=\frac{84}{16}=\frac{21}{4}=5.25
$$

If we take $N_{D}=N_{n}$ than we predect: $m_{D}=5.25 m_{n}=5.25 \times 0.94=4.93 \simeq 5 \mathrm{GeV}$. If we take $m_{D}=41 \mathrm{GeV}$, than $N_{D} \simeq N_{n} / 8$. If we take $m_{D}=2.4 \mathrm{TeV}$, than $N_{D} \simeq N_{n} / 60$. If we take $m_{D}=0.33$ $\mathrm{GeV}$ of the valence quark value, than $N_{D} \simeq 15 N_{n}$.

\subsection{Candidates in dark matter particle}

Many new physics models contain massive neutral particles whose stability is protected by certain exact or approximate symmetries, and thus could serve as DM particles. Antimatter is a good way to look for dark matter. Middle way for the mass scale of the DMP is $40 \mathrm{GeV}$. DMP with mass less than $40 \mathrm{GeV}$ we will call light candidates, DMP with mass more than $40 \mathrm{GeV}$ we will call heavy candidates.

According to the SM of cosmology, the critical density of matter in the flat universe is

$$
\rho_{c}=\frac{3 H^{2}}{4 \pi G}=2 \times 10^{-29} \frac{\mathrm{g}}{\mathrm{cm}^{3}},
$$

The density of baryon matter is

$$
\rho_{b}=5 \times 10^{-31} \frac{\mathrm{g}}{\mathrm{cm}^{3}},
$$

If the number of the dark matter particles (DMP, $D_{m}$ ) of mass $m$, is k-times more than baryon number than

$$
\frac{m}{m_{p}}=\frac{\rho_{c}}{k \rho_{b}}=40 k^{-1}
$$

so, for $k=3$ we have

$$
\frac{m}{m_{p}} \gtrsim 13=\alpha_{\pi N}
$$


for $k=1 / 3$

$$
m=120 m_{p} \lesssim 126 G e V=m_{H}
$$

If the Universe baryon number is zero, than DMP with mass $m=120 \mathrm{GeV}$ must have baryon number $n_{B}=-3$ and the Higgs particle with mass $m_{H}=126 \mathrm{GeV}$ may decay as $H \rightarrow D_{m}+3 n$, with low energy $(1 \mathrm{GeV})$ neutrons. More datal picture of the decay is

$$
H \rightarrow H_{1 / 2}+n, H_{1 / 2} \rightarrow H_{1}+n, H_{1} \rightarrow H_{3 / 2}+n
$$

In this case the spin of $D_{m}$ is $3 / 2$.

In the case of $k=1, m=40 m_{p}=37.6 \mathrm{GeV}$. We have seen that at the scale $41 \mathrm{GeV}$ the weak coupling constant $\alpha_{2}^{-1}=29.0$ and can be unified with electromagnetic and strong coupling constants. The case with $m / m_{p} \simeq 4$ or 3 corresponds to the (anti-)helium, in the superfluid-dark matter phase.

\subsection{Strangelet}

A strangelet is a hypothetical particle consisting of a bound state of roughly equal numbers of up, down, and strange quarks. Its size would be a minimum of a few femtometers across (with the mass of a light nucleus). Once the size becomes macroscopic (on the order of metres across), such an object is usually called a quark star or "strange star" rather than a strangelet. An equivalent description is that a strangelet is a small fragment of strange matter. The term "strangelet" originates with E. Farhi and R. Jaffe [Farhi, Jaffe, 1984]. Strangelets have been suggested as a dark matter candidate [Witten, 1984].

According to the "strange matter hypothesis" of Bodmer [Bodmer, 197]] and Witten [Witten, 1984], when a large enough number of quarks are collected together, the lowest energy state is one which has roughly equal numbers of up, down, and strange quarks, namely a strangelet. This stability would occur because of the Pauli exclusion principle; having three types of quarks, rather than two as in normal nuclear matter, allows more quarks to be placed in lower energy levels. The known particles with strange quarks are unstable because the strange quark is heavier than the up and down quarks, so strange particles, such as the Lambda particle, which contains an up, down, and strange quark, always lose their strangeness, by decaying via the weak interaction to lighter particles containing only up and down quarks. But states with a larger number of quarks might not suffer from this instability.

\subsection{A Scenario for Centauro Events}

A primary cosmic-ray particle interacts with air nuclei and produce a fireball, which decays on neutral pions: $\gamma \gamma \Rightarrow \pi^{0}$, by inverse mein decay channel, and/or $\gamma \rightarrow \rho^{0} \Rightarrow \pi^{+} \pi^{-}$, by vector meson dominance.

\subsection{Density of states description of the statistical systems}

If we take the trace of the transition amplitude,

$$
\sum_{s_{n}}<s_{n}|U(t)| s_{n}>=\sum_{n, m}<s_{n} \mid E_{m}>
$$




$$
\begin{aligned}
& <E_{m} \mid s_{n}>\exp \left(-\frac{i}{\hbar} E_{m}\right) \\
& =\sum_{E_{m}} N\left(E_{m}\right) e^{-\beta E_{m}}=\int_{0}^{\infty} d E \rho(E) e^{-\beta E} \\
& =\rho\left(E_{c}\right) e^{-\beta E_{c}}=e^{-\beta F}=Z(\beta), \\
& F=E_{c}-T S, \quad S=\ln \rho\left(E_{c}\right), \\
& \partial S / \partial E=T^{-1}=\beta,
\end{aligned}
$$

for density of states we will have

$$
\begin{aligned}
& \rho(E)=\sum_{E_{m}} N\left(E_{M}\right) \delta\left(E-E_{M}\right) \\
& =\sum_{m} \delta\left(E-E_{m}\right)=\operatorname{tr}(E-\hat{H}) \\
& =\frac{1}{2 \pi i \hbar} \int_{-\infty}^{+\infty} d t e^{\frac{i}{\hbar} t E} \operatorname{tr}\left(e^{-\frac{i}{\hbar} t \hat{H}}\right)
\end{aligned}
$$

Note, that in this type of expressions, we clearly see the origin of the energy - time uncertainty relation, $\Delta E \Delta t \sim 2 \pi \hbar$.

If there are besides of the energy also other integrals of motion, $N_{1}, \ldots, N_{k}$,

$$
\begin{aligned}
& \rho\left(E, N_{1}, \ldots, N_{k}\right)=\operatorname{tr}\left(\boldsymbol{\delta}(E-\hat{H}) \boldsymbol{\delta}\left(N_{1}-\hat{N}_{1}\right)\right. \\
& \left.\ldots \delta\left(N_{k}-\hat{N}_{k}\right)\right)
\end{aligned}
$$

\subsection{High energy, baryon number and temperature asymptotics}

If one knows the zero temperature dynamics, i.e. the complete spectrum of the hamiltonian, then the quantum statistical mechanics just requires an additional statistical summation

$$
Z=\operatorname{tr}\left(e^{-\beta H}\right) \text {. }
$$

In practice, the complete non-perturbative spectrum of $\mathrm{H}$ is unknown so one must resort to perturbative methods, such as the Matsubara approach, that typically entangle the zero temperature dynamics from the quantum statistical mechanics. Let us take the hamiltonian form of the functional integral

$$
t r e^{-\frac{i}{\hbar} t \hat{H}}=\int_{x(0)=x(t)} \frac{d x d p}{2 \pi \hbar} e^{\frac{i}{\hbar} \int_{0}^{t}(p \dot{x}-H)}
$$

For higher energy, small time and static approximation we have

$$
\begin{aligned}
& \rho(E)=\frac{1}{2 \pi i \hbar} \int_{-\infty}^{+\infty} d t e^{\frac{i}{\hbar} t E} \operatorname{tr}\left(e^{-\frac{i}{\hbar} t \hat{H}}\right) \\
& =\frac{1}{2 \pi i \hbar} \int_{-\infty}^{+\infty} d t e^{\frac{i}{\hbar} t E} \int \frac{d x d p}{2 \pi \hbar} e^{-\frac{i}{\hbar} t H(p, x)} \\
& =\int \frac{d x d p}{2 \pi \hbar} \delta(E-H(p, x)) \\
& =\int \frac{d x}{2 \pi \hbar} \frac{2 m}{\sqrt{2 m(E-V(x))}}
\end{aligned}
$$

The number of states

$$
N(E)=\int d E \rho(E)=\int_{H(p, x)=E} \frac{d x d p}{2 \pi \hbar}
$$




\subsection{Exactly solvable model with maximal temperature}

At high energy and temperature, we have classical statistical description

$$
\begin{aligned}
& Z(\beta)=\int \frac{d^{D} x d^{D} p}{(2 \pi)^{D}} e^{-\beta H(p, x)} \\
& =\left(\frac{m}{2 \pi \beta}\right)^{D / 2} \int d^{D} x e^{-\beta V(x)}
\end{aligned}
$$

For potential of the form

$$
\begin{aligned}
V(x) & =0,0 \leq|x| \leq a, \\
& =b \cdot \ln \frac{|x|}{a},|x|>a,
\end{aligned}
$$

we have

$$
Z(\beta)=\left(\frac{m}{2 \pi \beta}\right)^{D / 2} \frac{\Omega_{D}}{D} \frac{a^{D} \beta b}{\beta b-D}
$$

So the temperature of the system is restricted by condition

$$
T=\beta^{-1}<b / D \equiv T_{H}
$$

Statistical energy of the system is

$$
\begin{aligned}
& E=E_{c}=-\frac{\partial \ln Z(\beta)}{\partial \beta} \\
& =\frac{D / 2-1}{\beta}+\frac{1}{\beta-D / b}
\end{aligned}
$$

The case when $E=0$ corresponds to a self supporting, non expending, no collapsing state of the system at the temperature

$$
\begin{aligned}
& \beta_{N}=\frac{2}{b}\left(\frac{D}{2}-1\right)=\frac{D}{b}-\frac{2}{b}<\beta_{H} \\
& T_{N}=\frac{b}{D-2}=\frac{T_{H}}{1-2 / D}>T_{H}
\end{aligned}
$$

The normal temperature is positive, corresponds to the stable state, for $D>2$. It decrees when dimension increase, from infinity to zero. So it is easy (easier)to crate higher dimensional normal states. The volume of the system is

$$
\begin{aligned}
& V_{c}=\frac{\int d x d p V e^{-\beta H}}{\int d x d p e^{-\beta H}} \\
& =\frac{\Omega_{D}}{D} \frac{\int_{0}^{a} d x x^{2 D-1}+\int_{a}^{\infty} d x x^{2 D-1}\left(\frac{a}{x}\right)^{b \beta}}{Z(\beta)} \\
& =\frac{\Omega_{D}}{D} a^{D} \frac{b \beta-D}{2(b \beta-2 D)}, V_{N}=\frac{V(a)}{D+2}
\end{aligned}
$$

The volume has positive value, for $T>T_{2}=b / D=T_{H}$ or $T<T_{1}=b / 2 D, T_{N}>T_{2}$.

This Normal state can not be reached by rising continually temperature of the system. In the corresponding realistic models, e.g. for a heavy nucleus, we can obtain such a state in high energy collisions.To this kind of physical states maybe ascribed ball lightning. Our model has one of the main observable properties of the ball lightning, the normal states stable for temperatures $T>T_{N}$, decays for lower temperatures, when $T \leq T_{N}$. Probably famous Tunguska Event was a Big ball lightning explosion. This model maybe also useful as a model of the Centauro fireball. 


\subsection{Ridges}

In nucleus-nucleus collisions, ridges occur in the di-hadron correlations as structures that are elongated in pseudorapidity difference $\Delta \eta=\eta_{1}-\eta_{2}$, and peak on the near $\Delta \phi \sim 0$ and away sides $\Delta \phi \sim \pi$, where $\Delta \phi$ is the azimuthal angle difference between hadron pairs.

\subsection{9 n-field effective action and quasi particle spectrum}

The $O(N)-\sigma$ model is given by the following action functional (in euclidian) space(time) (see e.g. [Novikov et al, 1984])

$$
\begin{aligned}
& A=\frac{N}{2 g} \int d^{D} x\left(\partial_{\mu} n_{a}\right)^{2}, \\
& \sum_{a=1}^{N} n_{a}^{2} \equiv n^{2}=1 .
\end{aligned}
$$

After scaling of the field variable and including the constraint using the Lagrange multiplier, for GF we will have

$$
\begin{aligned}
& Z_{J}=\int d n_{a} d \alpha \exp (-S(n, \alpha)+j \cdot n), \\
& S=\int d x\left(\frac{1}{2}\left((\partial n)^{2}+\alpha(x)\left(n^{2}-\frac{N}{g}\right)\right)\right), \\
& Z_{J}=\int d \alpha e^{-S_{e f f}+\frac{1}{2} J^{a} \cdot D^{-1} \cdot J^{a}} \\
& S_{e f f}=\frac{N}{2} \operatorname{trln} D-\frac{N}{2 g} \int d x \alpha, \\
& D(\alpha)=-\partial^{2}+\alpha
\end{aligned}
$$

If the motion equation for $\alpha$ has a classical solution $\alpha_{c}=m^{2}$, we can expend the effective action in quantum fluctuations, $\alpha=m^{2}+\alpha_{q}$,

$$
\begin{aligned}
& S_{e f f}=\frac{N}{2} \operatorname{trln}\left(-\partial^{2}+m^{2}\right) \\
& -\frac{N}{2 g} \int d x m^{2}-\frac{N}{2 g} \int d x \alpha_{q} \\
& +\frac{N}{2} \sum_{k \geq 1} \frac{(-1)^{k+1}}{k} \operatorname{tr}\left(D^{-1} \alpha_{q}\right)^{k} .
\end{aligned}
$$

When $\alpha_{c}$ is classical solution, linear terms in the expansion compensates each other,

$$
\begin{aligned}
& \frac{1}{g} \int d x \alpha_{q}=\operatorname{tr} \frac{1}{-\partial^{2}+m^{2}} \alpha_{q} \\
& =\int d x<x\left|\frac{1}{-\partial^{2}+m^{2}}\right| x>\alpha_{q}(x) \\
& =<0\left|\frac{1}{-\partial^{2}+m^{2}}\right| 0>\int d x \alpha_{q},
\end{aligned}
$$

so, in $D=2$,

$$
\frac{1}{g}=<0\left|\frac{1}{-\partial^{2}+m^{2}}\right| 0>=\int \frac{d^{D} p}{(2 \pi)^{D}} \frac{1}{p^{2}+m^{2}}
$$




$$
\begin{aligned}
& =\frac{1}{2 \pi} \int_{0}^{\infty} \frac{d p p}{p^{2}+m^{2}} \Rightarrow \frac{1}{2 \pi} \int_{0}^{\Lambda} \frac{d p p}{p^{2}+m^{2}} \\
& =\frac{1}{4 \pi} \ln \frac{\Lambda^{2}}{m^{2}}, \\
& g \Rightarrow g(\Lambda)=\frac{2 \pi}{\ln \frac{\Lambda}{m}}-
\end{aligned}
$$

is bare coupling constant, the running coupling constant is

$$
g(\mu)=\frac{2 \pi}{\ln \frac{\mu}{m}}=\frac{g_{0}}{1+\frac{g_{0}}{2 \pi} \ln \frac{\mu}{\mu_{0}}} ;
$$

the model is asymptotically free; the mass depends on the coupling constant non analytically,

$$
m=\mu \exp \left(-\frac{2 \pi}{g(\mu)}\right)
$$

\subsection{Neutral quasiparticle $\alpha$}

The quadratic therm in the effective action generates the propagator of the new $\alpha$-quasiparticle,

$$
\begin{aligned}
& S_{e f_{2}}=-\frac{N}{4} \operatorname{tr}\left(\frac{1}{-\partial^{2}+m^{2}} \alpha \frac{1}{-\partial^{2}+m^{2}} \alpha\right) \\
& =-\frac{N}{4} \int d^{2} x d^{2} y<x\left|\frac{1}{-\partial^{2}+m^{2}}\right| y> \\
& \alpha(y)<y\left|\frac{1}{-\partial^{2}+m^{2}}\right| x>\alpha(x)=\frac{1}{2} \alpha \cdot V_{2} \cdot \alpha \\
& =\frac{1}{2} \int d^{2} x d^{2} y \alpha(x) V_{2}(x-y) \alpha(y) \\
& =\frac{1}{2} \int d^{2} p|\alpha(p)|^{2} V_{2}(p), V_{2}(p)=-\frac{N}{2} V(p) \text {, } \\
& V(p)=\int d^{2} x e^{-i x p} \frac{1}{(2 \pi)^{4}} \int d^{2} q d^{2} r e^{i x(q+r)} \\
& \frac{1}{q^{2}+m^{2}} \frac{1}{r^{2}+m^{2}}=\frac{1}{(2 \pi)^{2}} \\
& \int d^{2} q \frac{1}{q^{2}+m^{2}} \frac{1}{(p+q)^{2}+m^{2}}=\frac{1}{(2 \pi)^{2}} \\
& \int_{0}^{1} d x \int d^{2} q \frac{1}{\left(q^{2}+x(1-x) p^{2}+m^{2}\right)^{2}} \\
& =\int d x I(p, x), I(p, x)= \\
& \frac{1}{(2 \pi)^{2}} \int d^{2} q \frac{1}{\left(q^{2}+x(1-x) p^{2}+m^{2}\right)^{2}} \\
& =\frac{1}{4 \pi} \int_{0}^{\infty} d u \frac{1}{(u+a(p, x))^{2}}=\frac{1}{4 \pi} \frac{1}{a(p, x)}, \\
& a(p, x)=x(1-x) p^{2}+m^{2} \text {, } \\
& V(p)=\frac{1}{4 \pi} \int_{0}^{1} \frac{d x}{a(p, x)}, V(0)=\frac{1}{4 \pi m^{2}} \\
& V(p)=\frac{1}{2 \pi p^{2}} \frac{1}{x_{1}-x_{2}} \ln \left|\frac{x_{1}}{x_{2}}\right| \\
& =\frac{1}{2 \pi p^{2}} \frac{1}{\sqrt{1+\frac{4 m^{2}}{p^{2}}}} \ln \frac{\sqrt{1+\frac{4 m^{2}}{p^{2}}}+1}{\sqrt{1+\frac{4 m^{2}}{p^{2}}}-1}
\end{aligned}
$$


where $x_{1}$ and $x_{2}$ are roots of $a(p, x)$

$$
x_{ \pm}=\frac{1 \pm \sqrt{1+\frac{4 m^{2}}{p^{2}}}}{2} .
$$

Note that when, $p^{2}=m^{2}$, the roots take Golden ratio values,

$$
x_{1}=\frac{\sqrt{5}+1}{2}
$$

The $n$ - field propagator has pole at the mass shell, $p^{2}=-m^{2}$; the quasi particle propagator has cut at $-4 m^{2}<R e p^{2}<0$.

For $\left|p^{2}\right| \gg 4 m^{2}$

$$
\begin{aligned}
& V(p) \simeq Z(p) \frac{1}{p^{2}+2 m^{2}}, \\
& Z(p)=\frac{1}{2 \pi} \ln \frac{p^{2}}{m^{2}}
\end{aligned}
$$

for $0<p^{2} \ll 4 m^{2}$,

$$
V(p) \simeq \frac{1}{\pi} \frac{1}{4 m^{2}+p^{2}}, Z=\frac{1}{\pi}
$$

First of all, we need to make a scaling transformation of the field $\alpha$,

$$
\alpha(p) \rightarrow(N Z(p))^{-1 / 2} \alpha
$$

to give a sense to the kinetic term of the action. This transformation suppress higher order terms in action, for high values of $N$. Then, for high and low values of $p^{2}$ the term is like of the source term of the particle field with mass $M_{1}=\sqrt{2} m$ and $M_{2}=2 m$, correspondingly,

$$
\begin{aligned}
& -\frac{1}{2} \int d p|\alpha(p)|^{2} \frac{1}{p^{2}+M^{2}} \Rightarrow \\
& \int d x\left(\frac{1}{2}\left((\partial \phi)^{2}+M^{2} \phi^{2}\right)\right. \\
& +\alpha(x) \varphi(x)),
\end{aligned}
$$

so in this region of scales $\alpha$-field interacts with the fundamental color field of mass $m, n^{a}, a=$ $1, \ldots, N$, and acts as a source of the neutral hadron field of mass $M, \phi$. Note that, $M_{2}^{2}-M_{1}^{2}=2 m^{2}$, and on the way from small distance to large one, the neutral quasi-particle from strongly coupled bound state of two colored particles become weakly coupled.

\subsection{D-dimensional case}

In D dimension, the coupling constant has mass dimension, $g_{D}=\mu^{2-D} g_{0}$, so

$$
\begin{aligned}
& \frac{1}{g_{0}}=\mu^{2-D} \int \frac{d^{D} p}{(2 \pi)^{D}} \frac{1}{p^{2}+m^{2}} \\
& =\frac{\Omega_{D}}{(2 \pi)^{D}} \mu^{2-D} \int_{0}^{\infty} \frac{d p p^{D-1}}{p^{2}+m^{2}}
\end{aligned}
$$




$$
\begin{aligned}
& =\frac{\Omega_{D}}{2(2 \pi)^{D}}\left(\frac{m}{\mu}\right)^{D-2} \Gamma\left(1-\frac{D}{2}\right) \Gamma\left(\frac{D}{2}\right), \\
& g_{0}(\mu)=c(D)\left(\frac{\mu}{m}\right)^{D-2}, \\
& c(D)=\frac{(4 \pi)^{D / 2}}{\Gamma(1-D / 2)}
\end{aligned}
$$

It is interesting to obtain the previous expression for running coupling constant in critical dimension with momentum cutoff. For this we take $D=2-2 \varepsilon$ and expend in $\varepsilon$ the inverse value of the coupling constant

$$
\begin{aligned}
& \frac{1}{g_{0}}=\Gamma(1-D / 2)(4 \pi)^{-D / 2}\left(\frac{\mu}{m}\right)^{2-D} \\
& =\frac{1}{\varepsilon} \Gamma(1+\varepsilon) \frac{1}{4 \pi}(4 \pi)^{\varepsilon}\left(\frac{\mu}{m}\right)^{2 \varepsilon} \\
& =\frac{1}{\varepsilon}(1-\gamma \varepsilon)(1+\varepsilon \ln (4 \pi))\left(1+2 \varepsilon \ln \frac{\mu}{m}\right) \frac{1}{4 \pi} \\
& =C+\frac{1}{2 \pi} \ln \frac{\mu}{m}, \\
& \frac{1-C g_{0}}{g_{0}}=\frac{Z}{g_{0}}=\frac{1}{g(\mu)}=\frac{1}{2 \pi} \ln \frac{\mu}{m}, \\
& C=\frac{1}{\varepsilon}-\gamma+\ln (4 \pi)
\end{aligned}
$$

\subsection{Hadronization in Skyrme and other models of QCD}

Model independent predictions of semiclassical - 1/N expansion of the $S U(N) Q C D$ includes the following relations

$$
\mu_{P}-\mu_{N}=\mu_{P \Delta} / \sqrt{2}, g_{\pi N \Delta}=3 / 2 g_{\pi N} \Rightarrow \alpha_{\pi N \Delta}=(3 / 2)^{2} \alpha_{\pi N}=29.25
$$

which are valid within few percent approximation. So we may take $\alpha_{\pi N \Delta}=p=29$ and consider p-adic convergence of the $\alpha_{\pi N \Delta}$ expansion. For two flavors the ground state barons have spin isospin $J=I=1 / 2$ for any odd $N$. Both in the quark and skyrmion models the short range interaction between two nucleons is repulsive but the short range interaction between two $\Lambda^{0}$ is attractive. So, a bound state $H$ of two $\Lambda^{0}$ with binding energy of $110-200 \mathrm{MeV}$ was predicted [Balachandra,Kurkcuoglu,Rojas,2002].

\subsection{Geometry of dynamics and confinement}

Let us consider a particle with mass $m$ and energy $E$ in the potential $V(x)$,

$$
\begin{aligned}
& E=\frac{m}{2}\left(\frac{d x_{n}}{d t}\right)^{2}+V(x) \Rightarrow \\
& d s^{2}=c(x)^{2} d t^{2}-d x_{n}^{2}=0 \\
& c(x)=\sqrt{\frac{2}{m}(E-V(x))} .
\end{aligned}
$$

So, we can interpret this dynamics as lights with variable velocity $c(x)$. For rising potentials, when $V(x)=E, c(x)=0$ and the particle and corresponding light are confined. 


\subsection{Constraints from CMB on NcSM}

Modern cosmology has now emerged as a testing ground for theories beyond the standard model of particle physics. In 1992, the Cosmic Background Explorer (COBE) satellite detected anisotropies in the CMB radiation, which led to the conclusion that the early universe was not smooth: there were small density perturbations in the photon-baryon fluid before they decoupled from each other. Quantum corrections to the inflaton field generate perturbations in the metric and these perturbations could have been carried over to the photon-baryon fluid as density perturbations. We then observe them today in the distribution of large scale structure and anisotropies in the CMB radiation.

Primordial metric fluctuations can be decomposed into scalar, vector and second rank tensor fluctuations according to their transformation properties under spatial rotations [Brandenberger,2004]. They evolve independently in a linear theory. Scalar perturbations are most important as they couple to matter inhomogeneities. Vector perturbations are not important as they decay away in an expanding background cosmology. Tensor perturbations are less important than scalar ones, they do not couple to matter inhomogeneities at linear order.

\subsection{Einstein-Maxwell-Dilaton Theory and Coupling Constants}

Charged black holes in four-dimensional Einstein-Maxwell-Dilaton (EMD) gravity exhibit different features depending on the value of the dilaton coupling constant $a$ entering the Maxwell term in the Lagrangian,

$$
L=R-2 \Lambda-2(\partial \varphi)^{2}-\exp (-2 a \varphi) F^{2}
$$

Let us assume the static ansatz for the metric and the Maxwell one-form:

$$
\begin{aligned}
& d s^{2}=-e^{-2 \delta} N d t^{2}+N^{-1} d r^{2}+R^{2} d \Sigma_{k}^{2} \\
& A=-f d t-P \cos \vartheta d \varphi
\end{aligned}
$$

where $P$ is the magnetic charge and metric on the 2-space maybe spherical, flat or hyperbolic: $k=1,0,-1$

$$
d \Sigma_{k}^{2}=\left\{\begin{array}{cc}
d \theta^{2}+\sin ^{2} \theta d \varphi^{2}, & k=1 \\
d \theta^{2}+\vartheta^{2} d \varphi^{2}, & k=0 \\
d \theta^{2}+\sinh ^{2} \theta d \varphi^{2}, & k=-1
\end{array}\right.
$$

the functions $f, \varphi, N, R$ and $\delta$ depend on the radial variable $r$ only

\subsection{Geometric flows}

Geometric flows (GF), and, in particular, Ricci flows, are interesting in their own right. In physics they originally appeared in off-critical string theory via the renormalization-group equations of two-dimensional non-linear sigma models, where the evolution of the metric under the Ricci flow equations provides the running of the bulk coupling to lowest order in perturbation theory (see [Eriedan,1980] for the original result). In this context, the renormalization-group time is provided by the logarithmic length scale of the world-sheet, but in some cases it can also assume 
the role of genuine time, describing real-time evolution in string theory in regimes where the friction due to the motion of the dilaton effectively reduces the second-order evolution equations to the first-order renormalization-group flow equations [Schmidhuber,Tseytlin, 1994], Bakas et al,2007]. In mathematics GF play a crucial role in implementing Hamilton's program for proving Poincar's and Thurston's conjectures [Hamilton,1982], [Perelman,2002-2003] (but see also [Morgan,2007] and references therein).

\subsection{Main flow}

Main flows (MF) we call the following generalizationof the Geometric flows. MF is characterised by a regular matrix $G_{m n}$. When this matrix is symmetric, MF reduce to GF. Let us consider a point particle system with configuration space $\Re$ and a system of local coordinates $q_{n}$ that describe its physical degrees of freedom. Let us consider the following functional $E$

$$
\begin{aligned}
& E=\frac{1}{2} \int d t\left(\dot{x}_{n}-\frac{\partial W}{\partial x_{k}} M_{k n}\right) M^{n m}\left(\dot{x}_{m}-M_{m l} \frac{\partial W}{\partial x_{l}}\right)=H-\triangle W \\
& H=\frac{1}{2} \int d t\left(\dot{x}_{n} M^{n m} \dot{x}_{m}+\frac{\partial W}{\partial x_{k}} M_{k l} \frac{\partial W}{\partial x_{l}}\right), \triangle W=\int_{t_{i}}^{t_{f}} d t \dot{x}_{n} \frac{\partial W}{\partial x_{n}}=W_{f}-W_{i}, \\
& W_{i f}=W\left(x\left(t_{i f}\right)\right), M_{n k} M^{k l}=\delta_{n}^{l}
\end{aligned}
$$

which corresponds to a point particle system with configuration space $\Re$ and a local coordinates $q_{n}$ that describe its physical degrees of freedom.

Note that in the kinetic part of the energy $H$

$$
\frac{1}{2} \int d t \dot{x}_{n} M^{n m} \dot{x}_{m}
$$

gives contribution the symmetric part $M_{s}$ of the matrix $M=M_{s}+M_{a}$ and in the potential part of the energy

$$
\frac{1}{2} \int d t \frac{\partial W}{\partial x_{k}} M_{k l} \frac{\partial W}{\partial x_{l}}
$$

gives contribution the symmetric part of the inverse function $M_{s}^{-1}$. We have the following relation

$$
M M^{-1}=M_{s} M_{s}^{-1}+M_{s} M_{a}^{-1}+M_{a} M_{s}^{-1}+M_{a} M_{a}^{-1}=I
$$

Which in the symmetric and antisymmetric cases reduce correspondingly to

$$
M_{a}=0 \Rightarrow M_{s} M_{s}^{-1}=I ; M_{s}=0 \Rightarrow M_{a} M_{a}^{-1}=I
$$

For antisymmetric $M, H=0, E=-\triangle W$. For symmetric and positive $M$, the functional $E \geq 0$ is the Liapunov's functional, with its minimum on the solutions of the following motion equation (ME)

$$
\dot{x}_{m}-M_{m l} \frac{\partial W}{\partial x_{l}}=0, H=\triangle W \geq 0 .
$$

For antisymmetric $M, \mathrm{ME}$ is the Hamiltonian ME of the following Lagrangian

$$
L=f_{n}(x) \dot{x}_{n}-W(x),
$$


with ME

$$
\begin{aligned}
& \dot{x}_{n}=M_{m l} \frac{\partial W}{\partial x_{l}}, \\
& M_{n m}=F_{n m}^{-1}, F_{n m}=\frac{\partial f_{n}}{\partial x_{m}}-\frac{\partial f_{m}}{\partial x_{n}}
\end{aligned}
$$

On the solutions of the ME, $E=H=\triangle W=0$.

\subsection{Ricci flow}

In Hamilton's Ricci flow (RF) [Hamilton,1982], the time evolution of the metric is proportional to the Ricci tensor,

$$
\dot{g}_{n m}=-2 R_{n m}
$$

and yields a forced diffusion equation for the curvature; i.e., the scalar curvature evolves as

$$
\dot{R}=\triangle R+2 R^{2}
$$

\subsection{Skyrmions}

The skyrmion is a hypothetical particle related originally to baryons. Skyrmions as topological objects are also important in solid state physics, especially in the emerging technology of spintronics. A two-dimensional skyrmion, as a topological object, is formed, e.g., from a $3 \mathrm{~d}$ effective-spin "hedgehog" (in the field of micromagnetics: out of a so-called "Bloch point" singularity of homotopy degree +1 ) by a stereographic projection, whereby the positive northpole spin is mapped onto a far-off edge circle of a 2d-disk, while the negative southpole spin is mapped onto the center of the disk.

In field theory, skyrmions are homotopically non-trivial classical solutions of a nonlinear sigma model with a non-trivial target manifold topology - hence, they are topological solitons. In chiral models of mesons, a topological term can be added to the chiral Lagrangian, whose integral depends only upon the homotopy class; this results in superselection sectors in the quantised model. A skyrmion can be approximated by a soliton of the Sine-Gordon equation; after quantisation by the Bethe ansatz or otherwise, it turns into a fermion interacting according to the massive Thirring model.

Skyrmions have been reported, but not conclusively proven, to be in Bose-Einstein condensates, [AI Khawaja,Stoof,200]] superconductors, [Baskaran,201] thin magnetic films [Kiselev et al,201] and also chiral nematic liquid crystals [Fukuda,Žumer,2011]. One particular form of the skyrmions is found in magnetic materials that break the inversion symmetry and where the DzyaloshinskiiMoriya interaction plays an important role. They form "domains" as small as a $1 \mathrm{~nm}$ (e.g. in Fe on $\operatorname{Ir}(111)$ [Heinze et al,201]). The small size of magnetic skyrmions makes them a good candidate for future data storage solutions. Physicists at the University of Hamburg have managed to read and write skyrmions using scanning tunneling microscopy [Romming et al,2013]. 


\subsection{Neutron-Antineutron Oscillations}

The neutron-antineutron oscillations are of great interest because their observation would allow to test very accurately the most fundamental CPT-symmetry, where $\mathrm{C}$ is charge conjugation, $\mathrm{P}$ is inversion of space and $\mathrm{T}$ is reversal of time. The three discrete symmetries $\mathrm{C}, \mathrm{P}, \mathrm{T}$ were established soon after discovery of Quantum Mechanics. C-symmetry or in other words symmetry between particles and corresponding antiparticles was introduced in 1932 when the existence of positron (the antiparticle of electron) was predicted by Paul Dirac and discovered by Carl Anderson. Particles which are identical with their antiparticles are called genuinely neutral. Such is the photon - the particle of light; its C-parity is negative. P-symmetry or in other words - spatial parity - the mirror symmetry between lefthanded and right-handed objects and its violation was known to people long before the first scientific papers on the concepts of left and right appeared. In the XIXth century the importance of left-right asymmetry (dissymmetry) for the processes of life became evident due to Luis Pasteur and others. With the advent of quantum mechanics in the 1920s it was decided that biological dissymmetry is based on the P-symmetry of the basic fundamental interactions. In the language using the concepts of Hamiltonian or Lagrangian that meant that scalar and pseudo-scalar terms in them cannot coexist. For almost 30 years it was believed that only scalar terms are present and no pseudoscalar terms could be added. This ended with the discovery of the discovery of the V-A weak current. T-symmetry as well as P-symmetry was mainly formulated in the framework of quantum mechanics around 1930 by Eugene Wigner. In the language using the concepts of Hamiltonian or Lagrangian the time-reversal invariance meant that the coupling constants of scalar and pseudocalar terms must be real.

\subsection{Electric Dipole Moments (EDM) of Protons and Deuterons}

EDM are one of the keys to understand the origin of our Universe [Sakharov, 1967]. Andrei Sakharov formulated three conditions for baryogenesis:

1. Early in the evolution of the universe, the baryon number conservation must be violated sufficiently strongly,

2. The $\mathrm{C}$ and $\mathrm{CP}$ invariances, and $\mathrm{T}$ invariance thereof, must be violated, and

3. At the moment when the baryon number is generated, the evolution of the universe must be out of thermal equilibrium.

CP violation in kaon decays is known since 1964, it has been observed in B-decays and charmed meson decays. The Standard Model (SM) accommodates CP violation via the phase in the Cabibbo-Kobayashi-Maskawa matrix.

$\mathrm{CP}$ and $\mathrm{P}$ violation entail nonvanishing $\mathrm{P}$ and $\mathrm{T}$ violating electric dipole moments (EDM) of elementary particles $\vec{d}=d \vec{s}$. Although extremely successful in many aspects, the SM has at least two weaknesses: neutrino oscillations do require extensions of the SM and, most importantly, the SM mechanisms fail miserably in the expected baryogenesis rate.

Simultaneously, the SM predicts an exceedingly small electric dipole moment of nucleons $10^{-33}<d_{n}<10^{-31} e \cdot \mathrm{cm}$, way below the current upper bound for the neutron EDM, $d_{n}<2.9 \times$ $10^{-26} e \cdot \mathrm{cm}$. In the quest for physics beyond the SM one could follow either the high energy trail or look into new methods which offer very high precision and sensitivity. Supersymmetry is one of the most attractive extensions of the SM and 
S. Weinberg emphasized [Weinberg, 1993]: "Endemic in supersymmetric (SUSY) theories are CP violations that go beyond the SM. For this reason it may be that the next exciting thing to come along will be the discovery of a neutron electric dipole moment."

The SUSY predictions span typically $10^{-29}<d_{n}<10^{-24} \mathrm{e} \cdot \mathrm{cm}$ and precisely this range is targeted in the new generation of EDM searches [Roberts, Marciano, 2010]. There is consensus among theorists that measuring the EDM of the proton, deuteron and helion is as important as that of the neutron. Furthermore, it has been argued that T-violating nuclear forces could substantially enhance nuclear EDM [Flambaum, Khriplovich, Sushkov, 1986]. At the moment, there are no significant direct upper bounds available on $d_{p}$ or $d_{d}$. Non-vanishing EDMs give rise to the precession of the spin of a particle in an electric field. In the rest frame of a particle

$$
\dot{s}_{n}=\varepsilon_{n m k}\left(\Omega_{m} s_{k}+d_{m} E_{k}\right), \Omega_{m}=-\mu B_{m},
$$

where in terms of the lab frame fields

$$
\begin{aligned}
& B_{n}=\gamma\left(B_{n}^{l}-\varepsilon_{n m k} \beta_{m} E_{k}^{l}\right) \\
& E_{n}=\gamma\left(E_{n}^{l}+\varepsilon_{n m k} \beta_{m} B_{k}^{l}\right)
\end{aligned}
$$

Now we can apply the Hamiltonization and optimal control theory methods to this dynamical system.

\subsection{Relativistic Heavy Ion Collider (RHIC)}

RHIC is one of only two operating heavy-ion colliders, and the only spin-polarized proton collider ever built. Located at Brookhaven National Laboratory (BNL) in Upton, New York, and used by an international team of researchers, it is the only operating particle collider in the US. By using RHIC to collide ions traveling at relativistic speeds, physicists study the primordial form of matter that existed in the universe shortly after the Big Bang. By colliding spin-polarized protons, the spin structure of the proton is explored.

RHIC is now the second-highest-energy heavy-ion collider in the world. As of November 7, 2010, the LHC has collided heavy ions of lead at higher energies than RHIC. The LHC operating time for ions is limited to about one month per year.

In 2010, RHIC physicists published results of temperature measurements from earlier experiments which concluded that temperatures in excess of 4 trillion kelvin had been achieved in gold ion collisions, and that these collision temperatures resulted in the breakdown of "normal matter" and the creation of a liquid-like quark-gluon plasma.

RHIC is an intersecting storage ring particle accelerator. Two independent rings (arbitrarily denoted as "Blue" and "Yellow" rings, see also the photograph) circulate heavy ions and/or protons in opposite directions and allow a virtually free choice of colliding positively charged particles (the eRHIC upgrade will allow collisions between positively and negatively charged particles). The RHIC double storage ring is itself hexagonally shaped and 3,834 m long in circumference, with curved edges in which stored particles are deflected and focused by 1,740 superconducting niobium-titanium magnets. The dipole magnets operate at $3.45 \mathrm{~T}$. The six interaction points (between the particles circulating in the two rings) are at the middle of the six relatively straight sections, where the two rings cross, allowing the particles to collide. The interaction points are 
enumerated by clock positions, with the injection near 6 o'clock. Two large experiments, STAR(RHIC :) and PHENIX, are located at 6 and 8 o'clock respectively.

\subsection{Cumulative particle production (CPP)}

CPP is a process, when a heavy projectile produce lighter particle with higher velocity. Let us consider a heavy particle with momentum mass $M$, velocity $v$, colliding with a fixed target and producing a lighter particle with mass $m$ and velocity $V$. In the medium we have not the momentum conservation and generally we have $M v>m V$. When $V>v$, we say that we have CPP, because if we imaging the initial particle as a bound state of weakly coupled particles of mass $m$, the velocity $V$ can not be more than $v$. In CPP we have a collective effect in which constituents somehow transfer their momentum to the produced particle.

\subsection{Cosmic rays}

Cosmic rays are very high-energy particles, mainly originating outside the Solar System. They may produce showers of secondary particles that penetrate and impact Earth's atmosphere and sometimes even surface. Comprised primarily of high-energy protons and atomic nuclei, their origin has, until recently, been a mystery. With data from the Fermi space telescope published in February 2013, Ackermann et al, 2013] it is now known that cosmic rays primarily originate from the supernovae of massive stars, with each explosion producing roughly $3 \times 10^{42}-3 \times 10^{43} J$ of cosmic rays.

The term "cosmic rays" was coined in the 1920s by Robert Millikan who made measurements of ionization due to cosmic rays from deep under water to high altitudes and around the globe. During the years from 1930 to 1945, a wide variety of investigations confirmed that the primary cosmic rays are mostly protons, and the secondary radiation produced in the atmosphere is primarily electrons, photons and muons. In 1948, observations with nuclear emulsions carried by balloons to near the top of the atmosphere showed that approximately $10 \%$ of the primaries are helium nuclei (alpha particles) and $1 \%$ are heavier nuclei of the elements such as carbon, iron, and lead.

Of primary cosmic rays, which originate outside of Earth's atmosphere, about $99 \%$ are the nuclei (stripped of their electron shells) of well-known atoms, and about $1 \%$ are solitary electrons (similar to beta particles). Of the nuclei, about $90 \%$ are simple protons, i. e. hydrogen nuclei; $9 \%$ are alpha particles, and $1 \%$ are the nuclei of heavier elements. A very small fraction are stable particles of antimatter, such as positrons or antiprotons, and the precise nature of this remaining fraction is an area of active research.

Cosmic rays attract great interest practically, due to the damage they inflict on microelectronics and life outside the protection of an atmosphere and magnetic field, and scientifically, because the energies of the most energetic ultra-high-energy cosmic rays (UHECRs) have been observed to approach $3 \times 10^{20} \mathrm{eV}$, about 40 million times the energy of particles accelerated by the Large Hadron Collider (LHC). At 50J, the highest-energy ultra-high-energy cosmic rays have energies comparable to the kinetic energy of a 90-kilometre-per-hour (56 mph) baseball.

As a result of these discoveries, there has been interest in investigating cosmic rays of even greater energies. Most cosmic rays, however, do not have such extreme energies; the energy distribution of cosmic rays peaks at $0.3 \mathrm{GeV}\left(4.8 \times 10^{-11} \mathrm{~J}\right)$. 
There is a theoretical Greisen-Zatsepin-Kuzmin limit to the energies of cosmic rays from long distances (about 160 million light years) which occurs above $10^{20} \mathrm{eV}$ because of interactions with the remnant photons from the big bang origin of the universe. The highest energy events are strongly correlated with the locations of active galactic nuclei (AGNs), where bare protons are believed to be accelerated by strong magnetic fields associated with the large black holes at the AGN centers to energies of $10^{20} \mathrm{eV}$ and higher.

Most galactic cosmic rays are probably accelerated in the blast waves of supernova remnants. This doesn't mean that the supernova explosion itself gets the particles up to these speeds. The remnants of the explosions, expanding clouds of gas and magnetic field, can last for thousands of years, and this is where cosmic rays are accelerated. Bouncing back and forth in the magnetic field of the remnant randomly lets some of the particles gain energy, and become cosmic rays. Eventually they build up enough speed that the remnant can no longer contain them, and they escape into the Galaxy. Because the cosmic rays eventually escape the supernova remnant, they can only be accelerated up to a certain maximum energy, which depends upon the size of the acceleration region and the magnetic field strength. However, cosmic rays have been observed at much higher energies than supernova remnants can generate, and where these ultra-high-energies come from is a big question. Perhaps they come from outside the Galaxy, from active galactic nuclei, quasars or gamma ray bursts. Or perhaps they're the signature of some exotic new physics: superstrings, exotic dark matter, strongly-interacting neutrinos, or topological defects in the very structure of the universe. Questions like these tie cosmic-ray astrophysics to basic particle physics and the fundamental nature of the universe.

Cosmic rays originate as primary cosmic rays, which are those originally produced in various astrophysical processes. Primary cosmic rays are composed primarily of protons and alpha particles (99\%), with a small amount of heavier nuclei ( $1 \%$ ) and an extremely minute proportion of positrons and antiprotons. Secondary cosmic rays, caused by a decay of primary cosmic rays as they impact an atmosphere, include neutrons, pions, positrons, and muons. Of these four, the latter three were first detected in cosmic rays.

\subsection{Spherical model of the multiparticle production}

Now we would like to consider a model of multiparticle production based on the d-dimensional sphere, and (try to) motivate the values of the NBD parameter $k$. The volum of the d-dimensional sphere with radius $r$, in units of hadron size $r_{h}$ is

$$
v(d, r)=\frac{\pi^{d / 2}}{\Gamma(d / 2+1)}\left(\frac{r}{r_{h}}\right)^{d}
$$

Note that,

$$
\begin{aligned}
& v(0, r)=1, v(1, r)=2 \frac{r}{r_{h}}, \\
& v(-1, r)=\frac{1}{\pi} \frac{r_{h}}{r}
\end{aligned}
$$

If we identify this dimensionless quantity with corresponding coulomb energy formula,

$$
\frac{1}{\pi}=\frac{e^{2}}{4 \pi}
$$


we find $e= \pm 2$.

For less then -1 even integer values of $d$, and $r \neq 0, v=0$. For negative odd integer $d=-2 n+1$

$$
\begin{aligned}
& v(-2 n+1, r)=\frac{\pi^{-n+1 / 2}}{\Gamma(-n+3 / 2)}\left(\frac{r_{h}}{r}\right)^{2 n-1}, n \geq 1, \\
& v(-3, r)=-\frac{1}{2 \pi^{2}}\left(\frac{r_{h}}{r}\right)^{3}, v(-5, r)=\frac{3}{4 \pi^{3}}\left(\frac{r_{h}}{r}\right)^{5}
\end{aligned}
$$

Note that,

$$
v(2, r) v(3, r) v(-5, r)=\frac{1}{\pi}, v(1, r) v(2, r) v(-3, r)=-\frac{1}{\pi}
$$

Using ([0.12), for higher values of the space dimension, for the volume of the sphere, we obtain

$$
V(d, r)=\frac{\pi^{\frac{d}{2}}}{\Gamma\left(\frac{d}{2}+1\right)}\left(\frac{r}{r_{h}}\right)^{d}=\frac{\pi^{\frac{d}{2}} e^{\frac{d}{2}}}{\left(\frac{d}{2}\right)^{\frac{d}{2}}}\left(1+O\left(\frac{1}{d}\right)\right)\left(\frac{r}{r_{h}}\right)^{d}
$$

To the following evolution of the dimension with $r$ corresponds invariant volume of the sphere

$$
d(r)=2 \pi e\left(\frac{r}{r_{h}}\right)^{2}\left(1+O\left(\frac{1}{r^{2}}\right)\right)
$$

We postulate that after collision, it appear intermediate state with almost spherical form and constant energy density. Than the radius of the sphere rise, dimension decrease, volume remains constant. At the last moment of the expansion, when the crossection of the one dimensional sphere - string become of order of hadron size, hadronic string divide in $k$ independent sectors which start to radiate hadrons with geometric (Boze-Einstein) distribution, so all of the string final state radiate according to the NBD distribution.

So, from the volume of the hadronic string,

$$
v=\pi\left(\frac{r}{r_{h}}\right)^{2} \frac{l}{r_{h}}=\pi k
$$

we find the NBD parameter $k$,

$$
k=\frac{\pi^{d / 2-1}}{\Gamma(d / 2+1)}\left(\frac{r}{r_{h}}\right)^{d}
$$

Knowing, from experimental date, the parameter $k$, we can restrict the region of the values of the parameters $d$ and $r$ of the primordial sphere (PS),

$$
\begin{aligned}
& r(d)=\left(\frac{\Gamma(d / 2+1)}{\pi^{d / 2}-1} k\right)^{1 / d} r_{h}, \\
& r(3)=\left(\frac{3}{4} k\right)^{1 / 3} r_{h}, r(2)=k^{1 / 2} r_{h}, r(1)=\frac{\pi}{2} k r_{h}
\end{aligned}
$$

If the value of $r(d)$ will be a few $r_{h}$, the matter in the PS will be in the hadronic phase. If the value of $r$ will be of order $10 r_{h}$, we can speak about deconfined, quark-gluon, Glukvar, phase. From the 
formula ( $(25.88)$, we see, that to have for the $r$, the value of order $10 r_{h}$, in $d=3$ dimension, we need the value for $k$ of order 1000, which is not realistic.

So in our model, we need to consider the lower than one, fractal, dimensions. It is consistent with the following intuitive picture. Confined matter have point-like geometry, with dimension zero. Primordial sphere of Glukvar have nonzero fractal dimension, which is less than one,

$$
\begin{aligned}
& k=3, r(0.7395) / r_{h}=10.00, \\
& k=4, r(0.8384) / r_{h}=10.00
\end{aligned}
$$

From the experimental data we find the parameter $k$ of the NBD as a function of energy, $k=k(s)$. Then, by our spherical model, we construct fractal dimension of the Glukvar as a function of $k(s)$.

If we suppose that radius of the primordial sphere $r$ is of order (or less) of $r_{h}$. Than we will have higher dimensional PS, e.g.

\begin{tabular}{|c|c|c|}
\hline $\mathrm{d}$ & $r / r_{h}$ & $\mathrm{k}$ \\
\hline 3 & 1.3104 & 3.0002 \\
4 & 1.1756 & 3.0003 \\
6 & 1.1053 & 2.9994 \\
8 & 1.1517 & 3.9990 \\
\hline
\end{tabular}

\subsection{Extra dimension effects at high energy and large scale Universe}

With extra dimensions gravitation interactions may become strong at the LHC energies,

$$
V(r)=\frac{m_{1} m_{2}}{m^{2+d}} \frac{1}{r^{1+d}}
$$

If the extra dimensions are compactified with(in) size $\mathrm{R}$, at $r>>R$,

$$
V(r) \simeq \frac{m_{1} m_{2}}{m^{2}(m R)^{d}} \frac{1}{r}=\frac{m_{1} m_{2}}{M_{P l}^{2}} \frac{1}{r},
$$

where (4-dimensional) Planck mass is given by

$$
M_{P l}^{2}=m^{2+d} R^{d},
$$

so the scale of extra dimensions is given as

$$
R=\frac{1}{m}\left(\frac{M_{P l}}{m}\right)^{\frac{2}{d}}
$$

If we take $m=1 T e V,\left(\mathrm{GeV}^{-1}=0.2 \mathrm{fm}\right)$

$$
\begin{aligned}
& R(d)=2 \cdot 10^{-17}\left(\frac{M_{P l}}{1 T e V}\right)^{\frac{2}{d}} \mathrm{~cm}, \\
& R(1)=2 \cdot 10^{15} \mathrm{~cm}, \\
& R(2)=0.2 \mathrm{~cm} ! \\
& R(3)=10^{-7} \mathrm{~cm} ! \\
& R(4)=2 \cdot 10^{-9} \mathrm{~cm}, \\
& R(6) \sim 10^{-11} \mathrm{~cm}
\end{aligned}
$$


Note that lab measurements of $G_{N}\left(=1 / M_{P l}^{2}, M_{P l}=1.2 \times 10^{19} \mathrm{GeV}\right)$ have been made only on scales of about $1 \mathrm{~cm}$ to $1 \mathrm{~m} ; 1$ astronomical unit(AU) (mean distance between Sun and Earth) is $1.5 \times$ $10^{13} \mathrm{~cm}$; the scale of the periodic structure of the Universe, $L=128 \mathrm{Mps} \simeq 4 \cdot 10^{26} \mathrm{~cm}$. It is curious which (small) value of the extra dimension corresponds to $L$ ?

$$
\begin{aligned}
d & =2 \frac{\ln \frac{M_{P l}}{m}}{\ln (m L)}=0.74, m=1 T e V, \\
& =0.81, m=100 \mathrm{GeV}, \\
& =0.07, m=10^{17} \mathrm{GeV} .
\end{aligned}
$$

\subsection{Large-scale structure of the Universe}

In 1929 astronomer Hubble discovered expansion of the Universe predicted early by mathematician Freedman in 1922. The expansion of the Universe means that the distance between any two points of it, $r(t)$ rise with the time according to the Hubble's low

$$
\dot{r}=H(t) r, r(t)=r_{0} \exp \left(\int_{t_{0}}^{t} d \tau H(\tau)\right)
$$

where $H(t)$ is time dependent (running) Hubble's constant. Today

$$
H=100 \mathrm{~h}=70.5 \mathrm{~km} / \mathrm{s} / \mathrm{Mpc}, 1 \mathrm{Mpc}=10^{6} \mathrm{pc}, \mathrm{pc} \simeq 3.26 \mathrm{lyrs}
$$

Large-scale structures of the Universe (LSSU) are considered in the region from $1 M p c$ to several hundred $M p c$. On these scales galactics can be considered as point particles in good approximation. In the co-moving system $r=a(t) x$, the coordinate $x$ and velocity $v$ of a galactic is defined from the Hubble low

$$
v=\dot{r}-H r=a(t) \dot{x}, r(t)=a(t) x, \dot{a}=H a, H=\frac{d \ln a}{d t} .
$$

The scale factor $a(t)$ is connected with red shift of spectrum $z$ as

$$
a(t)=\frac{1}{1+z(t)}, a\left(t_{0}\right)=1 \Leftrightarrow z\left(t_{0}\right)=0
$$

The scale factor $a(t)$ monotonically rise with time and describes homogeneous expansion.

\subsection{Sloan Digital Sky Survey}

The survey makes the data releases available over the Internet. The SkyServer provides a range of interfaces to an underlying Microsoft SQL Server. Both spectra and images are available in this way, and interfaces are made very easy to use so that, for example, a full color image of any region of the sky covered by an SDSS data release can be obtained just by providing the coordinates. The data are available for non-commercial use only, without written permission. The SkyServer also provides a range of tutorials aimed at everyone from schoolchildren up to professional astronomers. 


\subsection{Spatial Periodicity of Galaxy Number Counts}

It is currently believed that the expansion of our universe at present is in an accelerated stage. The measurements of the temperature anisotropy of the cosmic microwave background radiation (hereafter $\mathrm{CMB}$ ) by the Wilkinson microwave anisotropy probe (WMAP) have indicated that our universe is flat. The high precision of these and other observations have enabled us to intercompare and verify various candidate cosmological models.

A flat $\Lambda C D M$ model consisting of Einstein's cosmological constant $\Lambda$ and cold dark matter, in addition to the ordinary baryonic matter and radiation is currently the standard model because it can account reasonably well for the acceleration of the cosmic expansion the spatial power spectrum of the CMB temperature anisotropy observed by WMAP satellite, and the primordial abundance of the light chemical elements produced through big-bang nucleosynthesis. Nevertheless, some key questions remain as to the validity of the standard $\Lambda C D M$ model. For instance, the physical origin of the cosmological constant is presently unknown, although it might possibly be a component of vacuum energy. As such, we have yet to understand why the energy density of the cosmological constant remains unchanged with time. The numerical size of the $\Lambda$-term estimated based on a particle physics consideration is generally more than 120 orders of magnitude too large to be compared to the observed values.

As an alternative approach to circumvent these problems of the $\Lambda$-term, cosmological models possessing quintessence or a phantom have been proposed. These methods interpret the dark energy in terms of the type of time-varying scalar field.

The second consideration is that the cosmic age of the standard model, which is largely based on the WMAP 3-yr data, is supposed to be 13.7 Gyrs, whereas the possibility of it being, for instance, approximately 14-15 Gyrs has been raised based on observations of certain globular clusters.

\subsection{Fermi- and bose-statistics and Cosmological constant problem}

One of the stranger consequences of quantum mechanics is that even empty space has energy. The problem of how to calculate this vacuum energy is arguably the most intriguing mystery in theoretical physics. For decades physicists tried to understand why this energy is so small, but no definitive solution has yet been found. Vacuum is empty space, but it is far from being "nothing". It is a complicated physical object in which particles like electrons, positrons and photons are being incessantly produced and destroyed by quantum fluctuations. Such virtual particles exist only for a fleeting moment, but their energies combine to endow each cubic centimeter of space with a nonzero energy. This vacuum energy density does not change in time; it is called the cosmological constant and is usually denoted by $\Lambda$. The trouble is that theoretical calculations of $\Lambda$ give ridiculously large numbers, 120 orders of magnitude greater than what is observed. According to Einstein's General Relativity, vacuum energy produces a repulsive gravitational force, and if the energy were so large, its gravity would have instantly blown the universe apart. It is conceivable that positive vacuum energy contributions from some particle species are compensated by negative contributions from other species, so that the net result is close to zero. But then the compensation must be amazingly precise, up to 120 decimal places. There seems to be no good reason for such a miraculous cancellation. Until recently, the great majority of physicists believed that something so 
small could only be zero: some hidden symmetry should force exact cancellation of all contributions to the cosmological constant. However, observations of distant supernova explosions in the late 1990's yielded the surprising discovery that the expansion of the universe accelerates with time [Riess et al, 1998], [Perlmutter et al, 1999]- a telltale sign of cosmic repulsion caused by a nonzero (positive) cosmological constant.

The observed magnitude of $\Lambda$ has brought about another mystery: its value is roughly twice the average energy density (or, equivalently, mass density) of matter in the universe. This is surprising because the matter and vacuum densities behave very differently with cosmic expansion. The vacuum density remains constant, while the matter density decreases; it was much greater in the past and will be much smaller in the future. Why, then, do we happen to live at the very special epoch when the two densities are so close to one another? This became known as the cosmic coincidence problem.

WMAP's measurements played the key role in establishing the current Standard Model of Cosmology: the Lambda-CDM model. WMAP data are very well fit by a universe that is dominated by dark energy in the form of a cosmological constant. Other cosmological data are also consistent, and together tightly constrain the Model. In the Lambda-CDM model of the universe, the age of the universe is $13.772 \pm 0.059$ billion years. The WMAP mission's determination of the age of the universe to better than $1 \%$ precision was recognized by the Guinness Book of World Records. The current expansion rate of the universe (Hubble constant) is of

$$
69.32 \pm 0.80 \mathrm{~km} \cdot \mathrm{s}^{-1} \cdot \mathrm{Mpc}^{-1} .
$$

The content of the universe presently consists of $4.628 \pm 0.093 \%$ ordinary baryonic matter; $24.02+$ $0.88(-0.87) \%$ Cold dark matter (CDM) that neither emits nor absorbs light; and $71.35+0.95(-0.96) \%$ of dark energy in the form of a cosmological constant that accelerates the expansion of the universe. Less than $1 \%$ of the current contents of the universe is in neutrinos, but WMAP's measurements have found, for the first time in 2008, that the data prefers the existence of a cosmic neutrino background with an effective number of neutrino species of $3.26 \pm 0.35$. The WMAP measurements also support the cosmic inflation paradigm in several ways, including the flatness measurement, and Euclidean flat geometry, with curvature $\Omega_{k}=-0.0027+0.0039(-0.0038)$.

\subsection{Cosmological constant (CC)}

The polish priest Nicolaus Copernicus (1473-1543) started a revolution with his heliocentric cosmology that displaced the Earth from the center of the Universe. His remarkable shift in paradigm continues to this day, the cornerstone of a concept we now call the Cosmological Principle, in which the Universe is assumed to be homogeneous and isotropic, without a center or boundary. But few realize that even this high degree of symmetry is insufficient for cosmologists to build a practical model of the Universe from the equations of General Relativity. The missing ingredient emerged from the work of mathematician Hermann Weyl (1885-1955), who reasoned that on large scales the Universe must be expanding in an orderly fashion. He argued that all galaxies move away from each other. In this view, the evolution of the universe is a time-ordered sequence of 3-dimensional space-like hypersurfaces, each of which satisfies the Cosmological Principle-an intuitive picture of regularity formally expressed as the Weyl postulate. Together, these two philosophical inputs allow us to use a special time coordinate, called the cosmic time $t$, to represent 
how much change has occurred since the big bang, irrespective of location. The Weyl postulate reduces formally to the mathematical expression $R(t)=a(t) r$, meaning that the proper distance $R(t)$ between any two spacetime points must be the product of a fixed, co-moving distance $r$, which never changes even as the Universe expands, and a universal function of time $a(t)$ independent of position, but not necessarily of time. A galaxy a proper distance $R(t)$ from us must therefore be receding at speed

$$
\dot{R}(t)=\dot{a} r=\frac{\dot{a}}{a} R=H(t) R
$$

- the Hubble Law. The Hubble constant $H(t)$ is independent of position. Notice how easily such a simple consequence of the Weyl postulate accounts for all of Hubble's famous observations, which convinced even Einstein-an early advocate of the static Universe-that the cosmos is expanding at a speed proportional to proper distance. There is much to learn about the Universe-perhaps even its beginnings-by understanding $H$ or $a(t)$, and so much of the effort in cosmological research is dedicated to this task.

In Cosmology, the cosmological constant $\mathrm{CC} \Lambda$ is equivalent to an energy density in otherwise empty space. It was proposed by Albert Einstein as a modification of his original theory of general relativity to achieve a stationary universe. It appears in Einstein's field equation in the form of

$$
R_{\mu v}+\left(\Lambda-\frac{1}{2} R\right) g_{\mu v}=a T_{\mu v}, a=\frac{8 \pi G}{c^{4}}
$$

$\mathrm{CC}$ has the same effect as an intrinsic energy density of the vacuum, $\rho_{v a c}$ (and an associated pressure). In this context it is commonly moved onto the right-hand side of the equation,

$$
R_{\mu v}-\frac{1}{2} R_{\mu v}=a\left(T_{\mu v}-\rho_{v a c} g_{\mu v}\right), a=\frac{8 \pi G}{c^{4}}=8 \pi
$$

where

$$
\Lambda=\frac{8 \pi G}{c^{4}} \rho_{v a c}=8 \pi \rho_{v a c},
$$

and the unit conventions of general relativity are used $(\mathrm{G}=\mathrm{c}=1)$. It is common to quote values of energy density directly, though still using the name "cosmological constant". The Lambda-CDM model of the Universe (the most accepted modern cosmological model) asserts that $\Lambda$ is positive, although negligible even by the scale of Milky Way. A positive vacuum energy density resulting from a cosmological constant implies a negative pressure, and vice versa. If the energy density is positive, the associated negative pressure will drive an accelerated expansion of the universe, as observed. CC is a subject of theoretical and empirical interest. Empirically, the onslaught of cosmological data in the past decades strongly suggests that our universe has a positive cosmological constant. The explanation of this small but positive value is an outstanding theoretical challenge. The current standard model of cosmology, the Lambda-CDM model, includes the cosmological constant, which is measured to be on the order of $10^{-52} \mathrm{~m}^{-2}$, in metric units. Multiplied by other constants that appear in the equations, it is often expressed as $10^{-35} \mathrm{~s}^{-2}, 10^{-47} \mathrm{GeV}^{4}, 10^{-29} \mathrm{~g} / \mathrm{cm}^{3}$ [Tegmark, 2004]. In terms of Planck units, and as a natural dimensionless value $\mathrm{CC}$ is on the order of $10^{-122}$ [Barrow, Shaw, 201]]. A positive cosmological constant has surprising consequences, 
such as a finite maximum entropy of the observable universe [Dyson, Kleban, Susskind, 2002]. A problem arises with inclusion of the cosmological constant in the standard model: i.e., the appearance of solutions with regions of discontinuities. Discontinuity also affects the past sign of the pressure of the cosmological constant, changing from the current negative pressure to attractive, with lookback towards the early Universe. The cosmological time, $d t$, diverges for any finite interval, $d s$, associated with an observer approaching the cosmological horizon, representing a physical limit to observation for the standard model when the cosmological term is included. This is a key requirement for a complete interpretation of astronomical observations, particularly pertaining to the nature of dark energy and the cosmological constant. All of these findings should be considered major shortcomings of the standard model, but only when the cosmological constant term is included.

A major outstanding problem is that most quantum field theories predict a huge value for the quantum vacuum. A common assumption is that the quantum vacuum is equivalent to the cosmological constant. Although no theory exists that supports this assumption, arguments can be made in its favor. Such arguments are usually based on dimensional analysis and effective field theory. If the universe is described by an effective local quantum field theory down to the Planck scale, then we would expect a cosmological constant of the order of $m_{P l}^{4}$. The measured cosmological constant is smaller than this by a factor of $10^{-120}$. For TeV scale we will have instead the factor $10^{-56}$. This discrepancy has been called "the worst theoretical prediction in the history of physics!". Some supersymmetric theories require a cosmological constant that is exactly zero, which further complicates things. This is the cosmological constant problem, the worst problem of fine-tuning in physics: there is no known natural way to derive the tiny cosmological constant used in cosmology from particle physics.

One possible explanation for the small but non-zero value was noted by Steven Weinberg in 1987 following the anthropic principle [Weinberg, 1987]. Weinberg explains that if the vacuum energy took different values in different domains of the universe, then observers would necessarily measure values similar to that which is observed: the formation of life-supporting structures would be suppressed in domains where the vacuum energy is much larger. Specifically, if the vacuum energy is negative and its absolute value is substantially larger than it appears to be in the observed universe (say, a factor of 10 larger), holding all other variables (e.g. matter density) constant, that would mean that the universe is closed; furthermore, its lifetime would be shorter than the age of our universe, possibly too short for the intelligent life to form. On the other hand, a universe with a large positive cosmological constant would expand too fast, preventing galaxy formation. According to Weinberg, domains where the vacuum energy are compatible with life would be comparatively rare. Using this argument, Weinberg predicted that the cosmological constant would have a value of less than a hundred times the currently accepted value. In 1995 Weinberg's argument was refined by Alexander Vilenkin to predict a value for the cosmological constant that was only ten times the matter density, i.e. about three times the current value since determined [Dilenkin, 1995].

Let us describe a mechanism of estimation of the CC [Makhaldiani, 2003]. We suppose that we have a supersymmetric spectrum of the matter at some (small) temperature. In the simplest case of one supersymmetric harmonic oscillator, we have one bose- and one fermi-oscillators. The 
energy spectrum of the bose-oscillator is

$$
E_{n}=\hbar \omega\left(n+\frac{1}{2}\right), n=0,1,2, \ldots
$$

Corresponding statistical sum is

$$
Z_{B}=\sum_{n \geq 0} e^{-a\left(n+\frac{1}{2}\right)}=\frac{e^{-\frac{a}{2}}}{1-e^{-a}}=\frac{1}{2 \sinh \frac{a}{2}}, a=\frac{\hbar \omega}{k T}
$$

For fermi-oscillator we have

$$
\begin{aligned}
& E_{n}=\hbar \omega\left(n-\frac{1}{2}\right), n=0,1 \\
& Z_{F}=\sum_{n=0}^{1} e^{-a\left(n-\frac{1}{2}\right)}=e^{\frac{a}{2}}+e^{-\frac{a}{2}}=2 \cosh \frac{a}{2}
\end{aligned}
$$

For super-oscillator system composed from one fermi- and one bose-oscillators,

$$
Z=Z_{B} Z_{F}=e^{-\beta F}=\operatorname{coth} \frac{a}{2}=1+2 e^{-\frac{a}{2}}+\ldots, a=\frac{\hbar \omega}{k T} \gg 1
$$

so, the contribution of the supersymmetric oscillator in the cosmological constant $\Lambda \sim \ln Z \sim e^{-\frac{a}{2}} \simeq$ 0 , and a suppersymmetry of the effective action on the cosmological scale may solve cosmological constant problem and explain the dark matter and energy problem.

Let us estimate the energy-masse of the corresponding state for the temperature of the relict radiation as follows

$$
\begin{aligned}
& 10^{-120}=e^{-120 \ln 10} \Rightarrow a=\frac{\hbar \omega}{k T}=240 \ln 10 \Rightarrow m=\hbar \omega \simeq 553 T \\
& 300 K \simeq \frac{1}{38.7} \mathrm{eV} \Rightarrow 3 K \simeq 2.58 \times 10^{-4} \mathrm{eV} \Rightarrow m=0.143 \mathrm{eV}
\end{aligned}
$$

\subsection{Axion candidates for dark matter}

Astrophysical considerations place the axion mass in a range $10^{-2}-10^{-6} \mathrm{eV}$, [Kuster et al, 2008].

\subsection{Massive neutrinos}

Neutrino may be the same with its antiparticle-the Majorana neutrino, or may be different with its antiparticle-the Dirac neutrino. In some extensions of the SM, Dirac neutrino with mass $m_{D}$ reduce to two Majorana neutrinos with masses $m_{M}$ and $m_{n}$ in relation $m_{n} m_{M} \simeq m_{D}^{2}$ - the seesaw relation. It is also natural for $M_{D}$ to be of the order of $m_{l}$ or $m_{q}$, the mass of a typical charged lepton or quark. Now, if $m_{N} \gg m_{l}$ or $m_{q}$, then $m_{n} \ll m_{l}$ or $m_{q}$. For light quark $u$ mass $m_{u} \sim 1 \mathrm{MeV}$ so when $m_{M} \sim 10^{7} m_{u} \sim 10 \mathrm{TeV}, m_{n} \sim 10^{-7} m_{u} \sim 0.1 \mathrm{eV}$.

\subsection{Hadrons in a hot and dense medium}

Understanding the modification of hadron properties in a hot and dense medium is one of the most important issues, since it is deeply related to the restoration of chiral symmetry of quantum chromodynamics (QCD) [Brown and Rho, 1996]. The nucleon in medium also undergoes modification and eventually its structure gets altered. 


\subsection{Quark-Gluon Phase of QCD: Gluquar}

Most ordinary hadrons can only exist up to temperatures of about $150-170 \mathrm{MeV}$. Beyond that, chiral symmetry is restored and confinement is lost. We know that this hot state of matter - the QuarkGluon Plasma (QGP), Gluquar - existed in the early universe: the transition from the Gluquar to the hadronic world is the latest cosmological transition. The Gluquar can be re-created in accelerators.

\subsection{Valence Quark Counting}

In the development of hadron elliptic flow at RHIC it appears that elliptic flow of any hadron species depends on the transverse kinetic energy $K=m_{T}-m_{0}$ in a similar manner provided both $v_{2}$ and $K$ are divided by the number of constituent quarks, $n_{q}$, in a given hadron, $n_{q}=2$ for a meson and $n_{q}=3$ for a baryon. So, we have universal, scaling, form of distribution from which we obtain elliptic flow for concrete reaction by multiply $v_{2}$ and $K$ on corresponding valence quarks number, we have the Valence Quark Counting (VQC). Experimental studies indicate that the scaling holds up only until $K / n_{q} \simeq 1 \mathrm{GeV}$, Adare et al, 2007].

\subsection{Classically Scale Invariant Standard Model}

The discovery of the $125 \mathrm{GeV}$ scalar particle [Aad et al. ATLAS Collaboration, 2012], [Chatrchyan et al. CMS Collaboration, 2012] with the properties of the Standard Model Higgs boson together with the so far negative results for searches of supersymmetry are pointing to a possibility of a very different model building paradigm based on a minimally extended Standard Model with manifest classical scale invariance.

In the minimal classically scale-invariant extension of the Standard Model the Higgs mechanism is triggered and the electroweak symmetry breaking is generated radiatively by the ColemanWeinberg sector which is coupled to the SM Higgs [Khoze, 2013]. Higgs portal interactions of the theory include an additional singlet which is also non-minimally coupled to gravity. The singlet field responsible for inflation also gives a viable dark matter candidate.

\subsection{Abraham-Lorentz-Dirac equation for the charged particle dynamics}

Dirac relativistic theory of classical point electron [Dirac, 1938] has its equation of motion

$$
\frac{d p_{\mu}}{d \tau}=F_{\mu}+f_{\mu}
$$

where $p_{\mu}$ is the particle's 4-momentum, $\tau$ is the particle's proper time, and $f_{\mu}$ is the covariant generalization of the radiative reaction force

$$
f=\frac{2 e^{2}}{3 c^{3}} \ddot{v}
$$

as

$$
\begin{aligned}
& f_{\mu}=k\left(\frac{d^{2} p_{\mu}}{d \tau^{2}}+\frac{p_{\mu}}{m c^{2}} \frac{d p_{v}}{d \tau} \frac{d p^{v}}{d \tau}\right) \\
& =k\left(\ddot{p_{\mu}}+\frac{\dot{p}^{2}}{m c^{2}} p_{\mu}\right), k=\frac{2 e^{2}}{3 c^{3}}
\end{aligned}
$$


Note that, $p_{\mu} f^{\mu}=0$. In the case of the one dimensional space, the motion equation reduce to [Џackson, 1999]

$$
\dot{p}=k\left(\ddot{p}-\frac{p \dot{p}^{2}}{p^{2}+m^{2} c^{2}}\right)+f(\tau) \sqrt{1+\frac{p^{2}}{m^{2} c^{2}}},
$$

where $p$ is the momentum in the direction of motion and $f(\tau)$ is the ordinary Newtonian force as a function of the proper time. After substitution $p=m c \sinh y$, this equation reduce to the AbrahamLorentz equation [Dackson, 1999]

$$
\dot{y}=f+k \ddot{y}
$$

with following general solution

$$
\begin{aligned}
& y(\tau)=y_{0}(\tau)+\int G(\tau, t) f(t) d t, \\
& \left(\partial_{\tau}-k \partial_{\tau \tau}\right) G(\tau, t)=\delta(\tau-t), \\
& \left(\partial_{\tau}-k \partial_{\tau \tau}\right) y_{0}(\tau)=0 .
\end{aligned}
$$

\subsection{Electrodynamics with external currents}

In a good approximation the Maxwell electrodynamics can be approximated as

$$
\begin{aligned}
& \partial^{\mu} F_{\mu v}=J_{\mu}, \\
& \partial^{\mu} \tilde{F}_{\mu v}=0, \\
& \tilde{F}_{\mu v}=\frac{1}{2} \varepsilon_{\mu v \rho \sigma} F^{\rho \sigma},
\end{aligned}
$$

where $J_{\mu}$ is external current- a given function of the spacetime. From the antisymmetric property of $F_{\mu \nu}$, we obtine the conservation of the current(charge),

$$
\partial^{\mu} J_{\mu}=0
$$

Introducing the vector potential $A_{\mu}$ and the following ansatz

$$
F_{\mu v}=\partial_{\mu} A_{v}-\partial_{v} A_{\mu}
$$

the second equation of the Maxwell equations is solved automatically. The first equation become

$$
\begin{aligned}
& \square A_{v}-\partial_{v}(\partial A)=J_{v}, \\
& \square=\partial^{\mu} \partial_{\mu}, \partial A=\partial^{\mu} A_{\mu} .
\end{aligned}
$$

With the gauge condition $\partial A=$ const , the solution is

$$
\begin{aligned}
& A_{\mu}=a_{\mu}+\square^{-1} J_{\mu}, \\
& \square a_{\mu}=0 .
\end{aligned}
$$

Without any gauge condition,

$$
A_{\mu}=a_{\mu}+G_{\mu}^{v} J_{v}
$$




$$
\begin{aligned}
& G_{\mu}^{v} J_{v}(x)=\int G_{\mu}^{v}(x-y) J_{v}(y) d y, \\
& G_{\mu v}(x)=\int \frac{d x^{D}}{(2 \pi)^{D}} G_{\mu v}(k) e^{i k x}, \\
& G_{\mu v}(k)=\frac{1}{k^{2}} P_{\mu v}, P_{\mu v}=\left(g_{\mu v}-\frac{k_{\mu} k_{v}}{k^{2}}\right), P^{2}=P, P a=0,
\end{aligned}
$$

where under $k^{2}$, we understand $k^{2}+i 0$

If we consider point charge

$$
\begin{aligned}
& J_{0}(x)=e \delta(x-x(t)), \delta(x-x(t))=\prod_{n=1}^{D-1} \delta\left(x_{n}-x_{n}(t)\right), \int d^{D-1} x J_{0}=e ; \\
& J_{n}=e v_{n} \delta(x-x(t)), \\
& \partial_{t} J_{0}(x)=-e \dot{x_{n}} \partial_{n} \delta ; \partial_{n} J_{n}=e v_{n} \partial_{n} \delta+e\left(\partial_{n} V_{n}\right) \delta(x-x(t)),
\end{aligned}
$$

due to the current conservation, we have

$$
\dot{x_{n}}=v_{n}, \partial_{n} v_{n}=0
$$

In particular, if the velocity dos not changes direction with changing coordinates, it must be constant and there is the rest frame of the point particle.

\subsection{Riemann surfaces}

Any Riemann surface of genus $g=1,2$ can be written as a hyperelliptic curve, i.e they admit a map $f: \Sigma_{g} \rightarrow \mathbb{C} P^{1}$ to the Riemann sphere [Gritfiths and Harris, 1994]. For genus $g=1$ one has

$$
y^{2}=\prod_{n=1}^{4}\left(x-a_{n}\right), g=1
$$

and for genus $\mathrm{g}=2$ the curve is

$$
y^{2}=\prod_{n=1}^{6}\left(x-a_{n}\right), g=2
$$

where $\left\{a_{n} \in \mathbb{C}\right\}$ are branch points.

\subsection{Hyper-elliptic curves}

Hyper-elliptic curve is defined by

$$
y^{2}=P_{N}(x)
$$

where $P_{N}(x)$ is polynomial of degree $\mathrm{N}$. There are $2 \mathrm{~g}$ cycles $A_{i}, B_{i}, i=1 \ldots g$ which can be chosen as follows $\left(A_{i}, A_{j}\right)=0,\left(B_{i}, B_{j}\right)=0,\left(A_{i}, B_{j}\right)=d_{i j}$. For genus g hyper-elliptic curve there are exactly $\mathrm{g}$ holomorphic abelian-differentials of the first kind $\omega_{k}$ :

$$
\oint_{A_{i}} \omega_{k}=\delta_{i k}
$$


which are linear combinations of

$$
\frac{d x}{y}, \ldots, \frac{x^{(N-1) / 2} d x}{y}
$$

Period matrix is given by:

$$
\oint_{B_{i}} \omega_{k}=\tau_{i k}
$$

\subsection{Computation of the Determinant of a Generalized Vandermonde Matrix}

"Vandermonde" matrix is a matrix whose $(i, j)$-th entry is in the form of $x_{i}^{j}$. The matrix has a lot of applications in many fields such as signal processing and polynomial interpolations. We consider generalization of the matrix, when its $(\mathrm{i}, \mathrm{j})$ entry is $f_{j}\left(x_{i}\right)$ where $f_{j}(x)$ is a "fine" functions, e.g. polynomial of $x$ [Kitamoto, 2014].

\subsection{Constrained Dynamics}

Constrained dynamics represents a cornerstone of theoretical physics, as every relativistic theory and every theory with gauge symmetries necessarily possesses constraints. The classical Hamiltonian treatment of systems with constraints was developed by Dirac, [Dirac, 1950, 1964]. In the case of finite-dimensional systems his algorithm corresponds to rendering the equations of motion involutive.

\section{$25.64 p$-adic string theory}

p-adic string amplitudes can be obtained as tree amplitudes of the field theory with the following lagrangian and motion equation (see e.g. [Brekke, Freund, 1993])

$$
\begin{aligned}
& L=\frac{1}{2} \Phi Q_{p} \Phi-\frac{1}{p+1} \Phi^{p+1}, \\
& Q_{p} \Phi=\Phi^{p}, Q_{p}=p^{D} \\
& D=-\frac{1}{2} \triangle, \triangle=-\partial_{x_{0}}^{2}+\partial_{x_{1}}^{2}+\ldots+\partial_{x_{n-1}}^{2},
\end{aligned}
$$

$\Phi$ - is real scalar field on $D$-dimensional space-time with coordinates $x=\left(x_{0}, x_{1}, \ldots, x_{D-1}\right)$. We have trivial, $\Phi=0$ and $\Phi=1$, and following nontrivial solutions of the equation ([25.130)

$$
\Phi\left(x_{0}, x_{1}, \ldots, x_{D-1}\right)=p^{\frac{D}{2(p-1)}} e^{\frac{1-p^{-1}}{2 \ln p}\left(x_{0}^{2}-x_{1}^{2}-x_{2}^{2}-\ldots-x_{D-1}^{2}\right)}
$$

\subsection{5 p-adic solitons}

The equation ( 25.530 ) permits factorization of its solutions $\Phi(x)=\Phi\left(x_{0}\right) \Phi\left(x_{1}\right) \ldots \Phi\left(x_{D-1}\right)$, every factor of which fulfils one dimensional equation

$$
p^{\varepsilon \partial_{x}^{2}} \Phi(x)=\Phi(x)^{p}, \varepsilon= \pm \frac{1}{2}
$$

The trivial solution of the equations are $\Phi=0$ and $\Phi=1$. For nontrivial solution of ([25.133) , we have

$$
p^{\varepsilon \partial_{x}^{2}} \Phi(x)=e^{a \partial^{2}} \Phi(x)=\frac{1}{\sqrt{4 \pi a}} \int_{-\infty}^{\infty} d y e^{-\frac{1}{4 a} y^{2}+y \partial} \Phi(x)
$$




$$
=\frac{1}{\sqrt{4 \pi a}} \int_{-\infty}^{\infty} d y e^{-\frac{1}{4 a} y^{2}} \Phi(x+y)=\Phi(x)^{p}, a=\varepsilon \ln p
$$

If we (de quantize) put, $p=q$, and take (classical) limit, $q \rightarrow 1$, the motion equation reduce to

$$
\varepsilon \partial_{x}^{2} \Phi=\Phi \ln \Phi
$$

with solution

$$
\Phi(x)=e^{\frac{1}{2}} e^{\frac{x^{2}}{4 \varepsilon}}
$$

It is obvious that the anzac

$$
\Phi=A e^{b x^{2}}
$$

can pass the equation (25.534). Indeed, the solution is

$$
\begin{aligned}
& \Phi(x)=p^{\frac{1}{2(p-1)}} e^{\frac{1-p^{-1}}{4 \varepsilon \ln p} x^{2}} \\
& \Phi\left(x_{0}, x_{1}, \ldots, x_{D-1}\right)=p^{\frac{D}{2(p-1)}} e^{\frac{1-p^{-1}}{2 \ln p}\left(x_{0}^{2}-x_{1}^{2}-x_{2}^{2}-\ldots-x_{D-1}^{2}\right)}
\end{aligned}
$$

\subsection{Corresponding class of the motion equations}

Now, we can define the following class of motion equations

$$
Q_{q} F=F^{q},
$$

where

$$
Q_{q}=q^{D}, D=D_{1}\left(x_{1}\right)+\ldots+D_{l}\left(x_{l}\right),
$$

$D_{k}(x)$ is some (differential) operator depending on $x$. In the case of the NBD GF,

$$
D_{k}(x)=\frac{x d}{d x}
$$

For this (Qlike) class of equations, we have factorization property

$$
\begin{aligned}
& F=F\left(x_{1}, \ldots, x_{l}\right)=F_{1}\left(x_{1}\right) \ldots F_{l}\left(x_{l}\right), \\
& q^{D_{k}(x)} F_{k}(x)=c_{k} F_{k}(x)^{q}, 1 \leq k \leq l, c_{1} c_{2} \ldots c_{l}=1 .
\end{aligned}
$$

\subsection{NBD motivated equations}

For NBD distribution we have corresponding multiplication(convolution)formulas

$$
\begin{aligned}
& (P \star P)_{n} \equiv \sum_{m=0}^{n} P_{m}(k,<n>) P_{n-m}(k,<n>) \\
& =P_{n}(2 k, 2<n>)=Q_{2} P_{n}(k,<n>), \ldots
\end{aligned}
$$

So, we can say, that star-product on the distributions of NBD corresponds ordinary product for GF.

It will be nice to have similar things for string field theory(SFT) [Kaku, 2000]. 
SFT motion equation is

$$
Q \Phi=\Phi \star \Phi
$$

For stringfield GF F we may have

$$
Q F=F^{2}
$$

By construction we know the solution of the nice equation (10.27) as GF of NBD, F. We obtain corresponding differential equations, if we consider $q=1+\varepsilon$, for small $\varepsilon$,

$$
\begin{aligned}
& \left(D(D-1) \ldots(D-m+1)-(\ln F)^{m}\right) \Psi=0 \\
& \left(\frac{\Gamma(D+1)}{\Gamma(D+1-m)}-(\ln F)^{m}\right) \Psi=0 \\
& \left(D_{m}-\Phi^{m}\right) \Psi=0, m=1,2,3, \ldots \\
& D_{m}=\frac{\Gamma(D+1)}{\Gamma(D+1-m)}, \Phi=\ln F
\end{aligned}
$$

with the solution $\Psi=F=\exp (\Phi)$. In the case of the NBD and p-adic string, we have correspondingly

$$
\begin{aligned}
& D=\frac{x_{1} d}{d x_{1}}+\frac{x_{2} d}{d x_{2}} \\
& D=-\frac{1}{2} \triangle, \triangle=-\partial_{x_{0}}^{2}+\partial_{x_{1}}^{2}+\ldots+\partial_{x_{n-1}}^{2} .
\end{aligned}
$$

These equations have meaning not only for integer $m$.

For high mean multiplicities we have corresponding equations for $\mathrm{KNO}$

$$
\begin{aligned}
& Q_{2} \Psi(z)=\Psi \star \Psi \equiv \int_{0}^{z} \Psi(t) \Psi(z-t) d t \\
& =\left.z \int_{0}^{1} d t t^{\delta_{1}}(1-t)^{\delta_{2}} \Psi\left(z_{1}\right) \Psi\left(z_{2}\right)\right|_{z_{1}=z_{2}=z} \\
& =\left.z \frac{\Gamma\left(\delta_{1}+1\right) \Gamma\left(\delta_{2}+1\right)}{\Gamma\left(\delta_{1}+\delta_{2}+2\right)} \Psi\left(z_{1}\right) \Psi\left(z_{2}\right)\right|_{z_{1}=z_{2}=z}
\end{aligned}
$$

Due to the explicit form of the operator $D$, these equations and corresponding solutions have the symmetry under the change of the variables

$$
k \rightarrow a k,<n>\rightarrow b<n>\text {. }
$$

When

$$
a=\frac{<n>}{k}, b=\frac{k}{<n>}
$$

we obtain the symmetry with respect to the transformations $k \leftrightarrow<n>, x_{1} \leftrightarrow x_{2}$. 


\subsection{The Theory of Invariants with some applications}

The effective action of any field theory in classical, static and homogeneous approximation reduce to the effective potential of the polynomial type, stable critical points of which give us the ground states of corresponding physical system. In the case of one field, for critical points we have usual polynomial equation

$$
P_{n}(x)=a_{n} x^{n}+\ldots+a_{1} x+a_{0}=0
$$

We know that the solutions of this equation is given in the class of algebraic function for $n \leqslant 4$ and can be expressed in terms of the $\theta$-functions for $n \geq 5$, [Mumford, 1983].

In the field of complex numbers, $x \in \mathbf{C}$, we have for $P_{n}(x)=0, n$ solutions, we have $n$ roots $x_{k}, 1 \leq k \leq n$. In the regular case, the roots are different, $x_{n} \neq x_{m}, n \neq m$. In the singular or degenerate case, some of the roots coincides, we have multiple root, we have only $k \leq n$ different roots $x_{m}, 1 \leq$ $m \leq k$, with multiplicity $n_{m}$, of root $x_{m}, n \geq n_{m} \geq 1, n_{1}+n_{2}+\ldots+n_{k}=n$.

Let us start, for warmup from simplest considerations. If we have linear equation,

$$
k x-a=0,
$$

when $k \neq 0$, we have the following unique solution

$$
x=\frac{a}{k} .
$$

If $a \neq 0$ and $k=0$, we have not a solution. And the last case: if $k=a=0$, any $x$ is solution.

Now, we consider the simplest case of deformation, which we will use than for higher order equations too. Let us consider second order equation which will approximate, is deformation, the previous one

$$
\varepsilon x^{2}-k x+a=0, k x-a=\varepsilon x^{2},
$$

This equation will serve us as exactly solvable example on which we will illustrate the perturbation theory method usable for higher dimensional equations. The well known solution is

$$
x=\frac{k \pm \sqrt{k^{2}-4 a \varepsilon}}{2 \varepsilon}=\frac{k\left(1 \pm\left(1-2 a \varepsilon / k^{2}+O\left(\varepsilon^{2}\right)\right)\right.}{2 \varepsilon}
$$

So the minus-sign solution

$$
x_{-}=\frac{a}{k}+O(\varepsilon)
$$

is the deformation of the original solution. The plus-sign solution

$$
x_{+}=\frac{k}{\varepsilon}+O(\varepsilon)
$$

is the extra, pseudo solution, which disappear from any finite region for rather small $\varepsilon$.

Already for second order equation we have both, the regular case, with two different solutions and the singular case, when we have one solution with multiplicity 2 . We will see that by deformation method we can transform the singular case into regular one. Than we answer the question 
how regular case reduce in the limit of zero deformation to the singular case and regular solutions become singular one. The general second order equation is

$$
x^{2}-2 k x+c=0
$$

Its solution is

$$
x=k \pm \sqrt{k^{2}-c}
$$

They are slightly different form of the second order equation with small parameter and its solution considered before as deformation of the first order equation. In regular case, $D=k^{2}-c \neq 0$ and we have two different solutions. In the singular case, $D=0$ and we have one solution with multiplicity 2. Now we make deformation of the singular equation

$$
(x-k)^{2}-\varepsilon x^{3}=0 .
$$

In this case we can also use the known exact solution of the third-order equation, but we prefer the approximate perturbation theoretic method which is applicable also for higher order equations. We try to find a solution which is a small deformation of the singular solution in the following form

$$
x=k+\varepsilon^{a} x_{1}
$$

Put this anzac into deformed equation, than we find

$$
\begin{aligned}
& \varepsilon^{2 a} x_{1}^{2}-\varepsilon k^{3}+O\left(\varepsilon^{1+a}\right)=0 \Rightarrow a=\frac{1}{2}, x_{1}= \pm k^{3 / 2} \\
& x=k \pm \varepsilon^{1 / 2} k^{3 / 2}+O\left(\varepsilon^{3 / 2}\right)
\end{aligned}
$$

So after deformation we obtain instead of one degenerate solution two regular one.

Now let us consider deformation of the regular case,

$$
\begin{aligned}
& x^{2}-2 k x+c-\varepsilon x^{3}=0, D=k^{2}-c \neq 0, \\
& \left(x-x_{0+}\right)\left(x-x_{0-}\right)-\varepsilon x^{3}=0 \\
& x_{0 \pm}=k \pm \sqrt{k^{2}-c} \\
& x_{ \pm}=x_{0 \pm}+\varepsilon x_{1 \pm} \\
& x_{1+}\left(x_{0+}-x_{0-}\right)-x_{0+}^{3}=0, \\
& x_{1+}=\frac{x_{0+}^{3}}{x_{0+}-x_{0-}}=\frac{x_{0+}^{3}}{2 \sqrt{k^{2}-c}}, x_{1-}=-\frac{x_{0-}^{3}}{x_{0+}-x_{0-}}
\end{aligned}
$$

Regular case corresponds to the deformation of the singular one, when

$$
x_{1+}=\frac{k^{3}}{\sqrt{k^{3} \varepsilon}}, k^{2}-c=k^{3} \varepsilon / 4
$$

Next case is third-order equation,

$$
P_{3}(x, a)=x^{3}+a_{2} x^{2}+a_{1} x+a_{0}=0,
$$

With forth-order deformation,

$$
P_{3}-\varepsilon x^{4}=0
$$


In the most singular case we have one root with multiplicity 3 , which transforms into three regular roots after deformation:

$$
\begin{aligned}
& \left(x-x_{0}\right)^{3}-\varepsilon x^{4}=0, \\
& x_{k}=x_{0}+\varepsilon^{1 / 3} x_{0}^{4 / 3} \exp \left(\frac{2 \pi}{3}(k-1)\right), k=1,2,3
\end{aligned}
$$

Now it is obvious the deformation picture in the case of general polynomial equation with one root of multiplicity $n$ :

$$
\begin{aligned}
& \left(x-x_{0}\right)^{n}-\varepsilon x^{n+1}=0, \\
& x_{k}=x_{0}+\varepsilon^{1 / n} x_{0}^{(n+1) / n} \exp \left(\frac{2 \pi}{n}(k-1)\right), k=1,2, \ldots, n-1 .
\end{aligned}
$$

Now consider semi-singular (or semi-regular, as you like to name this case) case, when one have singular and regular roots. The simplest example, when we have one regular and one singular roots, is

$$
\begin{aligned}
& (x-a)^{2}(x-b)-\varepsilon x^{4}=0, \\
& x_{ \pm}=a \pm \varepsilon^{1 / 2} \frac{a^{2}}{\sqrt{a-b}} \\
& x_{3}=b+\varepsilon \frac{b^{4}}{(a-b)^{2}}
\end{aligned}
$$

we see that the $\varepsilon$-dependence of the deformed values of the roots are similar with previous examples.

For the deformation of the corresponding regular equation we have

$$
\begin{aligned}
& \left(x-e_{1}\right)\left(x-e_{2}\right)\left(x-e_{3}\right)-\varepsilon x^{4}=0, \\
& x_{1}=e_{1}+\varepsilon \frac{e_{1}^{4}}{\left(e_{1}-e_{2}\right)\left(e_{1}-e_{3}\right)}
\end{aligned}
$$

The singular case corresponds to $e_{i}-e_{j} \sim \varepsilon^{1 / 3} x_{0}^{4 / 3}$

\subsection{Numerical methods of finding roots}

Finding of the real numerical values of the simple roots is not a problem with computer. Near a simple root $x_{k}$ a polynomial $P_{n}(x)$ has form

$$
P_{n}(x)=\left(x-x_{k}\right) P_{n-1}\left(x_{k}\right)+O\left(\left(x-x_{k}\right)^{2}\right),
$$

so it changes sign in a small interval containing $x_{k}$ and dividing the interval in two, usually by middle point, parts, we find in which of them is the root, repetition of this procedure gives exponentially quickly convergent approximations to the root.

In the case of the multiple zeros,

$$
P_{n}(x)=\left(x-x_{k}\right)^{m} P_{n-m}\left(x_{k}\right)+O\left(\left(x-x_{k}\right)^{m+1}\right), m \geq 2,
$$


it is not easy to find multiplicity of the root when it is odd integer and it is difficult to find the root and its multiplicity when multiplicity is even integer number. In the way of developing effective numerical algorithms and computer programs, we note that, when we have good procedure of finding logarithmic derivative of the polynomial,

$$
\frac{P_{n}(x)^{\prime}}{P_{n}(x)}=\left(\ln P_{n}(x)\right)^{\prime}=\sum_{k} \frac{m_{k}}{x-x_{k}}, \sum_{k} m_{k}=n,
$$

we can find roots as resonances in particle physics spectrum of crosssections.

\subsection{Quantum groups with some applications}

Deformation or an intelligent introduction of the new parameters is the useful concept in theoretical physics and mathematics. Quantum mechanics may be considered as a deformation (the deformation parameter being $\hbar$ ) of classical mechanics and relativistic mechanics is, to a certain extent, another deformation (with $1 / \mathrm{c}$ as deformation parameter) of classical mechanics. In a sense a three dimensional space $(\hbar, 1 / c, G)$, where $G$ is Newton's gravitational constant defines a theories space. In this space the points: $(0,0, G),(0,1 / c, 0),(\hbar, 0,0),(\hbar, 1 / c, 0),(\hbar, 0, G),(\hbar, 1 / c, G)$ denote correspondingly: classical gravitation, special theory of relativity, quantum mechanics, quantum field theories, non-relativistic quantum gravity and M-theory. It is natural to travel in the theories space by renormdynamics with running parameters.

The concept of a quantum algebra (or quantum group) was introduced, under different names, by Kulish, Reshetikhin,Sklyanin,(from the Faddeev school), Drinfeld and Jimbo in terms of a quantized universal enveloping algebra or an Hopf bi-algebra and, independently, by Woronowicz in terms of a compact matrix pseudo-group [Kulish et al, 1981-87].

Among the various motivations that led to the concept of a quantum group, we have to mention the quantum inverse scattering technique, the solution of the quantum Yang-Baxter equation and, more generally, the study of exactly solvable models in statistical mechanics; conformal field theories in two dimensions ; (quantum) dynamical systems ; quantum optics ; molecular, atomic and nuclear spectroscopies ; condensed matter physics ; knot theory, theory of link invariants (Jones polynomials) and braid groups ; and so on. In addition, the concept of a quantum group constitutes a basic tool in non-commutative geometry. Thus, quantum groups are of paramount importance not only in physics and quantum chemistry but in pure mathematics equally well.

\subsection{1 pq-oscillators}

We define the state vectors $\mid n>$ from (one-particle) Fock space $F=\{\mid n>, n=0,1,2, \ldots\}$ as eigenstates for an ordinary harmonic oscillator in one dimension; the linear operators $a, a^{+}$and $N$ on the vector space $F$ by the relations

$$
a^{+}|n>=\sqrt{[n+1]}| n+1>, a|n>=\sqrt{[n]}| n-1>, a|0>=0, N| n>=n \mid n>
$$

where we use the notation

$$
[c]=\frac{p^{c}-q^{c}}{p-q}, c \in \mathbb{C},
$$

the two parameters $p$ and $q$ being fixed parameters taken in the field of complex numbers $\mathbb{C}$. 
In the limiting case $p=q^{-1} \rightarrow 1$, we have simply $[c]=c$ so that $a^{+}, a$ and $N$ are (respectively) in this case the ordinary creation, annihilation and number operators. Observe that the operator $N$ is a non-deformed operator that coincides with the usual number operator. Two particular situations are of special interest, viz., $p=q^{-1}$, which is mainly encountered in the physical literature and $p=1$, which comes from the mathematical literature. A simple iteration of equation (25.774) yields

$$
\left|n>=\frac{\left(a^{+}\right)^{n}}{\sqrt{[n] !}}\right| 0>,[n] !=[n][n-1] \ldots[1], n \in \mathbb{N},[0] !=1
$$

We have

$$
(a)^{+}=a^{+},(N)^{+}=N,\left[N, a^{+}\right]=a^{+},\left[N, a^{-}\right]=-a^{-}
$$

where $(X)^{+}$denotes the adjoint of the operator $X$ and $[A, B]=[A, B]_{-}=A B-B A$.

Indeed, e.g.

$$
\left[N, a^{+}\right]|n>=\sqrt{[n+1]}(n+1)| n+1>-n \sqrt{[n+1]}\left|n+1>=a^{+}\right| n>
$$

Equation $(a)^{+}=a^{+}$is valid under the condition that the pq-deformed numbers $[n], n \in \mathbb{N}$, are real ; this is certainly the case if $p$ and $q \in \mathbb{R}$ or $p=q^{-1} \in S^{1}{ }^{2}$

We have the following basic property

$$
a a^{+}=[N+1], a^{+} a=[N]
$$

Indeed,

$$
a a^{+}|n>=a \sqrt{[n+1]}| n+1>=[n+1]|n>=[N+1]| n>, \forall n
$$

As a corollary of the basic property we have the following expression for the $c$-mutator

$$
\left[a, a^{+}\right]_{c}=a a^{+}-c a^{+} a=\frac{p^{N}(p-c)-(q-c) q^{N}}{p-q}, c \in \mathbb{C}
$$

For $p=1$, we have

$$
\left[a, a^{+}\right]_{q}=1,\left[a, a^{+}\right]_{1}=q^{N}
$$

that correspond to the deformed bosons encountered mainly in the mathematical literature. Note that the relation $\left[a, a^{+}\right]_{q}=1$, which interpolates between fermions and bosons for $-1 \leq q \leq 1$, may be of interest in anyonic statistics and construction of the models with small deviation from Pauli (exclusion) principle.

\subsection{Coherent states description (CSD)}

CSD at a point $x$ we define as

$$
\hat{a}|x>=x| x>, \hat{a}=\frac{i}{\sqrt{2}}(\hat{x}-i \hat{p})=\frac{1}{\sqrt{2}}(\hat{p}+i \hat{x}), \hat{a}^{+}=\frac{1}{\sqrt{2}}(\hat{p}-i \hat{x}),\left[\hat{a}, \hat{a}^{+}\right]=1,
$$

\footnotetext{
${ }^{2}$ For $p=q^{-1}=e^{i \varphi} \in S^{1}, \varphi \in \mathbb{R},[n+1]=\frac{\sin (n \varphi+\varphi)}{\sin \varphi}=U_{n}(\cos \varphi)$-the Chebyshev polynomial of the second kind.
} 


\subsection{Pauli exclusion principle and its small violation}

The Pauli exclusion principle is the quantum mechanical principle that no two identical fermions (particles with half-integer spin) may occupy the same quantum state simultaneously. A more rigorous statement is that the total wave function for two identical fermions is anti-symmetric with respect to exchange of the particles. The principle was formulated by Austrian physicist Wolfgang Pauli in 1925.

Integer spin particles, bosons, are not subject to the Pauli exclusion principle: any number of identical bosons can occupy the same quantum state, as with, for instance, photons produced by a laser and BoseÜEinstein condensate.

According to the spin-statistics theorem, particles with integer spin occupy symmetric quantum states, and particles with half-integer spin occupy antisymmetric states; furthermore, only integer or half-integer values of spin are allowed by the principles of quantum mechanics. In relativistic quantum field theory, the Pauli principle follows from applying a rotation operator in imaginary time to particles of half-integer spin. Since, nonrelativistically, particles can have any statistics and any spin, there is no way to prove a spin-statistics theorem in nonrelativistic quantum mechanics.

In one dimension, bosons, as well as fermions, can obey the exclusion principle. A onedimensional Bose gas with delta function repulsive interactions of infinite strength is equivalent to a gas of free fermions. The reason for this is that, in one dimension, exchange of particles requires that they pass through each other; for infinitely strong repulsion this cannot happen. This model is described by a quantum nonlinear Schrodinger equation. In momentum space the exclusion principle is valid also for finite repulsion in a Bose gas with delta function interactions [Zzergin, Korepin, 1982], as well as for interacting spins and Hubbard model in one dimension, and for other models solvable by Bethe ansatz. The ground state in models solvable by Bethe ansatz is a Fermi sphere.

The Pauli Exclusion Principle (PEP) uncovered by Wolfgang Pauli in 1925 [Pauli, 1925] is one of the corner stones of quantum physics and thus it is at the basis of the foundation of modern physics. It is connected with spin statistics dividing the world in fermions and bosons. Therefore, PEP is one of the most important rules in physics. Based on it is our understanding of nature and the consequences for the world of elementary particles up to compact objects (e.g. neutron stars) in the universe - but it is lacking a simple explanation as already stated by Pauli himself.

Wolfgang Pauli showed later in 1940 the interconnection of the principle with the spin-statistics [Pauli, 1940]. We know that the Pauli Principle is extremely well fulfilled but the the limit of validity - if any - is still an open question. Even a tiny violation of PEP would point to new physics which could show up at very high energies (at the Planck scale).

\subsection{Fermion vacuum condensate and Ultracold neutrons}

It was Enrico Fermi who realized first that the coherent scattering of slow neutrons would result in an effective interaction potential for neutrons traveling through matter, which would be positive for most materials [Fermi, 1936]. The storage of neutrons with very low kinetic energies was predicted by Yakov Borisovich Zel'dovich [Zeldovich, 1959] and experimentally realized simultaneously by groups at Dubna [Lushikov, 1969] and Munich [Steyerl, 1969]. 
Ultracold neutrons (UCN) are free neutrons which can be stored in traps made from certain materials. The storage is based on the reflection of UCN by such materials under any angle of incidence. The reflection is caused by the coherent strong interaction of the neutron with atomic nuclei. It can be quantum-mechanically described by an effective potential which is commonly referred to as the Fermi pseudo potential or the neutron optical potential. The corresponding velocity is called the critical velocity of a material. Neutrons are reflected from a surface if the velocity component normal to the reflecting surface is less or equal the critical velocity. As the neutron optical potential of most materials is below $300 \mathrm{neV}$, the kinetic energy of incident neutrons must not be higher than this value to be reflected under any angle of incidence, especially for normal incidence. The kinetic energy of $300 \mathrm{neV}$ corresponds to a maximum velocity of $7.6 \mathrm{~m} / \mathrm{s}$ or a minimum wavelength of 52 $\mathrm{nm}$. As their density is usually very small, UCN can also be described as a very thin ideal gas with a temperature of $3.5 \mathrm{mK}$. Due to the small kinetic energy of an UCN, the influence of gravitation is significant. Thus, the trajectories are parabolic. Kinetic energy of an UCN is transformed into potential (height) energy with $\sim 102 \mathrm{neV} / \mathrm{m}$. The magnetic moment of the neutron, produced by its spin, interacts with magnetic fields. The total energy changes with $\sim 60 \mathrm{neV} / \mathrm{T}$.

Any material with a positive neutron optical potential can reflect UCN. The height of the neutron optical potential is isotope-specific. The highest known value of $V_{F}$ is measured for ${ }^{58} \mathrm{Ni}$ : $335 n e V\left(v_{C}=8.14 m / s\right)$. It defines the upper limit of the kinetic energy range of UCN. The most widely used materials for UCN wall coatings are Beryllium, Beryllium oxide, Nickel and more recently also diamond-like carbon (DLC), $V_{F}=304 \mathrm{neV}, v_{C}=7.65 \mathrm{~m} / \mathrm{s}$. Non-magnetic materials such as DLC are usually preferred for the use with polarized neutrons. Magnetic centers in e.g. Ni can lead to depolarization of such neutrons upon reflection. If a material is magnetized, the neutron optical potential is different for the two polarizations, caused by

$$
V_{F}=V_{0} \pm \mu B
$$

where $\mu$ is the magnetic moment of the neutron and $B=\mu_{0} M$ the magnetic field created on the surface by the magnetization.

Each material has a specific loss probability per reflection,

$$
\eta(E, \theta)=\eta \sqrt{\frac{E \cos ^{2} \theta}{V_{F}-E \cos ^{2} \theta}}
$$

which depends on the kinetic energy of the incident UCN (E) and the angle of incidence $(\theta)$. It is caused by absorption and thermal upscattering. The loss coefficient $\eta$ is energy-independent and typically of the order of $10^{-4}$ to $10^{-3}$. The production, transportation and storage of UCN is currently motivated by their usefulness as a tool to determine properties of the neutron and to study fundamental physical interactions. Storage experiments have improved the accuracy or the upper limit of some neutron related physical values.

The neutron electric dipole moment (nEDM) is a measure for the distribution of positive and negative charge inside the neutron. No nEDM has been found until now. Today's lowest value for the upper limit of the nEDM was measured with stored UCN. 


\subsection{Quantum vortex trapping of UCN}

In physics, a quantum vortex is a topological defect exhibited in superfluids and superconductors. The existence of these quantum vortices was predicted by Lars Onsager in 1947 in connection with superfluid Helium. Onsager also pointed out that quantum vortices describe circulation of superfluid and conjectured that their excitations are responsible for superfluid phase transition. These ideas of Onsager were further developed by Richard Feynman in 1955 [Feynman, 1955] and in 1957 were applied to describe magnectic phase diagram of type-II superconductors by Alexei Alexeyevich Abrikosov [Abrikosov, 1957]. Quantum vortices are observed experimentally in Type-II superconductors, liquid helium, and atomic gases (in Bose-Einstein condensate). In a superfluid, a quantum vortex "carries" quantized angular momentum, thus allowing the superfluid to rotate; in a superconductor, the vortex carries quantized magnetic flux.

In a superfluid, a quantum vortex is a hole with the superfluid circulating around the vortex; the inside of the vortex may contain excited particles, air, vacuum,..., UCNs, etc. The thickness of the vortex depends on a variety of factors; in liquid helium, the thickness is on the order of a few Angstroms. A superfluid has the special property of having phase, given by the wavefunction, and the velocity of the superfluid is proportional to the gradient of the phase. The circulation around any closed loop in the superfluid is zero, if the region enclosed is simply connected. The superfluid is deemed irrotational. However, if the enclosed region actually contains a smaller region that is an absence of superfluid, for example a rod through the superfluid or a vortex, then the circulation is,

$$
\oint v \cdot d l=\frac{1}{m} \oint p \cdot d l=\frac{\hbar}{m} \oint \nabla \phi \cdot d l=\frac{\hbar}{m} \triangle \phi
$$

where $m$ is the mass of the superfluid particle, and $\phi$ is the phase difference around the vortex. Because the wavefunction must return to its same value after an integral number of turns around the vortex (similar to what is described in the Bohr model), then $\triangle \phi=2 \pi n$, where $\mathrm{n}$ is an integer. Thus, we find that the circulation is quantized:

$$
\oint v \cdot d l=\frac{\hbar}{m} 2 \pi n=\frac{h}{m} n
$$

where $h$ is Planck's constant.

\subsection{Vortex in a superconductor}

A principal property of superconductors is that they expel magnetic fields; this is called the Meissner effect. If the magnetic field becomes sufficiently strong, one scenario is for the superconductive state to be "quenched". However, in some cases, it may be energetically favorable for the superconductor to form a lattice of quantum vortices, which carry quantized magnetic flux through the superconductor. A superconductor that is capable of having vortex lattices is called a type-II superconductor. Over some enclosed area $\mathrm{S}$, the magnetic flux is

$$
\Phi=\oint_{\partial S} A \cdot d l=\oint_{S} B \cdot n d^{2} x
$$

Substituting a result of London's equation:

$$
j_{s}=-n_{s} \frac{e_{s}^{2}}{m} A-n_{s} \frac{e_{s} \hbar}{m} \nabla \phi,
$$


we find

$$
\Phi=-\frac{m}{n_{S} e_{S}^{2}} \oint_{\partial S} j_{S} \cdot d l-\frac{\hbar}{e_{S}} \oint_{\partial S} \nabla \phi \cdot d l
$$

where $n_{s}, m$, and $e_{s}$ are the number density, mass and charge of the Cooper pairs. If the region $S$, is large enough so that $j_{s}=0$ along $\partial S$, then

$$
\Phi=-\frac{\hbar}{e_{s}} \oint_{\partial S} \nabla \phi \cdot d l=-\frac{\hbar}{e_{s}} \oint_{S} \triangle \phi=\frac{h}{e_{s}} n
$$

The flow of current can cause vortices in a superconductor to move, it causes the electric field due to the phenomenon of electromagnetic induction. This leads to energy dissipation and causes the material to display a small amount of electrical resistance while in the superconducting state.

\subsection{Quantum levels for UCN in gravitational field}

Physicists have observed quantized states of matter under the influence of gravity for the first time. Valery Nesvizhevsky of the Institute Laue-Langevin and colleagues found that cold neutrons moving in a gravitational field do not move smoothly but jump from one height to another, as predicted by quantum theory. The finding could be used to probe fundamental physics such as the equivalence principle, which states that different masses accelerate at the same rate in a gravitational field (V Nesvizhevsky et al. 2001 Nature 415 297).

\subsection{Quanputing and non-locality in quantum mechanics}

Well-known examples of non-locality in quantum mechanics are EPR pairs [Einstein, Podolsky, Rosen, 1935] and the GHZ state [Greenberger, Horne, Zeilinger, 1989]. States of quantum codes contradict local realism, too [DiVincenzo, Peres, 1997]. One common feature of these states is that the non-local properties do not change under local transformations, i. e., unitary operations acting independently on each of the subsystems. Thus, any function invariant under local unitary transformations (LUT) can be used to describe these non-local properties [Schlienz, Mahler, 1995, Schlienz, Mahler, 1996].

\subsection{Entanglement entropy (EE)}

Entanglement is a fundamental phenomena in a wide variety of areas ranging from quantum information and condensed matter physics, [Entang]1] to quantum gravity and string theory, [Entangl2]

\subsection{AdS/CFT correspondence and Holographic Rényi entropy}

The tree level contribution and 1-loop correction of Rény holographic entanglement entropy (RHE) exactly match the direct CFT computation in the large central charge limit. This allows the Rény entanglement entropy to be a new window to study the AdS/CFT correspondence. 


\subsection{Constituent Nature of Particles and Coupling Constant Numbers}

In one copling constant-one dimensional renormdynamics, dimensional quantities, like hadron masses in QCD, have the following dependence on the coupling constant

$$
m=\Lambda \exp (-a / \alpha(\mu))
$$

Now, if the inverse fine structure constant $\alpha^{-1}=N=n_{1} n_{2}=\alpha_{1}^{-1} \alpha_{2}^{-1}$ is integer but not prime number than we have the following relations

$$
\begin{aligned}
& m=\Lambda \exp \left(-a_{1} / \alpha_{1}\left(\mu_{1}\right)\right)=\Lambda \exp \left(-a_{2} / \alpha_{2}\left(\mu_{2}\right)\right), \\
& a_{1}=n_{2} a=2 \pi / b_{1}, a_{2}=n_{1} a=2 \pi / b_{2}, \mu>\mu_{1}>\mu_{2}, n_{1}>n_{2}, \\
& a=2 \pi / b, b=\frac{11}{3} n_{c}-\frac{2}{3} n_{f}, b_{1}=\frac{11}{3} \frac{n_{c}}{n_{2}}-\frac{2}{3} \frac{n_{f}}{n_{2}}, b_{2}=\frac{11}{3} \frac{n_{c}}{n_{1}}-\frac{2}{3} \frac{n_{f}}{n_{1}} \\
& \alpha=\frac{g^{2}}{4 \pi}=1 / N, \alpha<\alpha_{1}<\alpha_{2}, \alpha_{1}=1 / n_{1}, \alpha_{2}=1 / n_{2}, N=n_{1} n_{2}
\end{aligned}
$$

In 123-SM, we have three coupling constants, $\alpha_{1}, \alpha_{2}, \alpha_{3}$. If at a scale $m$, they have inverse integer values with common factor: $\alpha_{k}^{-1}=n n_{k}$, than

$$
\exp \left(-a_{k} / \alpha_{k}(m)\right)=\exp \left(-A_{k} / \alpha(M)\right), A_{k}=n_{k} a_{k}, \alpha(M)^{-1}=n
$$

where at the scale $M$ we have unified theory with fine structure coupling constant $\alpha(M)$. In MSSM $\alpha(M)^{-1}=25, M=10^{16} \mathrm{GeV}$, [Weinberg, 2000], [Kazakov, 2004].

We can extract also from some $b_{k}$ an integer factor and include it into $\alpha_{k}$, hence decrease their values.

\subsection{String-Field Duality}

In the relativistic string-gauge field duality [Maldacena, 1999] (see review [Aharony et al, 2000]), the string coupling constant $g_{s}$ and the gauge field fine structure constant $\alpha_{s}$ are related: $g_{s}=\alpha_{s}$. The statement that the later is (prime) integer means (prime) integer quantization of the string coupling constant.

\subsection{Instantons}

Instanton configuration has the following value of classical action

$$
S[A]=\int d^{4} x \frac{1}{4}\left(G_{\mu \nu}^{a}\right)=\frac{8 \pi^{2}}{g^{2}}=\frac{2 \pi}{\alpha}=2 \pi p, \alpha=p^{-1}
$$

So, in Minkowski space we have not destructive interference between instanton contributions

$$
e^{i S}=1
$$

when $\alpha=p^{-1}$. When $\alpha=p$, we need at least $p$ instantons in a cluster-molecule, we have cumulative action of $p$ instantons. So, on the valence quark scale, gluon fields are presented implicitly as instanton clusters. When $p=2$, we have instanton dipoles. 


\subsection{Instantons of $G_{2}$ gauge theory}

Yang-Mills theory and QCD are well-defined theories for an arbitrary (semi-)simple Lie group as gauge group. One remarkable choice for the group is the exceptional Lie group $G_{2}$ instead of the physical group $S U$ (3). Since its center is trivial the Wilson confinement criterion is not fulfilled, even in the pure Yang-Mills case. The reason is that any static fundamental charge can be screened by three adjoint charges, i. e. gluons. The most remarkable difference compared to the $S U(3)$ or $S U(2)$ case is the topological charge of the $G_{2}$ instanton [Ernst-Michael Ilgenfritz, Axel Maas, 2012]

$$
Q=\frac{1}{64 \pi^{2}} \int d^{4} x F_{\mu \nu}^{a} \varepsilon_{\mu v \rho \sigma} F_{\rho \sigma}^{a}=2
$$

which is twice as large as the one of the (embedded) $S U(2)$ instanton.

\subsection{Topological Field Theories}

In the case of the $N=4$ super Yang-Mills the moduli space is the upper half plane parametrized by

$$
\tau=\frac{\theta}{2 \pi}+i \frac{4 \pi}{g^{2}}=\frac{\theta}{2 \pi}+\frac{i}{\alpha}=\frac{1}{2 \pi}\left(\theta+i \frac{2 \pi}{\alpha}\right)
$$

\subsection{Nekrasov Instanton Partition Function}

The instanton part of the partition function is given by

$$
Z=\sum_{k \geq 0} z_{k} q^{k}
$$

where instanton parameter $q$ is given by

$$
q=e^{2 \pi \tau i}=e^{-\frac{8 \pi^{2}}{g^{2}}+i \theta}, \tau=\frac{\theta}{2 \pi}+i \frac{4 \pi}{g^{2}}
$$

\subsection{Gluodynamics with the $\theta$-term}

The Lagrangian is

$$
\begin{aligned}
& L=-\frac{1}{4} F_{\mu \nu}^{a} F_{\mu \nu}^{a}+\theta \frac{g^{2}}{8 \pi^{2}} F_{\mu \nu}^{a} \tilde{F}_{\mu \nu}^{a}=\frac{1}{2}\left(\mathbf{E}^{2}-\mathbf{B}^{2}\right)+\frac{\theta \alpha}{2 \pi} \mathbf{E} \cdot \mathbf{B}, \\
& \tilde{F}_{\mu \nu}^{a}=\frac{1}{2} \varepsilon_{\mu \nu \rho \sigma} F_{\rho \sigma}^{a}
\end{aligned}
$$

The $\theta$-term is a total derivative and does not contribute to the classical equation of motion. It does, however, change the canonical momentum from $P_{n}^{a}=F_{0 n}^{a}$ to

$$
P_{n}^{a}=F_{0 n}^{a}+\theta \frac{g^{2}}{8 \pi^{2}} B_{n}^{a}, n=1,2,3
$$

and after canonical quantization in Weyl gauge one finds the Hamiltonian $H_{\theta}$

$$
H_{\theta}=\int d^{3} x\left(\frac{1}{2}\left(P_{n}^{a}-\theta \frac{g^{2}}{8 \pi^{2}} B_{n}^{a}\right)^{2}+\frac{1}{2}\left(B_{n}^{a}\right)^{2}\right)
$$




\subsection{The $U(1)_{A}$ Anomaly and QCD Phenomenology}

An important historical role in establishing QCD as the theory of the strong interactions was played by $U(1)_{A}$ anomaly.The description of radiative decays of the pseudoscalar mesons in the framework of a gauge theory requires the existence of the electromagnetic axial anomaly and determines the number of colours to be $N_{c}=3$. The compatibility of the symmetries of QCD with the absence of a ninth light pseudoscalar meson, the so called $U(1)_{A}$ problem, in turn depends on the contribution of the colour gauge fields to the anomaly. The anomaly-mediated link between quark dynamics and gluon topology (the non-perturbative dynamics of topologically nontrivial gluon configurations) is the key to understanding a range of phenomena in polarised QCD phenomenology, most notably the 'proton spin' sum rule for the first moment of the structure function $g_{1 p}$. A wide variety of phenomena in QCD, ranging from the low-energy dynamics of the pseudoscalar mesons to sum rules in polarised deep-inelastic scattering reveal subtle aspects of quantum field theory, in particular topological gluon dynamics, which go beyond simple current algebra or parton model interpretations.

\subsection{Low Energy Supersymmetry of Nucleons in the Pion Condensate Medium}

The pseudoscalar pion-nucleon interaction model at low energy and external pion condensate field reduce to the model given by Hamiltonian

$$
H=\frac{p^{2}}{2 M}+\frac{g^{2} \pi^{2}}{2 M}+\frac{g}{2 M}(\sigma \nabla)(\tau \pi),
$$

If condensate is electro-neutral, $\pi_{a}=\delta_{a 3} \pi$ then the Hamiltonian have the following supersymmetric form

$$
H=\frac{1}{2 M}\left\{Q^{+}, Q^{-}\right\}, Q^{ \pm}=(\sigma p \pm i g \pi(x)) \tau^{ \pm}
$$

\subsection{Quarkonia, potential and space dimension renormdynamics}

Phenomenological approach to the nonrelativistic potential-model study of $\Upsilon$ and $\psi$ spectra leads to a static Coulombic Power-law potential of the form

$$
\begin{aligned}
V(r)=a(r) r^{2-d(r)} \sim 1 / r, r & \sim 0.1 \mathrm{fm} \\
r, r & \sim 1 . f m
\end{aligned}
$$

E.g. in the case of the $\Upsilon$ and small $r$

$$
V(r)=\frac{4}{3} \frac{\alpha_{s}}{r}, \alpha_{s}=\frac{2 \pi}{b \ln r \Lambda}, b=9 .
$$

This behavior corresponds not only to the running fine structure constant but also to the running space dimension. Confinement-the point-like hadrons on the scales higher than hadronic, corresponds to the zero dimensional space for hadron constituents.

RD equations of QCD beyond the critical dimention has explicit dependence on the space dimension. When the dimension becomes running we should consider two dimensional renormdinamics

$$
a_{1}=\beta_{1}\left(a_{1}, a_{2}\right), a_{1}=a,
$$




$$
\begin{aligned}
& \dot{a}_{2}=\beta_{2}\left(a_{1}, a_{2}\right), a_{2}=d, \\
& \frac{d a}{d d}=\beta(a, d)=\frac{\beta_{1}(a, d)}{\beta_{2}(a, d)}
\end{aligned}
$$

\subsection{Physical models connected to the zeta function}

Consider a gas of massless, relativistic bosons with single-particle energy $E_{k}=|\mathbf{k}|$ in $d$ spatial dimensions, where $k$ is the momentum vector, at a temperature $T=1 / \beta$ and zero chemical potential. The free energy density, which is minus the pressure, is given by the well-known formula:

$$
F=\beta^{-1} \int \frac{d^{D} \mathbf{k}}{(2 \pi)^{D}} \ln \left(1-e^{-\beta|\mathbf{k}|}\right)=-\frac{\Gamma(D)}{2^{D-1} \pi^{\frac{D}{2}} \Gamma\left(\frac{D}{2}\right) \beta^{D+1}} \zeta(D+1), \Re(D)>0
$$

When the boson in question is one polarization of a physical photon, then the above formula leads to Planck's black body spectrum, and this discovery in fact marked the birth of Quantum Mechanics; Planck's constant $\hbar$ was first determined in this way. In performing the above integral we use

$$
\begin{aligned}
& \int d^{D} \mathbf{k}=\Omega_{D} \int_{0}^{\infty} d k k^{D-1}, k=|\mathbf{k}|, \Omega_{D}=\frac{2 \pi^{\frac{D}{2}}}{\Gamma\left(\frac{D}{2}\right)} \\
& \int_{0}^{\infty} d k k^{D-1} \ln \left(1-e^{-k}\right)=-\frac{1}{D} \int_{0}^{\infty} \frac{d k k^{D}}{e^{k}-1}=-\frac{1}{D} \Gamma(D+1) \zeta(D+1), \Re(D)>0
\end{aligned}
$$

In many other physical situations, in order to regularize divergent integrals, one analytically continues certain functions of the dimension $d$ into the complex plane in a procedure referred to as "dimensional regularization". To the zeros of zeta corresponds zeros of free energy. Zeros on critical line corresponds $\Re(D)=-\frac{1}{2}$

\subsection{Solvable model of Renormdynamics}

As a solvable model which will illustrates of the renormdynamics let us consider the following equation

$$
\dot{x}=\beta(x), \beta(x)=\frac{\varepsilon x}{1-\varepsilon x}=\sum_{n \geq 1} \beta_{n} x^{n}, \geq 0, \beta_{n}=\varepsilon^{n}, x \geq 0 .
$$

Here $\beta(x) \leq 0$, for $\varepsilon<0$, and $x$ decries with time monotonically. For $\varepsilon>0$, and $x<1 / \varepsilon(x>1 / \varepsilon)$, $\beta(x) \geq 0(\beta(x) \leq 0)$, and $x$ increase (decries) with time. From the equation we find

$$
\frac{1-\varepsilon x}{\varepsilon x} d x=d t \Rightarrow x^{1 / \varepsilon} e^{-x}=c e^{t} \Rightarrow x e^{-\varepsilon x}=c^{\varepsilon} e^{\varepsilon t}, t=\frac{1}{\varepsilon} \ln x-x-\ln c
$$

So, we have an implicit form of the solution, $t=f(x), x=f^{-1}(t)$. More realistic example is

$$
\beta_{n}=(-1)^{n} n !, \beta(x)=\Gamma\left(\delta_{x}+1\right) \sum_{n \geq 1}(-x)^{n}=\int_{0}^{\infty} d t e^{-t} \frac{-t x}{1+t x}=\int_{0}^{\infty} d t \frac{e^{-t}}{1+t x}-1
$$




\subsection{Solvable Models of QCD Renormdynamics}

The renormdynamic properties of Quantum Chromodynamics were the reason of acceptance of this theory as the theory of strong interactions. The central role played by the QCD $\beta$-function, calculated at the one-[it Hooft, 1972], [Gross, Wilczek, 1973], [Politzer, 1973], two-[Caswell, 1974], [Dones, 1974], [Egorian,Tarasov, 1979], three-[Tarasov, Vladimirov,Zharkov, 1980], [Larin, Vermaseren, 1993] and finally at the four-loop [van Ritbergen,Vermaseren,Larin,1997] level, cannot be overestimated in this respect.

The minimal form of the QCD renormdynamics $(\mathrm{RD})$ is

$$
\begin{aligned}
& \dot{x}=-b_{2} x^{2}-b_{3} x^{3}, \\
& b_{2}=11-\frac{2}{3} n, b_{3}=2\left(51-\frac{19}{3} n\right), x=\frac{\alpha_{s}}{4 \pi}=\left(\frac{g}{4 \pi}\right)^{2},
\end{aligned}
$$

where $n$ is the number of the light quarks,e.g. $n=3$ for energy scales less then the mass of the $c$-quark, $m_{c} \simeq 1 \mathrm{GeV}$ but higher than the mass of $s-$ quark, $m_{s} \simeq 100 \mathrm{MeV}$.

Let us consider the following model of QCD RD

$$
\begin{aligned}
& \dot{x}=-(c \sin (d x))^{2}=-b_{2} x^{2}+\ldots, b_{2}=(c d)^{2}, d x_{0}=d \frac{2}{4 \pi}=\pi \Rightarrow d=2 \pi^{2}, c=\frac{\sqrt{b_{2}}}{d}, \\
& \cot (d x)=c^{2} d t+\text { const } \Rightarrow x(t)=\frac{1}{d}\left(\frac{\pi}{2}-\arctan \left(c^{2} d t\right)\right), \\
& x(-\infty)=\frac{\pi}{d}, \alpha_{s}(-\infty)=\frac{4 \pi^{2}}{d}=2, \alpha_{s}(\infty)=0 .
\end{aligned}
$$

So, this model describes ultraviolet and infrared asymptotics of QCD, but it has not the nontrivial (and invariant) second term of the perturbative RD. Note that, for non-integer (effective) value $n$

$$
n=n^{*}=\frac{3 \cdot 51}{19}=8+\varepsilon, \varepsilon=1 / 19, b_{3}=0,
$$

and we have complete agreement with the minimal model of QCD RD, because there is a parametrization were the solvable model is exact one. For a general compact simple Lie group,

$$
\begin{aligned}
& b_{2}=\frac{11}{3} C_{A}-\frac{4}{3} T_{F} n \\
& b_{3}=\frac{34}{3} C_{A}^{2}-\left(\frac{20}{3} C_{A}+4 C_{F}\right) T_{F} n,
\end{aligned}
$$

where, $C_{A}$ and $C_{F}$ are quadratic Casimir operators of the adjoint and fundamental representations; $T_{F}$ is the normalization of the fundamental representation generators $T^{a}$,

$$
\operatorname{tr}\left(T^{a} T^{b}\right)=T_{F} \delta^{a b}
$$

$n$ is the number of fermion families. In the case of the $S U(N)$ gauge group,

$$
C_{A}=N, C_{F}=\frac{N^{2}-1}{2 N}, T_{F}=\frac{1}{2}
$$

The two loop contribution is zero, $b_{3}=0$, for the following effective number of families

$$
n=\frac{34}{3} C_{A}^{2} /\left(\frac{20}{3} C_{A}+4 C_{F}\right) T_{F}
$$

In the case of the $S U(2)$ group, the effective families number is $n=136 / 49 \simeq 2.78 \simeq 3$. 


\subsection{Next to the minimal renormdinamics of QCD}

We have seen that motion equation of the minimal renormdynamics of the one charge QCD is

$$
\begin{aligned}
& \dot{x}=-b_{2} x^{2}-b_{3} x^{3}, \\
& b_{2}=11-\frac{2}{3} n, b_{3}=2\left(51-\frac{19}{3} n\right), x=\frac{\alpha_{s}}{4 \pi}=\left(\frac{g}{4 \pi}\right)^{2},
\end{aligned}
$$

when we ask that IR fixed point is $\alpha_{s}=2, a=c=1 / 2 \pi$, we take nontrivial next term in the motion equation,

$$
\begin{aligned}
& \dot{x}=\beta_{2} x^{2}+\beta_{3} x^{3}+\beta_{4} x^{4}=\beta_{2} x^{2}\left(1+c_{1} x+c_{2} x^{2}\right), \\
& 1+c_{1} c+c_{2} c^{2}=0 \Rightarrow c_{2}=\beta_{4} / \beta_{2}=-\left(1+c \beta_{3} / \beta_{2}\right) / c^{2}=-\left(1+c b_{3} / b_{2}\right) / c^{2}, \\
& b_{2}=11-\frac{2}{3} n, b_{3}=2\left(51-\frac{19}{3} n\right), x=\frac{\alpha_{s}}{4 \pi}=\left(\frac{g}{4 \pi}\right)^{2},
\end{aligned}
$$

When $\beta_{3}=0$,

$$
\dot{x}=\beta_{2} x^{2}\left(1-x^{2} / c^{2}\right)
$$

Note that due to

$$
c_{2} x^{2}+c_{1} x+1=c_{2}\left(x-x_{1}\right)\left(x-x_{2}\right), c_{2} x_{1} x_{1}=1, x_{2}=1 / c_{2} x_{1}=-\frac{2 \pi}{1+2 \pi b_{3} / b_{2}}
$$

is the UV fixed point. The negative value of the $\alpha_{s}$ indicates instability at higher energies, but before the coupling constant reach that value from low energy evolution it will stop at at the trivial zero. So, from higher energy, in heavy ion collision e.g. we expect the instable phase of QCD. Similar consideration takes place for QED, with IR fixed point of $\alpha=\alpha_{*}=1 / 137.036 \ldots$ Now we should include next term in the $\beta$-function

$$
\dot{x}=\beta_{2} x^{2}+\beta_{3} x^{3}+\beta_{4} x^{4}+\beta_{5} x^{5}=\beta_{2} x^{2}\left(1+c_{1} x+c_{2} x^{2}+c_{3} x^{3}\right),
$$

with UV fixed point at $\alpha=2$

\subsection{Next to the critical renormdynamics}

We have seen, that one charge renormdynamic motion equation of QCD

$$
\dot{a}=\beta(a)=\beta_{1} a+\beta_{2} a^{2}+\beta_{3} a^{3}+\beta_{4} a^{4}+\ldots
$$

is critical, $\beta_{1}=0$ in four dimensional space-time. When not only $\beta_{1}=0$ but also $\beta_{2}=0$ we have the next to the critical renormdynamics. This case, by reparametrization

$$
a=f(A)=A+f_{2} A^{2}+f_{3} A^{3}+\ldots
$$

we obtain the following minimal form of renormdynamics

$$
\dot{A}=b_{3} A^{3}+b_{5} A^{5}, b_{3}=\beta_{3}, b_{5}=\beta_{5}+2 f_{2} \beta_{4}+f_{2}^{2} \beta_{3}
$$

Indeed,

$$
b_{4}=\beta_{4}+f_{2} \beta_{3}, b_{4}=0 \Rightarrow f_{2}=-\beta_{4} / \beta_{3} \Rightarrow b_{5}=\beta_{5}-\beta_{4}^{2} / \beta_{3},
$$




$$
b_{5}=0 \Rightarrow \beta_{3} \beta_{5}=\beta_{4}^{2}
$$

This case we will call the second critical case with the solution

$$
A(t)=\frac{A_{0}}{\sqrt{1-2 b_{3} A_{0} t}}
$$

Beyond the second critical case, when $b_{5} \neq 0$, the motion equation of renormdynamics reduce to first critical form

$$
\dot{x}=k_{2} x^{2}+k_{3} x^{3}, x=A^{2}, k_{2}=2 b_{3}, k_{3}=2 b_{5} .
$$

General one charge-dimensional renormdynamic motion equation is convenient put in form

$$
\begin{gathered}
\dot{a}=\beta_{m} a^{m}\left(1+c_{1} a+c_{2} a^{2}+\ldots\right)=\beta_{m} a^{m} c(a), m \geq 1 \\
\dot{a}=\beta_{1}\left(A+f_{2} A^{2}+f_{3} A^{3}+\ldots\right)+\beta_{2}\left(A+f_{2} A^{2}+f_{3} A^{3}+\ldots\right)^{2} \\
+\beta_{3}\left(A+f_{2} A^{2}+f_{3} A^{3}+\ldots\right)^{3}+\beta_{4}\left(A+f_{2} A^{2}+\ldots\right)^{4}+\beta_{5}(A+\ldots)^{5}+\ldots
\end{gathered}
$$

\subsection{QED three loop $\beta$-function}

The renormdymamic $\beta$-function is given up to third order in $\alpha$ by De Rafael and Rosner [?]

For the electron-photon QED it is known 5-loop $\beta$-function [Kataev, Larin, 2012]. We will consider only three-loop approximation

$$
\begin{aligned}
& \mu^{2} \frac{d a}{d \mu^{2}}=\dot{a}=\beta(a)=a^{2}\left(\beta_{0}+\beta_{1} a^{a}+\beta_{2} a^{2}\right), \\
& a=\left(\frac{e}{2 \pi}\right)^{2}, \beta_{0}=\frac{1}{3}, \beta_{1}=\frac{1}{4}, \beta_{2}=-\frac{31}{288},
\end{aligned}
$$

\subsection{Why theoretical and experimental values of $\alpha_{\pi N}$ differ}

Qualitatively the difference between our theoretical value of $\alpha_{\pi N}=13$ and the values extracted from experimental date $\alpha_{e x \pi N} \geq 14$ can be explained by following examples

$$
\begin{aligned}
& \text { 1. } \alpha_{e x \pi N}=\alpha_{\pi N}\left(1+x+x^{2}+\ldots\right)=\alpha_{\pi N} /(1-x) \\
& =\frac{13}{12} \alpha_{\pi N}=\frac{13}{12} 13=14.08, x=|13|_{p}=1 / 13 \\
& \text { 2. } \alpha_{e x \pi N}=\alpha_{\pi N}\left(1+x+x^{2} / 2 !+\ldots\right)=\alpha_{\pi N} e^{x}=13 e^{1 / 13}=14.04
\end{aligned}
$$

\subsection{Exact Renormdynamics}

The perturbative $\beta$-function is known exactly in a number of supersymmetric theories and in the 't Hooft renormalization scheme in the $\varphi_{4}^{4}$ model. 


\subsection{Perturbatively Exact Renormdynamics}

From renormdynamics to exect dynamics. In a number of instances, the perturbative renormalization group $\beta$-function is known exactly. In $N=4$ supersymmetric Yang-Mills (SYM) theory, an $\beta$-function vanishes [MandeIstam, 1983], [Howe,Stelle,Townsend, 1983]. In $N=2$ SYM theory, the $\beta$-function is exact at one-loop order when minimal subtraction (MS) is used [Howe,Stelle,West, 1983], [Koh,Rajpoo, 1983]. In $N=1$ SYM theory, the all-orders expression for the $\beta$-function can be determined through instanton calculus [Novikov et a [983] or by considering the multiplet structure of anomalies [Dons, 1983], [Elias, 2001], though such an expression differs from the perburbative result derived using MS [Dack,Jones,North, 1997], [Dack,Jones,Pickering, 1998]. Although models such as the $\varphi_{4}^{4}$ scalar theory and Yang-Mills (YM) theory have all-orders contributions to the $\beta$-function in the MS scheme, one can nevertheless perform in principle a finite renormalization at each order of perturbation theory so as to have contributions to the $\beta$-function vanish beyond two-loop order and to have anomalous dimensions as entirely one-loop effects.

\subsection{Infrared fixed points of the renormdynamics}

QCD ultraviolet fixed point is at zero. The value of the infrared fixed point depends on the definition of $\alpha_{s}$, but the scale at which we have infrared fixed point is universal. Indeed, we have

$$
\begin{aligned}
& a=f(A), \\
& \dot{a}=\beta_{a}=f^{\prime}(A) \dot{A}, \\
& 0=\dot{a}=\beta_{a} \Leftrightarrow \dot{A}=\beta_{A}=0 .
\end{aligned}
$$

By different parametrization, we will have different infrared fixed values. The nearest prime number to zero is the fist prime number 2. Next one is 3 . There are parameterizations corresponding to these values of infrared fixed points which we prefer.

\subsection{Strong coupling models of QCD}

We know that old four-fermion week interaction of Fermi is low energy effective theory of the $U(1) \cdot S U(2)$ part of SM. The value of the Fermi constant

$$
G \sim 10^{-5} m_{p}^{-2} \sim m_{W}^{-2}, m_{W} \sim 10^{2} \mathrm{GeV}
$$

Photon (in vacuum) and gluon, in perturbation theory approximation, are massless, so we have not local effective theory like Fermi interaction. If we suppose that gluon at low energy become massive, we will have Fermi type effective theory,

$$
L_{Q C D}=\bar{q}\left(\gamma D-m_{q}\right) q-G j j-A_{\mu}^{a} J_{\mu}^{a}, j_{\mu}^{a}=\bar{q} \gamma_{\mu} t^{a} q
$$

where $J$ is external source of the gluon field.

\subsection{Exact renormdynamics of QCD}

In a sense QCD in the critical dimension 4 is simple than beyond, because dynamical dimension is an other coupling constant. So, let us start in critical dimension. We have seen that in one dimensional renormdynamics, the coupling function is monoton function of the scale and connects 
UV and IR fixed points. UV fixed point is zero, IR fixed point is $\alpha_{s}=2$. Now we will formalize (formulize, if you like) this statement in the exact renormdynamic beta-function. We know that first two term of the beta-function is reparametrization invariant. There are parametrizations, were we have positive third term and zero higher terms,

$$
\begin{aligned}
& \dot{a}=-a_{2} a^{2}-a_{3} a^{3}+a_{4} a^{4}=a_{4} a^{2}\left(a^{2}-2 b a-c\right), b=\frac{a_{3}}{2 a_{4}}>0, c=\frac{a_{2}}{a_{4}}>0, \\
& \dot{a}=\frac{d a}{d t}=\frac{\mu^{2} d}{d \mu^{2}} a, a_{2}=11-\frac{2}{3} n_{f}, a_{3}=102-\frac{38}{3} n_{f},
\end{aligned}
$$

where $n_{f}$ is the number of quarks with masses less than the energy scale $\mu$. For IR fixed point we have

$$
4 \pi a=\alpha_{I R}=2=b+\sqrt{b^{2}+c} \Rightarrow a_{4}=\frac{a_{3}}{2}+\frac{a_{2}}{4}
$$

Beyond critical dimension in one loop approximation

$$
\dot{a}=(D-4) a-k a^{2}, \dot{a}=\frac{d a}{d t}=\frac{\mu^{2} d}{d \mu^{2}} a
$$

For $D>4$, we have UV fixed point at $(D-4) / k$ and IR fixed point at 0 . For small $\varepsilon=D-4$ this is plausible picture: at small distances we will have small deviation from scaling; at big distances we have not colored particles. In this case colorless fractionally charged quarks with finite mass exist. If the quarks are only valence and there masses depend on the coupling constant as $\sim \alpha^{-n}$, as nonperturbative solutions of nonlinear equations, the masses rise with distance and quarks are unobservable on large scales. For $D<4$, we have asymptotic freedom on small scales and IR fixed point,

$$
2=\alpha_{I R}=\frac{k}{D-4} \Rightarrow k=2(D-4)<0
$$

\subsection{Toward the Finite Unified Field Theory}

The reduction of the dimensionless couplings in $\mathrm{N}=1$ GUTs is achieved by searching for integrals of motion-renormdynamic invariant (RDI) relations among them holding beyond the unification scale. Finiteness results from the fact that there exist RDI relations among dimensional couplings that guarantee the vanishing of all beta-functions in certain $\mathrm{N}=1$ GUTs even to all orders. Developments in the soft supersymmetry breaking sector of $\mathrm{N}=1$ GUTs and FUTs lead to exact RDI relations, i.e. reduction of couplings, in this dimensionful sector of the theory, too. Based on the above theoretical framework phenomenologically consistent FUTs have been constructed. The main goal expected from a unified description of interactions by the particle physics community is to understand the present day large number of free parameters of the Standard Model (SM) in terms of a few fundamental ones. In other words, to achieve reduction of couplings at a more fundamental level.

\subsection{Noncommutative QFT}

QFT require regularization and the conventional nonperturbative regularization is lattice regularization. It has been extensively studied for forty years. It fails to preserve space-time symmetries 
of quantum fields. It also has problems in dealing with topological subtleties like instantons, and can deal with index theory and axial anomaly only approximately. Instead fuzzy (noncommutative) physics does not have these problems. A related positive feature of fuzzy physics, is its ability to deal with supersymmetry(SUSY) in a precise manner [Grosse,Klimcik,Presnajde, 1997],

[Balachandra,Kurkcuoglu,Rojas,2002]. Fuzzy SUSY models are also finite-dimensional matrix models amenable to numerical work, so this is another reason for our attraction to this field.

\subsection{Scalar quarks and MSSM}

One of the most important tasks at the LHC is to search for physics effects beyond the Standard Model (SM), where the Minimal Supersymmetric Standard Model (MSSM) [Nilles, 1984] is one of the leading candidates.

Typically, strongly bound solutions of the Bethe-Salpeter equation exist only when the coupling constant is on the order of or greater than unity.

Three times in the past 150 years particles that were - or are currently - thought to be elementary could be organized into families: (1) In the 1860's Meyer and Mendelev arranged the atoms into the periodic table that consists of 18 families. The existence of families was ultimately explained by the realization that atoms are composite. (2) Almost 100 years later two of the thenfundamental particles, the neutron and proton - as well as many other baryons and mesons-were placed into $\mathrm{SU}(3)$ multiplets with the ultimate result that the neutron and proton were no longer viewed as being fundamental but instead were comprised of quarks. (3) By the mid 1970's many physicists realized that the charged leptons, the neutrinos, the positively charged quarks and the negatively charged quarks constitute four families. Today the three charged families are known to have three members each, and the neutrino family almost certainly has three members as well [3]. Because the existence of families of "fundamental" particles has twice been explained by the realization that the particles are actually composite, although speculative, as is any physics beyond the standard model, the most conservative approach to explain the existence of the lepton and quark families likely is to assume that the particles are composite. In the 1970's the hypothetical constituents of quarks and leptons were given the name "preons" [Pati,Salam,1974].

\subsection{AdS/QCD as nice phenomenology}

For AdS/CFT connection it is necessary that the QFT part of the connection is a conformal field. In the case of the QCD we do not know for sure the scale at which we are at the IR fixed point beyond which we are in the hadronic phase. I suggested that at that point $\alpha_{s}=2$.

\subsection{Graphene}

Graphene is a one-atom-thick allotrope of carbon, with unusual two-dimensional Dirac-like electronic excitations. The Dirac electrons can be controlled by application of external electric and magnetic fields, or by altering sample geometry and/or topology. The Dirac electrons behave in unusual ways in tunneling, confinement, and the integer quantum Hall effect. The electronic properties of graphene stacks are discussed and vary with stacking order and number of layers. Edge (surface) states in graphene depend on the edge termination (zigzag or armchair) and affect the physical properties of nanoribbons. Different types of disorder modify the Dirac equation leading 
to unusual spectroscopic and transport properties. There are different effects of electron-electron and electron-phonon interactions in single layer and multilayer graphene [Castro Neto et al, 2009].

Carbon is the materia prima for life and the basis of all organic chemistry. Because of the flexibility of its bonding, carbon-based systems show an unlimited number of different structures with an equally large variety of physical properties.

The effective field model of graphene (EFG) monolayer without the Coulomb interactions is a good approximation to the original tight - binding model. The EFG model operates with the continuum Dirac field living in the graphene sheet.

The principle feature of graphene is that the quasi-particle excitations satisfy the Dirac equation, where the speed of light $c$ is replaced by the so-called Fermi velocity $v_{F} \simeq c / 300$. Therefore, the quantum field theory methods are very useful in the physics of graphene. By applying these methods, one can explain anomalous Hall Effect in graphene, the universal optical absorption rate, the Faraday effect, and predict the Casimir interaction of graphene, and do much more (see, e.g. [Fialkovsky, vassilevich, 2011]).

The Dirac model for quasi-particles in graphene was elaborated in full around 1984 - twenty years before actual discovery of graphene. However, its basic properties, like the linearity of the spectrum, etc., were well known and widely used much earlier due to the 1947 paper by Wallace. The purpose of most of the works of the time was to describe graphite rather than graphene (see review [Castro Neto et al, 2009]).

Note that, effective fine structure constant in graphene is $\alpha_{g} \simeq 300 \alpha=2.19$ We will take $\alpha_{g}=2$ and consider p-adic perturbation theory for graphene. Corresponding effective light speed is $c_{g}=274$, which is two orders more precise prediction-proposal than $c_{g} \simeq 300$.

\subsection{Conformal Field Theories, Mellin Amplitudes}

Mellin amplitudes are an alternative representation of conformal correlation functions that are analogous to scattering amplitudes. The Operator Product Expansion (OPE) leads to the factorization of the residues of the poles of Mellin amplitudes [Gonalves et al, 2014]. Convergent OPE is a basic property of a Conformal Field Theory (CFT). This means that we can replace the product of $\mathrm{m}$ local operators (inside a correlation function) by an infinite sum of local operators

$$
Q_{1}\left(x_{1}\right) Q_{2}\left(x_{2}\right) \ldots Q_{m}\left(x_{m}\right)=\sum_{p} A_{\mu_{1} \mu_{2}, \ldots, \mu_{k}}^{1,2, \ldots, m ; p}\left(x_{1}, \ldots x_{m}, y, \partial_{y}\right) Q_{p}^{\mu_{1} \mu_{2}, \ldots, \mu_{k}}(y)
$$

where $\mathrm{p}$ runs over all primary local operators. This sum converges inside a M-point correlation function if there is a sphere centred at $\mathrm{y}$ that contains all points $x_{1}, \ldots, x_{m}$ and does not contain any of the other $M-m$ points.

25.109 Mittag-Leffler Functions $E_{\alpha}(z), E_{\alpha, \beta}(z)$

Mittag-Leffler function $E_{\alpha}(z)$ is defined over the entire complex plane by

$$
E_{\alpha}(z)=\sum_{n \geq 0} \frac{z^{n}}{\Gamma(\alpha n+1)}, \alpha>0, z \in \mathbf{C}
$$


Note that $E_{1}(z)=\exp (z)$, so, it is extension-deformation of usual $\exp (z)$. The two parameter deformation also considered

$$
E_{\alpha, \beta}(z)=\sum_{n \geq 0} \frac{z^{n}}{\Gamma(\alpha n+\beta)}=\frac{\Gamma(\delta+1)}{\Gamma(\alpha \delta+\beta)} \exp (z), \alpha, \beta>0, z \in \mathbf{C}
$$

Note that $E_{\alpha, 1}(z)=E_{\alpha}(z)$. Using our definition of the fractal calculus

$$
D^{\alpha} f(z)=z^{-\alpha} \frac{\Gamma(\delta+1)}{\Gamma(\delta+1-\alpha)} f(z), \delta=z \partial_{z}
$$

we obtain

$$
E_{1, \beta}(z)=z^{1-\beta} D^{1-\beta} \exp (z)
$$

Note that $E_{1,1}(z)=\exp (z)$

\subsection{Higher Spin Gauge Fields}

In the standard model of particle physics, interaction between matter field particles, quarks and leptons, devised on massless vector gauge fields, gluons, photons, $\mathrm{W}$ and $\mathrm{Z}$ bosons-massive due to scalar higgs fields. Einstein-Hilbert theory of gravitation, is based on the tensor gauge field, local metric of space-time $g_{\mu v}(x)$. Existence of a vector massless gauge field is connected with existence of corresponding conserved vector current. E.g. in electrodynamics motion equation for photons is

$$
\partial_{\mu} F^{\mu v}=J^{v}, \partial_{\mu} J^{\mu}=0
$$

Existence of the interacting Higher Spin Gauge Fields (HSGF) are connecting with the existence of conserved tensors. In the case of the gravity, with the energy-momentum tensor. The manifolds with nontrivial conserved tensor fields are candidates of the backgrounds for HSGF.

\subsection{Mass Gap Problem in Yang-Mills Field Theory and QCD}

Yang-Mills theory is the name of a relativistic quantum field theory exhibiting local gauge invariance (i.e., gauge invariance of the second kind). It describes a quantized field corresponding to the connection 1-form with values in the Lie algebra of a Lie group, G, called the gauge group; (in $\mathrm{QED}$, this field is the vector potential, and $\mathrm{G}=\mathrm{U}(1)$ ). There may be other quantum fields in the theory, such as matter fields (quantized sections of G-vector bundles). One important problem is to show that Yang-Mills theories compatible with relativistic invariance (i.e., without ultraviolet cutoffs) exist in four space-time dimensions. If this can be shown then the joint spectrum of the energy-momentum operator forms a Lorentz-invariant subset of the forward light cone in fourdimensional momentum space. If the theory has a ground state (called "vacuum" in quantum field theory) then the origin, 0 , is an eigenvalue of the energy-momentum operator. The mass gap problem is the problem to show that, above the eigenvalue 0 of the energy operator (the Hamiltonian of the theory) the spectrum of the Hamiltonian exhibits a gap, i.e., excitations of energy arbitrarily close to 0 do not exist. One can formulate this property in terms of the mass operator,

$$
M=\sqrt{P_{0}^{2}-P_{n}^{2}}
$$


and say that one would like to show that the operator $M$ has a spectral gap above the eigenvalue 0 (corresponding to the vacuum).

In four space-time dimensions, this problem remains open from the point of view of rigorous mathematics; (in spite of heroic efforts and great results due to Tadeusz Balaban, and some others). This problem is one of the "Clay one-million dollars problems". It is presumably a very difficult problem, and there are no clear ideas of how to go about solving it.One should add that, from a more heuristic physics point of view, there are good reasons to think that the problem has a positive solution. In this connection, insights gained from "lattice gauge theory" - the study of which has originally been initiated by the late Ken Wilson - and numerical simulations of lattice gauge theories have played a very useful role.

\subsection{Social profit of big collaborations}

Nowadays there are several big collaborations in science, e.g. LHC. Scientific value of LHC depends on three components, the highest quality of accelerator, highest quality of detectors and distributed data processing. The first two components need good mathematical and physical modeling. Third component and the collaboration as a social structure are not anther the control by scientific methods and corresponding modeling.

By definition, scientific collaborations (SC) have a main scientific aim: to obtain answer on the important scientific question(s) and maybe gain extra scientific bonus: new important questions and discoveries.

$\mathrm{SC}$ is more open information system than e.g. finance or military systems. So, it is possible to describe and optimize SC by scientific methods. Profit from scientific modeling of SC maybe also for other information systems and social structures.

\section{Appendixes}

\subsection{Mathematica}

The following short program uses the command Plot to graph the functions $x^{2}, x^{3}$, and $x^{4}$ from $x=0$ to $\mathrm{x}=1$. The graphs are stored in the graphic objects $g r 1, g r 2$, and $g r 3$. Finally, the three graphs are shown together by using the Mathematica command Show,

$$
\begin{aligned}
& \operatorname{gr} 1=P \operatorname{Plot}\left[x^{2},\{x, 0,1\}\right] ; \\
& \operatorname{gr} 2=\operatorname{Plot}\left[x^{3},\{x, 0,1\}\right] ; \\
& \operatorname{gr} 3=\operatorname{Plot}\left[x^{4},\{x, 0,1\}\right] ; \\
& \text { Show }[\{\operatorname{gr} 1, \operatorname{gr} 2, \operatorname{gr} 3\}]
\end{aligned}
$$

Mathematica can also solve systems of differential equations as seen in the following example

$$
\begin{aligned}
& \text { sol }=N D S o l v e\left[\left\{x^{\prime}[t]==(\operatorname{Exp}[y[t]]-\operatorname{Exp}[z[t]]), y^{\prime}[t]==(\operatorname{Exp}[z[t]]-\operatorname{Exp}[x[t]]),\right.\right. \\
& \left.z^{\prime}[t]==(\operatorname{Exp}[x[t]]-\operatorname{Exp}[y[t]]), x[0]==y[0]==1\right\}, \\
& \{x, y\},\{t, 20\}] x[0]==3, y[0]==4, z[0]==4\},\{x, y, z\},\{t, 10\}] ; \\
& \text { Plot }[\{x[t] / . \operatorname{sol}, y[t] / . s o l, z[t] / . s o l\},\{t, 0,3\}]
\end{aligned}
$$

with appropriate initial conditions. The command Plot is again used to graph the solutions $x(t), y(t)$ and $z(t)$. 


\subsection{Relativistic particle propagator}

$$
\begin{aligned}
& G(x-y, D, m)=\frac{1}{(2 \pi)^{D}} \int d^{D} k \frac{e^{i k(x-y)}}{m^{2}+k^{2}}=\frac{1}{(4 \pi)^{D / 2}} \int_{0}^{\infty} \frac{d \alpha}{\alpha^{D / 2}} e^{-m^{2} \alpha-\frac{(x-y)^{2}}{4 \alpha}}, \\
& G(x, D, 0)=\frac{\Gamma(D / 2-1)}{4 \pi^{D / 2} x^{D-2}}, \\
& G(x, 4,0)=\frac{1}{4 \pi^{2} x^{2}}, G(x, 3,0)=\frac{1}{4 \pi x}, G(x, 1,0)=\frac{x}{2}
\end{aligned}
$$

In the case of $D=2+2 \varepsilon$,

$$
G(x, 2+2 \varepsilon, 0)=\frac{\Gamma(\varepsilon)}{4 \pi^{1+\varepsilon} x^{\varepsilon}}=\frac{1}{4 \pi}\left(\frac{1}{\varepsilon}-\gamma-\ln \pi x\right)=-\frac{1}{4 \pi} \ln M x+O(1 / \varepsilon)
$$

\subsection{Relativistic kinematics}

For the elastic two particle scattering

$$
a\left(p_{1}\right)+b\left(p_{2}\right) \rightarrow a\left(p_{1}^{\prime}\right)+b\left(p_{2}^{\prime}\right),
$$

the Mandelstam variables $s$ and $t$ are

$$
\begin{aligned}
& s=\left(p_{1}+p_{2}\right)^{2}=E_{c m}^{2}=4 E^{2}=4 m^{2}+4 p^{2}>0 \\
& t=\left(p_{1}-p_{1}^{\prime}\right)^{2}=q^{2}=2 m^{2}-2\left(m^{2}+p^{2}-2 p^{2} \cos \theta\right) \\
& =-4 p^{2} \sin ^{2} \theta / 2 \simeq p^{2} \theta^{2} \simeq p^{2} \sin ^{2} \theta=-k^{2}<0 \\
& u=\left(p_{1}-p_{2}^{\prime}\right)^{2}=2 m^{2}-2\left(m^{2}+p^{2}-2 p^{2} \cos (\pi-\theta)\right) \\
& =-4 p^{2} \cos ^{2} \theta / 2 \\
& s+t+u=4 m^{2} .
\end{aligned}
$$

where, for simplicity, we take equal mass particles; $\mathrm{p}$ is the length of the momentum in the CM system and $k$ is the transverse momentum of the particle, $k=p_{1}^{\prime} \sin \theta$.

For the Reggeon kinematics, $s>>m^{2},-t / s<<1$, the scattering is effectively described by exchange of the Reggeon quasi particle with the angular momentum as a function of the transfer momentum, $t=q^{2}$

$$
J=\alpha\left(q^{2}\right)=\alpha(0)+\alpha^{\prime} q^{2}+\ldots \simeq 1-\alpha^{\prime} k^{2},
$$

so, we have non-relativistic $1+2$ dimensional analog system with energy, momentum and mass

$$
E=\frac{k^{2}}{2 M}=1-J, M=1 / 2 \alpha^{\prime} .
$$

Rapidity is defined as

$$
\begin{aligned}
& Y=\frac{1}{2} \ln \frac{E+p_{\|}}{E-p_{\|}}=\frac{1}{2} \ln \frac{4 E^{2}-2 E\left(E-p_{\|}\right)-E_{\perp}^{2}}{E_{\perp}^{2}} \\
& \simeq \ln \frac{\sqrt{s}}{E_{\perp}}, E_{\perp}^{2}=m^{2}+p_{\perp}^{2}
\end{aligned}
$$




\subsection{Experiments at high-energy colliders}

In particle colliders, collisions actually take place between beams containing large number of particles. If two beams collide head-on, the number of collisions leading to a final state with particular characteristics (type of particles, their momenta, etc.) should be proportional to the number of particles in each beam, $N_{a}$ and $N_{b}$, and inversely proportional to the beams cross-sectional area $A$. The coefficient of proportionality is the scattering cross section for this particular final state:

$$
N=\sigma \frac{N_{a} N_{b}}{A},
$$

where the coefficient of proportionality $\sigma$ is the scattering cross section for this particular final state.

If beams collide at a frequency $f \mathrm{~Hz}$, the rate $R$, number of events of a particular kind recorded per second, can be written as

$$
R=N f=\sigma L
$$

where

$$
L=f \frac{N_{a} N_{b}}{A}
$$

is the instantaneous luminosity. The rate $R$ is measured directly by experimentalists; $L$ (together with $E_{c m}$ ) contains all the information about the accelerator needed to analyze the experiment. Experimental collaborations monitor and record $L$, as a function of time. The experimentally measured value of the cross section can then be compared with the theoretically expected cross section. Note that, $L$ is higher for higher $f$ and/or lower $A$. The values of $f$ and $A$ are connected to the bunch size, length and radius.

A unit typically used in experimental nuclear and particle physics is 1 barn $=10^{-24} \mathrm{~cm}^{2}$. In theory units, $c=\hbar=1$, the natural unit for cross section is $\mathrm{GeV}^{-2}$; the conversion factor is

$$
\begin{aligned}
& 1 b n=2568 \mathrm{GeV}^{-2}, \\
& 1 \mathrm{GeV}^{-2}=3.89410^{-4} b n .
\end{aligned}
$$

To get a very rough estimate of cross sections expected in particle physics experiments, we can use dimensional analysis: away from thresholds and resonances, the only energy scale in a collision of two massless particles is $E_{c m}$, and we should expect the (total) scattering cross section to behave roughly as

$$
\sigma \sim E_{c m}^{-2}
$$

A similar result is obtained by replacing the colliding particles with a balls of diameter equal to their Compton wavelength $d \sim 1 / E$, and taking their geometric cross section as an estimate. The geometric cross section also coincides with the upper bound on the total inelastic cross section (assuming $s$-wave scattering) from unitarity considerations.

The cross sections for specific processes are typically lower, than this bound: For example, the $e^{+} e^{-} \rightarrow Z$ cross section on resonance $\left(\sqrt{s}=M_{Z}\right)$ is about $40 \mathrm{nb}$, compared to $\sigma_{\text {geom }}=1 / M_{Z}^{2} \simeq 47 \mathrm{nb}$. 
The decrease of cross sections with energy has an important implication for accelerator design: Colliders operating at higher center-of-mass energies must also have higher luminosity, adding to the technical challenges of expanding the high-energy frontier.

The main formula for evaluating the cross section and kinematic distributions for a $2 \rightarrow N$ scattering process is

$$
d \sigma=\frac{1}{2 s} \prod_{n=1}^{N} \frac{d^{3} p_{n}}{(2 \pi)^{3} 2 E_{n}} \cdot(2 \pi)^{4} \delta^{4}\left(p_{a}+p_{b}-\Sigma p_{n}\right) \cdot \mid M\left(p_{a}, p_{b} ;\left.\left\{p_{n}\right\}\right|^{2},\right.
$$

where $M$ is the invariant matrix element, and $p_{n}=\left(E_{n}, p_{n}\right)$ are the 4-momenta of the final-state particles. $M$ contains all information specific for the process under consideration (such as coupling constant dependence, etc.), whereas all other ingredients are simply kinematic factors common for any $2 \rightarrow N$ process. Eq. (26.55) is written in the center of mass frame of the colliding particles, but it is in fact invariant under boosts parallel to the collision axis. This feature is important when hadron collisions are considered. If the colliding beams are unpolarized, one needs to average the quantity $|M|^{2} \mid$ over all possible initial-state polarizations. If the beams are polarized , an appropriately weighted average should be computed instead. In addition, if the final-state particles have spin, $|M|^{2}$ should typically be summed over all possible spin states, since no collider detector is capable of detecting spins of individual particles. (Exception occurs when the final-state particles decay promptly, in which case the angular distribution of their decay products may carry information about their polarization state.) The appropriately averaged and/or summed scattering amplitude will be denoted by $\overline{|M|^{2}}$.

The number of independent kinematic variables in a $2 \rightarrow N$ process is $3 N-4$. In practice, the initial state is always symmetric under rotation around the collision axis, and no physical observable can depend on the overall azimuthal coordinate, leaving $3 N-5$ physical variables.

The simplest case, most commonly encountered in practice, is $2 \rightarrow 2$ scattering. The only observable not constrained by energy and momentum conservation is the scattering angle $\theta$, which by convention is defined as the angle between the 3-momenta of particles a and 1 . The differential cross section is given by

$$
\frac{d \sigma}{d(\cos \theta)}=\frac{1}{16 \pi} \frac{\left|p_{1}\right|}{s^{3 / 2}} \cdot \overline{|M|^{2}}, \sqrt{s}>m_{1}+m_{2}
$$

where

$$
\left|p_{1}\right|=\sqrt{\frac{\left(s-m_{1}^{2}-m_{2}^{2}\right)^{2}-4 m_{1}^{2} m_{2}^{2}}{4 s}} .
$$

In the case of equal masses in the final state, $m_{1}=m_{2}=m$, this formula further simplifies to

$$
\frac{d \sigma}{d(\cos \theta)}=\frac{1}{32 \pi s} \sqrt{1-\frac{4 m^{2}}{s}} \cdot \overline{|M|^{2}}, \sqrt{s}>2 m \text {. }
$$

where the square-root factor is simply the velocity of the final-state particles (in units of $c$ ). The quantity $\overline{|M|^{2}}$ is often expressed in terms of the Mandelstam variables, $s$ and $t$.

The main advantage of using Mandelstam variables comes in applications of crossing symmetry to relate processes such as, for example, electron-positron annihilation $e^{+} e^{-} \rightarrow \gamma \gamma$ and Compton scattering $e^{-} \gamma \rightarrow e^{-} \gamma$. They are also convenient for analyzing hadron collisions, being invariant under boosts connecting the parton and lab reference frames. 


\subsection{Inverse scattering transform (IST)}

IST is a non-linear analogue, and in some sense generalization, of the Fourier transform, which is applied to solve many linear partial differential equations. IST is a method for solving some nonlinear partial differential equations. The name "inverse scattering method" comes from the key idea of recovering the time evolution of a potential from the time evolution of its scattering data: inverse scattering refers to the problem of recovering a potential from its scattering matrix, as opposed to the direct scattering problem of finding the scattering matrix from the potential. A characteristic of solutions obtained by the IST is the existence of solitons, solutions resembling both particles and waves, which have no analogue for linear partial differential equations. The term "soliton" arises from non-linear optics. The IST can be written as a Riemann-Hilbert factorization problem. This formulation can be generalized to differential operators of order greater than 2 and also to periodic potentials.

\section{References}

[Aad et al. ATLAS Collaboration, 2012] G. Aad et al. [ATLAS Collaboration], Phys. Lett. B 716, 1 (2012)

[Abrikosov, 1957] Abrikosov, A. A. (1957) On the Magnetic properties of superconductors of the second group, Sov.Phys.JETP 5:1174-1182 and Zh.Eksp.Teor.Fiz.32:1442-1452.

[Ackermann et al, 2013] Ackermann, M.; Ajello, M.; Allafort, A.; Baldini, L.; Ballet, J.; Barbiellini, G.; Baring, M. G.; Bastieri, D. et al. (2013-02-15). Detection of the Characteristic Pion-Decay Signature in Supernova Remnants. Science (American Association for the Advancement of Science) 339 (6424): 807-811.

[Adare et al, 2007] A. Adare et al. (PHENIX Collaboration), Phys. Rev. Lett. 98, 162301 (2007).

[Adler, 1965] S. L. Adler, Phys. Rev. 140 (1965) B736.

[Adler, Dashen, 1965] S.L. Adler, R. Dashen, Current Algebra and Applications to Particle Physics, Benjamin, New York 1965.

[Aharony et al, 2000] O. Aharony, S. S. Gubser, J. M. Maldacena, H. Ooguri and Y. Oz, Large N field theories, string theory and gravity, Phys. Rept. 323 (2000) 183, hep-th/9905111.

[ALICE, 2010] K. Aamodt et al. [ALICE collaboration] Eur. Phys. J. C65 (2010) 111 [arXiv: 1004.3034 ], [arXiv: 1004.3514$]$.

[Al Khawaja,Stoof,2001] Al Khawaja, Usama; Stoof, Henk (2001). "Skyrmions in a ferromagnetic BoseÛEinstein condensate". Nature 411 (6840): 918 Ü20.

[Anselmino,Ioffe,Leader,1989] M. Anselmino, B.L. Ioffe and E. Leader, Sov. J. Nucl. 49, 136, (1989)

[Aref, 1983] H. Aref, Ann. Rev. Fluid Mech. 15 (1983) 345.

[Arnold, 1978] V.I. Arnold, Mathematical Methods of Classical Mechanics, Springer, New York 1978.

[Axenides, Floratos, Perivolaropoulos, 2000] M.Axenides,E.G.Floratos,L.Perivolaropoulos, Dynamical Effects of the Cosmological Constant (astro-ph/0004080) Modern Physics Letters A 15, 1541-1550

[Bagger, Lambert, 2007] J. Bagger, N. Lambert, Modeling multiple M2's, Phys. Rev. D 75 (2007) 045020; [arXiv:hep-th/0611108].

[Bae et al. 2000-2008] D. Lopez-Val and J. Sola, Phys. Lett. B 702, 246 (2011) [arXiv:1106.3226 [hep-ph]]; P. Niezurawski, A. F. Zarnecki and M. Krawczyk, eConf C 050318, 0112 (2005) [hep-ph/0507006]; M. M. Muhlleitner et al., Phys. Lett. B 508, 311 (2001) [hep-ph/0101083]. S. Bae, B. Chung and P. Ko, Eur. Phys. J. C 54, 601 (2008) [hep-ph/0205212]; S. Y. Choi and J. S. Lee, Phys. Rev. D 62, 036005 (2000) [hep-ph/9912330]. 
[Bakas et al,2007] I. Bakas, D. Orlando and P.M. Petropoulos, Ricci flows and expansion in axiondilaton cosmology, JHEP 0701 (2007) 040 [arXiv:hep-th/0610281].

[Balachandra,Kurkcuoglu,Rojas,2002] A. P. Balachandran, S. Kurkcuoglu and E. Rojas, The Star Product on the Fuzzy Supersphere, JHEP 0207, 056 (2002) [arXiv:hep-th/0204170];

[Balandin, Golubeva, 1999] V.V. Balandin, N.I. Golubeva, Hamiltonian Methods for the Study of Polarized Proton Beam Dynamics in Accelerators and Storage Rings, [arXiv: //arxiv . org/abs/physics/9903032v1].

[Bargmann, Michel,Telegdi, 1959] V. Bargmann, L. Michel, V.L. Telegdi, Precession of the polarization of particles moving in a homogeneous electromagnetic field, Phys. Rev. Lett., 2(10) (1959) 435.

[Barrow, Shaw, 2011] John D. Barrow, Douglas J. Shaw, The Value of the Cosmological Constant, General Relativity and Gravitation 43, 2555-2560 (2011), arXiv:1105.3105.

[Baskaran,2011] Baskaran, G. (2011). "Possibility of Skyrmion Superconductivity in Doped Antiferromagnet $\mathrm{K}_{2} \mathrm{Fe}_{4} \mathrm{Se}_{5} "$. arXiv: 1108.3562

[Benenti, Casati, Strini, 2004 ] G. Benenti, G. Casati, G. Strini, Principles of quantum computation and information, Vol. I: Basic concepts, World Scientific, Singapore 2004; Vol. II: Basic tools and special topics World Scientific, Singapore 2007.

[Beringer et al, 2012] J. Beringer et al. (Particle Data Group) Review of Particle Physics, Phys. Rev. D 86 (2012) 010001 [1528 pages]

[Berry, Keating, 1997] M. Berry, Speculations on the Riemann operator, in proceedings of Symposium on Supersymmetry and Trace formulae, Cambridge 1997.

[Bjorken,1966] J. D. Bjorken, Phys. Rev. 148, 1467 (1966).

[Berezin, 1987] F.A. Berezin, Introduction to Superanalysis, Reidel, Dordrecht 1987.

[Berezin, Marinov, 1977] F.A. Berezin, M.S. Marinov, Ann. Phys. (N.Y.) 104 (1977) 336.

[Beringer et al, 2012] J. Beringer et al. (Particle Data Group), PR D86, 010001 (2012)

[Blondel et al. 2013] A. Blondel et al., arXiv:1302.3318 [physics.acc-ph]; R. Belusevic and T. Higo, arXiv:1208.4956 [physics.acc-ph].

[Bodmer, 1971] A. Bodmer "Collapsed Nuclei" Phys. Rev. D4, 1601 (1971)

[Bogoliubov, Shirkov, 1959] N.N. Bogoliubov and D.V. Shirkov, Introduction to the Theory of Quantized Fields, New York 1959.

[Bogolubsky et al, 2009] I.L. Bogolubsky, E.M. Ilgenfritz, M. Müller-Preussker, A. Sternbeck, Phys.Lett.B 676 (2009) 69.

[Brandenberger,2004] R. H. Brandenberger, Lect. Notes Phys. 646:127-167 (2004), arXiv:hep-th/0306071.

[Brekke, Freund, 1993] L. Brekke, P.G.O. Freund, Phys. Rep. 233 (1993) 1.

[Brodsky, Farrar,1973] S. Brodsky, G. Farrar, Phys. Rev. Lett. 311153 (1973).

[Brodsky, de Tèramond, Deur, 2010] Stanley J. Brodsky, Guy F. de Tèramond, Alexandre Deur, Nonperturbative QCD Coupling and its $\beta$ function from Light-Front Holography, PRD 81,096010 (2010), arXiv:1002.3948

[Bronshtein, 1933] M. Bronshtein, K voprosu o vozmozhnoy teorii mira kak tselogo (On a possible theory of the world as a whole), in Uspekhi Astronomicheskikh Nauk, vypusk III (1933) pp. 3-30

[Brown and Rho, 1996] G. E. Brown and M. Rho, Phys. Rept. 269 (1996) 333 [hep-ph/9504250].

[Burkert,2001] V. D. Burkert, Phys. Rev. D 63, 097904 (2001).

[Buttin, 1996] C. Buttin, C.R. Acad. Sci. Paris. 269 (1969) 87. 
[Calcagni, 2009] G. Calcagni, Fractal universe and quantum gravity, Phys. Rev. Lett. 104 (2010) 251301 [arXiv:0912.3142].

[Carlip, 2009] S. Carlip, Spontaneous dimensional reduction in short-distance quantum gravity?, AIP Conf. Proc. 1196 (2009) 72 [arXiv:0909.3329].

[Castro Neto et al, 2009] A. H. Castro Neto, F. Guinea, N. M. R. Peres, K. S. Novoselov and A. K. Geim, The electronic properties of graphene, Rev. Mod. Phys. 81, 109-162 (2009).

[Caswell, 1974] W. E. Caswell, Phys. Rev. Lett. 33 (1974) 244.

[Chatrchyan et al. CMS Collaboration, 2012] S. Chatrchyan et al. [CMS Collaboration], Phys. Lett. B 716, 30 (2012).

[Collins, 1984] J.C. Collins, Renormalization, Cambrige Univ. Press, London 1984.

[Cox, Little, O’Shea 1998 ] David Cox, John Little, Donal O’Shea, Ideals, Varieties, and Algorithms, An Introduction to Computational Algebraic Geometry and Commutative Algebra, Springer 1998.

[Darbaidze et al, 1978] Ya.Z. Darbaidze, N.V. Makhaldiani, A.N. Sisakian, L.A. Slepchenko, TMF 34 (1978) 303.

[Davis, 1977] M. Davis, Applied nonstandard analysis, New York 1977.

[Diakonov, 2003] D. Diakonov, Prog. Par. Nucl. Phys. 51 (2003) 173.

[Di Giacomo, Paffuti, Rossi, 1992] A. Di Giacomo, G. Paffuti, P. Rossi, Selected Problems in Theoretical Physics [With Solutions], World Scientific 1992.

[Dirac, 1938] P.A.M. Dirac, Proc. Roy, Soc. A167 (1938) 148.

[Dirac, 1950, 1964] P.A.M. Dirac. Generalized Hamiltonian dynamics. Can. J. Math., 2:129, 148, 1950. P.A.M. Dirac. Generalized Hamiltonian dynamics. Proc. Roy. Soc. A, 246:326-332, 1958

P.A.M. Dirac. Lectures on Quantum Mechanics. Belfer Graduate School Monograph Series 3. Yeshiva University, New York, 1964.

[Drell, Hearn, 1966] S. D. Drell, A. C. Hearn, Exact Sum Rule For Nucleon Magnetic Moments, Phys. Rev. Lett. 16 (1966) 908.

[DiVincenzo, Peres, 1997] D. P. DiVincenzo and A. Peres, Quantum Codewords Contradict Local Realism, Physical Review A 55, pp. 4089-4092 (1997).

[Djukanovic et al, 2004] D. Djukanovic, M. R. Schindler, J. Gegelia, G. Japaridze, S. Scherer, Phys. Rev. Lett. 93 (2004) 122002.

[Duff, Okun, Veneziano, 2001] M. Duff, L. Okun and G. Veneziano, Trialogue on the number of fundamental constants, arXiv:physics/0110060.

[Duff, 2007] M. J. Duff, String triality, black hole entropy and CayleyŠs hyperdeterminant, Phys. Rev. D76 (2007) 025017.

R. Kallosh and A. Linde, Strings, black holes, and quantum information, Phys. Rev. D73 (2006) 104033. P. Levay, Stringy black holes and the geometry of entanglement, Phys. Rev. D t' 74 (2006) 024030.

[Dyson, Kleban, Susskind, 2002] Lisa Dyson, Matthew Kleban, Leonard Susskind, Disturbing Implications of a Cosmological Constant, JHEP 0210:011, 2002, arXiv:hep-th/0208013

[Egorian,Tarasov, 1979] E. Egorian and O. V. Tarasov, Theor. Math. Phys. 41 (1979) 863 [Teor. Mat. Fiz. 41 (1979) 26]

[Einstein, 1917] A. Einstein Sittzungsber.preuss. Akad. Wiss. 1 (1917) 142.

[Einstein, Podolsky, Rosen, 1935] A. Einstein, B. Podolsky, and N. Rosen, Can Quantum Mechanical Description of Physical Reality Be Considered Complete?, Physical Review 47, pp. 777-800 (1935).

[Elias, 2001] V. Elias, J. Phys. G. 27, 217 (2001). 
[Entangl1] M. Levin and X.-G. Wen, Detecting Topological Order in a Ground State Wave Function, Phys. Rev. Lett. 96, 110405 (2006) [arXiv:cond-mat/0510613];

A. Kitaev and J. Preskill, Topological entanglement entropy, Phys. Rev. Lett. 96, 110404 (2006) [arXiv:hep-th/0510092];

A. Hamma, R. Ionicioiu and P. Zanardi, Ground state entanglement and geometric entropy in the Kitaev's model, Phys. Lett. A 337, 22 (2005) [arXiv:quant-ph/0406202].

[Entang12] P. Calabrese and J. L. Cardy, Entanglement entropy and quantum field theory, J. Stat. Mech. 0406, P002 (2004) [arXiv:hep-th/0405152];

P. Calabrese and J. L. Cardy, Entanglement entropy and quantum field theory: A non-technical introduction, Int. J. Quant. Inf. 4, 429 (2006) [arXiv:quant-ph/0505193];

P. Calabrese and J. Cardy, Entanglement entropy and conformal field theory, J. Phys. A 42 (2009) 504005 [arXiv:0905.4013 [cond-mat.stat-mech]].

[Ernst, Schmit, 1976] W. Ernst, I. Schmitt, Nuovo Cim. A33 (1976) 195.

[Faddeev, Jackiw, 1988] L.D. Faddeev, R. Jackiw, Phys.Rev.Lett. 60 (1988) 1692.

[Faddeev, Takhtajan, 1990] L.D. Faddeev and L.A. Takhtajan, Hamiltonian methods in the theory of solitons, Springer, Berlin 1990.

[Farhi, Jaffe, 1984] E. Farhi and R. Jaffe, "Strange Matter", Phys. Rev. D30, 2379 (1984)

[Fermi, 1936] E. Fermi, Ricerca Scientifica 7 (1936) 13

[Ferrara, Porrati, Telegdi, 1992] S. Ferrara, M. Porrati, V.L. Telegdi, Phys. Rev. D, $g=2$ as the natural value of the tree level gyromagnetic ratio of elementary particles, 46 (1992) 3529.

[Feynman, 1955] Feynman, R. P. (1955). Application of quantum mechanics to liquid helium. Progress in Low Temperature Physics 1: 17Û53.

[Fialkovsky, Vassilevich, 2011] I. V. Fialkovsky, D. V. Vassilevich, Quantum Field Theory in Graphene, arXiv:1111.3017

[Fischer,Gies, 2004] C. S. Fischer and H. Gies, JHEP 0410 (2004) 048.

[Flambaum, Khriplovich, Sushkov, 1986] V. Flambaum, I. Khriplovich and O. Sushkov, Nucl.Phys. A449, p. 750 (1986).

[Friedan,1980] D.H. Friedan, Nonlinear models in two + epsilon dimensions, Phys. Rev. Lett. 45 (1980) 1057; Nonlinear models in two + epsilon dimensions, Ann. Phys. 163 (1985) 318.

[Fukuda,Žumer,2011] Fukuda, J.-I.; Žumer, S. (2011). "Quasi-two-dimensional Skyrmion lattices in a chiral nematic liquid crystal". Nature Communications 2: 246.

[Gamov, Ivanenko, Landau, 1928] G. Gamov, D. Ivanenko, L. Landau, Mirovye postoyannye i predel'ny perekhod [World constants and limiting transition], Zhurnal Russkogo Fiz.-Chim. Obschestva, chast' Fiz. 60 (1928) 13-17 (in Russian). Reprinted in Yad. Fiz. 65 (2002) No.7 [Physics of Atom. Nucl. 65 (2002) No.7].

[Gasper, Rahman, 1990] G. Gasper, M. Rahman, Basic Hypergeometric Series, Cambridge Univ. Press, Cambridge 1990.

[Gelfand et al, 1966] I.M. Gel'fand, M.I. Graev and I.I. Piatetskii-Shapiro, Representation Theory and Automorphic Functions, Saunders, London 1966.

[Gelfand et al, 1989] I.M. Gelfand, M.M. Kapranov, A.V. Zelevinsky, Funck. Anal. i Priloz. 23 (1989) 94;

[Gelfand et al, 1990] I.M. Gelfand, M.M. Kapranov, A.V. Zelevinsky, Adv. Math. 84 (1990) 255;

[Gelfand et al, 1992] I.M. GelŠfand, M.I. Graev, V.S. Retakh, Russian Math. Surveys 47 (1992) 1.

[Gerasimov, 1965] S.B. Gerasimov, A Sum Rule for Magnetic Moments and Damping of the Nucleon Magnetic Moment in Nuclei, J.Nucl.Phys.(USSR) 2 (1965) 598. 
[Gibbons, Hawking, 1977] Gibbons, G.W. and Hawking, S.W. (1977) 'Cosmological event horizons, thermodynamics, and particle creation' Physical Review D 15, 2738-2751

[Ginzburg, 1979] V. L. Ginzburg, Theoretical Physics and Astrophysics, Pergamon, New York 1979.

[Goldberger, Treiman, 1958] M.L. Goldberger, S.B. Treiman, Phys. Rev. 1101178 (1958).

[Gol'fand, Likhtman, 1971] Y.A. Gol'fand and E.P. Likhtman, JETP Lett., 13, 323, 1971.

[Gonalves et al, 2014] Vasco Gonalves, Joo Penedones, Emilio Trevisani, Factorization of Mellin amplitudes, arXiv:1410.4185v1 [hep-th] 15 Oct 2014.

[Green,Schwarz,Witten,1987] M.B. Green, J.H. Schwarz and E. Witten. Super String Theory. Vols 1,2. Cambridge University Press 1987.

[Greenberger, Horne, Zeilinger, 1989] D. M. Greenberger, M. Horne, and A. Zeilinger, Going beyond Bell's Theorem in Bell's Theorem, Quantum Theory, and Conceptions of the Universe, edited by M. Kafatos, pp. 73-76 (Kluwer, Dordrecht, 1989).

[Griffiths and Harris, 1994] Griffiths and Harris, Principles of Algebraic Geometry, [Wiley Classics Library Edition Published 1994]

[Gross, Wilczek, 1973] D.J. Gross, F. Wilczek, Phys. Rev. Lett. 301343 (1973).

[Grosse,Klimcik,Presnajde,1997] H. Grosse, C. Klimcik, P. Presnajder, Field Theory on a Supersymmetric Lattice, Commun.Math. Phys., 185 (1997) 155-175 and hep-th/9507074;H. Grosse, C. Klimcik, P. Presnajder, N=2 Superalgebra and Non-Commutative Geometry,hep-th/9603071;H. Grosse, G. Reiter, The Fuzzy Supersphere, J. Geom. and Phys., 28 (1998) 349-383 and math-ph/9804013.

[Gubser,Klebanov,Polyakov, 1998] S. S. Gubser, I. R. Klebanov and A. M. Polyakov, Phys. Lett. B 428, 105 (1998) [arXiv:hep-th/9802109];

[Gustavsson, 2007] A. Gustavsson, Algebraic structures on parallel M2-branes, Nucl. Phys. B.811 (2009) 66; [arXiv:0709.1260 [hep-th] ].

[Gutin, Pannen, 2002] G. Gutin, A.P. Punnen (Eds), The Traveling Salesman Problem and Its Variations, Combinatorial Optimization Series, Kluwer, Boston 2002.

[Hamilton,1982] R. Hamilton, Three-manifolds with positive Ricci curvature, J. Diff. Geom. 17 (1982) 255.

[Heinze et al,2011] Heinze, Stefan; Von Bergmann, Kirsten; Menzel, Matthias; Brede, Jens; Kubetzka, André; Wiesendanger, Roland; Bihlmayer, Gustav; Blügel, Stefan (2011). "Spontaneous atomic-scale magnetic skyrmion lattice in two dimensions". Nature Physics 7 (9): 713 Ú718.

[Heisenberg 1966] W. Heisenberg, Introduction to the Unified field Theory of Elementary particles, Interscience Publishers, London 1966.

['t Hooft, 1971] G. ’t Hooft, Nucl. Phys. B 33 (1971) 173; Nucl. Phys. B 35 (1971) 167.

['t Hooft, 1972 2] G. 't Hooft, Nucl.Phys. B 61 (1973) 455.

G. 't Hooft, Erice Lectures 1977 (unpublished).

['t Hooft, 1993] G. 't Hooft, Dimensional reduction in quantum gravity, in Salamfestschrift, A. Ali, J. Ellis, and S. Randjbar-Daemi (eds.), World Scientific, Singapore (1993) [gr-qc/9310026].

['t Hooft, 1972] G. 't Hooft, report at the Marseille Conference on Yang-Mills Fields, 1972.

['t Hooft, 1973] G. 't Hooft, Nucl.Phys. B 61 (1973) 455.

[Howe,Stelle,Townsend, 1983] P. Howe, K. Stelle and P. Townsend, Nucl. Phys. B214, 519 (1983).

[Howe,Stelle,West, 1983] P. Howe, K.S. Stelle and P. West, Phys. Lett. 124B, 55 (1983).

[Ernst-Michael Ilgenfritz, Axel Maas, 2012] Ernst-Michael Ilgenfritz, Axel Maas, Topological aspects of G2 Yang-Mills theory, arXiv:1210.5963. 
[Izergin, Korepin, 1982] A. Izergin and V. Korepin, Letter in Mathematical Physics vol 6, page 283, 1982.

[Jack,Jones,North, 1997] I. Jack, D.R.T. Jones and C.G. North, Nucl. Phys. B486, 479(1997).

[Jack,Jones,Pickering, 1998] I. Jack. D.R.T. Jones and A. Pickering, Phys. Lett. B435, 61 (1998).

[Jackson, 1999] J.D. Jackson, Classical Electrodynamics, 3rd ed. JohnWiley \& Sons, Inc. New York 1999.

[Ji,Osborne,2001] X. Ji, J.Osborne, J.Phys. G27 127 (2001).

[Jones,1974] D. R. T. Jones, Nucl. Phys. B 75 (1974) 531;

[Jons, 1983] D.R.T. Jones, Phys. Lett. 123B, 45 (1983).

[Kaku, 2000] M. Kaku, Strings, Conformal Fields, and M-Theory, Springer, New York 2000.

[Kataev, Larin, 2012] A. L. Kataev, S. A. Larin, Analytical five-loop expressions for the renormalization group QED $\beta$-function in different renormalization schemes, Pisma v ZhETF, vol.96, iss. 1, (2012), pp.64-67

[Kawarabayashi, Suzuki, 1966] K. Kawarabayashi, M. Suzuki, Phys. Rev. Lett. 16 (1966) 255

[Kazakov, 2004] D.I. Kazakov, Supersymmetric Generalization of the Standard Model of Fundamental Interactions, Textbook, JINR Dubna 2004.

[Kazakov, Shirkov, 1980] D.I. Kazakov, D.V. Shirkov, Fortschr. d. Phys. 28447 (1980).

[Kazakov et al, 1974] D.I. Kazakov, L.R. Lomidze, N.V. Makhaldiani, A.A. Vladimirov, Ultraviolet Asymptotics in Renormalizable Scalar Theories, JINR Communications, E2-8085, Dubna 1974.

[Khoze, 2013] Valentin V. Khoze, Inflation and Dark Matter in the Higgs Portal of Classically Scale Invariant Standard Model, arXiv:1308.6338v1 [hep-ph] 29 Aug 2013,

[Khriplovich, 1969] I. B. Khriplovich, Yad. Fiz. 10 (1969) 409 [Sov. J. Nucl. Phys. 10 (1970) 235]

[Kiselev et al,2011] Kiselev, N. S.; Bogdanov, A. N.; Schäfer, R.; Röler, U. K. (2011). "Chiral skyrmions in thin magnetic films: New objects for magnetic storage technologies?". Journal of Physics D: Applied Physics 44 (39): 392001. arXiv:1102.2726

[Kitaev, Shen, Vyalyi, 2002 ] A.Yu. Kitaev, A. Shen, M.N. Vyalyi, Classical and Quantum Computation, American Mathematical Society, 2002.

[Kitamoto, 2014] Takuya Kitamoto, On the Computation of the Determinant of a Generalized Vandermonde Matrix, in Computer Algebra, in Proceedings of "Scientific Computing, 16th International Workshop, CASC 2014" Warsaw, Poland, September 8-12, 2014, Vladimir P. Gerdt Wolfram Koepf Werner M. Seiler Evgenii V. Vorozhtsov (Eds.), Lecture Notes in Computer Science 8660, p.p. 242-255.

[Koba et al, 1972] Z. Koba, H.B. Nielsen, P. Olesen, Nucl. Phys. B40 (1972) 317.

[Koh,Rajpoo, 1983 ] I.G. Koh and S. Rajpoot, Phys. Lett. 135B, 397 (1983).

[Kulish et al, 1981-87] Kulish, P.P. and Reshetikhin, N.Yu., Zap. Sem. LOMI 101 (1981) 101 [J. Soviet. Math. 23 (1983) 2435].

Sklyanin, E.K., Funkt. Anal. Pril. 16 (1982) 27 [Funct. Anal. Appl. 16 (1982) 262].

Drinfel'd, V.G., DAN SSSR 283 (1985) 1060 [Soviet Math. Dokl. 32 (1985) 254] ; in Proc. Int. Congr. of

Mathematics, ed. A.M. Gleason, AMS : Providence (1986).

Jimbo, M., Lett. Math. Phys. 10 (1985) 63 ; 11 (1986) 247 ; Commun. Math. Phys. 102 (1986) 537.

Woronowicz, S.L., Publ. RIMS-Kyoto 23 (1987) 117 ; Commun. Math. Phys. 111 (1987) 613.

[Kuster et al, 2008] M. Kuster, G. Raffelt and B. Beltran (eds), Lecture Notes in Physics 741 (2008).

[Laughlin, 1981] Laughlin, R. (1981). "Quantized Hall conductivity in two dimensions". Physical Review B 23 (10): 5632 Ű5633.

[Larin,Vermaseren,1993] S. A. Larin and J. A. M. Vermaseren, Phys. Lett. B 303 (1993) 334. 
[Lerche,von Smekal, 2002] C. Lerche and L. von Smekal, Phys. Rev. D 65 (2002) 125006.

[Lomidze, 1994] I. Lomidze, On Some Generalizations of the Vandermonde Matrix and their Relations with the Eiler Beta-function, Georgian Math. Journal 1 (1994) 405.

[Lomidze, Makhaldiani, 2012] I. R. Lomidze and N. V. Makhaldiani, SOME PROPERTIES OF THE GENERALIZED EULER BETA FUNCTION, Memoirs on Differential Equations and Mathematical Physics 56 (2012)

[Lucchesi,Piguet,Sibold,1988] C. Lucchesi, O. Piguet and K. Sibold, Helv. Phys. Acta 61 (1988) 321

[Lucchesi,Zoupanos,1997] C. Lucchesi and G. Zoupanos, Fortschr. Phys. 45 (1997) 129, hep-ph/9604216

[Lushikov, 1969] V.I. Lushikov et al., Sov. Phys. JETP Lett. 9 (1969) 23

[Makhaldiani, 1980] N.V. Makhaldiani, Approximate methods of the field theory and their applications in physics of high energy, condensed matter, plasma and hydrodynamics, Dubna 1980.

[Makhaldiani, 1986] N.M. Makhaldiani, Computational Quantum Field Theory, JINR Communication, P2-86-849, Dubna 1986.

[Makhaldiani, 1987] N.V. Makhaldiani, A New Approach to the Problem of Space Compactification, JINR Communications, P2-87-306, Dubna 1987.

[Makhaldiani, 1988] N.V. Makhaldiani, Number Fields Dynamics and the Compactification of Space Problem in the Unified Theories of Fields and Strings, JINR Communications, P2-88-916, Dubna 1988.

[Makhaldiani, 1997,2] N. Makhaldiani, The System of Three Vortexes of Two-Dimensional Ideal Hydrodynamics as a New Example of the (Integrable) Nambu-Poisson Mechanics, JINR Communications E2-97-407, Dubna 1997; [arXiv: solv-int/9804002].

[Makhaldiani, 1999] N. Makhaldiani, The Algebras of the Integrals of Motion and Modified Bochner-Killing-Yano Structures of the Point particle Dynamics, JINR Communications E2-99-337, Dubna 1999.

[Makhaldiani, 2000] N. Makhaldiani, New Hamiltonization of the Schrödinger Equation by Corresponding Nonlinear Equation for the Potential, JINR Communications, E2-2000-179, Dubna 2000.

[Makhaldiani, 2001] N. Makhaldiani, How to Solve the Classical Problems on Quantum Computers, JINR Communications, E2-2001-137, Dubna 2001.

[Makhaldiani, 2002] N. Makhaldiani, Classical and Quantum Problems for Quanputers, [arXiv:quant-ph/0210184].

[Makhaldiani, 2003] N. Makhaldiani, Adelic Universe and Cosmological Constant, JINR Communications, E2-2003-215 Dubna 2003, [arXiv: hep-th/0312291].

[Makhaldiani, 2007] N. Makhaldiani, Nambu-Poisson dynamics of superintegrable systems, Atomic Nuclei, 70 (2007) 564.

[Makhaldiani, 2007.2] N. Makhaldiani, Theory of Quanputers, Sovremennaia Matematica i ee Prilozhenia, 44 (2007) 113; Journal of Mathematical Sciences, 153 (2008) 159.

[Makhaldiani, 2008] N.V. Makhaldiani, Renormdynamics and Scaling Functions, in Proc. of the XIX International Baldin Seminar on High Energy Physics Problems eds. A.N.Sissakian, V.V.Burov, A.I.Malakhov, S.G.Bondartenko, E.B.Plekhanov, Vol.II, p. 175, Dubna 2008.

[Makhaldiani, 2010] N.V. Makhaldiani, Renormdynamics, multiparticle production, negative binomial distribution and Riemann zeta function, [arXiv:1012.5939v1 [math-ph] ] 24 Dec 2010.

[Makhaldiani, 2011] N. Makhaldiani, Fractal Calculus (H) and some Applications, Physics of Particles and Nuclei Letters, 8325 (2011). 
[Makhaldiani, 2011.2] N. Makhaldiani, Regular method of construction of the reversible dynamical systems and their linear extensions - Quanputers, Atomic Nuclei, 74 (2011) 1040.

[Makhaldiani, 2012] Nugzar Makhaldiani, Nambu-Poisson Dynamics with Some Applications, Physics of Particles and Nuclei, 43 (2012) 703.

[Makhaldiani, 2012.2] Nugzar Makhaldiani, Renormdynamics, coupling constant unification and universal properties of the multiparticle production, XXI International Baldin Seminar on High Energy Physics Problems, September 10-15, 2012, PoS(Baldin ISHEPP XXI)068.

[Makhaldiani, 2013] N. V. Makhaldiani, Renormdynamics, Multiparticle Production, Negative Binomial Distribution, and Riemann Zeta Function, Physics of Atomic Nuclei, 761169 (2013).

[Makhaldiani, Voskresenskaya, 1997] N. Makhaldiani, O. Voskresenskaya, On the correspondence between the dynamics with odd and even brackets and generalized Numbu's mechanics, JINR Communications, E2-97-418, Dubna 1997.

[Maldacena, 1999] J. M. Maldacena, Adv. Theor. Math. Phys. 2, 231 (1998) [Int. J. Theor. Phys. 38, 1113 (1999)] [arXiv:hep-th/9711200].

[Mandelstam, 1983] S. Mandelstam, Nucl. Phys. B213, 149 (1983).

[Matveev, Muradyan,Tavkhelidze,1973] V. Matveev, R. Muradyan, A. Tavkhelidze, Lett. Nuovo Cimento 7719 (1973).

[Matveev et al, 1976] V.A. Matveev, A.N. Sisakian, L.A. Slepchenko, Nucl. Phys. 23 (1976) 432.

[Meleshko,Konstantinov, 1993] A.V. Meleshko, N.N. Konstantinov, Dynamics of vortex systems, Naukova Dumka, Kiev 1993.

[Miller, 1977] W. Miller, Jr. Symmetry and Separation of Variables, Addison-Wesley PC, London 1977.

[Misner,Thorne,Wheeler,2000] C.W. Misner, K.S. Thorne, J.A. Wheeler. Gravitation. W.H.Freeman and Company, twenty third printing 2000 .

[Morgan,2007] J.W. Morgan and G. Tian, Ricci Flow and the Poincaré Conjecture, Clay Mathematics Monographs, Amer. Math. Soc., Cambridge, 2007 [math.DG/0607607].

[MSSMHiggs,1991-1993] J. Ellis, G. Ridolfi and F. Zwirner, Phys. Lett. 262B (1991) 477; R. Barbieri and M. Frigeni, Phys. Lett. 258B (1991) 395; A. Brignole et al., Phys. Lett. 271B (1991) 123; M. Carena, K. Sasaki and C.E.M. Wagner, Nucl. Phys. 381B (1992) 66; H.E. Haber, R. Hempfling, Phys. Rev. D48 (1993) 4280; P.H. Chankowski, S. Pokorski and J. Rosiek, Phys. Lett. 281B (1992) 100

[Mumford, 1983] D. Mumford, Tata Lectures on Theta, Birkhäuser 1983.

[Nambu, 1973] Y. Nambu, Phys.Rev. D 7 (1973) 2405.

[Neveu,Schwarz,1971 ] A. Neveu and J.H. Schwarz, Nucl. Phys., B31, 86, 1971.

[Nielsen, Chuang, 2000 ] M.A. Nielsen, I.L. Chuang, Quantum computation and quantum information, Cambridge University Press, Cambridge 2000.

[Nilles, 1984] H.P. Nilles, Phys. Rept. 110 (1984) 1; H.E. Haber and G.L. Kane, Phys. Rept. 117 (1985) 75; R. Barbieri, Riv. Nuovo Cim. 11 (1988) 1.

[Novikov et al 1983] V. Novikov, V. Shifman, A. Vainshtein and V. Zakharov, Nucl. Phys. B229, 381 (1983).

[Novikov et al, 1984] S. P. Novikov, S. V.Manakov, L. B. Pitaevskii, and V. E. Zakharov, Theory of solitons. The inverse scattering method, Plenum Press, New York, 1984.

[Oehme, Zimmermann, 1985] R. Oehme and W. Zimmermann, Commun. Math. Phys. 97 (1985) 569.

[Okun, 1991] L. Okun, The fundamental constants of physics, Sov. Phys. Usp. 34 (1991) 818.

[Pauli, 1925] W. Pauli, Z. Physik 31765 (1925). 
[Pauli, 1940] W. Pauli, Phys. Rev. 58716 (1940).

[Pati,Salam,1974] J. C. Pati and A. Salam, Physical Review D10, 275 (1974).

[Pawlowski et al, 2004] J. M. Pawlowski, D. F. Litim, S. Nedelko and L. von Smekal, Phys. Rev. Lett. 93 (2004) 152002.

[Perelman,2002-2003] G. Perelman, The entropy formula for the Ricci flow and its geometric applications [math.DG/0211159]; Ricci flow with surgery on three-manifolds [math.DG/0303109]; Finite extinction time for the solutions to the Ricci flow on certain three-manifolds [math.DG/0307245].

[Perlmutter et al, 1999] S. Perlmutter et al., Ap.J. 517, 565 (1999).

[Peskin, 2012] M. E. Peskin, arXiv:1207.2516 [hep-ph].

[Piguet,Sibold,1986] O. Piguet and K. Sibold, Int. J. Mod. Phys. A1 (1986) 913;

[Planck, 1899] M. Planck, Über irrevesible Strahlungsvorgänge, S.-B. Preuss Akad. Wiss. (1899) 440-480; Ann. d. Phys. 1 (1900) 69 reprinted in Max Planck, Physikalische Abhandlungen und Vorträge, Band I. Friedr. Vieweg. 1958, pp. 560-600, pp. 614-667.

[Politzer, 1973] H.D. Politzer, Phys. Rev. Lett. 301346 (1973).

[Pontryagin, 1983] L.S. Pontriagin et al., Mathematical Theory of Optimal Processes, Nauka, Moscow 1983.

[Ramond, 1971] P. Ramond, Phys. Rev., D3, 2415, 1971.

[Rentmeester et al, 1999] M.C.M. Rentmeester, R.G.E. Timmermans, J.L. Friar, J.J. de Swart, Phys. Rev. Lett. 82 4992 (1999).

[Riazuddin, Fayyazuddin, 1966] Riazuddin, Fayyazuddin, Phys. Rev. 147 (1966) 1071.

[Riess et al, 1998] A.G. Riess et al., Ap.J. 116, 1009 (1998);

[van Ritbergen,Vermaseren,Larin,1997] T. van Ritbergen, J. A. M. Vermaseren and S. A. Larin, Phys. Lett. B 400 (1997)379.

[Roberts, Marciano, 2010] B. L. Roberts and W. J. Marciano (eds.), Lepton Dipole Moments, Advanced Series on Directions in High Energy Physics, Vol. 20 (World Scientific, 2010).

[Romming et al,2013] Romming, N.; Hanneken, C.; Menzel, M.; Bickel, J. E.; Wolter, B.; Von Bergmann, K.; Kubetzka, A.; Wiesendanger, R. (2013). "Writing and Deleting Single Magnetic Skyrmions". Science 341 (6146): 636Ü9

[Sakharov, 1967] A. Sakharov, Pisma Zh.Eksp.Teor.Fiz. 5, 32 (1967), Reprinted in Sov. Phys. Usp. 34 (1991) 392-393 [Usp. Fiz. Nauk 161 (1991) No. 5 61-64].

[Sakurai, 1969] J.J. Sakurai, Currents and mesons, The University of Chicago Press, Chicago 1969.

[Samarskii, Gulin, 1989] A. Samarskii, A. Gulin, Numerical Methods, Nauka, Moscow 1989.

[Schmidhuber,Tseytlin,1994] C. Schmidhuber and A.A. Tseytlin, On string cosmology and the RG flow in 2-d field theory, Nucl. Phys. B426 (1994) 187 [arXiv:hep-th/9404180].

[Schlienz, Mahler, 1995] J. Schlienz and G. Mahler, Description of Entanglement, Physical Review A 52, pp. 4396-4404 (1995).

[Schlienz, Mahler, 1996] J. Schlienz and G. Mahler, The maximal entangled threeparticle state is unique, Physics Letters A 224, pp. 39-44 (1996).

[Schwinger, 1966] J. Schwinger, Magnetic Charge and Quantum Field Theory, Phys. Rev. 144 (1966) 1087.

[Schwinger, 1983] Schwinger, J. Electromagnetic mass revisited. Found. Phys. 13, 373 (1983). 
[Seiler, Tucker, 2014] Werner M. Seiler, Robin W. Tucker, Involution and Constrained Dynamics I: The Dirac Approach, in Proceedings of "Scientific Computing, 16th International Workshop, CASC 2014" Warsaw, Poland, September 8-12, 2014, Vladimir P. Gerdt Wolfram Koepf Werner M. Seiler Evgenii V. Vorozhtsov (Eds.), Lecture Notes in Computer Science 8660, p.p. 242-255.

[Sinai, 1993] Ya.G. Sinai, Topics in Ergodic Theory, Princeton University Press, Princeton NJ 1993.

[Steyerl, 1969] A. Steyerl, Phys. Lett. B29 (1969) 33

[Stoney, 1881] G.J. Stoney, The philosophical magazine and journal of science, 11 (1881) 381.

[Tarasov,Vladimirov,Zharkov,1980] O. V. Tarasov, A. A. Vladimirov and A. Y. Zharkov, Phys. Lett. B 93 (1980) 429.

[Taylor, Veneziano, 1988] T.R. Taylor, and G. Veneziano, Dilaton Couplings at Large Distance, Phys. Lett. B 213, 450 (1988).

[Tegmark, 2004] Tegmark, Max; et al. (2004). Cosmological parameters from SDSS and WMAP. Physical Review D 69 (103501): 103501. arXiv:astro-ph/0310723.

[Titchmarsh, 1986] E.C. Titchmarsh, The Theory of the Riemann zeta-function, Clarendon Press, Oxford 1986.

[Toffoli, Margolus, 1987] Norman Margolus, Tommaso Toffoli, Cellular Automaton Machines, MIT Press, Cambridge 1987.

[Tokarev, Zborovsky, 2007] M.V. Tokarev, I. Zborovský, Z-Scaling in the Proton-Proton Collisions at RHIC, in Investigations of Properties of Nuclear Matter at High Temperature and Densities, Editid by A.N. Sisakian, F.A. Soifer, Dubna 2007

[Tomas, 1927] L.H. Thomas, Philos. Mag. 3 (1927) 1.

[Treiman, Witten, Jackiw, Zumino, 1985] S. B. Treiman, E. Witten, R. Jackiw, B. Zumino, Current Algebra and Anomalies, World Scientific, Singapore 1985).

[Tzalenchuk, 2010] Alexander Tzalenchuk et al, Quantum Resistance Standard Based on Epitaxial Graphene, Nature Nanotechnology 5, 186 - 189 (2010).

[Vanyashin, Terentyev, 1965] V. S. Vanyashin and M. T. Terentyev, Sov. Phys. JETP 21 (1965) 375.

[Veneziano, 1986] G. Veneziano, A stringy nature needs just two constants, Europhys. Lett. 2 (1986) 199.

[Veneziano, 1998] G. Veneziano, A Simple/Short Introduction to Pre-Big-Bang Physics/Cosmology, arXiv:hep-th/9802057

[Vilenkin, 1995] A. Vilenkin, Phys. Rev. Lett. 74, 864 (1995).

[Vladimirov, 1988] V.S. Vladimirov, Russian Math. Surveys 43 (1988) 19.

[Voloshin, Ter-Martyrosian, 1984] M.B. Voloshin, K.A. Ter-Martyrosian, Gauge Theory of Elementary Particles, Atomizdat, Moscow 1984.

[Watkins] M. Watkins at http://secamlocal.ex.ac.uk/ mwatkins/zeta/physics.htm.

[Weinberg, 1968] S. Weinberg, Phys. Rev. 166 (1968) 1568

[Weinberg, 1972] S. Weinberg, Gravitation and Cosmology, New York 1972.

[Weinberg, 1987] S. Weinberg (1987). "Anthropic Bound on the Cosmological Constant". Phys. Rev. Lett. 59 (22): $2607-2610$

[Weinberg, 1989] S. Weinberg, Rev. Mod. Phys. 49 (1989) 1.

[Weinberg, 1995] S. Weinberg, The Quantum Theory of Fields, Volum I - Foundations, Cambridge Univ. Press 1995.

[Weinberg, 1996] S. Weinberg, The Quantum Theory of Fields, Volum II - Modern Applications, Cambridge Univ. Press 1996. 
[Weinberg, 2000] S. Weinberg, The Quantum Theory of Fields, Volum III - Supersymmetry, Cambridge Univ. Press 2000 .

[Weinberg, 1972] S. Weinberg. Gravitation and Cosmology: Principles and Applications of the General Theory of Relativity. John Wiley and Sons, Inc. 1 edition, 1972;

[Weinberg, 1993] S. Weinberg, AIP Conf.Proc. 272, 346 (1993).

[Weisberger, 1966] W. I. Weisberger, Phys. Rev. 143 (1966) 1302.

[Wess,Zumino,1974] J. Wess and B. Zumino, Nucl. Phys., B70, 39, 1974

[Whittaker, 1927] E.T. Whittaker, A Treatise on the Analytical Dynamics, Cambridge 1927.

[Will, 2006] C. M. Will, The Confrontation between General Relativity and Experiment, Living Rev. Relativity 9, (2006), http://www.livingreviews.org/lrr-2006-3.

[Witten, 1998] E. Witten, Adv. Theor. Math. Phys. 2, 253 (1998) [arXiv:hep-th/9802150].

[Witten, 1984] E. Witten, "Cosmic Separation Of Phases" Phys. Rev. D30, 272 (1984)

[Witten, 1984,2] E. Witten, Some Properties of O(32) Superstrings, Phys. Lett. B 149, 351 (1984).

[Zeldovich, 1959] Ya.B. Zeldovich, Sov. Phys. JETP-9 (1959) 1389

[Zelmanov, 1967] A. Zelmanov, Kosmologia (Cosmology), in: Razvitie astronomii v SSSR [Development of astronomy in USSR], Nauka, Moscow, 1967, pp. 320-390, in particular p. 323 (in Russian).

[Zimmermann, 1980] W. Zimmermann, ŞThe Renormalization Group of the Model of A4-Coupling in the Abstract Approach of Quantum Field Theory $\breve{T}$, Commun. Math. Phys. 76 (1980) 39 Ü64.

[Zimmermann, 1985] W. Zimmermann, Commun. Math. Phys. 97 (1985) 211

[Zwanziger, 2002] D. Zwanziger, Phys. Rev. D 65 (2002) 094039. 